\title{
The integration of extrinsic and intrinsic neuromodulators in the olfactory system
}

\author{
Kristyn M. Lizbinski
}

West Virginia University, kmlizbinski@mix.wvu.edu

Follow this and additional works at: https://researchrepository.wvu.edu/etd

Part of the Systems Neuroscience Commons

\section{Recommended Citation}

Lizbinski, Kristyn M., "The integration of extrinsic and intrinsic neuromodulators in the olfactory system" (2018). Graduate Theses, Dissertations, and Problem Reports. 3753.

https://researchrepository.wvu.edu/etd/3753

This Dissertation is protected by copyright and/or related rights. It has been brought to you by the The Research Repository @ WVU with permission from the rights-holder(s). You are free to use this Dissertation in any way that is permitted by the copyright and related rights legislation that applies to your use. For other uses you must obtain permission from the rights-holder(s) directly, unless additional rights are indicated by a Creative Commons license in the record and/ or on the work itself. This Dissertation has been accepted for inclusion in WVU Graduate Theses, Dissertations, and Problem Reports collection by an authorized administrator of The Research Repository @ WVU.

For more information, please contact researchrepository@mail.wvu.edu. 
The integration of extrinsic and intrinsic neuromodulators in the olfactory system

\author{
Kristyn M Lizbinski \\ Dissertation submitted to the Eberly College of Arts and Sciences \\ at West Virginia University \\ in partial fulfillment of the requirements \\ for the degree of \\ Doctor of Philosophy \\ in \\ Biology
}

\begin{abstract}
Andrew M Dacks, Ph.D., Committee Chairperson
Kevin C Daly, Ph.D.

Gary Marsat, Ph.D.

Sarah M Farris, Ph.D.

Sergiy Yakovenko, Ph.D.

Department of Biology
\end{abstract}

Morgantown, West Virginia

November 2018

Keywords: neuromodulation, olfaction, serotonin, dopamine, GABA, neuropeptides

Copyright 2018 Lizbinski 


\section{Abstract \\ The integration of extrinsic and intrinsic neuromodulators in the olfactory system Kristyn M Lizbinski}

Neuromodulation is a ubiquitous feature of neural systems, allowing flexible, context specific control over network dynamics by adjusting the biophysical and synaptic properties of neurons. Neuromodulation was first described in invertebrate motor systems and early work established a basic dichotomy for neuromodulation as having either an intrinsic origin (i.e. neurons that participate in network coding) or an extrinsic origin (i.e. neurons from independent networks). Although this dichotomy has been thoroughly considered in motor systems, it has received far less attention in sensory systems. Furthermore, nervous systems are continually subject to a dynamic cocktail of both intrinsic and extrinsic modulators. However, the mechanisms by which single neurons integrate the influence of multiple modulators to alter network function are relatively unexplored. In this dissertation, I discuss the mechanisms by which intrinsic and extrinsic modulators are integrated by single neurons as well as network wide within the context of olfactory processing in the moth Manduca sexta. I begin by discussing the anatomical basis for the integration of two extrinsic modulators, serotonin and dopamine, on principal olfactory neurons. I then discuss the cell-class specific physiological effects of serotonin and dopamine and their distinct effects on olfactory processing. Finally, I discuss the organizing principles and heterogeneity of a diverse group of intrinsic modulatory local interneurons and their potential role in modulating olfactory network dynamics. The heterogeneous nature of neuromodulation is a recurring theme throughout this dissertation as the effects of both intrinsic and extrinsic modulation are generally non-uniform. 


\section{DEDICATION}

\section{To Mom:}

You never missed a single soccer game, concert, or academic achievement. Thank you for teaching me the importance of a good sing-along, the healing power of ice cream, and most importantly, your unconditional support and love.

\section{To Dad:}

My original adventure buddy. I love our daily after-work phone calls. Thank you for always believing me even when I didn't believe in myself. I cherish our mountainside chats, and pondering life over a nice glass of wine.

To Josh:

You're the smartest and most creative person I know. Thanks for being the best brother I could ask for.

To Tyler:

You're my person, my love, and my best friend. You make me better. Thank you for being my kind of weird, and for your continual love and support. 


\section{ACKNOWLEDGEMENTS}

I am the product of all the people who believed in me, taught me, sacrificed time, went before me, and supported me along this journey.

First, I would like to thank my advisor, Andrew Dacks. I am honored to be your first PhD student. You're an amazing scientist and an even better person. You taught me to see the forest through the trees. I have grown so much during my time in your lab thanks to your creative thinking, motivation, and encouragement. Thank you for everything. I could not ask for a better mentor.

To my family, I am incredibly lucky to have such a supportive and loving group of people cheering me on. Nanny, you always send me back to school with care packages filled with homemade food. You helped me have a little piece of home during stressful times. Grammy, this research is also in honor of you and all families affected by Alzheimer's.

Thank you to my past teachers. You instilled in me a passion for learning. Specifically, Marc Seid; you taught me that insects have beautiful brains and that being a creative scientist could be a career choice. Without my undergraduate experience in your lab, I wouldn't be where I am today.

To my wonderful friends who have provided countless hours of support, listened to my struggles, and were there during the ups and downs: Diana Anthony, Carina Hanks, Kristen Patchcoski, Olivia Annitti, Amanda Balasko, Kate Allen.

To the Dacks lab members, past and present, specifically Kaylynn Coates, Ayad Auda, and my personal favorite Tyler Sizemore. Thank you also to members of the Daly lab for rearing the moths and your help, specifically Philip Chapman.

Thank you to my committee for your diverse skillset, support, and great questions: Kevin Daly, Gary Marsat, Sarah Farris and Sergiy Yakovenko.

Thank you to the WVU Department of Biology. Specifically, Richard Thomas for your stellar role as chair of the department and your advocacy for grad students, and Mickey Howell for taking care of us all with food, support, and kindness.

I'd also like to thank my co-authors Aditya Kesari, Samuel Bradley and Jackie Metheny.

Finally, thank you to the moths. I literally could not have done this without you. 


\section{TABLE OF CONTENTS}

\section{Chapter 1}

Introduction

\section{Chapter 2}

The Anatomical Basis for Modulatory Integration of Serotonin and Dopamine in the Antennal Lobe

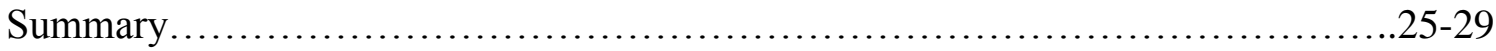

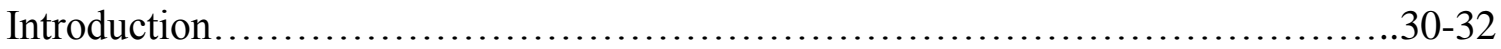

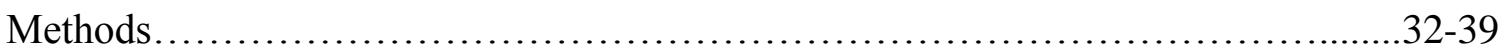

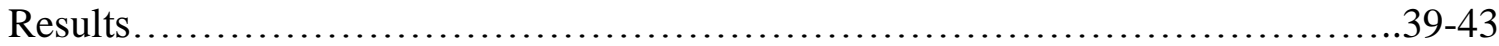

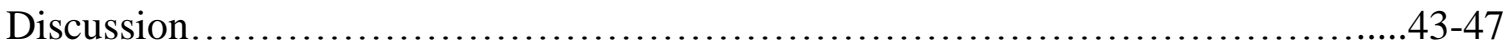

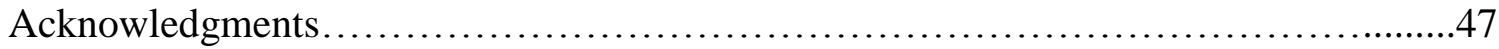

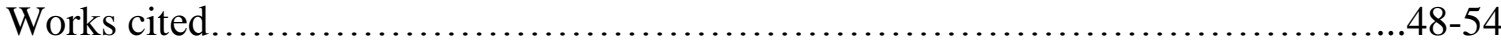

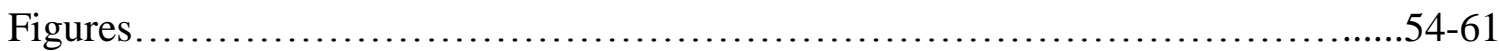

\section{Chapter 3}

Serotonin and Dopamine Modulate Olfactory Coding via Distinct Mechanisms

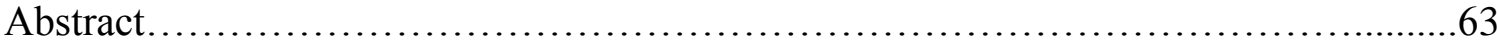

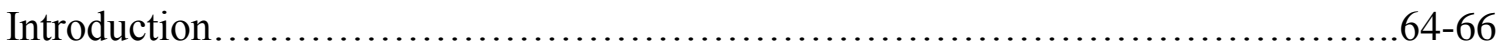

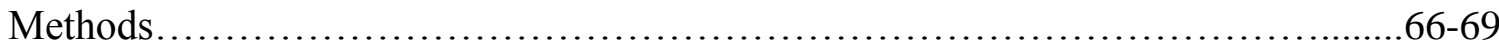

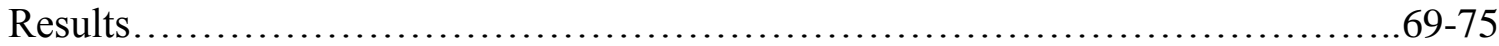

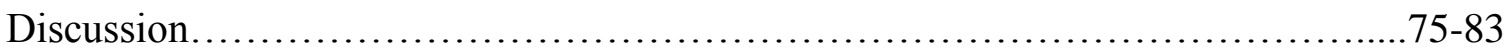

Acknowledgments ................................................................ 83

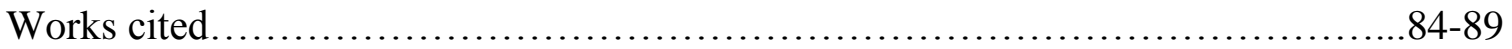

Figures........................................................... $90-96$

\section{Chapter 4}

Systematic Analysis of Transmitter Co-expression Reveals Organizing Principles of Olfactory Local Interneuron Heterogeneity

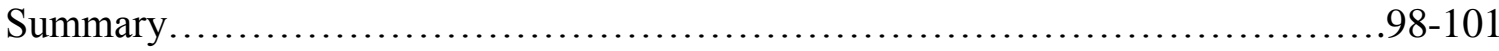

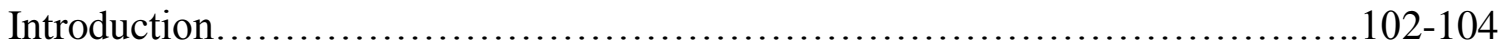

Methods.............................................................105-112

Results............................................................... 112-117

Discussion.............................................................. 117-123

Acknowledgments..................................................123-124

Works cited ............................................................ 124-130

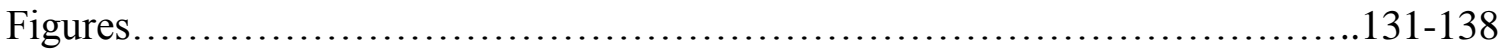

\section{Chapter 5}

Discussion and Future Directions...............................................139-155 
CHAPTER 1

Introduction 
(portions of this chapter are taken from my publication "Lizbinski, K. M., \& Dacks, A. M. (2018). Intrinsic and extrinsic neuromodulation of olfactory processing. Frontiers in cellular neuroscience, 11, 424.)

\section{Introduction:}

Neuromodulation adjusts the biophysical and synaptic properties of neurons, allowing fine control over neural network dynamics (Kupfermann, 1979; Kaczmarek and Levitan, 1987; Katz, 1999). Foundational work from invertebrate motor systems (Harris-Warrick and Marder, 1991; Katz, 1995; Katz and Frost 1995, 1996) identified two major categories of neuromodulation that modify network processing under different circumstances; intrinsic neuromodulation vs. extrinsic neuromodulation. Intrinsic neuromodulation is exerted by neurons that are within a neural network and participate in information processing undertaken by that network. The amount of intrinsic neuromodulation depends on past or ongoing network activity and provides a "history" of the network state. Extrinsic neuromodulation is exerted by neurons that originate in independent networks, such as centrifugal neurons or humoral factors, and therefore provide information based on the activity of other neural networks. The amount of extrinsic neuromodulation therefore depends on the activity of other networks, rather than the network that is being modulated. Thus, extrinsic neuromodulation provides a context about the broader state of the animal. The buccal ganglion of the sea slug Aplysia californica, which coordinates motor output to control biting movements, illustrates the influence of both intrinsic and extrinsic modulation within a single network (Morgan et al., 2000). The cerebral interneuron "CBI-2" initiates and directly participates in biting motor programs, making it an intrinsic element of the feeding central pattern generator (CPG). With each motor program, CBI-2 improves bite articulation via several neuromodulators (Morgan et al., 2000; Koh and Weiss, 2005, 2007; Friedman and Weiss, 2010; Dacks et al., 2012a). Biting motor programs can also be modulated based on prior exposure to food (Kupfermann, 1974) via the serotonergic metacerebral cells (MCC) which are external to the feeding CPG. The MCCs do not initiate biting motor programs, yet their activity decreases latency of motor program initiation (Kupfermann, 1979; Kupfermann and Weiss, 1982; Morgan et al., 2000) and lesioning the MCCs reduces the frequency of biting (Rosen et al., 1989). Finally, the MCCs (the extrinsic modulators) shorten feeding motor program latency by increasing CBI-2 (the intrinsic modulator) quantal content (Proekt and Weiss, 2003). This "metamodulation", i.e. modulation of 
neuromodulation, demonstrates how extrinsic modulators can target intrinsic modulatory components to exert contextual control over network activity (Katz, 1999).

While this conceptual dichotomy (intrinsic vs. extrinsic neuromodulation) has been extensively explored in motor systems, it has received less attention in sensory systems and the mechanisms by which single neurons integrate multiple modulators are underexplored. In this dissertation, I take advantage of the depth of groundwork on individual neuromodulators in insect nervous systems to determine the mechanisms by which intrinsic and extrinsic modulators are integrated within the first processing stage of the olfactory system of the hawkmoth, Manduca sexta. The insect antennal lobe (AL), analogous to the vertebrate olfactory bulb, is an excellent system in which to approach this problem due to the wealth of information on olfactory system neuron types, their physiology, morphology and transmitter content combined with the brain's relative numerical simplicity. I begin by discussing the anatomy of the olfactory system, and outlining the known mechanisms of two extrinsic modulators, serotonin and dopamine. I then discuss the contribution of local interneurons to intrinsic modulation of olfactory processing, specifically presynaptic gain control. The heterogeneous nature of neuromodulation is a recurring

theme throughout this dissertation as the effects of both intrinsic and extrinsic modulation are generally non-uniform.

\section{Anatomy of the olfactory system and sources of neuromodulation}

In the insect antennal lobe (AL), odorants bind to chemosensory proteins expressed by input neurons called olfactory receptor neurons (ORNs) (Figure 1). Individual ORNs typically express a single chemosensory receptor protein (Vosshall et al., 1999; Vosshall, 2000; Goldman et al., 2005; Joseph and Carlson, 2015) and all ORNs that express the same chemosensory receptor protein project into the same sub-structure in the AL called a glomerulus. Within a glomerulus, ORNs synapse upon output neurons called projection neurons (PNs). PNs then send olfactory information to higher order brain centers like the mushroom bodies (involved in learning/memory; reviewed in (Zars, 2000; Owald and Waddell, 2015) and the lateral horn (involved in odor valence; 
Gupta and Stopfer, 2011; Sachse and Beshel, 2016; Schultzhaus et al., 2017). Finally, a diverse population of local interneurons (LNs) (Seki and Kanzaki, 2008; Chou et al., 2010; Seki et al., 2010; Reisenman et al., 2011) refines the input/output relationship of ORNs and PNs. All three principal neuron types, ORNs, LNs, and PNs, are subject to both intrinsic and extrinsic sources of neuromodulation. LNs release GABA, dopamine (in some insects, DA) and a suite of neuropeptides (Homberg et al., 1990; Kirchhof et al., 1999; Berg et al., 2007; Utz et al., 2008; Carlsson et al., 2010; Chou et al., 2010; Siju et al., 2014; Fusca et al., 2015; Hamanaka et al., 2016; Tedjakumala et al., 2017), while the AL is innervated by centrifugal neurons releasing serotonin (5-HT), DA and octopamine (OCT) which act as extrinsic modulators (Kent et al., 1987; Rehder et al., 1987; Salecker and Distler, 1990; Ignell, 2001; Dacks et al., 2005, 2006, 2012a; Sinakevitch et al., 2005; Sinakevitch and Strausfeld, 2006).

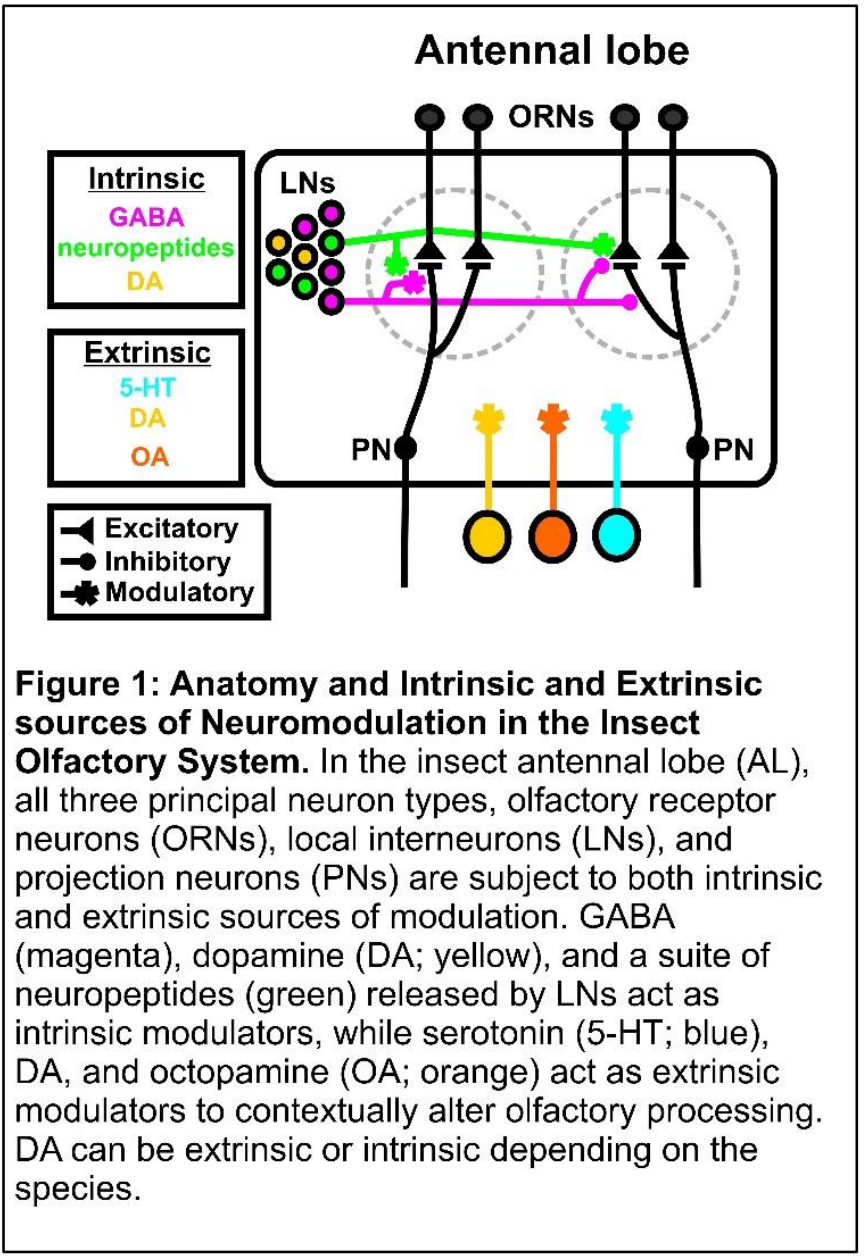

\section{Extrinsic Modulation of Olfactory Processing}

Animals must constantly adjust their sensory processing to meet the ongoing demands of a dynamic internal and external environment. Both insects and vertebrates heavily rely on their sense of smell to find mates, acquire food and avoid harmful threats in their environment. However, the relative importance of any given odor varies with current physiological demands. Extrinsic modulatory neurons from other networks can therefore adjust activity within the AL to provide the context of current internal demands of the individual animal. The olfactory system is subject to a number of extrinsic sources of neuromodulation including 5-HT, DA (in some insects), OA that have been associated with broad physiological states like waking state, aversion, attention 
and learning/memory (McLean and Shipley, 1987; Mandairon et al., 2006; Matsutani and Yamamoto, 2008; Fletcher and Chen, 2010; Wasserman et al., 2013; but see Linster and Cleland, 2016). Here, I focus on the known mechanisms and effects of 5-HT and DA on olfactory coding as the AL receives 5-HT innervation from extrinsic sources and DA alters olfactory processing via both intrinsic (in some insect ALs) and extrinsic modulation.

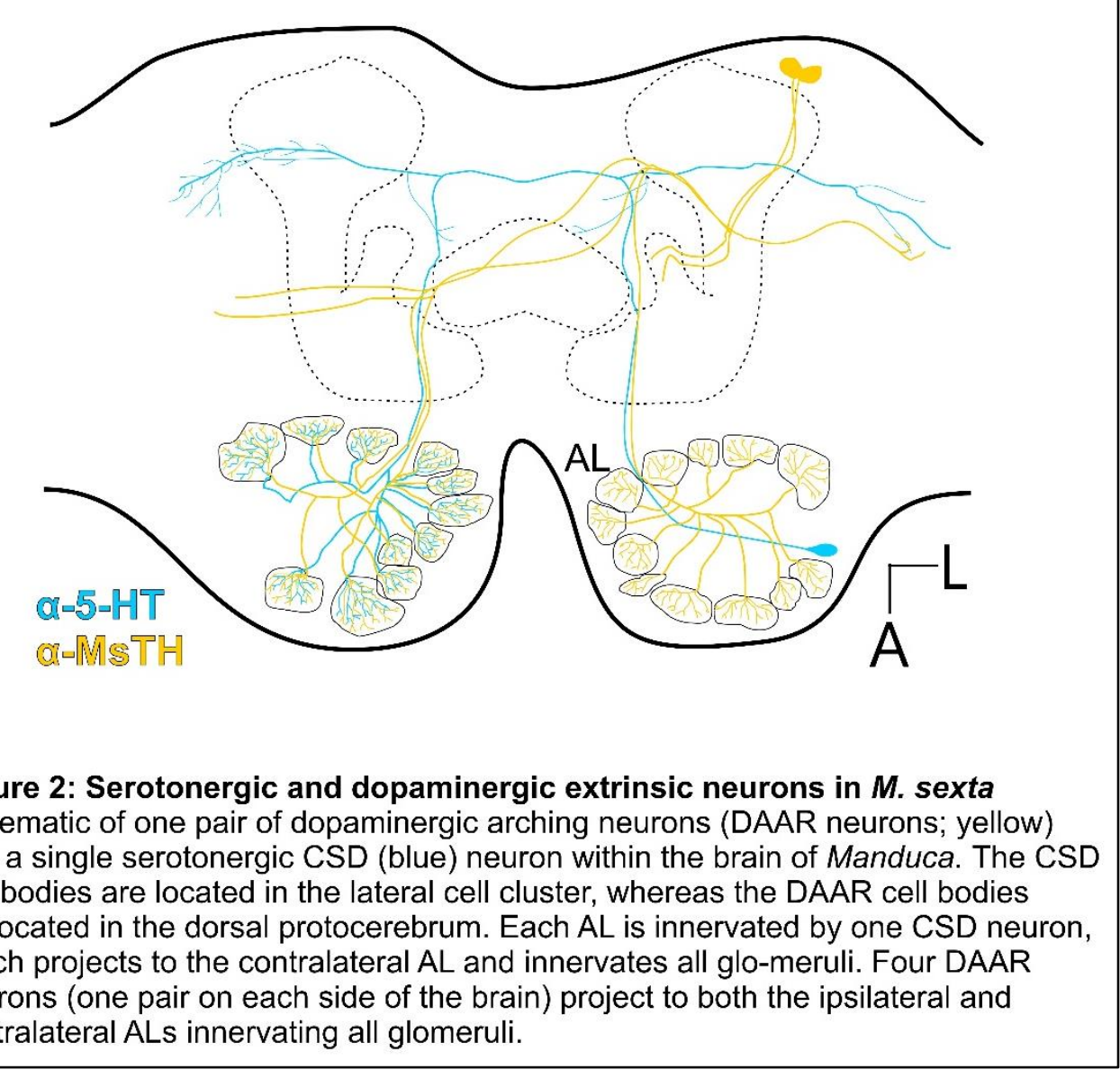

Cell class specific effects of serotonergic modulation

In the $\mathrm{AL}$ and $\mathrm{OB}$, neuronal class specific 5-HT receptor expression results in relatively heterogeneous effects of 5-HT, even within the same neuronal class. Each AL across a wide range of insects typically receives input from 1-2 serotonergic neurons (Kent et al., 1987; Salecker and Distler, 1990; Wegerhoff, 1999; Ignell, 2001; Dacks et al., 2006; Roy et al., 2007) that widely innervate all glomeruli. However, despite the ubiquity of 5-HT in the olfactory systems across insects, the consequences of serotonergic modulation of olfaction have been remarkably uneven 
across model systems and behavioral tasks. In moths, 5-HT enhances behavioral attraction to sex pheromone (Linn and Roelofs, 1986; Gatellier et al., 2004; Kloppenburg and Mercer, 2008), suggesting that 5-HT upregulates olfactory sensitivity. However, studies directly manipulating serotonergic neurons or serotonergic signaling in the olfactory system indicate that the role of 5HT is more complex. For instance, conditionally eliminating tryptophan hydroxylase 2 expression in the raphe of mice, and therefore 5-HT synthesis after olfactory development, had no effect on performance in several general olfactory behavioral assays (Carlson et al., 2016). In Drosophila, suppressing the activity of the serotonergic neurons in the AL (the "CSDns") increases $\mathrm{CO}_{2}$ avoidance, while blocking synaptic transmission decreases sensitivity to the pheromone cVA (Singh et al., 2013), suggesting that the effects of 5-HT can be odor dependent. Furthermore, the CSDns modulate ethanol attraction in concert with other serotonergic neurons that do not innervate the AL (Xu et al., 2016).

Similar to behavioral studies, the physiological effects of 5-HT within the olfactory system are also heterogeneous. In moths, bath applied 5-HT reduces two $\mathrm{K}^{+}$conductances (Mercer et al., 1995, 1996; Kloppenburg et al., 1999), enhancing PN and LN excitability resulting in increased odor evoked activity (Kloppenburg et al., 1999; Dacks et al., 2008). However, this only occurs for roughly half of the neurons recorded in these studies, and in some instances 5-HT decreases odor evoked responses in an odor-dependent manner (Kloppenburg et al., 1999; Dacks et al., 2008). In Drosophila, bath application of 5-HT enhances PN odor-evoked responses and sensitivity (Dacks et al., 2009; Zhang and Gaudry, 2016). However, endogenous release of 5-HT from the CSDns reduces PN odor-evoked responses in the AL (Zhang and Gaudry, 2016). Surprisingly, the sole source of serotonergic innervation to the AL (the CSDns) do not affect PN responses to cVA, yet other serotonergic neurons outside the AL do affect cVA responses (Zhang and Gaudry, 2016). These results suggest that the AL can be modulated by both synaptic and non-synaptic sources of 5-HT, perhaps via the hemolymph, in an odor-dependent manner.

The heterogeneous effects of 5-HT in the olfactory system likely arise due to cell-type specific 5-HT receptor expression (Tierney 2018) and the heterogeneity of serotonergic neurons innervating the AL and olfactory bulb (Ren et al., 2018). All five insect 5-HT receptors are expressed in the ALs of Drosophila (Sizemore and Dacks, 2016) and Manduca (Dacks et al., 2013). In Drosophila, each neuronal class expresses a different combination of 5-HT receptors. 
However, any given receptor is only expressed by a subset of neurons within that class (Sizemore and Dacks, 2016) which likely contributes to the non-uniform effects of 5-HT. Consequently, complex receptor expression patterns likely play a major role in the observed heterogeneity in the effects of 5-HT. Heterogeneity of serotonergic neurons also likely contribute to the non-uniform effects of 5-HT in the olfactory system. Serotonergic neurons innervate different functional zones within the olfactory system of vertebrates (Won et al., 1998; Gómez et al., 2005; Steinfeld et al., 2015) and even different functional zones within glomeruli in Manduca (Sun et al., 1993; Lizbinski et al., 2016). Glomerular specific differences in serotonergic innervation have also been observed in the OB (Gómez et al., 2005), and the processes of the CSDns in Drosophila (Singh et al., 2013). Furthermore, the distribution of CSDn active zones vary widely across glomeruli, yet are highly stereotyped across individual animals (Coates et al., 2017). Thus, even within a single identified modulatory neuron, specific traits can be heterogeneous across compartments. In addition, the CSDns receive network wide inhibition from LNs and glomerulus-specific excitation from OSNs and PNs, indicating that 5-HT modulation cannot be considered purely "Top-Down" (Coates et al., 2017). Since the CSDns receive input based on AL network dynamics as well as from other networks, they can be considered partially intrinsic to the AL. Finally, the circumstances in which 5-HT is released are surprisingly varied (Andrade and Haj-Dahmane, 2013; Dayan and Huys, 2015). The levels of 5-HT in the AL of moths fluctuate throughout the day, peaking when moths are most active (Kloppenburg et al., 1999) reminiscent of daily fluctuations of Raphe neuron activity and 5-HT production (Trulson and Jacobs, 1979; Jacobs and Fornal, 1991; Park et al., 1999; Corthell et al., 2013). The heterogeneous nature of serotonergic neurons and the complicated context in which 5-HT is released likely contribute to the non-uniform effects that have been observed for the physiological and behavioral consequences of 5-HT.

\section{Dopaminergic modulation in a broader context}

While less is known about the consequences of extrinsic dopaminergic modulation in comparison to 5-HT, some mechanisms have been identified in olfactory neurons of a few insect species. Similar to serotonergic modulation, dopaminergic modulation is heterogeneous across multiple parameters within both the olfactory system and across organisms (Vinauger et al., 2018, Dacks et al., 2012). While some insect LNs also release DA (Kirchof et al., 1999; Hamanaka et al., 2016; Tedjakumala et al., 2017), the sole source of DA in the AL of M. sexta comes from 
centrifugal neurons called the dopaminergic arching neurons (DAARs, as seen in Figure 2) which act as extrinsic modulators in the context of aversion (Dacks et al., 2012). In M. sexta, the DAARs innervate all glomeruli, however in other insects like the mosquito, the degree to which each glomerulus was innervated by DA varied (Vinauger et al., 2018). This suggests that DA may selectively modulate certain odors more (or less) than others.

Within the AL of M. sexta, DA is associated with the context of aversive stimuli, as DA receptor antagonists injected into the AL block aversive (Dacks et al., 2012), but not appetitive conditioning (Riffell et al., 2013). In mosquitos, blocking excitatory D1 receptors prevents olfactory learning (Vinauger et al., 2018). This effect is likely mediated by both the AL and the mushroom bodies, the center for learning and memory in insects, as dopamine plays a central role in olfactory learning (Unoki et al., 2005; Schwaerzel et al 2003; Kim et al, 2007; Schroll et al., 2006). In vivo studies of cultured honeybee AL neurons demonstrate that DA modulates $\mathrm{Ca}^{2+}$ dependent $\mathrm{K}^{+}$currents, thereby increasing the excitability of these neurons (Perk et al., 2006). The effects of DA may be cell-type specific however, as Perk et.al. only recorded from one type of AL neuron. In M. sexta DA increased the maximal odor-evoked firing rate of many extracellularly recorded AL neurons, but did not affect response gain (Dacks et al., 2012) as with 5-HT (Dacks et al., 2008). DA and 5-HT also differ in their effects on the temporal dynamics of odor-evoked responses. While 5-HT generally increases odor evoked response duration (Kloppenburg et al., 1999; Dacks et al., 2008), DA reduced the post-excitation period of spike suppression in PNs called the I2 phase (Dacks et al., 2012) that typically lasts between 10ms and 1.5 seconds (Christensen et al., 1998). The I2 phase affects the fidelity with which PNs can encode the temporal dynamics of odor stimuli and blocking the I 2 phase altogether negatively impacts the ability of moths to track odor plumes (Lei et al., 2009; Tripathy et al., 2010), suggesting that DA may modulate temporal pattern of odor-evoked responses of AL neurons. Dopamine also selectively modulates mosquito AL neurons, as bath application of DA both increased and decreased odor-evoked responses (Vinauger et al., 2018). Furthermore, the degree to which each AL neuron was modulated by DA differed across odors, suggesting that dopaminergic extrinsic modulation may be odor-specific (Vinauger et al., 2018).

This heterogeneity in DA responses is likely due to cell-class specific receptor expression within populations of AL neurons. The mRNAs for three of the four known DA receptors the 
MsDop1, MsDop2 and MsINDR receptors are expressed in the AL (Dacks et al., 2012). There is a fourth insect DA receptor, the DA ecdysteroid receptor (Abrieux et al., 2014), however the expression patterns in M. sexta are unknown. Altogether, receptor expression by specific neuronal types of the AL (i.e. LN vs. PN) of $M$. sexta is still relatively unexplored. DA receptor activation has the capacity to converge upon the same biochemical cascades as all DA receptors couple to adenylyl cyclase to affect cAMP levels (Saudou et al., 1992; Colas et al., 1995; Mustard et al., 2005), however the D1 and D2 receptors have opposing effects. Overall, prescribing a unified role for dopamine is impossible because the context in which DA is released is unknown.

\section{Intrinsic Modulation via Olfactory Local Interneurons:}

The olfactory system must efficiently encode odorant information over a wide concentration range to produce reliable representations of odor identity. Heterogeneous populations of LNs alter the input/output relationship of principal cell types to accomplish much of this computation. LNs are the main source of intrinsic modulation in the antennal lobe and perform many tasks including presynaptic gain control (Olsen and Wilson, 2008; Root et al., 2008; Wang, 2012), divisive normalization (Olsen et al., 2010), lateral excitation (Olsen et al., 2007) and inhibition (Olsen and Wilson, 2008), regulating spike timing by promoting synchrony (Christensen et al., 2000; Lei et al., 2002; Schoppa, 2006), and modulating dynamic control of response range (reviewed in Martin et al., 2011 and Wilson et al, 2013). LNs are particularly heterogeneous and those found in insect olfactory systems are no exception (Nässel and Homberg, 2006). LNs can release a variety of transmitters including many neuromodulators that act over a range of timescales and in Drosophila, lateral input from inhibitory LNs scales with overall network activity (Olsen and Wilson, 2008). Thus, LNs intrinsically modulate odor coding within the context of current and previous network activation. Intrinsic modulation of olfactory processing via local interneurons can 1) alter network output based on the strength of odor input 2) mediate long-lasting temporal effects via activation of metabotropic receptors 3) regulate the dynamic range of output neurons, allowing for reliable coding of odor identity across a range of stimulus intensities. 4) regulate spike timing, promoting synchrony between AL neurons and 4) mediate contrast enhancement via lateral excitation and inhibition. 

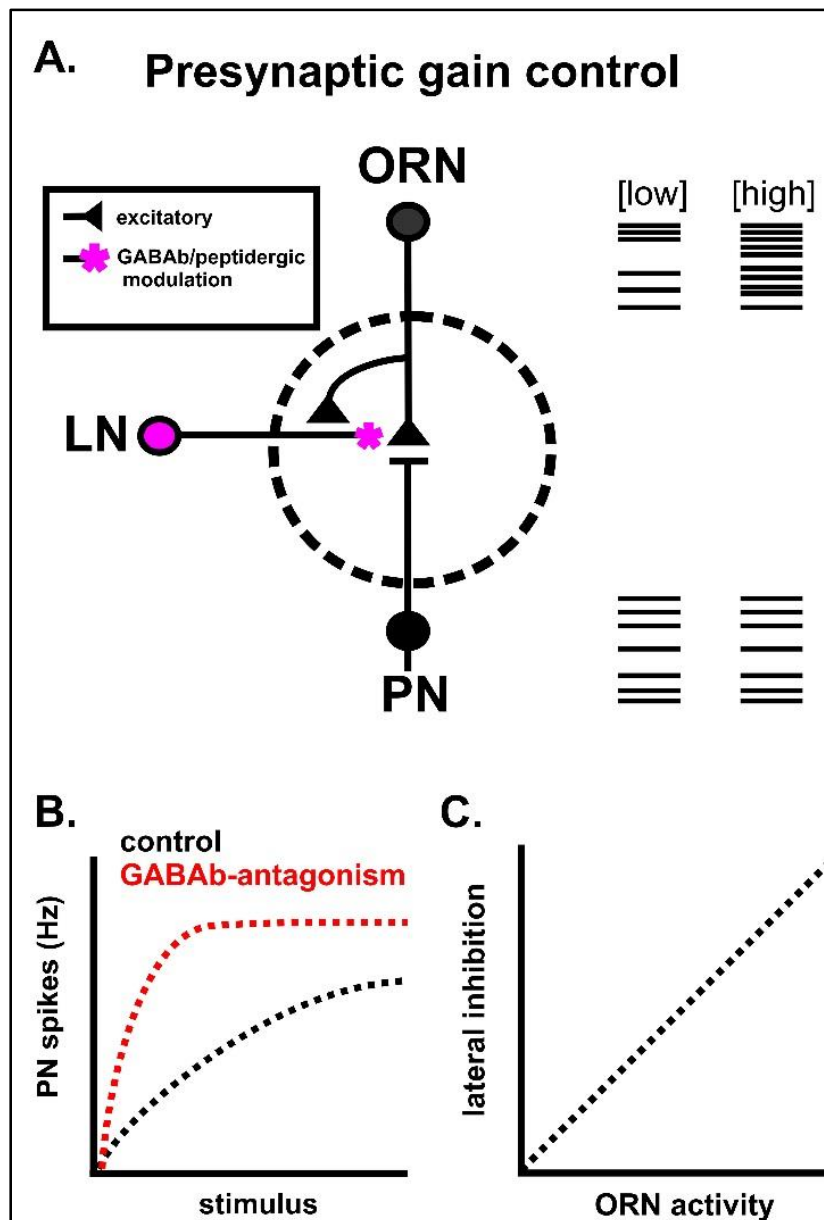

C.

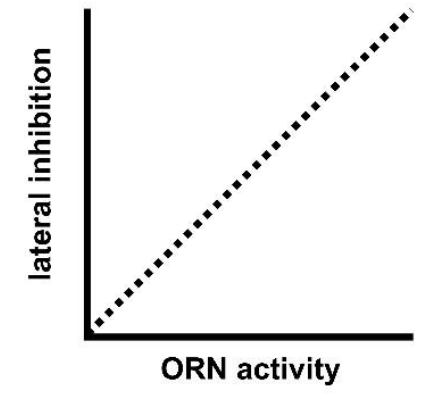

Figure 3: Intrinsic modulation as a means of presynaptic gain control. (A) Presynaptic gain control alters the signal strength between ORNs and PNs to filter out noise from spontaneous firing of ORNs, avoid PN saturation and expand the dynamic range of PNs. It does this by reducing presynaptic calcium levels in ORNs via GABAb and neuropeptide receptors, and reducing the likelihood of acetycholine release onto PNs. (B) GABAb blockade (red) increases presynaptic calcium influx and decreases the range of ORN input over which PN firing rate can change, ultimately resulting in $\mathrm{PN}$ saturation (based on results from Root et al., 2008; Olsen et al., 2010). (C) GABAergic lateral inhibition scales with network activation (as measured by ORN activity; Olsen and Wilson, 2008).
The information transferred from individual OSNs to PNs is highly reliable (Murphy et al., 2004) yet non-linear (Wilson et al., 2004; Bhandawat et al., 2007; Olsen and Wilson, 2008). At high stimulus intensities, output neuron activity can saturate such that further increases in OSN output do not result in a concomitant increase in output neuron activity. To avoid saturation, the olfactory system relies on presynaptic inhibition as a means of gain control. Gain control adjusts the signal strength between input and output neurons to filter out noise from spontaneous firing of ORNs (Wilson, 2013), avoid saturation (Olsen and Wilson, 2008; Root et al., 2008; Martin et al., 2011) and adjust the dynamic range of output neurons (Olsen and Wilson, 2008; Root et al., 2008). It does this, broadly, by activating $\mathrm{GABAb}$ receptors on presynaptic terminals to reduce presynaptic $\mathrm{Ca}^{2+}$ levels, resulting in reduced transmitter release from the presynaptic neuron (Figure 3) (Wang, 2012). While early studies on GABAergic inhibition in the insect AL focused on the role of ionotropic GABAa mediated lateral inhibition (Waldrop et al., 1987; Christensen et al., 1993, 1998a,b; Lei et al., 2002), GABAa blockade in insects does not fully block a slower form of inhibition (MacLeod and Laurent, 1996; Christensen et al., 1998b; Bazhenov et al., 2001; Wilson 
et al., 2004; Wilson and Laurent, 2005). Together this suggested that 1) GABA may also act presynaptically and 2) a slower, metabotropic mechanism is at play.

In Drosophila melanogaster, GABAb blockade on OSN terminals increases presynaptic $\mathrm{Ca}^{2+}$ influx, broadens odor tuning of PNs and decreases the range of ORN input over which PN firing rate can change, ultimately resulting in PN saturation (Figure 3B) (Olsen and Wilson, 2008; Root et al., 2008). In normal conditions, PN responses are normalized via increased lateral inhibition which scales with ORN activity (Figure 3C) (Olsen et al., 2010). Overall, this suggests that interglomerular presynaptic inhibition adjusts the dynamic range of PNs to avoid saturation and refines the breadth of odor tuning across a wide range of stimulus intensities (Wang, 2012). It is important to note that GABA is not the sole modulator of gain control in insects, as the neuropeptides tachykinin, (Ignell et al., 2009) and short neuropeptide F (Root et al., 2011; Ko et al., 2015) also mediate presynaptic inhibition.

While presynaptic inhibition is ubiquitous, it is not exerted evenly across the olfactory system. In Drosophila, some glomeruli are more subject to inhibition than others simply due to differences in glomerulus-specific LN innervation (Wilson and Laurent, 2005; Chou et al., 2010) and OSN GABAb receptor expression (Root et al., 2008). This suggests that specific odors differ in the amount of "shelter" they need from ongoing activity in the olfactory system, and are therefore insulated from presynaptic gain control. Furthermore, LNs are heterogeneous in their morphology, physiology and transmitter content (Seki and Kanzaki, 2008; Carlsson et al., 2010; Chou et al., 2010; Seki et al., 2010; Reisenman et al., 2011; Nagayama et al., 2014) making it challenging to determine the sub-populations of neurons involved and the mechanisms by which they perform these tasks.

\section{Local interneuron heterogeneity and peptidergic modulation}

In Manduca, as well as other insects, AL LNs express a wide array of neurotransmitters including a combination of GABA and multiple neuropeptides (Homberg et al., 1990; Schachtner et al., 2004; Utz et al., 2007; Utz et al., 2008; Reisenmann et al., 2011; Fusca et al., 2015). Consequently, understanding the functional roles of individual LNs is complex as we lack a systematic analysis of transmitter co-expression (Nässel, 2018). For example, LNs may not consistently express the same combination of transmitters, resulting a heterogeneous cocktail of neuromodulators from even single LNs. Furthermore, the presence of specific sets of 
neuropeptides or combinations of transmitter types allows LNs to have a combinatorial impact on their targets by affecting either ionotropic and/or metabotropic G-protein coupled receptors (Nusbaum et al., 2001; Tritsch et al., 2016; Nusbaum et al., 2017). Additionally, the effects $\mathrm{GABA}_{\mathrm{B}}$ receptor activation are far shorter-lasting relative to neuropeptide receptors (Salio et al., 2006). A neuron that releases both GABA and a neuropeptide can mediate fast inhibition (via GABAa receptors), slow neuromodulation (via GABAb receptors) and even longer lasting peptidergic modulation, resulting in layered effects over different timescales on its downstream targets. For example, a single modulatory neuron in the stomatogastric ganglion, MCN1, releases GABA and two neuropeptides which mediate divergent effects with varying time scales, on different neurons in the network (Stein et al 2007). Furthermore, neuropeptide receptors can be excitatory (Horodyski et al., 2011) or inhibitory (Yapici et al., 2008; Ignell et al., 2009; Asahina et al., 2014; Ko et al., 2015), suggesting that peptide co-release by a single LN could mediate a complex mix of excitation and inhibition.

As a population, LNs are tonically, spontaneously active (Lei et al 2011) even without odor-evoked network activity. However, the network may need to be more strongly activated (i.e. by higher concentrations of odors or increased length of odor-stimuli) for LNs to release neuropeptides due to the different calcium binding affinities of distinct synaptotagmins associated with small clear vesicles and dense-core vesicles (Saraswati et al., 2007; Bacaj et al., 2013; Fukuda et al., 2004). Thus, the consequences of LN activation and peptidergic modulation may depend more upon the degree of network activity rather than the identity of any singular LN that is activated. Overall, this heterogeneous cocktail of peptides likely provides the AL a flexible range of states to up or down regulate olfactory processing over a variety of time frames and spatial scales within the context of ongoing network activity. Thus, while LNs function as intrinsic modulators of olfactory coding, a variety of mechanisms make their influence non-uniform. Ultimately, heterogeneous transmitter co-release in LNs provides a powerful means for circuit flexibility, allowing them to modulate other neurons within the context of previous and ongoing network activity.

\section{The Integration of Multiple Modulators:}

The potential for integrated modulatory influence is present in all neural networks. For example, the stomatogastric ganglion, a central pattern generator in the crab, Cancer borealis, is 
bathed in a dynamic combination of over 25 modulators (Marder, 2012). The neurons in the circuit must therefore integrate the influence of multiple transmitters on a variety of a spatial, temporal and molecular scales, resulting in exponential possibilities for a circuit to alter its functional connectivity resulting in unique changes to network output. Multiple modulators could 1) converge onto the same neurons (reviewed in Marder, 2012; Nadim and Bucher, 2014), 2) target common channels intrinsic to neurons, thereby altering excitability in the same manner, 3) converge (or diverge) onto the same (or opposing) second messenger cascades (Brezina et al., 1996; Mesce et al., 2001), and finally 5) modulate surrounding neurons in the network resulting in complex polysynaptic effects (Katz and Frost, 1995). While the mechanisms by which multiple modulators are integrated have been explored in motor systems (Blitz et al., 1999; Brezina et al., 1996; Mesce et al., 2001; Thirumalai and Marder, 2002; Beliez et al., 2014), this phenomenon has been virtually unexplored in sensory systems.

In the insect AL, most, if not all glomeruli receive input from 5-HT (Kent et al., 1987; Salecker and Distler, 1990; Sun et al., 1993; Wegerhoff, 1999; Hill et al., 2002; Dacks et al., 2006a; Roy et al., 2007), DA (Dacks et al 2013; Vinauger et al 2018), and octopamine (Dacks et al., 2005; Sinakevitch et al., 2005; Sinakevitch and Strausfeld, 2006), and local interneurons across many species release GABA and a wide-array of neuropeptides (Carlsson et al., 2010; Kromann et al., 2013; Siju et al., 2013; Fusca at el., 2015 Lizbinski et al., 2018). In the AL of M. sexta, the CSD and DAAR neurons both ramify in all glomeruli (Kent et al., 1987; Dacks et al., 2006a; Dacks et al., 2012) however, their relative projection patterns reveal the potential for integrated modulation on LNs and PNs and differences in their ability to presynaptically modulate ORNs. Furthermore, the wide-array of transmitters released from LNs (Lizbinski et al., 2018) provides multiple mechanisms by which intrinsic modulators can alter every stage of olfactory processing within the AL. Therefore, AL neurons must integrate the influence of both extrinsic and intrinsic sources of modulation in order to produce flexible output based on the current physiological state of the animal.

While neuromodulation is ubiquitous, the mechanisms by which single neurons integrate the influence of multiple modulators is completely underexplored. In order to understand realistic network function, network processing must be considered within the context of the dynamic cocktail of modulators released by both intrinsic and extrinsic modulatory neurons. In this 
dissertation, I address three main questions regarding the integration of intrinsic and extrinsic modulators in the olfactory system. I begin by determining whether two extrinsic modulators, serotonin and dopamine, affect the same principal olfactory neurons using anatomy (Chapter 2) and physiology (Chapter 3). I then determine the integrated effects of both modulators on population of AL neurons and ask whether they modulate olfactory processing via the same mechanisms (Chapter 3). Finally, due to the heterogeneity of LNs and the variety of transmitters they co-release it is difficult to determine the mechanisms by which local interneurons modulate olfactory processing. To this end, I systemically analyze transmitter co-expression in LNs (the source of intrinsic modulation in the AL) to determine the organizing principles of local interneuron heterogeneity (Chapter 4). Altogether, my work details the organizing principles that govern the integration of multiple neuromodulators on both a single neuron and network widebasis in the olfactory system.

\section{Works Cited:}

Abrieux, A., Duportets, L., Debernard, S., Gadenne, C., \& Anton, S. (2014). The GPCR membrane receptor, DopEcR, mediates the actions of both dopamine and ecdysone to control sex pheromone perception in an insect. Frontiers in behavioral neuroscience, 8, 312.

Andrade, R., and Haj-Dahmane, S. (2013). Serotonin neuron diversity in the dorsal raphe. ACS Chem. Neurosci. 4, 22-25. doi: 10.1021/cn300224n

Asahina, K., Watanabe, K., Duistermars, B. J., Hoopfer, E., González, C. R., Eyjólfsdóttir, E. A., ... \& Anderson, D. J. (2014). Tachykinin-expressing neurons control male-specific aggressive arousal

in Drosophila. Cell, 156(1), 221-235.

Bacaj, T., Wu, D., Yang, X., Morishita, W., Zhou, P., Xu, W., ... \& Südhof, T. C. (2013). Synaptotagmin-

1 and synaptotagmin-7 trigger synchronous and asynchronous phases of neurotransmitter release. Neuron, 80(4), 947-959.

Bazhenov, M., Stopfer, M., Rabinovich, M., Abarbanel, H. D., Sejnowski, T. J., and Laurent, G. (2001). Model of cellular and network mechanisms for odor-evoked temporal patterning in the locust antennal lobe. Neuron 30, 569-581. doi: 10.1016/s0896-6273(01)00286-0

Beliez, L., Barrière, G., Bertrand, S. S., \& Cazalets, J. R. (2014). Multiple monoaminergic modulation of posturo-locomotor network activity in the newborn rat spinal cord. Frontiers in neural circuits, 8 , 99.

Berg, B. G., Schachtner, J., Utz, S., and Homberg, U. (2007). Distribution of neuropeptides in the primary olfactory center of the heliothine moth Heliothis virescens. Cell Tissue Res. 327, 385-398. doi: 1 0.1007/s00441-006-0318-X

Bhandawat, V., Olsen, S. R., Gouwens, N. W., Schlief, M. L., and Wilson, R. I. (2007). Sensory processing in the Drosophila antennal lobe increases reliability and separability of ensemble odor representations. Nat. Neurosci. 10, 1474-1482. doi: 10.1038/nn1976 
Blitz, D. M., \& Nusbaum, M. P. (1999). Distinct functions for cotransmitters mediating motor pattern selection. Journal of Neuroscience, 19(16), 6774-6783.

Brezina, V., Orekhova, I. V., \& Weiss, K. R. (1996). Functional uncoupling of linked neurotransmitter effects by combinatorial convergence. Science, 273(5276), 806-810.

Carlson, K. S., Whitney, M. S., Gadziola, M. A., Deneris, E. S., and Wesson, D. W. (2016). Preservation of essential odor-guided behaviors and odor-based reversal learning after targeting adult brain serotonin synthesis. eNeuro 3:ENEURO.0257-16.2016. doi: 10.1523/ENEURO.0257-16.2016

Carlsson, M. A., Diesner, M., Schachtner, J., and Nässel, D. R. (2010). Multiple neuropeptides in the Drosophila antennal lobe suggest complex modulatory circuits. J. Comp. Neurol. 518, 33593380. doi: 10.1002/cne.22405

Chou, Y. H., Spletter, M. L., Yaksi, E., Leong, J. C., Wilson, R. I., and Luo, L. (2010). Diversity and wiring variability of olfactory local interneurons in the Drosophila antennal lobe. Nat. Neurosci. 13, 439-449. doi: 10.1038/nn.2489

Christensen, T. A., Pawlowski, V. M., Lei, H., \& Hildebrand, J. G. (2000). Multi-unit recordings reveal context-dependent modulation of synchrony in odor-specific neural ensembles. Nature neuroscience, 3(9), 927.

Christensen, T. A., Waldrop, B. R., \& Hildebrand, J. G. (1998). Multitasking in the olfactory system: context-dependent responses to odors reveal dual GABA-regulated coding mechanisms in single olfactory projection neurons. Journal of Neuroscience, 18(15), 5999-6008.

Christensen, T. A., Waldrop, B. R., Harrow, I. D., and Hildebrand, J. G. (1993). Local interneurons and information processing in the olfactory glomeruli of the moth Manduca sexta. J. Comp. Physiol. A 173, 385-399. doi: 10.1007/bf00193512

Christensen, T. A., Waldrop, B. R., and Hildebrand, J. G. (1998a). GABAergic mechanisms that shape the temporal response to odors in moth olfactory projection neurons. Ann. N Y Acad. Sci. 855, 475481. doi: 10.1111/j.1749-6632.1998.tb10608.x

Colas, J. F., Launay, J. M., Kellermann, O., Rosay, P., \& Maroteaux, L. (1995). Drosophila 5-HT2 serotonin receptor: coexpression with fushi-tarazu during segmentation. Proceedings of the National Academy of Sciences, 92(12), 5441-5445.

Coates, K. E., Majot, A. T., Zhang, X., Michael, C. T., Spitzer, S. L., Gaudry, Q., et al. (2017). Identified serotonergic modulatory neurons have heterogeneous synaptic connectivity within the olfactory system of Drosophila. J. Neurosci. 37, 7318-7331. doi: 10.1523/JNEUROSCI.0192-17.2017

Corthell, J. T., Stathopoulos, A. M., Watson, C. C., Bertram, R., and Trombley, P. Q. (2013). Olfactory

bulb monoamine concentrations vary with time of day. Neuroscience 247, 234-241. doi: 10.1016/j.neuroscience.2013.05.040

Dacks, A. M., Christensen, T. A., Agricola, H. J., Wollweber, L., and Hildebrand, J. G. (2005).

Octopamine- immunoreactive neurons in the brain and subesophageal ganglion of the hawkmoth Manduca $\quad$ sexta. J. Comp. Neurol. 488, 255-268. doi: 10.1002/cne.20556 
Dacks, A. M., Christensen, T. A., and Hildebrand, J. G. (2006). Phylogeny of a serotonin-immunoreactive neuron in the primary olfactory center of the insect brain. J. Comp. Neurol. 498, 727-746. doi: 10.1002/cne.21076

Dacks, A. M., Christensen, T. A., and Hildebrand, J. G. (2008). Modulation of olfactory information processing in the antennal lobe of Manduca sexta by serotonin. J. Neurophysiol. 99, 2077-2085. doi: 10.1152/jn.01372.2007

Dacks, A. M., Riffell, J. A., Martin, J. P., Gage, S. L., \& Nighorn, A. J. (2012). Olfactory modulation by dopamine in the context of aversive learning. Journal of neurophysiology, 108(2), 539-550.

Dacks, A. M., Siniscalchi, M. J., and Weiss, K. R. (2012b). Removal of default state-associated inhibition during repetition priming improves response articulation. J. Neurosci. 32, 17740-17752. doi: 10.1523/JNEUROSCI.4137-12.2012

Dacks, A. M., Reale, V., Pi, Y., Zhang, W., Dacks, J. B., Nighorn, A. J., et al. (2013). A characterization of the manduca sexta serotonin receptors in the context of olfactory neuromodulation. PLoS One 8:e69422. doi: 10.1371/journal.pone.0069422

Dayan, P., and Huys, Q. (2015). Serotonin's many meanings elude simple theories. Elife 4:e07390. doi: 10.7554/eLife.07390

Fletcher, M. L., and Chen, W. R. (2010). Neural correlates of olfactory learning: critical role of centrifugal neuromodulation. Learn. Mem.17, 561-570. doi: 10.1101/1m.941510

Friedman, A. K., and Weiss, K. R. (2010). Repetition priming of motoneuronal activity in a small motor network: intercellular and intracellular signaling. J. Neurosci. 30, 8906-8919. doi: 10.1523/JNEUROSCI.1287-10.2010

Fukuda, M., Kanno, E., Satoh, M., Saegusa, C., \& Yamamoto, A. (2004). Synaptotagmin VII is targeted to dense-core vesicles and regulates their Ca2+-dependent exocytosis in PC12 cells. Journal of Biological Chemistry, 279(50), 52677-52684.

Fusca, D., Schachtner, J., and Kloppenburg, P. (2015). Colocalization of allatotropin and tachykininrelated peptides with classical transmitters in physiologically distinct subtypes of olfactory local interneurons in the cockroach (Periplaneta americana). J. Comp. Neurol. 523, 1569-1586. doi: $10.1002 /$ cne. 23757

Gatellier, L., Nagao, T., and Kanzaki, R. (2004). Serotonin modifies the sensitivity of the male silkmoth to pheromone. J. Exp. Biol. 207, 2487-2496. doi: 10.1242/jeb.01035

Goldman, A. L., Van der Goes van Naters, W., Lessing, D., Warr, C. G., and Carlson, J. R. (2005). Coexpression of two functional odor receptors in one neuron. Neuron 45, 661-666. doi: 10.1016/j.neuron.2005.01.025

Gómez, C., Briñón, J. G., Barbado, M. V., Weruaga, E., Valero, J., and Alonso, J. R. (2005). Heterogeneous targeting of centrifugal inputs to the glomerular layer of the main olfactory bulb. $J$. Chem. Neuroanat. 29, 238-254. doi: 10.1016/j.jchemneu.2005.01.005

Gupta, N., and Stopfer, M. (2011). Insect olfactory coding and memory at multiple timescales. Curr. Opin. Neurobiol. 21, 768-773. doi: 10.1016/j.conb.2011.05.005 
Hamanaka, Y., Minoura, R., Nishino, H., Miura, T., and Mizunami, M. (2016). Dopamine- and tyrosine hydroxylase-immunoreactive neurons in the brain of the american cockroach, Periplaneta americana. PLoS One 11:e0160531. doi: 10.1371/journal.pone.0160531

Harris-Warrick, R. M., and Marder, E. (1991). Modulation of neural networks for behavior. Annu. Rev. Neurosci. 14, 39-57. doi: 10.1146/annurev.neuro.14.1.39

Homberg, U., Kingan, T. G., and Hildebrand, J. G. (1990). Distribution of FMRFamide-like immunoreactivity in the brain and suboesophageal ganglion of the sphinx moth Manduca sexta and colocalization with SCPB-, BPP-, and GABA-like immunoreactivity. Cell Tissue Res. 259,

401- 419. doi: 10.1007/bf01740767

Horodyski, F. M., Verlinden, H., Filkin, N., Vandersmissen, H. P., Fleury, C., Reynolds, S. E., ... \& Broeck, J. V. (2011). Isolation and functional characterization of an allatotropin receptor from Manduca $\quad$ sexta. Insect biochemistry and molecular biology, 41(10), 804-814.

Ignell, R. (2001). Monoamines and neuropeptides in antennal lobe interneurons of the desert locust, Schistocerca gregana: an immunocytochemical study. Cell Tissue Res. 306, 143-156. doi: $10.1007 / \mathrm{s} 004410100434$

Ignell, R., Root, C. M., Birse, R. T., Wang, J. W., Nässel, D. R., and Winther, A. M. (2009). Presynaptic peptidergic modulation of olfactory receptor neurons in Drosophila. Proc. Natl. Acad. Sci. U S A 106, 13070-13075. doi: 10.1073/pnas.0813004106

Jacobs, B. L., and Fornal, C. A. (1991). Activity of brain serotonergic neurons in the behaving animal. Pharmacol. Rev. 43, 563-578.

Joseph, R. M., and Carlson, J. R. (2015). Drosophila chemoreceptors: a molecular interface between the chemical world and the brain. Trends Genet. 31, 683-695. doi: 10.1016/j.tig.2015.09.005

Kaczmarek, L. K., and Levitan, I. B. (1987). Neuromodulation: The Biochemical Control of Neuronal Excitability. New York, NY: Oxford University Press.

Katz, P. S. (1995). Intrinsic and extrinsic neuromodulation of motor circuits. Curr. Opin. Neurobiol. 5, 799-808. doi: 10.1016/0959-4388(95)80109-х

Katz, P. S., and Frost, W. N. (1995). Intrinsic neuromodulation in the Tritonia swim CPG: serotonin mediates both neuromodulation and neurotransmission by the dorsal swim interneurons. $J$. Neurophysiol. 74, 2281-2294.

Katz, P. S., and Frost, W. N. (1996). Intrinsic neuromodulation: altering neuronal circuits from within. Trends Neurosci. 19, 54-61. doi: 10.1016/0166-2236(97)90029-1

Katz, P. S. (1999). Beyond Neurotransmission: Neuromodulation and its Importance for Information Processing. New York, NY: Oxford University Press.

Kent, K. S., Hoskins, S. G., and Hildebrand, J. G. (1987). A novel serotonin-immunoreactive neuron in the antennal lobe of the sphinx moth Manduca sexta persists throughout postembryonic life. $J$. Neurobiol. 18, 451-465. doi: 10.1002/neu.480180506

Kim, Y. C., Lee, H. G., \& Han, K. A. (2007). D1 dopamine receptor dDA1 is required in the mushroom body neurons for aversive and appetitive learning in Drosophila. Journal of Neuroscience, 27(29), 7640-7647. 
Kirchhof, B. S., Homberg, U., and Mercer, A. R. (1999). Development of dopamine-immunoreactive neurons associated with the antennal lobes of the honey bee, Apis mellifera. J. Comp.

Neurol. 411, 643-653. doi: 10.1002/(sici)1096-9861(19990906)411:4<643::aid-cne8>3.0.co;2-o

Kloppenburg, P., Ferns, D., and Mercer, A. R. (1999). Serotonin enhances central olfactory neuron responses to female sex pheromone in the male sphinx moth manduca sexta. J. Neurosci. 19, 8172-8181.

Kloppenburg, P., and Mercer, A. R. (2008). Serotonin modulation of moth central olfactory neurons. Annu. Rev. Entomol. 53, 179-190. doi: 10.1146/annurev.ento.53.103106.093408

Ko, K. I., Root, C. M., Lindsay, S. A., Zaninovich, O. A., Shepherd, A. K., Wasserman, S. A., ... \& Wang, J. W. (2015). Starvation promotes concerted modulation of appetitive olfactory behavior via parallel neuromodulatory circuits. Elife, 4, e08298.

Koh, H. Y., and Weiss, K. R. (2005). Peptidergic contribution to posttetanic potentiation at a central synapse of aplysia. J. Neurophysiol.94, 1281-1286. doi: 10.1152/jn.00073.2005

Koh, H. Y., and Weiss, K. R. (2007). Activity-dependent peptidergic modulation of the plateaugenerating neuron B64 in the feeding network of Aplysia. J. Neurophysiol. 97, 1862-1867. doi: $10.1152 /$ jn.01230.2006

Kupfermann, I. (1979). Modulatory actions of neurotransmitters. Annu. Rev. Neurosci. 2, 447-465. doi: 10.1146/annurev.ne.02.030179.002311

Kupfermann, I. (1974). Feeding behavior in Aplysia: a simple system for the study of motivation. Behav. Biol. 10, 1-26. doi: 10.1016/s0091-6773(74)91644-7

Kupfermann, I., and Weiss, K. R. (1982). Activity of an identified serotonergic neuron in free moving Aplysia correlates with behavioral arousal. Brain Res. 241, 334-337. doi: 10.1016/00068993(82)91072-1

Lei, H., Christensen, T. A., \& Hildebrand, J. G. (2002). Local inhibition modulates odor-evoked synchronization of glomerulus-specific output neurons. Nature neuroscience, 5(6), 557.

Lei, H., Reisenman, C. E., Wilson, C. H., Gabbur, P., \& Hildebrand, J. G. (2011). Spiking patterns and their functional implications in the antennal lobe of the tobacco hornworm Manduca sexta. PLoS One, 6(8), e23382.

Linn, C. E., and Roelofs, W. L. (1986). Modulatory effects of octopamine and serotonin on male sensitivity and periodicity of response to sex-pheromone in the cabbage-looper moth, trichoplusia-ni. Arch. Insect Biochem. Physiol. 3, 161-171. doi: 10.1002/arch.940030206

Linster, C., and Cleland, T. A. (2016). Neuromodulation of olfactory transformations. Curr. Opin. Neurobiol. 40, 170-177. doi: 10.1016/j.conb.2016.07.006

Lizbinski, K. M., Marsat, G., \& Dacks, A. M. (2018). Systematic Analysis of Transmitter Coexpression Reveals Organizing Principles of Local Interneuron Heterogeneity. eNeuro, 5(5).

Lizbinski, K. M., Metheny, J. D., Bradley, S. P., Kesari, A., and Dacks, A. M. (2016). The anatomical

basis for modulatory convergence in the antennal lobe of Manduca sexta. J. Comp. Neurol. 524, 18591875. doi: $10.1002 /$ cne. 23926 
MacLeod, K., and Laurent, G. (1996). Distinct mechanisms for synchronization and temporal patterning of odor-encoding neural assemblies. Science 274, 976-979. doi: 10.1126/science.274.5289.976

Mandairon, N., Ferretti, C. J., Stack, C. M., Rubin, D. B., Cleland, T. A., and Linster, C. (2006).

Cholinergic modulation in the olfactory bulb influences spontaneous olfactory discrimination in adult rats. Eur. J. Neurosci. 24, 3234-3244. doi: 10.1111/j.1460-9568.2006.05212.x

Marder, E. (2012). Neuromodulation of neuronal circuits: back to the future. Neuron, 76(1), 1-11

Morgan, P. T., Perrins, R., Lloyd, P. E., and Weiss, K. R. (2000). Intrinsic and extrinsic modulation of a single central pattern generating circuit. J. Neurophysiol. 84, 1186-1193. doi:

10.1152/jn.2000.84.3.1186

Martin, J. P., Beyerlein, A., Dacks, A. M., Reisenman, C. E., Riffell, J. A., Lei, H., \& Hildebrand, J. G.

(2011). The neurobiology of insect olfaction: sensory processing in a comparative context. Progress in neurobiology, 95(3), 427-447

Matsutani, S., and Yamamoto, N. (2008). Centrifugal innervation of the mammalian olfactory bulb. Anat. Sci. Int. 83, 218-227. doi: 10.1111/j.1447-073X.2007.00223.x

McLean, J. H., and Shipley, M. T. (1987). Serotonergic afferents to the rat olfactory bulb: I. Origins and laminar specificity of serotonergic inputs in the adult rat. J. Neurosci. 7, 3016-3028.

Mercer, A. R., Hayashi, J. H., and Hildebrand, J. G. (1995). Modulatory effects of 5-hydroxytryptamine

on voltage-activated currents in cultured antennal lobe neurones of the sphinx moth Manduca sexta. J. Exp. Biol. 198, 613-627.

Mercer, A. R., Kloppenburg, P., and Hildebrand, J. G. (1996). Serotonin-induced changes in the excitability of cultured antennal-lobe neurons of the sphinx moth Manduca sexta. J. Comp. Physiol. A 178, 21-31. doi: 10.1007/bf00189587

Mesce, K. A., Crisp, K. M., \& Gilchrist, L. S. (2001). Mixtures of octopamine and serotonin have nonadditive effects on the CNS of the medicinal leech. Journal of neurophysiology, 85(5), 20392046.

Murphy, G. J., Glickfeld, L. L., Balsen, Z., and Isaacson, J. S. (2004). Sensory neuron signaling to the brain: properties of transmitter release from olfactory nerve terminals. J. Neurosci. 24, 3023-3030. doi: 10.1523/JNEUROSCI.5745-03.2004

Mustard, J. A., Beggs, K. T., \& Mercer, A. R. (2005). Molecular biology of the invertebrate dopamine receptors. Archives of Insect Biochemistry and Physiology: Published in Collaboration with the Entomological Society of America, 59(3), 103-117.

Nadim, F., \& Bucher, D. (2014). Neuromodulation of neurons and synapses. Current opinion in neurobiology, 29, 48-56.

Nagayama, S., Homma, R., and Imamura, F. (2014). Neuronal organization of olfactory bulb circuits. Front. Neural Circuits 8:98. doi: 10.3389/fncir.2014.00098

Nässel, D. R., \& Homberg, U. (2006). Neuropeptides in interneurons of the insect brain. Cell and tissue research, 326(1), 1 . 
Nässel, D. R. (2018). Substrates for neuronal cotransmission with neuropeptides and small molecule neurotransmitters in Drosophila. Frontiers in cellular neuroscience, 12, 83.

Nusbaum, M. P., Blitz, D. M., Swensen, A. M., Wood, D., \& Marder, E. (2001). The roles of cotransmission in neural network modulation. Trends in neurosciences, 24(3), 146-154.

Nusbaum, M. P., Blitz, D. M., \& Marder, E. (2017). Functional consequences of neuropeptide and smallmolecule co-transmission. Nature Reviews Neuroscience, 18(7), 389.

Olsen, S. R., Bhandawat, V., \& Wilson, R. I. (2007). Excitatory interactions between olfactory processing channels in the Drosophila antennal lobe. Neuron, 54(1), 89-103.

Olsen, S. R., and Wilson, R. I. (2008). Lateral presynaptic inhibition mediates gain control in an olfactory circuit. Nature 452, 956-960. doi: 10.1038/nature06864

Olsen, S. R., Bhandawat, V., and Wilson, R. I. (2010). Divisive normalization in olfactory population codes. Neuron 66, 287-299. doi: 10.1016/j.neuron.2010.04.009

Owald, D., and Waddell, S. (2015). Olfactory learning skews mushroom body output pathways to steer behavioral choice in Drosophila. Curr. Opin. Neurobiol. 35, 178-184. doi:

10.1016/j.conb.2015.10.002

Park, S. P., Lopez-Rodriguez, F., Wilson, C. L., Maidment, N., Matsumoto, Y., and Engel, J. Jr. (1999). In vivo microdialysis measures of extracellular serotonin in the rat hippocampus during sleep- $\quad$ wakefulness. Brain Res. 833, 291-296. doi: 10.1016/s0006-8993(99)01511-5

Perk, C. G., \& Mercer, A. R. (2006). Dopamine modulation of honey bee (Apis mellifera) antennal-lobe neurons. Journal of neurophysiology, 95(2), 1147-1157.

Proekt, A., and Weiss, K. R. (2003). Convergent mechanisms mediate preparatory states and repetition priming in the feeding network of Aplysia. J. Neurosci. 23, 4029-4033.

Rehder, V., Bicker, G., and Hammer, M. (1987). Serotonin-immunoreactive neurons in the antennal lobes and suboesophageal ganglion of the honeybee. Cell Tissue Res. 247, 59-66. doi: $10.1007 / \mathrm{bf00216547}$

Reisenman, C. E., Dacks, A. M., and Hildebrand, J. G. (2011). Local interneuron diversity in the primary olfactory center of the moth Manduca sexta. J. Comp. Physiol. A Neuroethol. Sens. Neural Behav. Physiol. 197, 653-665. doi: 10.1007/s00359-011-0625-x

Ren, J., Friedmann, D., Xiong, J., Liu, C. D., Ferguson, B. R., Weerakkody, T., ... \& Weissbourd, B. (2018). Anatomically Defined and Functionally Distinct Dorsal Raphe Serotonin Sub-systems. Cell.

Riffell, J. A., Lei, H., Abrell, L., \& Hildebrand, J. G. (2013). Neural basis of a pollinator's buffet: olfactory specialization and learning in Manduca sexta. Science, 339(6116), 200-204.

Riffell, J. A., Lei, H., \& Hildebrand, J. G. (2009). Neural correlates of behavior in the moth Manduca sexta in response to complex odors. Proceedings of the National Academy of Sciences, pnas0910592106.

Rosen, S. C., Weiss, K. R., Goldstein, R. S., and Kupfermann, I. (1989). The role of a modulatory neuron in feeding and satiation in Aplysia: effects of lesioning of the serotonergic metacerebral cells. $J$. Neurosci. 9, 1562-1578. 
Root, C. M., Masuyama, K., Green, D. S., Enell, L. E., Nässel, D. R., Lee, C. H., et al. (2008). A presynaptic gain control mechanism fine-tunes olfactory behavior. Neuron 59, 311-321. doi: 10.1016/j.neuron.2008.07.003

Root, C. M., Ko, K. I., Jafari, A., and Wang, J. W. (2011). Presynaptic facilitation by neuropeptide signaling mediates odor-driven food search. Cell 145, 133-144. doi: 10.1016/j.cell.2011.02.008

Roy, B., Singh, A. P., Shetty, C., Chaudhary, V., North, A., Landgraf, M., et al. (2007). Metamorphosis of an identified serotonergic neuron in the Drosophila olfactory system. Neural Dev. 2:20. doi: $10.1186 / 1749-8104-2-20$

Sachse, S., and Beshel, J. (2016). The good, the bad, and the hungry: how the central brain codes odor valence to facilitate food approach in Drosophila. Curr. Opin. Neurobiol. 40, 53-58. doi: 10.1016/j.conb.2016.06.012

Saudou, F., Boschert, U., Amlaiky, N., Plassat, J. L., \& Hen, R. (1992). A family of Drosophila serotonin receptors with distinct intracellular signalling properties and expression patterns. The EMBO journal, 11(1), 7-17.

Salecker, I., and Distler, P. (1990). Serotonin-immunoreactive neurons in the antennal lobes of the American cockroach Periplaneta americana: light- and electron-microscopic observations. Histochemistry 94, 463-473. doi: 10.1007/bf00272608

Salio, C., Lossi, L., Ferrini, F., \& Merighi, A. (2006). Neuropeptides as synaptic transmitters. Cell and tissue research, 326(2), 583-598.

Saraswati, S., Adolfsen, B., \& Littleton, J. T. (2007). Characterization of the role of the Synaptotagmin family as calcium sensors in facilitation and asynchronous neurotransmitter release. Proceedings of the National Academy of Sciences, 104(35), 14122-14127.

Schachtner, J., Trosowski, B., D'Hanis, W., Stubner, S., \& Homberg, U. (2004). Development and steroid regulation of RFamide immunoreactivity in antennal-lobe neurons of the sphinx moth Manduca sexta. Journal of experimental biology, 207(14), 2389-2400.

Schoppa, N. E. (2006). Synchronization of olfactory bulb mitral cells by precisely timed inhibitory inputs. Neuron, 49(2), 271-283.

Schroll, C., Riemensperger, T., Bucher, D., Ehmer, J., Völler, T., Erbguth, K., ... \& Fiala, A. (2006).

Light- induced activation of distinct modulatory neurons triggers appetitive or aversive learning in Drosophila larvae. Current biology, 16(17), 1741-1747.

Schwaerzel, M., Monastirioti, M., Scholz, H., Friggi-Grelin, F., Birman, S., \& Heisenberg, M. (2003). Dopamine and octopamine differentiate between aversive and appetitive olfactory memories in Drosophila. Journal of Neuroscience, 23(33), 10495-10502.

Schultzhaus, J. N., Saleem, S., Iftikhar, H., and Carney, G. E. (2017). The role of the Drosophila lateral horn in olfactory information processing and behavioral response. J. Insect Physiol. 98, 29-37. doi: 10.1016/j.jinsphys.2016.11.007

Seki, Y., and Kanzaki, R. (2008). Comprehensive morphological identification and GABA immunocytochemistry of antennal lobe local interneurons in Bombyx mori. J. Comp. Neurol. 506, 93-107. doi: 10.1002/cne.21528 
Seki, Y., Rybak, J., Wicher, D., Sachse, S., and Hansson, B. S. (2010). Physiological and morphological characterization of local interneurons in the Drosophila antennal lobe. J. Neurophysiol. 104, 1007-1019. doi: 10.1152/jn.00249.2010

Siju, K. P., Reifenrath, A., Scheiblich, H., Neupert, S., Predel, R., Hansson, B. S., et al. (2014). Neuropeptides in the antennal lobe of the yellow fever mosquito, Aedes aegypti. J. Comp. Neurol. 522, 592-608. doi: 10.1002/cne.23434

Singh, A. P., Das, R. N., Rao, G., Aggarwal, A., Diegelmann, S., Evers, J. F., et al. (2013). Sensory neuron- derived eph regulates glomerular arbors and modulatory function of a central serotonergic neuron. PLoS Genet. 9:e1003452. doi: 10.1371/journal.pgen.1003452

Sinakevitch, I., Niwa, M., and Strausfeld, N. J. (2005). Octopamine-like immunoreactivity in the honey bee and cockroach: comparable organization in the brain and subesophageal ganglion. J. Comp. Neurol. 488, 233-254. doi: 10.1002/cne.20572

Sinakevitch, I., and Strausfeld, N. J. (2006). Comparison of octopamine-like immunoreactivity in the brains of the fruit fly and blow fly. J. Comp. Neurol. 494, 460-475. doi: 10.1002/cne.20799

Sizemore, T. R., and Dacks, A. M. (2016). Serotonergic modulation differentially targets distinct network elements within the antennal lobe of Drosophila melanogaster. Sci. Rep. 6:37119. doi: $10.1038 /$ srep37119

Steinfeld, R., Herb, J. T., Sprengel, R., Schaefer, A. T., and Fukunaga, I. (2015). Divergent innervation of the olfactory bulb by distinct raphe nuclei. J. Comp. Neurol. 523, 805-813. doi: $10.1002 /$ cne. 23713

Sun, X. J., Tolbert, L. P., and Hildebrand, J. G. (1993). Ramification pattern and ultrastructural characteristics of the serotonin-immunoreactive neuron in the antennal lobe of the moth Manduca sexta: a laser scanning confocal and electron microscopic study. J. Comp. Neurol. 338, 5-16. doi: 10.1002/cne.903380103

Tedjakumala, S. R., Rouquette, J., Boizeau, M. L., Mesce, K. A., Hotier, L., Massou, I., et al. (2017). A tyrosine-hydroxylase characterization of dopaminergic neurons in the honey bee brain. Front. Syst. Neurosci. 11:47. doi: 10.3389/fnsys.2017.00047

Thirumalai, V., \& Marder, E. (2002). Colocalized neuropeptides activate a central pattern generator by acting on different circuit targets. Journal of Neuroscience, 22(5), 1874-1882.

Tierney, A. J. (2018). Invertebrate serotonin receptors: a molecular perspective on classification and pharmacology. Journal of Experimental Biology, 221(19), jeb184838.

Tripathy, S., Staudacher, E. M., Peters, O., Kalwar, F., Hatfield, M., \& Daly, K. (2010). Odors pulsed at wing beat frequencies are tracked by primary olfactory networks and enhance odor detection. Frontiers in cellular neuroscience, 4,1 .

Tritsch, N. X., Granger, A. J., \& Sabatini, B. L. (2016). Mechanisms and functions of GABA corelease. Nature Reviews Neuroscience, 17(3), 139.

Trulson, M. E., and Jacobs, B. L. (1979). Raphe unit activity in freely moving cats: correlation with level of $\quad$ behavioral arousal. Brain Res.163, 135-150. doi: 10.1016/0006-8993(79)90157-4 
Unoki, S., Matsumoto, Y., \& Mizunami, M. (2006). Roles of octopaminergic and dopaminergic neurons

in mediating reward and punishment signals in insect visual learning. European Journal of Neuroscience, 24(7), 2031-2038.

Utz, S., Huetteroth, W., Wegener, C., Kahnt, J., Predel, R., \& Schachtner, J. (2007). Direct peptide profiling of lateral cell groups of the antennal lobes of Manduca sexta reveals specific composition and changes in neuropeptide expression during development. Developmental neurobiology, 67(6), 764-777.

Utz, S., Huetteroth, W., Vömel, M., and Schachtner, J. (2008). Mas-allatotropin in the developing antennal lobe of the sphinx moth Manduca sexta: distribution, time course, developmental regulation, and colocalization with other neuropeptides. Dev. Neurobiol. 68, 123-142. doi: 10.1002/dneu.20579

Utz, S., Huetteroth, W., Vömel, M., \& Schachtner, J. (2008). Mas-allatotropin in the developing antennal lobe of the sphinx moth Manduca sexta: distribution, time course, developmental regulation, and colocalization with other neuropeptides. Developmental neurobiology, 68(1), 123-142.

Vinauger, C., Lahondère, C., Wolff, G. H., Locke, L. T., Liaw, J. E., Parrish, J. Z., ... \& Riffell, J. A. (2018). Modulation of host learning in Aedes aegypti mosquitoes. Current Biology, 28(3), 333-344.

Vosshall, L. B., Amrein, H., Morozov, P. S., Rzhetsky, A., and Axel, R. (1999). A spatial map of

olfactory receptor expression in the Drosophila antenna. Cell 96, 725-736. doi: 10.1016/s00928674(00)80582-6

Vosshall, L. B. (2000). Olfaction in Drosophila. Curr. Opin. Neurobiol. 10, 498-503. doi:

10.1016/S0959-4 388(00)00111-2

Waldrop, B., Christensen, T. A., and Hildebrand, J. G. (1987). GABA-mediated synaptic inhibition of projection neurons in the antennal lobes of the sphinx moth, Manduca sexta. J. Comp. Physiol. A 161, 23-32. doi: 10.1007/bf00609452

Wang, J. W. (2012). Presynaptic modulation of early olfactory processing in Drosophila. Dev. Neurobiol. 72, 87-99. doi: 10.1002/dneu.20936

Wasserman, S., Salomon, A., and Frye, M. A. (2013). Drosophila tracks carbon dioxide in flight. Curr. Biol. 23, 301-306. doi: 10.1016/j.cub.2012.12.03

Wegerhoff, R. (1999). GABA and serotonin immunoreactivity during postembryonic brain development in the beetle Tenebrio molitor. Microsc. Res. Tech. 45, 154-164. doi: 10.1002/(sici)10970029(19990501)45:3<154::aid-jemt3>3.0.co;2-5

Wilson, R. I., Turner, G. C., and Laurent, G. (2004). Transformation of olfactory representations in the Drosophila antennal lobe. Science303, 366-370. doi: 10.1126/science.1090782

Wilson, R. I., and Laurent, G. (2005). Role of GABAergic inhibition in shaping odor-evoked spatiotemporal patterns in the Drosophilaantennal lobe. J. Neurosci. 25, 9069-9079. doi: 10.1523/jneurosci.2070-05.2005

Wilson, R. I. (2013). Early olfactory processing in Drosophila: mechanisms and principles. Annual Review of Neuroscience, 36, 217-241. 
Won, M. H., Ohno, T., Suh, J. G., Lee, J. C., Jo, S. M., Oh, Y. S., et al. (1998). Serotonergic neurons are present and innervate blood vessels in the olfactory bulb of the laboratory shrew, Suncus murinus. Neurosci. Lett. 243, 53-56. doi: 10.1016/s0304-3940(98)00084-6

Xu, L., He, J., Kaiser, A., Gräber, N., Schläger, L., Ritze, Y., et al. (2016). A single pair of serotonergic neurons counteracts serotonergic inhibition of ethanol attraction in Drosophila. PLoS One 11:e0167518. doi: 10.1371/journal.pone.0167518

Yapici, N., Kim, Y. J., Ribeiro, C., \& Dickson, B. J. (2008). A receptor that mediates the post-mating switch in Drosophila reproductive behaviour. Nature, 451(7174), 33.

Zars, T. (2000). Behavioral functions of the insect mushroom bodies. Curr. Opin. Neurobiol. 10, 790 795. doi: 10.1016/s0959-4388(00)00147-1

Zhang, X., and Gaudry, Q. (2016). Functional integration of a serotonergic neuron in the Drosophila antennal lobe. Elife 5:e16836. doi: 10.7554/eLife.16836 


\section{CHAPTER 2}

The Anatomical Basis for Modulatory Integration of serotonin and dopamine in the Antennal Lobe 


\section{Summary:}

Within a network, functionally distinct neuron types often innervate different anatomical regions, creating a physical map within the brain that corresponds to particular stimulus parameters. The idea of functional mapping of neurons based on location within a network is common across many brain regions including the visual cortex (Hubel and Wiesel, 1965), cochlea (Clopton et al., 1974), and auditory cortex (Knudsen and Konishi, 1978; Suga et al., 1979). Similar to functional mapping based on the stimulus neurons respond to, input and output neurons can also occupy defined regions within a particular brain region. The olfactory system is known for its highly stereotyped anatomy, wherein the input, output and local processing neurons occupy defined regions in anatomical space (Shipley and Ennis, 1996). This organization not only allows for "odotopic" mapping across individual functional units that respond to specific odors called glomeruli, but also allows modulatory neurons to segregate (or integrate) their influence based on the region of the network they project to. For example, within the olfactory bulb of vertebrates, the serotonergic Raphe nuclei preferentially innervate the glomerular layer that contains mostly juxtaglomerular neurons (the local processing neurons) and olfactory sensory neurons (the input neurons) (Pinching and Powell, 1971; McLean and Shipley, 1987; Shipley and Ennis, 1996; Gomez et al., 2005; Steinfeld et al., 2015; Suzuki et al., 2015; Muzurelle et al., 2016). Modulatory neurons have also been shown to differentially innervate even more specific regions within the olfactory system, such as glomerular specific innervation (Coates et al., 2017; Gómez et al., 2005). This allows for odor-specific or cell-type specific targeting of modulation. However, most, if not all, sensory networks receive multiple neuromodulatory inputs and the mechanisms by which sensory networks integrate or segregate multiple modulatory inputs are not well understood. Here, I take advantage of the anatomically tractable olfactory system of Manduca sexta to determine the anatomical basis of the integration of two neuromodulators, serotonin and dopamine.

The first synaptic neuropil of the insect olfactory system, the antennal lobe (AL), is a sensory network comprised of three primary neuron types. Odor-responsive olfactory receptor neurons (ORNs) on the antennae project centrally to the AL where they terminate in $\sim 63$ substructures called glomeruli (Rospars and Hildebrand, 2000; Huetteroth and Schachtner, 2005). Within each glomerulus, ORNs provide input to projection neurons (PNs) and the transfer of information from ORNs to PNs is refined by local interneurons (LNs) that interconnect each 
glomerulus. Glomeruli surround a "coarse neuropil" containing the thicker processes of LNs and PNs in which there is little, if any, synaptic interaction (reviewed in Ache and Young, 2005). The orientation of glomeruli is considered with respect to the coarse neuropil, with the proximal half of the glomerulus facing the coarse neuropil and the distal half facing away from the coarse neuropil. PNs and LNs innervate glomeruli from the proximal end whereas ORNs innervate glomeruli from the distal end. Thus, principal neurons types of the AL have defined anatomical boundaries within glomeruli. In addition to these three primary neuron types, a number of extrinsic neurons project to the AL including two sets of widely projecting neurons releasing 5HT (Kent et al., 1987) and DA (Dacks et al., 2012). The contralaterally projecting serotoninimmunoreactive deutocerebral neuron (CSD) (Kent et al., 1987; Dacks et al., 2006) and dopaminergic arching neurons (DAAR) (Dacks et al., 2012) project widely throughout the brain and innervate every glomerulus of the AL.

However, it is unknown whether each modulator differs in their relative distribution to principal neurons types of the AL, i.e. ORNs, LNs and PNs. Using immunocytochemistry, dye fills, and quantitative analysis of each neuron types' distribution within a glomerulus, I determined 1) the distribution of each modulatory neuron within olfactory glomeruli and 2) the functional glomerular zones each modulatory neuron innervates. Specifically, I characterized the innervation patterns of both 5-HT and tyrosine hydroxylase immunoreactive processes (TH-ir, the rate-limiting enzyme for dopamine synthesis) relative to each other, olfactory receptor neurons (ORNs), projection neurons (PNs) and several subsets of local interneurons (LNs). I found that 5-HT-ir had nearly complete overlap with PNs and LNs, yet no overlap with ORNs, suggesting that 5-HT may modulate PNs and LNs directly but not ORNs. TH-ir overlapped with PNs, LNs and ORNs suggesting that dopamine has the potential to modulate all three principal olfactory cell types. Furthermore, the branching density of each neuromodulator differed with 5HT exhibiting denser arborizations and TH-ir processes being sparser. Our results suggest that 5HT and DA extrinsic neurons target partially overlapping glomerular regions, yet DA extends further into the region occupied by ORNs (Lizbinski et al., 2016). Ultimately, I describe a detailed map of 5-HT and DA distribution with relation to populations of AL neurons, providing the anatomical basis for integration of two extrinsic modulators on olfactory processing. 


\section{Works Cited:}

Ache, B. W., \& Young, J. M. (2005). Olfaction: diverse species, conserved principles. Neuron, 48(3), $417-430$.

Clopton, B. M., Winfield, J. A., \& Flammino, F. J. (1974). Tonotopic organization: review and analysis. Brain research, 76(1), 1-20.

Dacks, A. M., Christensen, T. A., \& Hildebrand, J. G. (2006). Phylogeny of a serotonin-immunoreactive neuron in the primary olfactory center of the insect brain. Journal of Comparative Neurology, 498(6), 727-746.

Dacks, A. M., Riffell, J. A., Martin, J. P., Gage, S. L., \& Nighorn, A. J. (2012). Olfactory modulation by dopamine in the context of aversive learning. Journal of neurophysiology, 108(2), 539-550.

Gómez, C., Briñón, J. G., Barbado, M. V., Weruaga, E., Valero, J., and Alonso, J. R. (2005).

Heterogeneous targeting of centrifugal inputs to the glomerular layer of the main olfactory bulb. J.

Chem. Neuroanat. 29, 238-254.

Hubel, D. H., \& Wiesel, T. N. (1965). Receptive fields and functional architecture in two nonstriate visual areas (18 and 19) of the cat. Journal of neurophysiology, 28(2), 229-289.

Huetteroth, W., \& Schachtner, J. (2005). Standard three-dimensional glomeruli of the Manduca sexta antennal lobe: a tool to study both developmental and adult neuronal plasticity. Cell and tissue research, 319(3), 513-524.

Kent, K. S., Hoskins, S. G., \& Hildebrand, J. G. (1987). A novel serotonin-immunoreactive neuron in the antennal lobe of the sphinx moth Manduca sexta persists throughout postembryonic life. Journal of neurobiology, 18(5), 451-465.

Knudsen, E. I., \& Konishi, M. (1978). Space and frequency are represented separately in auditory midbrain of the owl. Journal of Neurophysiology, 41(4), 870-884.

Lizbinski, K. M., Metheny, J. D., Bradley, S. P., Kesari, A., \& Dacks, A. M. (2016). The anatomical basis

for modulatory convergence in the antennal lobe of Manduca sexta. Journal of Comparative Neurology, 524(9), 1859-1875.

McLean, J. H., \& Shipley, M. T. (1987). Serotonergic afferents to the rat olfactory bulb: I. Origins and laminar specificity of serotonergic inputs in the adult rat. Journal of Neuroscience, 7(10), 30163028Pinching, A. J., \& Powell, T. P. S. (1971). The neuropil of the glomeruli of the olfactory bulb. Journal of cell science, 9(2), 347-377.

Muzerelle, A., Scotto-Lomassese, S., Bernard, J. F., Soiza-Reilly, M., \& Gaspar, P. (2016). Conditional anterograde tracing reveals distinct targeting of individual serotonin cell groups (B5-B9) to the forebrain and brainstem. Brain Structure and Function, 221(1), 535-561.

Rospars, J. P., \& Hildebrand, J. G. (2000). Sexually dimorphic and isomorphic glomeruli in the antennal lobes of the sphinx moth Manduca sexta. Chemical Senses, 25(2), 119-129.

Suga, N., \& O'Neill, W. E. (1979). Neural axis representing target range in the auditory cortex of the mustache bat. Science, 206(4416), 351-353. 
Shipley, M. T., \& Ennis, M. (1996). Functional organization of olfactory system. Journal of neurobiology, 30(1), 123-176.

Steinfeld, R., Herb, J. T., Sprengel, R., Schaefer, A. T., \& Fukunaga, I. (2015). Divergent innervation of the olfactory bulb by distinct raphe nuclei. Journal of Comparative Neurology, 523(5), 805-813. 
(this chapter is taken directly from my publication "Lizbinski, K. M., Metheny, J. D., Bradley, S. P., Kesari, A., \& Dacks, A. M. (2016). The anatomical basis for modulatory convergence in the antennal lobe of Manduca sexta. Journal of Comparative Neurology, 524(9), 1859-1875 '.)

\section{Abstract:}

The release of neuromodulators by widely projecting neurons often allows sensory systems to alter how they process information based on the physiological state of an animal. Neuromodulators alter network function by changing the biophysical properties of individual neurons and the synaptic efficacy with which individual neurons communicate. However, most, if not all, sensory networks receive multiple neuromodulatory inputs and the mechanisms by which sensory networks integrate multiple modulatory inputs are not well understood. Here, we characterized the relative glomerular distribution of two extrinsic neuromodulators associated with distinct physiological states, serotonin (5-HT) and dopamine (DA), in the antennal lobe (AL) of the moth Manduca sexta. Using immunocytochemistry and mass dye fills, we characterized the innervation patterns of both 5-HT and tyrosine hydroxylase immunoreactive processes (TH-ir) relative to each other, olfactory receptor neurons (ORNs), projection neurons (PNs) and several subsets of local interneurons (LNs). 5-HT-ir had nearly complete overlap with PNs and LN, yet no overlap with ORNs, suggesting that 5-HT may modulate PNs and LNs directly but not ORNs. TH-ir overlapped with PNs, LNs and ORNs suggesting that dopamine has the potential to modulate all three cell types. Furthermore, the branching density of each neuromodulator differed with 5-HT exhibiting denser arborizations and TH-ir processes being more sparse. Our results suggest that 5-HT and DA extrinsic neurons target partially overlapping glomerular regions, yet DA extends further into the region occupied by ORNs.

\section{Introduction:}

The nervous system adjusts the response properties of individual neural networks based on the current physiological state in order to optimize network function (Nusbaum and Marder, 1989; Fontanini and Katz, 2006; Hurley and Hall, 2011; Komuniecki et al., 2014; Su and Wang, 2014). This is often accomplished by widely projecting neurons that respond to changes in the physiological state and release neuromodulators that alter the synaptic efficacy or biophysical properties of individual neurons (Kupfermann, 1979; Kaczmarek and Levitan, 1987; Katz, 1999) with single neuromodulators potentially having wide-ranging and diverse effects on a network. 
Although a network is constrained by its anatomical layout, modulation of synaptic and cellular mechanisms influences its functional connectivity (Getting, 1989). Thus, the process of neuromodulation expands the dynamic range of a network by adjusting the response properties and interactions of a constant set of neurons. Every neural network is influenced by a dynamic cocktail of multiple neuromodulators (Brezina, 2010; Bargmann, 2012; Marder, 2012; Nusbaum and Blitz, 2012) and single neurons integrate their effects. Overall, many factors, including receptor type, second messenger system, and connectivity allow neuromodulation to provide a remarkable degree of control over network parameters. While the convergence of multiple neuromodulators within a network has been studied extensively in motor systems, the consequences of this convergence in sensory systems have not received as much attention.

To understand the potential impact of modulatory convergence within a sensory system, we sought to first characterize the relative anatomical distribution of multiple neuromodulators within a single sensory system. The physiological effects of serotonin (5-HT) and dopamine (DA) have been well-characterized individually in the olfactory system of Manduca sexta thus making this model system well-positioned to study their combined effects. The first synaptic neuropil of the insect olfactory system, the antennal lobe (AL), is a network comprised of three primary neuron types. Odor-responsive olfactory receptor neurons (ORNs) on the antennae project centrally to the AL where they terminate in $~ 63$ sub-structures called glomeruli (Rospars and Hildebrand, 2000; Huetteroth and Schachtner, 2005). Within each glomerulus, ORNs provide input to projection neurons (PNs) and the transfer of information from ORNs to PNs is refined by local interneurons (LNs) that interconnect each glomerulus. The glomeruli surround a "coarse neuropil" containing the thicker processes of LNs and PNs in which there are little, if any, sites of synaptic interaction (reviewed in Ache and Young, 2005). The orientation of glomeruli is considered with respect to the coarse neuropil, with the proximal half of the glomerulus facing the coarse neuropil and the distal half of the glomerulus facing away from the coarse neuropil. PNs and LNs innervate glomeruli from the proximal end whereas ORNs innervate glomeruli from the distal end.

In addition to these three primary neuron types, a variety of extrinsic neurons project to the AL including two sets of widely projecting neurons releasing 5-HT (Kent et al., 1987) and DA (Dacks et al., 2012). Both modulators enhance neuronal excitability and odor evoked responses in isolation (Kloppenburg and Hildebrand, 1995; Mercer et al., 1995; Mercer et al., 
1996; Kloppenburg et al., 1999; Kloppenburg and Heinbockel, 2000; Dacks et al., 2008; Dacks et al., 2012), but their anatomical convergence within individual glomeruli is unknown. The goal of this study was to determine the relative glomerular distribution of 5-HT and DA with relation to the primary neuron types in the AL of Manduca sexta. We found that the relative distribution of the processes of both 5-HT and DA neurons suggests that both modulators are more likely to have convergent effects upon LNs and PNs, while DA neurons are more likely to influence ORNs.

\section{Materials and Methods:}

Animals: Manduca sexta (hereafter referred to as Manduca) was raised at West Virginia University on a 16:8 reverse light:dark cycle in the laboratory of Dr. Kevin Daly as previously described (Bell and Joachim, 1976; Daly et al., 2013). Equal numbers of males and females were used and a minimum of 6 moths were used for each protocol.

Immunocytochemistry: Table 1 provides the complete list of antibodies used in this study. Brains were dissected in physiological saline (Christensen and Hildebrand, 1987), fixed in 4\% paraformaldehyde overnight at $4^{\circ} \mathrm{C}$, run through a dehydrating ethanol series and then embedded in 5\% agarose to be sectioned at 100um using a Leica VT 1000S vibratome with the exception of tissue labeled for GABA (75 um) and BRP, Manduca tyrosine hydroxylase MsTH and 5-HT triple label (250 um). Sections were washed in PBS with 1\% Triton X-100 (PBST), blocked in PBST and 2\% IgG free BSA (Jackson Immunoresearch; Cat\#001-000-161) and then incubated in blocking solution with $5 \mathrm{mM}$ sodium azide and primary antibody. Incubation period and temperature, as well as dilution, were primary antibody specific (see Antibody Characterization section and Table 1). Sections were washed with PBST, blocked as above and fluorescent secondary antibodies against the primary antibody host species were applied overnight at a dilution of 1:1000 at room temperature with the exception of the anti-BRP antibody which was incubated at $4^{\circ} \mathrm{C}$. The fluorescent secondary antibodies used (all from Life Technologies) were donkey $\alpha$-rabbit Alexa 488 (Cat\# A-21206), donkey $\alpha$-mouse Alexa 546 (Cat\# A10036) and donkey $\alpha$-goat Alexa 633 (Cat\# A-21082). Sections were then briefly washed with PBS (pH 6.9), PBST, and a series of ascending glycerol washes and then mounted on slides with Vectashield (Vector Laboratories; Cat\#H-1000). For peptide pre-adsorptions, all custom lyophilized peptides 
were synthesized by GenScript. To obtain the Manduca specific peptide sequences, we performed a forward protein BLAST analysis of the Drosophila pre-propeptide sequence for TKK and MIP in the Manduca genome (Agricultural Pest Genomics Resource Database: www.agripestbase.org). Using the top matches from the Manduca genome, we then reverse blasted the sequence from the Manduca genome into the Drosophila genome to ensure that our result returned the lowest $E$ values, indicating that our sequence shared close homology to the Drosophila peptide sequence that we used as a reference. The peptide sequences used for preadsorption controls had high sequence identity with Drosophila TKK $_{1}$ (RAPMGFMGVR in Manduca) and MIP $\mathrm{VI}_{\mathrm{I}}$ (AWSALHGAWA in Manduca).

Dye fills: Antennal nerve fills were performed as previously described (Dacks et al., 2010) using Dextran, Texas Red®, 3000 MW, Neutral (LifeTechnologies, Cat\# D-3329). Brains were dissected after 24 hours for subsequent immunocytochemistry. For mushroom body dye fills, moths were waxed into tubes with their heads tilted dorsally, a window cut in the head capsule to expose the mushroom bodies and Dextran, Texas Red was injected into each mushroom body using a pulled glass capillary tube. The cuticle was placed back over the window, the window sealed with petroleum jelly and brains were dissected out for subsequent immunocytochemistry 48-72 hours later to allow diffusion of dye. Moths were fed high concentration sugar water before the dye injection and on each subsequent day to increase survival rates.

\section{Antibody Characterization}

Bruchpilot (BRP) - The BRP antiserum (nc-82: Developmental Studies Hybridoma Bank) was raised in mouse against the Drosophila melanogaster protein "Bruchpilot" (Hofbauer, 1991) which is a homologue of the presynaptic active zone protein ELKS/CAST and is required for functional synapses in the nervous system of Drosophila (Wagh et al., 2006). This antibody is used in many insect species to delineate brain neuropil allowing visualization of brain regions and their individual compartments (Ito et al., 2014). We used the BRP antibody to delineate glomerular boundaries within the AL of Manduca and although the purpose of using this antibody was not to study the distribution of the Manduca homologue of ELKS/CAST, we performed a Western blot to verify that the antibody labeled a single protein at the predicted 
height for the Manduca homologue of ELKS/CAST. Using the Manduca genome (Agricultural Pest Genomics Resource Database: www.agripestbase.org) we performed a forward protein BLAST analysis of the Drosophila Bruchpilot amino acid sequence. The top match from the Manduca genome had an e-value of 0.0 and a predicted molecular weight of $234.63 \mathrm{kDa}$. We then reverse blasted the sequence from the Manduca genome into the Drosophila genome and the first 7 matches were Bruchpilot isoforms, all of which had e-values of 0.0. The next highest match from the Drosophila genome had an e-value of 0.71, indicating that the sequence pulled from the Manduca genome had a very high sequence homology to Drosophila Bruchpilot and almost no homology to other proteins. To ensure that there were not two potential predicted protein sequences from the Manduca genome with high sequence homology to Drosophila Bruchpilot, we took the amino acid sequence from the Manduca genome with the second highest e-value for the Drosophila Bruchpilot (e-value $=7 \mathrm{e}-38$ ) and ran a BLAST analysis of that amino acid sequence in the Drosophila genome. The BLAST analysis resulted in an e-value of 0.0 for the Drosophila protein Stretchin-MLCK (Champagne et al., 2000), indicating that the Manduca protein with the next closest sequence similarity to Drosophila Bruchpilot was not a Bruchpilot homologue.

We performed a western blot of Manduca brain tissue using the BRP antibody (Fig. 1A). Before dissections, insects were kept on ice for 5 to 60 minutes. Immediately after dissection, brains were placed in Bolt TM LDS Sample Buffer (Life Technologies; Cat\#B0007) with protease inhibitor cocktail (Research Products International; Cat\# P50900) and DNase I (Invitrogen;Cat\#18068-015) and kept on ice. Tissue was homogenized using a pestle. Bolt TM Sample Reducing Agent (Life Technologies; Cat\# B0009) was added if appropriate. Samples were heated in a water bath for 10 minutes at $95^{\circ} \mathrm{C}$. We used the Novex ${ }^{\circledR}$ Bolt ${ }^{\mathrm{TM}}$ Gel Electrophoresis System (Life Technologies) with Tris-Glycine SDS Running Buffer at 165V for 2.5 hours and Bolt ${ }^{\mathrm{TM}}$ 4-12\% Bis-Tris Plus Precast Gels (BG04120BOX) to resolve proteins. We used the iBlot® Gel Transfer Device (Life Technologies; Cat\#IB1001) program P0 (20 V for 1 $\min , 23 \mathrm{~V}$ for $4 \mathrm{~min}, 25 \mathrm{~V}$ for $2 \mathrm{~min}$ ) to transfer proteins to nitrocellulose membranes (nitrocellulose iBlot ${ }^{\circledR}$ Transfer Stacks, Life Technologies; Cat\# IB3010-01). We followed the WesternBreeze ${ }^{\circledR}$ Chromogenic Western Blot Immunodetection Kit (Cat\#WB7103, anti-mouse) protocol to detect proteins. The BRP antibody was diluted 1:100. Images of membranes were taken with FluorChem Q using Alpha View Analysis Software. Western blot analysis resulted in 
a single band at the predicted height of $\sim 230 \mathrm{kDa}$ (Fig. 1A). For immunocytochemistry, sectioned tissue was incubated for 172 hours at a dilution of 1:50.

5-HT - The 5-HT antibody (Immunostar, cat\# 20079) was raised in goat against serotonin coupled to bovine serum albumin with paraformaldehyde. Pre-adsorption of the antiserum by incubating with $1 \mathrm{mg} / \mathrm{mL}$ BSA and 10:1 5-HT-BSA conjugate (Immunostar, cat\# 20081):goat anti-5-HT antibody for $24 \mathrm{hrs}$ at $4^{\circ} \mathrm{C}$ abolished all staining of Manduca AL tissue (Fig. 1B). Nonpre-adsorbed controls in which goat anti-5-HT antibody was incubated in parallel under identical conditions with the exception of the 5-HT-BSA conjugate resulted in strong immunolabeling (Fig. 1C). For immunocytochemistry, tissue was incubated at a dilution of 1:10,000 for 24 hours.

MsTH - Manduca sexta tyrosine hydroxylase (MsTH) - The MsTH antibody was raised in rabbit against MsTH, which is the rate limiting enzyme in the synthesis of DA. This antibody was generously provided by Dr. Maureen Gorman. The MsTH antibody was raised against the full length MsTH protein and immunoblot analysis reveals a single band at the predicted height (Gorman et al., 2007). AL labeling with this antibody matches immunolabeling for DA with the exception of very weak labeling to an unknown substance that is not observed within glomeruli (Dacks et al., 2012). Tissue was incubated at a dilution of 1:5,000 for 24 hours.

GABA - The GABA antibody was raised in rabbit against GABA coupled to BSA with paraformaldehyde and obtained from Sigma Aldrich (Sigma Aldrich, cat \# A2052). Controls performed by Sigma reveal that the antibody binds to GABA in a dot blot assay and not to BSA. Pre-adsorption controls were performed by incubating the rabbit anti-GABA antiserum for $24 \mathrm{hrs}$ in blocking solution (1mg/ml BSA in PBSAT) with GABA (Sigma Aldrich, cat \# A2129) at a ratio of 10:1 GABA:antiserum which abolished all staining in Manduca AL tissue (Fig. 1D). Non-pre-adsorbed controls in which rabbit anti-GABA antibody was incubated in parallel under identical conditions with the exception of GABA resulted in strong immunolabeling (Fig. 1E). For immunocytochemistry, tissue was incubated at a dilution of 1:500 for 48 hours.

Myoinhibitory peptide (MIP) - Antiserum raised in rabbit against MIP conjugated to thyroglobulin was produced by M. Eckert, Jena Germany and provided by C. Wegener, Marburg 
Germany (Predel et al., 2001). Pre-adsorption of the antiserum by incubating with $1 \mathrm{mg} / \mathrm{mL}$ BSA and 10:1 synthetic MIPVI (AWSALHGAWA): rabbit anti-MIP antibody for 24 hours at $4^{\circ} \mathrm{C}$ abolished all staining of Manduca AL tissue (Fig. 1F). Manduca sexta-MIPVI has been shown to be present in adult Manduca ALs using mass spectrometry (Utz et al., 2007). Non-pre-adsorbed controls in which rabbit anti-MIP antibody was incubated in parallel under identical conditions with the exception of the synthetic MIP $\mathrm{VI}_{\mathrm{VI}}$ resulted in strong immunolabeling (Fig. 1G). For immunocytochemistry, tissue was incubated for 48 hours at a dilution of 1:3000.

Allatotropin (ATR) - Antiserum raised in rabbit against Manduca allatotropin (Mas-AT, referred to in this paper as ATR) was kindly provided by Dr. J. Veenstra, University of Bordeaux, Talence, France; (Veenstra and Hagedorn, 1995). Specificity of Mas-AT antisera was tested previously in Manduca tissue (Veenstra and Hagedorn, 1995). Incubation of antisera for 1 hour at room temperature abolished all immunostaining in Manduca brain sections while preadsorption with FLRFamide, FMRFamide, and Dip-AST7 (all Sigma-Aldrich) for 1 hour at room temperature had no effect on immunostaining (Veenstra and Hagedorn, 1995). For immunocytochemistry, tissue was incubated for 48 hours at a dilution of 1:3000.

Allatostatin (AST) - Antiserum was raised (Reichwald et al., 1994) in rabbit against octadecapeptideallatostatin (Pratt et al., 1991), ASB2, (AYSYVSEYKALPVYNFGL-NH2) of Diploptera punctata and kindly provided by Dr. J. Veenstra, University of Bordeaux, Talence, France. Pre-adsorption controls revealed that the antisera against AKSYNFGLamide, a YXFGLamide identified from Manduca, recognizes AKSYNFGLamide, a form of allatostatin (AST) and other allatostatin-like peptides within Manduca tissue (Davis et al., 1997). For immunocytochemistry, tissue was incubated for 48 hours at a dilution of 1:3,000.

Tachykinin (TKK) - Antiserum raised in rabbit against locust tachykinin II with bovine thyroglobulin with glutaraldehyde was kindly provided to us Dr. J. Veenstra, University of Bordeaux, Talence, France. Pre-adsorption of the antiserum by incubating with $1 \mathrm{mg} / \mathrm{mL}$ BSA and 10:1 synthetic TKK1 (RAPMGFMGVR): rabbit anti-TKK antibody for $24 \mathrm{hrs}$ at $4^{\circ} \mathrm{C}$ abolished all staining of Manduca AL tissue (Fig. 1H). Non-pre-adsorbed controls in which rabbit anti-TKK antibody was incubated in parallel under identical conditions with the exception 
of the synthetic $\mathrm{TKK}_{1}$ resulted in strong immunolabeling (Fig. 1I). For immunocytochemistry, tissue was incubated for 48 hours at a dilution of 1:4,000.

Confocal Microscopy Images were scanned using an Olympus FV1000 confocal microscope with argon and green and red HeNe lasers. Fluoview software was used to adjust brightness levels, and Corel Draw 4.0 was used to organize figures.

\section{Analysis}

To quantify the relative distribution of each neuron type within a glomerulus we used two analytical approaches. The first approach was to determine the relative distance that each neuronal type projected along the proximal to distal axis of the glomerulus. The second approach was to make pair-wise comparisons of the distribution of each neuron type across the glomerulus to determine the similarity of their distribution. These approaches are depicted in Figure 2.

For each glomerulus analyzed, .tif stacks of AL scans were converted into an RBG color scheme in Fluoview software and imported into Corel Draw 4.0. Glomeruli were marked with a white dot at their most proximal, central point (Figure 2A) and cropped using the BRP staining as the boundary of glomerular area. Glomeruli were then oriented such that the white dot was at the bottom-most or " 6 o' clock" position. This was to ensure that horizontal bin 1 always corresponds to the most proximal end of the glomerulus and horizontal bin 10 to the most distal (Fig. 2B). A white bordered square with edges flush to the cropped glomerulus was applied (square shown with black border in figure 2B to visualize concept). Each cropped glomerulus and white square was exported individually as a .tif file and imported into MatLAB. Intensity values for each channel were extracted and all values that fell within the white square, but not within the glomerulus, were assigned a value of "not a number", and thus excluded from analysis. The square was then divided into a 10x10 matrix (Fig. 2C), however, given the nonsquare structure of glomeruli, not all rows or columns contained a pixel number divisible by 10 , and therefore the unequal bin sizes were randomly assigned to a bin(s). For example, one glomerulus may be 756 pixels in height by 654 pixels in width. To divide the glomerulus into 10 rows, 756 pixels are distributed into 6 rows that are 76 pixels in height and 4 rows that are 75 pixels in height. Thus, the rows are randomly assigned to having 76 vs. 75 pixels. The intensity 
values from each bin in a glomerulus were then normalized by subtracting each bin by the minimum fluorescence divided by the maximum fluorescence minus the minimum fluorescence for that glomerulus $\left(\left(X-X_{\min }\right) /\left(X_{\max }-X_{\min }\right)\right)$. Figure $2 \mathrm{D} \& E$ are intensity maps of two separate channels (MsTH-ir and 5-HT-ir for figures 2D and E respectively) from a single glomerulus run through the intensity analysis to demonstrate how each immunolabel exhibits varying levels of pixel intensities across the 10x10 grid.

For the relative distance analysis, we averaged row values in our 10x10 grid to get a resolution of 10 horizontal bins for intensity values. We used the normalized intensity values for each resulting horizontal bin (1 being most proximal, 10 most distal) for each neuron to compare the distance they extend within a glomerulus. A 1-way ANOVA for each neuron type was performed to determine at what point along the proximal to distal axis the pixel intensity values began to significantly reduce relative to the point of entry to the glomerulus. For all neuron types, with the exception of ORNs, the comparison was with relation to the proximal end of the glomerulus.

For the pair-wise correlational analysis, we calculated average normalized intensity values across our 10x10 grid of glomeruli and then plotted the intensity values as heatmaps using the heatmap function in MatLab (available athttp://www.mathworks.com/matlabcentral/?refresh=true). To quantify the similarity in glomerular distribution between labeling groups, i.e. ORN vs. PN, we ran a pair-wise linear regression analysis using Graph Pad Prism v.6.01 (Graphpad Software Incorporated) to obtain a linear relationship and $\mathrm{R}^{2}$ values for each possible combination of neuron type and immunostain. We note, the purpose of our correlation analysis was not to demonstrate whether each neuron type synapses with each other, but rather, to establish a predictive relationship determining that if ' $x$ ' appears in bin 10, how often will ' $y$ ' appear in that same bin at a similar level of intensity.

For 5-HT and MsTH, 100 sexually isomorphic glomeruli from 10 moths were used. To directly compare the intensity values for 5-HT-ir and MsTH-ir at each bin we used multiple Ttests with the Holm-Sidak method to correct for multiple comparisons in Graphpad Prism. For ORNs, PNs and GABA 10 sexually isomorphic glomeruli from 5 animals for each neuron type were used. The analyses were performed in duplicate by two different individuals on different computers and the results were consistent and reliable across person. 
All references to neuroanatomical structures use the terminology set out in Ito et al. (2014). The goal of this study is to determine the relative distribution of two extrinsic neuromodulatory neurons in relation to the primary neuron types of the AL. We do not assume connectivity based on overlap of projections, rather we are using relative distribution to provide a framework for studying the convergence of neuromodulatory inputs to this sensory network.

\section{Results:}

The AL receives serotonergic and dopaminergic input from two separate sets of extrinsic neurons. The contralaterally projecting serotonin-immunoreactive deutocerebral neuron (CSD) (Kent et al., 1987; Dacks et al., 2006a) and dopaminergic arching neurons (DAAR) (Dacks et al., 2012) project widely throughout the brain (schematized in Fig. 3A) and innervate every glomerulus of the AL. One CSD neuron cell body resides in each lateral cell cluster of the AL, projects along the medial antennal lobe tract (mALT) to the superior protocerebrum where it crosses the midline and projects anteriorly to innervate all glomeruli of the contralateral AL after having innervated both lateral horns and other protocerebral regions (Kent et al., 1987). There are two pairs of DAAR neurons, each of which projects to lateral protocerebral regions on each side of the brain as well as to both ALs where they innervate all glomeruli (Dacks et al., 2012). Thus each AL receives input from a single CSD neuron and four DAAR neurons.

\section{CSD and DAAR neurons innervate distinct yet overlapping glomerular zones}

To determine the extent of overlap between the serotonergic and dopaminergic systems within glomeruli, we labeled for 5-HT, MsTH, and BRP in adult moths. MsTH was used to immunolabel the dopaminergic DAAR neurons and the BRP antibody was used to delineate glomerular boundaries. In sexually isomorphic glomeruli, MsTH-ir extends farther distally than 5-HT which remains proximal relative to the center of the AL (Fig. 3B-D). Using multiple $t$ tests, we found that the distribution of 5-HT and DA significantly differed for the most distal bins 7-10, bin 1 being most proximal and bin 10 most distal (Fig. 3E; Multiple $t$-tests; $t=2.76$ for $\operatorname{bin} 7, \mathrm{t}=4.55$ for bin $8, \mathrm{t}=5.44$ for bin $9, \mathrm{t}=4.68$ for bin $10 ; \mathrm{df}=18 ; \mathrm{p}=0.012$ for bin $7 \mathrm{p}<0.0001$ for bin 8-10; $\mathrm{n}=100$ ). Using a 1-way ANOVA to make within modulator comparisons of intensity, we also sought to define where the distribution of each modulator began to significantly reduce in intensity along the proximal to distal axis. To determine at what point along the proximal to 
distal axis 5-HT-ir and MsTH-ir began to differ in intensity we used a 1-way ANOVA to compare intensity levels between each bin. The intensity values of the 5-HT-ir began to significantly decrease at bin 6 (Fig. 3E_, p<0.01, df=9, n=10), while the MsTH-ir intensity values do not significantly decrease until bin 9 (Fig. $3 \mathrm{E}, \mathrm{p}<0.05, \mathrm{df}=9, \mathrm{n}=10$ ). Manduca possess three sexually dimorphic glomeruli, which comprise the macroglomerular complex (MGC) in males and receive input from ORNs responsive to components of the female sex pheromone (Christensen and Hildebrand, 1987). In the MGC, MsTH also extends farther distally than 5-HT (Fig. 3F) although neither biogenic amine extended as far distally in the MGC as in the isomorphic glomeruli.

In many invertebrates, the axon terminals of ORNs innervate the distal portion of glomeruli, whereas the processes of PNs and LNs are more proximal (as reviewed in Ache and Young, 2005). To quantitatively illustrate the distribution of the three principal neuron types along the proximal to distal axis of a glomerulus in Manduca, in separate groups of moths we labeled ORNs via anterograde dye fills from the antennae, PNs via retrograde dye fills from the mushroom bodies and GABA-ir LNs and PNs via immunolabeling for GABA. Dye filled PNs and GABA-ir neurons had the greatest intensity within the most proximal 5 bins of the glomerulus, while dye filled ORNs were greatest in intensity in the most distal portion of a glomerulus (bins 7-10) (Fig. 4A). To determine at what point along the proximal to distal axis each of the 3 neuronal types began to differ in intensity, we used a 1-way ANOVA to compare intensity levels between each bin for each individual neuron type. ORN intensity values began to significantly decrease relative to the most distal bin at bin 6 (Fig. 4A, p<0.05, df=9, n=10), PN intensity values began to significantly decrease relative to the most proximal bin at bin 9 (Fig. $4 \mathrm{~A}, \mathrm{p}<0.05, \mathrm{df}=9, \mathrm{n}=10$ ) and GABA intensity values began to significantly decrease relative to the most proximal bin at bin 5 (Fig. 4A, p<0.01, df=9, n=10).

Glomeruli are irregular spheroidal structures, thus using a 10x10 grid of intensity across vertical and horizontal bins was necessary to capture the unique distribution pattern of each neuron type. In Manduca, ORNs do not innervate the glomerulus in a perfect linear progression along the distal to proximal axis, but rather form a crescent around the distal glomerular half (Fig. 4B). Conversely, PNs and GABA-ir AL neurons occupy a proximal "spheroid" within the crescent formed by the ORNs (Fig. 4C, D). To quantify the degree of similarity between these general distributions we ran a pair-wise linear regression analysis of the labeling intensity for 
each neuronal type within a glomerulus. The innervation patterns of ORNs and PNs showed a significant negative relationship (Fig. 4E; $\mathrm{r}^{2}=0.3251, \mathrm{p}<0.0001$ ) as did the patterns for ORNs and GABA (Fig. 4F; $\mathrm{r}^{2}=0.6494, \mathrm{p}<0.0001$ ). Finally, the innervation patterns of PNs and GABA-ir AL neurons showed a significant positive relationship (Fig. 4G; $r^{2}=0.2444$, $\mathrm{p}<0.0001)$.

We therefore sought to determine if the differences in innervation pattern of the CSD and DAAR neurons were reflective of differences in the glomerular regions occupied by the ORNs, LNs and PNs. Antennal nerve fills were used to anterogradely fill the axons of ORNs to the AL (Fig. 5A). Subsequent immunocytochemical labeling for 5-HT and MsTH revealed that, consistent with Sun et al. (1993), the 5-HT-ir processes remained proximal and had little overlap with the ORNs (Fig. 3B), while ORNs almost completely overlapped with MsTH-ir (Fig. 3C). An almost complete lack of overlap between 5-HT-ir and ORNs was also observed in the MGC (Fig. 3D-F). Using a 10x10 grid of intensity across vertical and horizontal bins to determine the average distribution of ORNs, 5-HT-ir and MsTH-ir, we found that ORNs occupy the most distal bins as shown in (Fig. 5G), while 5-HT-ir and MsTH-ir both innervate the most proximal bins (Fig. 5H-I) with MsTH-ir extending farther distally into the glomerulus (Fig. 5I). To quantify the degree of similarity between ORNs and 5-HT-ir we ran a pair-wise linear regression analysis of the labeling intensity for each neuronal type within a glomerulus. Both 5-HT-ir and MsTH-ir showed a negative relationship with ORNs (Fig. 5J-K; $\mathrm{r}^{2}=0.6162, \mathrm{p}<0.0001$ and $\mathrm{r}^{2}=0.5133$, $\mathrm{p}<0.0001$ respectively). To illustrate the distribution of the CSD and DAAR processes relative to PN dendrites, mushroom body dye fills were used to retrogradely fill PN processes in the AL (Fig. 6A). The CSD and DAAR processes intermingle with PN dendrites, but only the DAAR neurons extend more distally beyond the boundary of the PNs (Figure 4B-G). Both 5-HT-ir and MsTH-ir showed a positive relationship with PNs (Fig. 6E-F; $\mathrm{r}^{2}=0.2810, \mathrm{p}<0.0001, \mathrm{r}^{2}=$ 0.09240, $\mathrm{p}=0.0022$ respectively).

Distribution of the CSD and DAAR neurons relative to the GABAergic and peptidergic AL neurons

To illustrate the relative distribution of the CSD processes with the diverse modulatory environment of the AL, we compared the distribution of 5-HT-ir to the intrinsic modulatory network of GABAergic and peptidergic AL neurons in the lateral cell cluster. Because the most 
reliable GABA antibody and all of the available neuropeptide antibodies were raised in rabbit, we did not examine their relative distribution with MsTH-ir as this antibody was also raised in rabbit. There are 460 GABAergic cell bodies in the lateral cell cluster, of which 360 are LNs and 100 are PNs (Hoskins et al., 1986), so GABA-ir provides a method to highlight a major proportion of cells in the lateral cell cluster of Manduca. Consistent with (Hoskins et al., 1986), GABA-ir processes were denser at the proximal region of isomorphic glomeruli and sparser distally (Fig. 4A\&D). Triple immunolabeling (Fig. 7A) revealed extensive overlap of 5-HT-ir within the denser GABA-ir in the proximal region of the isomorphic glomeruli (Fig. 7B). As in Figure 3, the BRP antibody was used to delineate glomerular boundaries. However, the CSD neurons did not extend their processes distally past the boundary at which the GABA-ir processes become spare (Fig. 7C, D). GABA showed a significant positive relationship with 5HT (Fig. 7E; $\mathrm{r}^{2}=0.4436, \mathrm{p}<0.0001$ ).

Despite the large number of GABAergic LNs, not all LNs in the ALs of Manduca are GABAergic (Reisenman et al., 2011) and many LNs (and some PNs) express a variety of different neuropeptides (Homberg et al., 1990; Utz and Schachtner, 2005; Reisenman et al., 2011). Thus, antibodies against several neuropeptides were used to highlight the processes of a smaller number of LNs and PNs than are labeled with GABA-ir. Figure 8 depicts the arborizations of the CSD neuron and 4 populations of peptidergic neurons: tachykinin-ir (TKK; Fig. 8A-F), allatotropin-ir (ATR; Fig. 8G-I), allatostatin-ir (AST; Fig. 8J-L) and myoinhibitory peptide-ir (MIP; Fig. 8M-P). Within the isomorphic glomeruli (Fig. 8; central column) all four populations of peptidergic neurons overlap with 5-HT-ir but all extend farther distally. To the best of our knowledge, this is the first description of TKK-ir in the AL of Manduca. Each AL possesses $\sim 9$ TKK-ir cell bodies which together innervate all glomeruli (Fig. 8A-B). While some TKK-ir cell bodies appear to be LNs according to their morphology, there is a process leaving the AL (Fig. 8C), demonstrating that some TKK-ir cells may be PNs or even centrifugal neurons. A small number of ATR-ir neurons in the lateral cell cluster are also PNs and a few centrifugal AST-ir neurons innervate the AL and arborize in glomeruli (Utz and Schachtner, 2005; Utz et al., 2007; Utz et al., 2008). Similar to the GABA-ir, ATR-ir was denser at the proximal core of isomorphic glomeruli as well as the MGC, but did extend distally with sparer processes (Fig 8I). The AST-ir was also denser proximally, although not nearly to the same extent as the GABA-ir or the ATR-ir (Fig. 8K). MIP-ir and TKK-ir were generally uniform in density. Utz et al., 2007 
reported that the majority of MIP-ir neurons are LNs based on their morphology, and our results were consistent with this finding in that the MIP-ir cell bodies were relatively large, on average 20 um compared to PN cell bodies in the lateral cell cluster (6.2-8.9um; Homberg et al., 1988). MIP-ir was uniformly distributed throughout isomorphic glomeruli, extending farther distally than 5-HT in both the isomorphic glomeruli and the MGC (Fig. 8N-O).

\section{Discussion:}

Neuromodulation provides a network with the capacity to produce a wide range of output under different conditions. Without this flexibility the same network would need exponentially more connections to achieve the same endpoint. A variety of external and internal conditions trigger the release of neuromodulators and as a consequence each network must dynamically integrate the influences of many neuromodulators. In this study, we described the relative glomerular distribution of two extrinsic modulatory systems within the AL of Manduca, their overlap with input neurons, output neurons and intrinsic sources of neuromodulators. The differences in the glomerular arborizations of the CSD and DAAR neurons (releasing 5-HT and DA respectively) suggest that each modulator targets partially overlapping functional groups of neurons. While both the processes of the CSD and DAAR neurons overlap extensively with LNs and PNs, the DAAR neurons extended sufficiently distally to overlap with the axon terminals of ORNs while the CSD neurons remained proximal and had very little overlap with ORNs. Based on their anatomical distributions within glomeruli, the CSD neurons are less likely to have significant presynaptic influence on ORNs while the DAAR neurons have the potential to influence all three major intrinsic neuron types in the AL.

We employed two approaches to analyze the distribution of 5-HT-ir and MsTH-ir with each other and with the three major AL neuron types. To determine if CSD and DAAR neurons differ in the distance that they extend within a glomerulus, we divided glomeruli into 10 bins along the proximal to distal axis and made comparisons of immunolabeling intensity at each bin. This allowed us to determine that these two modulatory populations had similar levels of innervation of the proximal glomerular regions, but differed in their distribution within the distal region. Specifically, the intensity values for 5-HT-ir began to significantly decrease within the distal $40 \%$ of the glomerulus, while MsTH-ir did not significantly decrease until the distal $20 \%$

of the glomerulus (Fig. 3E). Although their decrease was much more gradual than that of the 5- 
HT-ir, PNs and GABA-ir neurons also decreased in their innervation in the distal glomerular region (Fig. 4A). ORNs, on the other hand had their greatest intensity within the distal $40 \%$ of the glomeruli and decreased proximally. Pair-wise comparisons of each neuronal type revealed that overall, ORNs typically showed a negative relationship with the other neuronal types (Figs. 4E,F and 5J,K), whereas all other pair-wise comparisons showed a positive relationship. Despite the negative relationship of overall distribution of MsTH-ir with ORNs across the entire glomerulus, MsTH-ir neurons had a higher intensity than 5-HT-ir, PNs and GABA-ir neurons within the distal most regions of the glomerulus occupied by the ORNs (Fig. 3E and 4A).

While overlapping projections converging in the same glomerular sub-region suggest potential interaction of neuromodulators, anatomy is only the first step in determining a basis for the convergent physiological effects of neuromodulation. The physiological convergence of neuromodulators depends upon receptor expression of the pre or postsynaptic neuron. Consistent with our finding that the distributions of 5-HT-ir and GABA-ir overlapped, GABAergic LNs within the AL of Manduca express the Ms5HT1A receptor (Dacks et al., 2013) and presumed LNs grown in cell culture, and therefore isolated from any synaptic input, are directly modulated by 5-HT (Mercer et al 1995). Ms5HT1B, Ms5HT2 and Ms5HT7 receptor mRNAs are expressed in the AL (Dacks et al., 2006b; Dacks et al., 2013) as are the mRNAs for the MsDop1, MsDop2 and MsINDR receptors (Dacks et al., 2012). However, receptor expression by specific neuronal types of the AL (i.e. LN vs. PN) of Manduca is still relatively unexplored. Receptors for 5-HT and DA have the capacity to converge upon the same biochemical cascades as they all couple to adenylyl cyclase to affect cAMP levels (Saudou et al., 1992; Colas et al., 1995; Mustard et al., 2005). However, the biochemical consequences of activating 5-HT and DA receptors also have the opportunity to diverge, as the 5-HT1 and 5-HT2 receptor subtypes affect $\mathrm{IP}_{3}$ pathways while DA receptors and the 5-HT7 receptor do not (Saudou et al., 1992; Mustard et al., 2005).

The potential for convergent modulatory influence is present in all insect ALs. Most, if not all glomeruli receive input from 5-HT (Kent et al., 1987; Salecker and Distler, 1990; Sun et al., 1993; Wegerhoff, 1999; Hill et al., 2002; Dacks et al., 2006a; Roy et al., 2007) and neuropeptides (Carlsson et al., 2010; Kromann et al., 2013; Siju et al., 2013) suggesting that neuromodulation is ubiquitous within the AL. In the AL of Manduca, the CSD and DAAR neurons both ramify in all glomeruli (Kent et al., 1987; Dacks et al., 2006a; Dacks et al., 2012) however, we demonstrate their relative projection patterns reveal the potential for convergent 
modulation on LNs and PNs and differences in their ability to presynaptically modulate ORNs. This is consistent with work in Drosophila showing that presynaptic inhibition of ORNs by GABAergic LNs is enhanced by 5-HT despite 5-HT having no direct effect on ORNs (Dacks et al., 2009). This suggests that 5-HT acts, in part, through lateral interactions exerted by LNs to shape odor evoked responses. Furthermore, the glomeruli to which the CSD neurons project in the AL of Drosophila are tightly regulated by ephrin signaling pathways and the disruption of this developmental control has significant consequences for odor-guided behavior (Singh et al., 2013). This suggests that the stereotyped distribution of CSD processes has functional consequences for odor-processing. In Manduca, 5-HT has odor specific effects on AL neuron responses, further suggesting that 5-HT modulates the capacity of LNs to refine the transfer of information between ORNs and PNs (Dacks et al., 2008). However, this does not suggest that ORNs are not affected by 5-HT from sources other than the CSD neurons. ORNs are continuously subjected to the influence of circulating neuromodulators in the hemolymph (Schendzielorz et al., 2015) and the mRNA for four of the Manduca 5-HT receptors are expressed in the antennae, far from the axons of CSD neurons in the AL (Dacks et al., 2006b; Dacks et al., 2013), although it is possible that 5-HT release from the CSDs may act at a distance to influence ORNs in an as yet unobserved manner.

Although 5-HT and DA are likely released under different behavioral contexts, they both generally enhance odor evoked responses of AL neurons (Kloppenburg and Hildebrand, 1995; Dacks et al., 2008; Kloppenburg and Mercer, 2008; Dacks et al., 2012), although their effects differ in several ways. The levels of 5-HT in the ALs of Manduca fluctuate throughout the day peaking at dawn and dusk (Kloppenburg et al., 1999), when Manduca perform the majority of their odor-guided behaviors (Lingren et al., 1977). Furthermore, injection of 5-HT into cabbage looper moths broadens the range of the day in which male moths will fly to female pheromone (Linn and Roelofs, 1986). This suggests that the release of 5-HT by the CSD neurons is at least, in part, regulated by the waking state. Physiologically, bath application of 5-HT reduces two $\mathrm{K}^{+}$ conductances, a sustained $\mathrm{I}_{\mathrm{KV}}$-like conductance and a more transient $\mathrm{I}_{\mathrm{A}}$-like conductance, and lowers the voltage of inactivation for the $\mathrm{I}_{\mathrm{A}}$-like conductance in PNs (Kloppenburg et al., 1999) and cultured LNs (Mercer et al., 1995). As a consequence, the resistance (and therefore the excitability) of LNs and PNs is increased by 5-HT (Kloppenburg and Hildebrand, 1995) and the odor-evoked responses of AL neurons is also increased (Kloppenburg et al., 1999; Dacks et al., 
2008). Another consequence of the effects 5-HT is an increase in the slope of odor-evoked responses as a function of odor concentration (Dacks et al., 2008) suggesting that 5-HT modulates the gain of the AL.

The context for the release of DA within the AL is associated with the context of aversive stimuli, as injection of DA receptor antagonists into the ALs blocks aversive conditioning (Dacks et al., 2012), but not appetitive conditioning (Riffell et al., 2013). Similar to 5-HT, bath application of DA increased the maximal odor-evoked firing rate of extracellularly recorded AL neurons, but did not affect response gain (Dacks et al., 2012) as was observed for 5-HT (Dacks et al., 2008). DA and 5-HT also differ in their effects on the temporal dynamics of odor evoked responses. While 5-HT generally increased odor evoked response duration (Kloppenburg et al., 1999; Dacks et al., 2008), DA reduced the post-excitation period of spike suppression in PNs called the $I_{2}$ phase (Dacks et al., 2012) that typically lasts between 10ms and 1.5 seconds (Christensen et al., 1996). The $\mathrm{I}_{2}$ phase affects the fidelity with which PNs can encode the temporal dynamics of odor stimuli and blocking the $\mathrm{I}_{2}$ phase altogether negatively impacts the ability of moths to track odor plumes (Lei et al., 2009), suggesting that DA may modulate the coding of stimulus timing within the AL. Although the physiological effects of both modulators on a single AL neuron have not been examined, 50\% of AL neurons are affected by 5-HT (Kloppenburg and Hildebrand, 1995; Mercer et al., 1995; Dacks et al., 2008) and 60\% of AL neurons are affected by DA (Dacks et al., 2012), suggesting that at least $10 \%$ of AL neurons are affected by both modulators. The AL must therefore integrate the effects of multiple neuromodulators, which each have distinct effects information processing and sensory coding.

Neuromodulatory neurons can be extrinsic (projecting from outside the network) or intrinsic (part of the network) to a system (Katz, 1999). In the AL, LNs intrinsic to the network refine the transfer of information from ORNs to PNs via a diverse set of pre- and post-synaptic mechanisms. In Manduca, GABAergic LNs mediate a variety of tasks including maintaining temporal fidelity, contrast enhancement between glomeruli and intraglomerular and interglomerular synchrony (Waldrop et al., 1987; Christensen et al., 1998; Lei et al., 2002; Lei et al., 2009; Tripathy et al., 2010; Daly et al., 2011; Martin et al., 2011). Furthermore, LNs coexpress a diverse array of neuropeptides for which a few have been demonstrated to modulate olfactory processing (Ignell et al., 2009; Root et al., 2011). In Drosophila, LNs co-transmit GABA and tachykinin (Carlsson et al., 2010), both of which mediate presynaptic gain control of 
ORNs via GABAb receptors (Olsen and Wilson, 2008; Root et al., 2008) and tachykinin receptors (Ignell et al., 2009). Unlike classical small neurotransmitters such as GABA, neuropeptides work on at least a 100-fold longer time scale (Salio et al., 2006). Thus, the corelease of both GABA and neuropeptides in Manduca may provide layers of inhibition exerted over different time courses. While LNs represent a source of neuromodulation exerted within the context of recent network activation, the CSD and DAAR neurons represent an extrinsic source of neuromodulation exerted based on the general physiological state of the individual moth. All of these neuromodulatory neurons had a high degree of overlap (Fig. 3, 8) within the glomeruli suggesting that olfactory processing is dynamically shaped by multiple modulatory inputs, both extrinsic and intrinsic.

Sensory systems adjust how they encode information about the environment under a variety of different circumstances. Different internal and external conditions exert constraints on the physiological needs of the individual animal and the capacity to modify sensory processing in an appropriate manner is, in part, carried out by the integration of multiple sources of modulation. The integration of multiple modulators within a single network, neuron or second messenger pathway allows dynamic control over biophysical properties and vesicle release on both short and long time scales potentially with non-linear effects. In this manner, integrated modulation increases cellular dynamics, expanding the processing power of a network. Our data reveals that 5-HT and DA may converge upon the same neuron types, providing the potential for their integrated effects. Intrinsic and extrinsic modulatory neurons represent a significant proportion of the AL network which speaks to the importance of neuromodulation for information processing within the AL. Overall, the integration of modulators within the

olfactory system may serve as a layered source of modulation for dynamically adjusting sensory processing in a complex, odor filled environment.

\section{Acknowledgements:}

We thank Jing Wang for helping with data collection support, as well as the other members of the Dacks lab for their support. The TKK, AST, and ATR antibodies were provided by Dr. Jan Veenstra, the MIP antibody was provided by Dr. Christian Wegener and developed by Dr. Manfred Eckert, and the MsTH antibody was provided by Dr. Maureen Gorman. We thank Dr. Kevin Daly, Dr. Sarah Farris, and Tyler Sizemore for providing critical feedback on earlier versions of this manuscript. 


\section{Works cited:}

Ache BW, Young JM. 2005. Olfaction: diverse species, conserved principles. Neuron 48:417-30.

Bargmann CI. 2012. Beyond the connectome: how neuromodulators shape neural circuits. Bioessays $34: 458-65$.

Bell RA, \& Joachim FG. 1976. Techniques for rearing laboratory colonies of tobacco hornworms and pink bollworms. Annals of the Entomological Society of America, 69(2), 365-373.

Brezina V. 2010. Beyond the wiring diagram: signalling through complex neuromodulator networks. Philos Trans R Soc Lond B Biol Sci 365:2363-74.

Carlsson MA, Diesner M, Schachtner J, Nässel DR. 2010. Multiple neuropeptides in the Drosophila antennal lobe suggest complex modulatory circuits. J Comp Neurol 518:3359-80.

Champagne MB, Edwards KA, Erickson HP, \& Kiehart DP. 2000. Drosophila stretchin-MLCK is a novel member of the Titin/Myosin light chain kinase family. J Mol Biol, 300(4), 759-777.

Christensen TA, \& Hildebrand JG. 1987. Male-specific, sex pheromone-selective projection neurons in the antennal lobes of the moth Manduca sexta. J Comp Physiol A, 160(5), 553-569.

Christensen TA, Waldrop BR, \& Hildebrand JG. 1998. Multitasking in the olfactory system: contextdependent responses to odors reveal dual GABA-regulated coding mechanisms in single olfactory projection neurons. J neurosci, 18(15), 5999-6008.

Colas JF, Launay JM, Kellermann O, Rosay P, \& Maroteaux L. 1995. Drosophila 5-HT2 serotonin receptor: coexpression with fushi-tarazu during segmentation. PNAS, 92(12), 5441-5445.

Dacks AM, Christensen TA, Hildebrand JG. 2008. Modulation of olfactory information processing in the antennal lobe of Manduca sexta by serotonin. J Neurophysiol 99:2077-85.

Dacks AM, Green D, Root C, Nighorn A, Wang J. 2009. Serotonin Modulates Olfactory Processing in the Antennal Lobe of Drosophila. J Neurogenet 1-13.

Dacks AM, Christensen TA, Hildebrand JG. 2006. Phylogeny of a Serotonin- Immunoreactive Neuron in the Primary Olfactory Center of the Insect Brain. J Comp Neurol746:727-746.

Dacks AM, Dacks JB, Christensen TA, \& Nighorn AJ. 2006. The cloning of one putative octopamine receptor and two putative serotonin receptors from the tobacco hawkmoth, Manduca sexta. Insect biochemistry and molecular biology, 36(9), 741-747.

Dacks AM, Reale V, Pi Y, Zhang W, Dacks JB, Nighorn AJ, Evans PD. 2013. A characterization of the Manduca sexta serotonin receptors in the context of olfactory neuromodulation. PLoS One 8:e69422.

Dacks AM, Reisenman CE, Paulk AC, Nighorn AJ. 2010. Histamine-immunoreactive local neurons in the antennal lobes of the hymenoptera. J Comp Neurol 518:2917-33. 
Dacks AM, Riffell JA, Martin JP, Gage SL, Nighorn AJ. 2012. Olfactory modulation by dopamine in the context of aversive learning. J Neurophysiol 108:539-50.

Daly KC, Galán RF, Peters OJ, \& Staudacher EM. 2011. Detailed characterization of local field potential oscillations and their relationship to spike timing in the antennal lobe of the moth Manduca sexta. Front neuroeng, 4.

Daly KC, Kalwar F., Hatfield M., Staudacher E, \& Bradley SP. 2013. Odor detection in Manduca sexta is optimized when odor stimuli are pulsed at a frequency matching the wing beat during flight. PloS one, 8(11), e81863.

Davis NT, Veenstra JA, Feyereisen R. and Hildebrand JG. 1997. Allatostatinlike immunoreactive neurons of the tobacco hormworm, Manduca sexta, and isolation and identification of a new neuropeptide related to cockroach allatostatins. J Comp Neurol 385:265-284.

Fontanini A, Katz, DB. 2006. State-dependent modulation of time-varying gustatory responses. J Neurophysiol 96:3183-93.

Getting PA. 1989. Emerging principles governing the operation of neural networks. Ann rev Neurosci, 12(1), 185-204.

Gorman MJ, An C, Kanost MR. 2007. Characterization of tyrosine hydroxylase from Manduca sexta. Insect Biochem Mol Biol 37:1327-37.

Homberg U, Montague RA, \& Hildebrand JG. 1988. Anatomy of antenno-cerebral pathways in the brain of the sphinx moth Manduca sexta. Cell and tissue research, 254(2), 255-281.

Hill ES, Iwano M, Gatellier L, \& Kanzaki R 2002. Morphology and physiology of the serotoninimmunoreactive putative antennal lobe feedback neuron in the male silkmoth Bombyx mori. Chem senses, 27(5), 475-483.

Hofbauer A. 1991. Eine Bibliothek monoklonaler Antikörper gegen das Gehirn von Drosophila melanogaster. Habilitation thesis. University of Würzburg, Würzburg, Germany.

Homberg U, Kingan TG, \& Hildebrand JG. 1990. Distribution of FMRFamide-like immunoreactivity in the brain and suboesophageal ganglion of the sphinx moth Manduca sexta and colocalization with SCPB-, BPP-, and GABA-like immunoreactivity. Cell and tissue research, 259(3), 401-419.

Homberg U, Montague RA, \& Hildebrand JG. 1988. Anatomy of antenno-cerebral pathways in the brain of the sphinx moth Manduca sexta. Cell and tissue research, 254(2), 255-281.

Hoskins SG, Homberg U, Kingan TG, Christensen TA, \& Hildebrand JG. 1986. Immunocytochemistry of GABA in the antennal lobes of the sphinx moth Manduca sexta. Cell and tissue research, 244(2), 243-252.

Huetteroth W, \& Schachtner J. 2005. Standard three-dimensional glomeruli of the Manduca sexta antennal lobe: a tool to study both developmental and adult neuronal plasticity. Cell and tissue research, 319(3), 513-524. 
Hurley LM, Hall IC. 2011. Context-dependent modulation of auditory processing by serotonin. Hear Res 279:74-84.

Ignell R, Root CM, Birse RT, Wang JW, Nässel, D. R., \& Winther, Å. M. 2009. Presynaptic peptidergic modulation of olfactory receptor neurons in Drosophila. PNAS, 106(31), 13070-13075.

Ito, K., Shinomiya, K., Ito, M., Armstrong, J. D., Boyan, G., Hartenstein, V., ... \& Insect Brain Name Working Group. 2014. A systematic nomenclature for the insect brain. Neuron, 81(4), 755-765.

Kaczmarek LK, \& Levitan IB. (Eds.). 1987. Neuromodulation: the biochemical control of neuronal excitability. Oxford University Press.

Katz PS, and Edwards DH. 1999. Beyond neurotransmission. New York: Oxford University Press,

Kent KS, Hoskins SG, Hildebrand JG. 1987. A novel serotonin-immunoreactive neuron in the antennal lobe of the sphinx moth Manduca sexta persists throughout postembryonic life. J Neurobiol 18:45165.

Kloppenburg P, Ferns D, Mercer AR. 1999. Serotonin enhances central olfactory neuron responses to female sex pheromone in the male sphinx moth Manduca sexta. J Neurosci 19:8172-81

Kloppenburg P, \& Heinbockel T. 2000. 5-Hydroxy-tryptamine modulates pheromone-evoked local field potentials in the macroglomerular complex of the sphinx moth Manduca sexta. J Exp Biol, 203(11), 1701-1709

Kloppenburg P, Hildebrand JG. 1995. Neuromodulation by 5-hydroxytryptamine in the antennal lobe of the sphinx moth Manduca sexta. J Exp Biol 198:603-11.

Kloppenburg P, Mercer AR. 2008. Serotonin modulation of moth central olfactory neurons. Annu Rev Entomol 53:179-90.

Komuniecki R, Hapiak V, Harris G, Bamber B. 2014. Context-dependent modulation reconfigures interactive sensory-mediated microcircuits in Caenorhabditis elegans. Curr Opin Neurobiol 29C:17-24.

Kromann SH, Hansson BS, Ignell R. 2013. Distribution of neuropeptides in the antennal lobes of male Spodoptera littoralis. Cell Tissue Res 354:431-40.

Kupfermann I. 1979. Modulatory actions of neurotransmitters. Annu Rev Neurosci 2:447-65.

Lei H, Christensen T a, Hildebrand JG. 2002. Local inhibition modulates odor-evoked synchronization of glomerulus-specific output neurons. Nat Neurosci 5:557-65.

Lei H, Riffell J a, Gage SL, Hildebrand JG. 2009. Contrast enhancement of stimulus intermittency in a primary olfactory network and its behavioral significance. J Biol 8:21.

Lingren PD, Greene GL, Davis DR, Baumhover AH, Henneberry TJ. 1977. Nocturnal behavior of four lepidopteran pests that attack tobacco and other crops. Annals of the Entomological Society of America, 70(2), 161-167. 
Linn CE, Roelofs WL. 1986. Modulatory effects of octopamine and serotonin on male sensitivity and periodicity of response to sex pheromone in the cabbage looper moth, Trichoplusia ni. Archives of insect biochemistry and physiology, 3(2), 161-171.

Marder E. 2012. Neuromodulation of neuronal circuits: back to the future. Neuron 76:1-11.

Martin JP, Lei H, Riffell JA, Hildebrand JG. 2013. Synchronous firing of antennal-lobe projection neurons encodes the behaviorally effective ratio of sex-pheromone components in male Manduca sexta. J Comp Physiol A Neuroethol Sens Neural Behav Physiol 199:963-79.

Mercer AR, Hayashi JH, Hildebrand JG. 1995. Modulatory effects of 5-hydroxytryptamine on voltageactivated currents in cultured antennal lobe neurones of the sphinx moth Manduca sexta. Journal exp biol, 198(3), 613-627.

Mercer AR, Kirchhof BS, Hildebrand JG. 1996. Enhancement by serotonin of the growth in vitro of antennal lobe neurons of the sphinx moth Manduca sexta. J Neurobiol 29:49-64.

Mustard JA, Beggs KT, Mercer AR. 2005. Molecular biology of the invertebrate dopamine receptors. Arch Insect Biochem Physiol 59:103-17.

Nusbaum MP, Blitz DM. 2012. Neuropeptide modulation of microcircuits. Curr Opin Neurobiol 22:592601.

Nusbaum MP., Marder, E. 1989. A Modulatory Proctolin-Containing Modulation of Rhythmic Motor Activity. J Neurosci:1600-1607.

Olsen SR, Wilson RI. 2008. Lateral presynaptic inhibition mediates gain control in an olfactory circuit. Nature 452:956-60.

Pratt GE, Farnsworth DE, Fok KF, Siegel NR, McCormack AL, Shabanowitz J, Hunt DF, Feyereisen R. 1991. Identity of a second type of allatostatin from cockroach brains: an octadecapeptide amide with a tyrosine-rich address sequence. PNAS S A 88:2412-6.

Predel R, Rapus J, Eckert M. 2001. Myoinhibitory neuropeptides in the American cockroach. Peptides 22:199-208.

Reisenman CE, Dacks AM, Hildebrand JG. 2011. Local interneuron diversity in the primary olfactory center of the moth Manduca sexta. J Comp Physiol A Neuroethol Sens Neural Behav Physiol 197:653-65.

Reichwald K, Unnithan GC, Davis NT, \& Feyereisen R. 1994. Expression of the allatostatin gene in endocrine cells of the cockroach midgut. PNAS, 91(25), 11894-11898.

Riffell JA, Lei H, Abrell L, Hildebrand JG. 2013. Neural basis of a pollinator's buffet: olfactory specialization and learning in Manduca sexta. Science, 339(6116), 200-204.

Root CM, Masuyama K, Green DS, Enell LE, Nässel DR, Lee C-H, Wang JW. 2008. A presynaptic gain control mechanism fine-tunes olfactory behavior. Neuron 59:311-21. 
Root CM, Ko KI, Jafari A, \& Wang JW. (2011). Presynaptic facilitation by neuropeptide signaling mediates odor-driven food search. Cell, 145(1), 133-144.

Rospars JP, \& Hildebrand JG 2000. Sexually dimorphic and isomorphic glomeruli in the antennal lobes of the sphinx moth Manduca sexta. Chem senses, 25(2), 119-129.

Roy B, Singh AP, Shetty C, Chaudhary V, North A, Landgraf M, Vijayraghavan K, Rodrigues V. 2007. Metamorphosis of an identified serotonergic neuron in the Drosophila olfactory system. Neural Dev 2:20.

Salecker I, \& Distler P. 1990. Serotonin-immunoreactive neurons in the antennal lobes of the American cockroach Periplaneta americana: light-and electron-microscopic observations. Histochemistry, 94(5), 463-473.

Salio, C, Lossi L, Ferrini F, \& Merighi A. 2006. Neuropeptides as synaptic transmitters. Cell and tissue research, 326(2), 583-598.

Saudou F, Boschert U, Amlaiky N, Plassat J L, \& Hen R. 1992. A family of Drosophila serotonin receptors with distinct intracellular signalling properties and expression patterns. The EMBO journal, 11(1), 7.

Schendzielorz T, Schirmer K, Stolte P, \& Stengl M. 2015. Octopamine regulates antennal sensory neurons via daytime-dependent changes in cAMP and IP3 levels in the hawkmoth Manduca sexta. PloS one, 10(3), e0121230.

Siju KP, Reifenrath A, Scheiblich H, Neupert S, Predel R, Hansson BS, Schachtner J, Ignell R. 2014. Neuropeptides in the antennal lobe of the yellow fever mosquito, Aedes aegypti. J Comp Neurol 522:592-608.

Singh AP, Das RN, Rao G, Aggarwal A, Diegelmann S, Evers JF, ... Vijayraghavan K. 2013. Sensory neuron-derived eph regulates glomerular arbors and modulatory function of a central serotonergic neuron. PLoS Genet, 9(4), e1003452.

Su C-Y, Wang JW. 2014. Modulation of neural circuits: how stimulus context shapes innate behavior in Drosophila. Curr Opin Neurobiol 29C:9-16.

Sun XJ, Tolbert LP, Hildebrand JG. 1993. Ramification pattern and ultrastructural characteristics of the serotonin-immunoreactive neuron in the antennal lobe of the moth Manduca sexta: a laser scanning confocal and electron microscopic study. J Comp Neurol 338:5-16.

Tripathy SJ, Peters OJ, Staudacher EM, Kalwar FR., Hatfield MN, \& Daly KC. 2010. Odors pulsed at wing beat frequencies are tracked by primary olfactory networks and enhance odor detection. Front cell neurosci, 4.

Utz S, Huetteroth W, Wegener C, Kahnt J, Predel R, Schachtner J 2007. Direct peptide profiling of lateral cell groups of the antennal lobes of Manduca sexta reveals specific composition and changes in neuropeptide expression during development. Devel neurobiol, 67(6), 764-777. 
Utz S, Huetteroth W, Vömel M, Schachtner J 2008. Mas-allatotropin in the developing antennal lobe of the sphinx moth Manduca sexta: distribution, time course, developmental regulation, and colocalization with other neuropeptides. Devel neurobiol, 68:123-142

Utz S, \& Schachtner J. 2005. Development of A-type allatostatin immunoreactivity in antennal lobe neurons of the sphinx moth Manduca sexta. Cell and tissue research, 320(1), 149-162.

Veenstra JA, \& Hagedorn HH. 1993. Sensitive enzyme immunoassay for Manduca allatotropin and the existence of an allatotropin-immunoreactive peptide in Periplaneta americana. Archives of insect biochemistry and physiology, 23(3), 99-109.

Wagh DA, Rasse TM, Asan E, Hofbauer A, Schwenkert I, Dürrbeck H, Buchner S, Dabauvalle M-C, Schmidt M, Qin G, Wichmann C, Kittel R, Sigrist SJ, Buchner E. 2006. Bruchpilot, a protein with homology to ELKS/CAST, is required for structural integrity and function of synaptic active zones in Drosophila. Neuron 49:833-44.

Waldrop B, Christensen TA, \& Hildebrand JG. 1987. GABA-mediated synaptic inhibition of projection neurons in the antennal lobes of the sphinx moth, Manduca sexta. Journal of Comparative Physiology A, 161(1), 23-32.

Wegerhoff R. 1999. GABA and serotonin immunoreactivity during postembryonic brain development in the beetle Tenebrio molitor. Microscopy research and technique, 45(3), 154-164. 


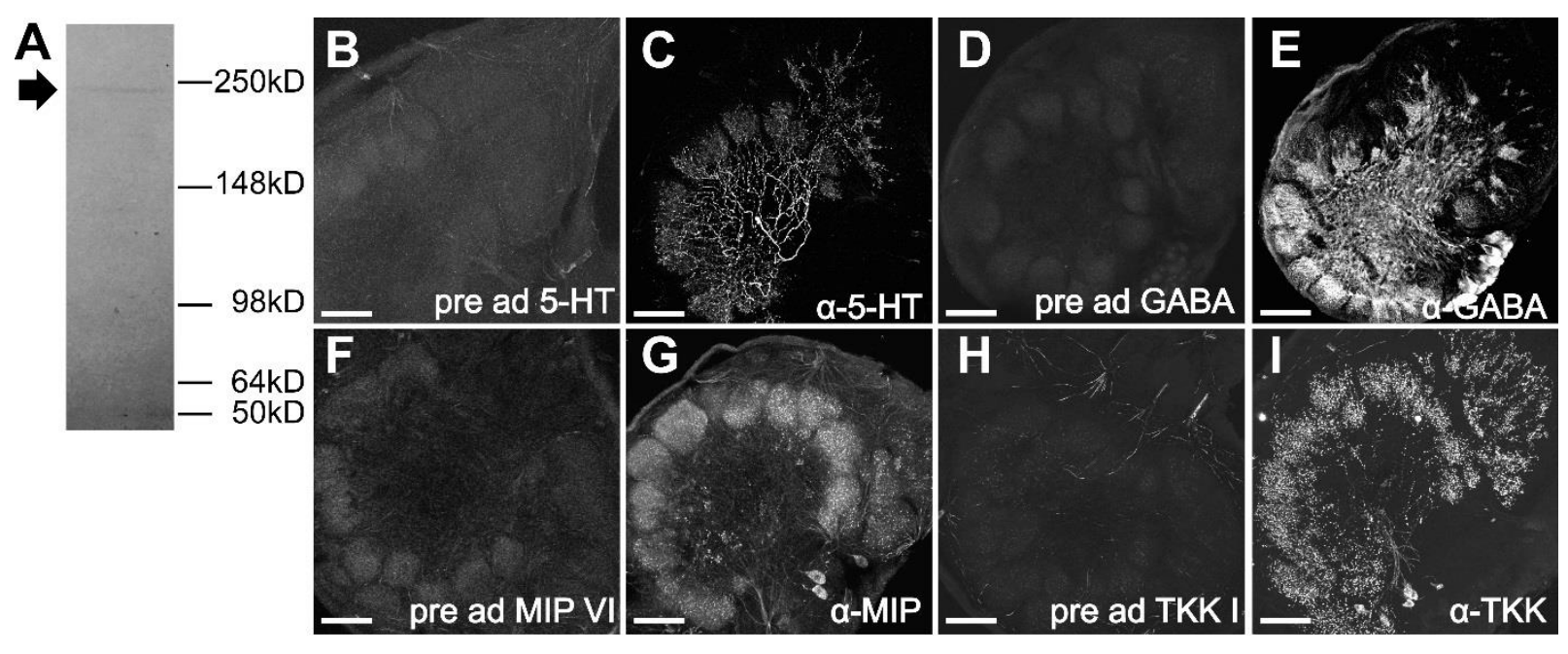

Figure 1. Western blot of BRP antibody using Manduca brain tissue and pre-adsorption controls for the 5-HT, GABA, MIP and TKK antibodies. A:

Western blots of Manduca brain tissue with the BRP antibody resulted in a single band at the predicted height for the Manduca homologue of the ELKS/CAST protein protein $(\sim 234 \mathrm{kDa})$. The BRP antibody was used to mark glomerular outlines. B: Pre-adsorption of the goat anti-5-HT antiserum by incubating with $1 \mathrm{mg} / \mathrm{mL}$ BSA and 10:1 5-HT-BSA conjugate:goat anti-5-HT antibody for $24 \mathrm{hrs}$ at $40 \mathrm{C}$ abolished all staining of Manduca AL tissue. C: Non-pre-adsorbed controls in which goat anti-5-HT antibody was incubated in parallel under identical conditions with the exception of the 5-HT-BSA conjugate resulted in strong immunolabeling. D: Pre-adsorption of the rabbit anti-GABA antiserum by incubating with $1 \mathrm{mg} / \mathrm{mL}$ BSA and 10:1 GABA:rabbit anti-GABA antibody for $24 \mathrm{hrs}$ at $4 \mathrm{oC}$ abolished all staining of Manduca AL tissue. E: Non-pre-adsorbed controls in which rabbit anti-GABA antibody was incubated in parallel under identical conditions with the exception of GABA resulted in strong immunolabeling. F: Pre-adsorption of the rabbit anti-MIP antiserum by incubating with $1 \mathrm{mg} / \mathrm{mL}$ BSA and 10:1 synthetic MIPVI (AWSALHGAWA): rabbit anti-MIP antibody for $24 \mathrm{hrs}$ at $4 \mathrm{oC}$ abolished all staining of Manduca AL tissue. G: Nonpre-adsorbed controls in which rabbit anti-MIP antibody was incubated in parallel under identical conditions with the exception of the synthetic MIPVI resulted in strong immunolabeling. $\mathbf{H}$ : Pre-adsorption of the rabbit anti-TKK antiserum by incubating with $1 \mathrm{mg} / \mathrm{mL}$ BSA and 10:1 synthetic TKKI (RAPMGFMGVR): rabbit anti-TKK antibody for $24 \mathrm{hrs}$ at $4 \mathrm{oC}$ abolished all staining of Manduca AL tissue. I: Non-pre-adsorbed controls in which rabbit anti-TKK antibody was incubated in parallel under identical conditions with the exception of the synthetic TKKI resulted in strong immunolabeling. Scale bar $=100 \mathrm{um}$. 

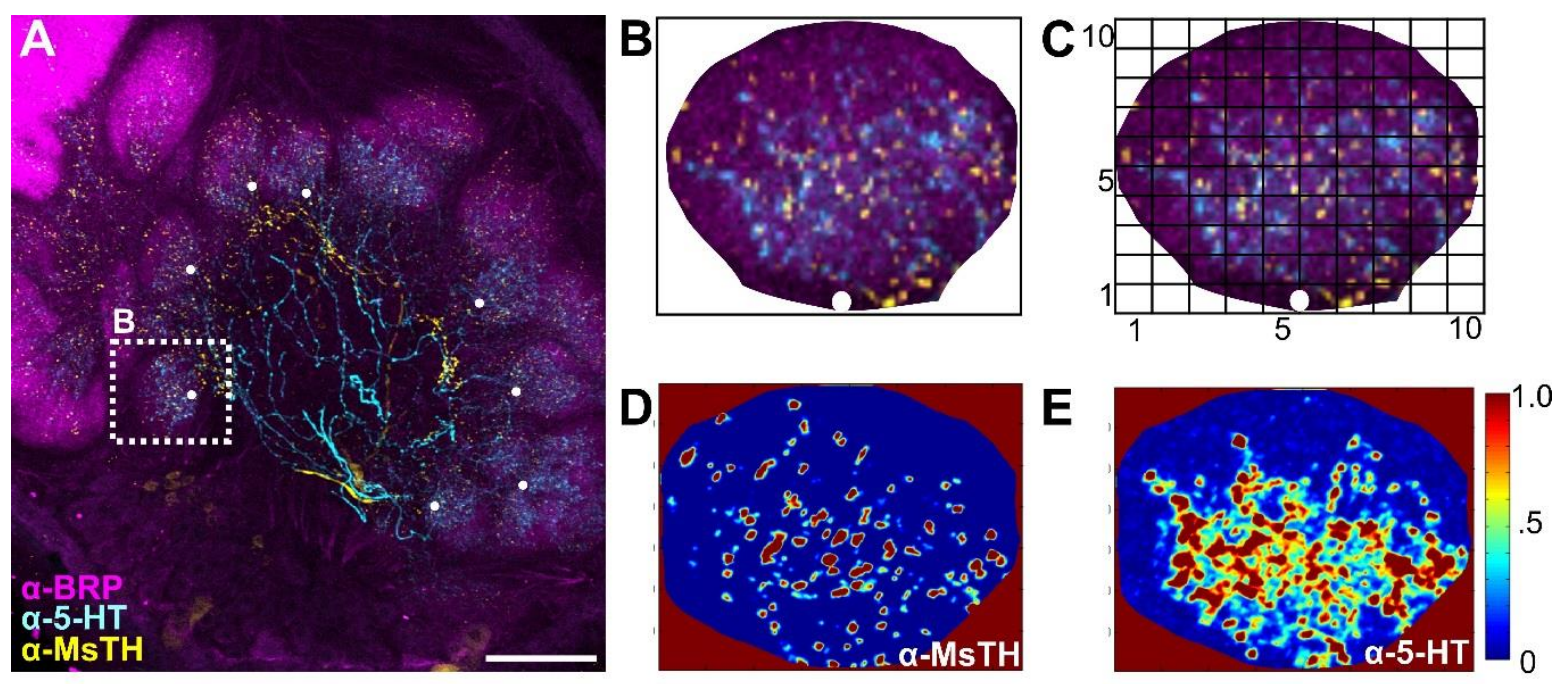

Figure 2. Depiction of Intensity Distribution Analysis.

A: Example .tif stack of an AL scan with glomeruli marked with a white dot at their most proximal, central point. B: Cropped glomerulus, marked in Fig. 2A, oriented such that the white dot is at the " 6 o'clock" position to ensure that horizontal bin 1 always corresponds to the most proximal end of the glomerulus and horizontal bin 10 to the most distal. A white bordered square was placed with edges flush to the cropped glomerulus. The square is shown as black here to visualize concept. C: The area innervated and the density of pixels for each modulator was determined across a 10x10 matrix using a custom MatLAB script. Horizontal Bin 1 is most proximal, while horizontal bin 10 is most distal. $\mathbf{D}$ : MatLAB determined intensity values for MsTH-ir. E: MatLAB determined intensity values for 5 -HT-ir. Scale bar = $100 \mathrm{um}$. 

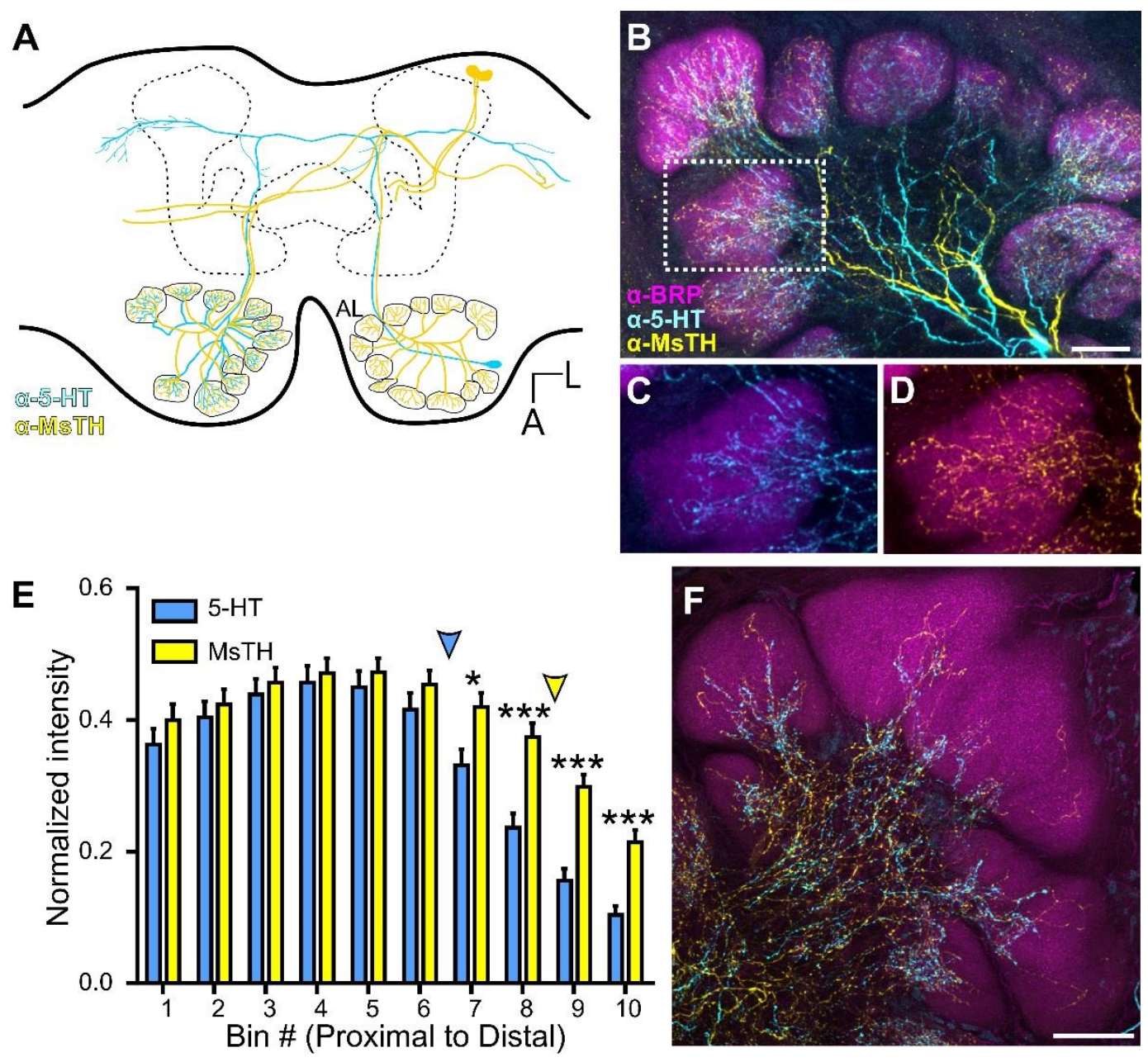

Figure 3. Convergence of 5-HT-ir and MsTH-ir in AL glomeruli. A: Schematic of one pair of DAAR neurons (yellow) and a single CSD (blue) neuron within the brain of Manduca. The CSD cell bodies are located in the lateral cell cluster, while the DAAR cell bodies are located in the dorsal protocerebrum. Each AL is innervated by one CSD neuron which projects to the contralateral $A L$ and innervates all glomeruli. Four DAAR neurons (one pair on each side of the brain) project to both the ipsilateral and contralateral ALs innervating all glomeruli. B: 5$\mathrm{HT}$-ir (cyan) is densely distributed near the proximal region of glomeruli while MsTH-ir (yellow) is distributed sparsely throughout glomeruli. Glomerular boundaries are delineated by BRP-ir (magenta). C, D: Inset from B depicting individual channels with $5 \mathrm{HT}$-ir/BRP-ir and MsTH-ir/BRP-ir respectively. E:

Glomerular distribution of 5-HT-ir and MsTH-ir along the proximal to distal axis of isomorphic glomeruli. MsTH-ir extends farther distally than 5-HT (Multiple t-tests; $t=2.76$ for bin7, $t=4.55$ for bin $8, t=5.44$ for bin $9, t=4.68$ for bin 10 ; $d f=18$; $p=0.012$ for bin $7 p<0.0001$ for bin $8-10 ; n=100$ ). The distribution of each modulator began to significantly reduce in intensity along the proximal to distal axis at different points. For 5-HT, intensity values began to significantly decrease at bin 6 (1-way ANOVA; $p<0.01, d f=9, n=10)$, while for MsTH, the intensity values don't begin to significantly decrease until bin 9 (1-way ANOVA; $p<0.05$, df $=9$, $n=10$ ). Colored arrowheads denote where the intensity of each modulator begins to significantly reduce. F: Macroglomerular complex (MGC) demonstrating sparse innervation of both 5-HT-ir and MsTH-ir. All scale bars $=50 \mathrm{um}$. 

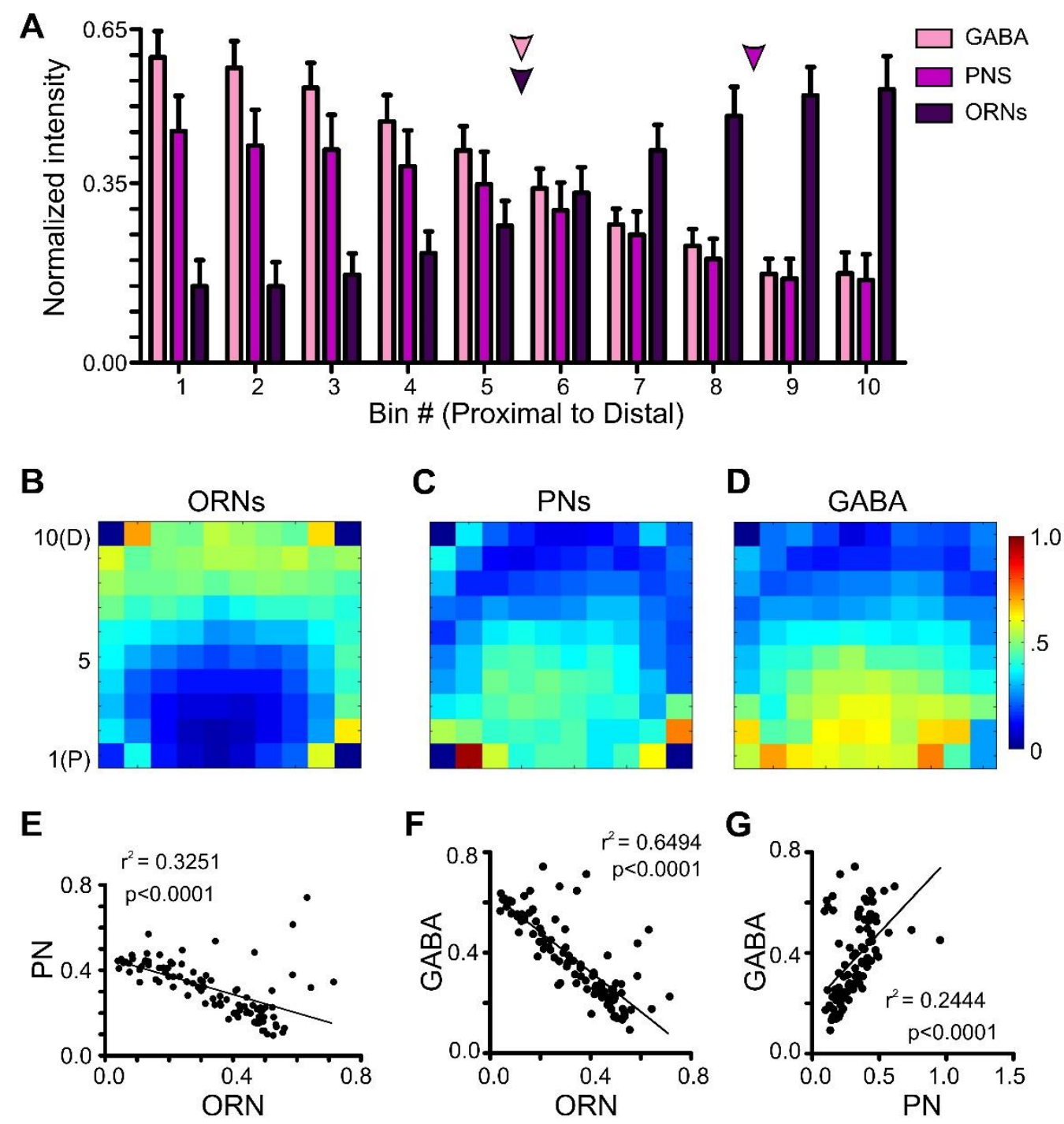

Figure 4. The anatomical boundaries of ORNs, PNs and GABA-ir. A: Label intensity for ORNs,, PNs and GABA-ir along the proximal (bin 1) to distal (bin10) axis of a glomerulus. ORN intensity values began to significantly decrease relative to the most distal bin at bin 6 (1-way ANOVA; $p<0.05, d f=9, n=10)$. PNs intensity values began to significantly decrease relative to the most proximal bin at bin 9 (1way ANOVA; $p<0.05, d=9, n=10$ ). GABA intensity values began to significantly decrease relative to the most proximal bin at bin 5 (1-way ANOVA; $p<0.01$, df $=9$, $n=10$ ). Colored arrows denote where the intensity of each neuronal type begins to significantly reduce. B-D: Averaged $10 \times 10$ intensity plots for each neuron type (ORN, PN and GABA-ir, respectively. $n=10$ each). Blue denotes low intensity values, while red denotes high intensity values. B: 10x10 intensity plot for ORNs. C: 10x10 intensity plot for PNs. D: 10x10 intensity plot for GABA-ir. E-G: Linear regressions of the average intensities across the $10 \times 10$ intensity plots of ORNs, PNs and GABA-ir. E: The innervation patterns of ORNs and PNs display a negative relationship ( $\mathrm{r} 2=0.3251, \mathrm{p}<0.0001, \mathrm{n}=10$ glomeruli, 5 animals). $\mathrm{F}$ : ORNs and GABA display a negative relationship $(r 2=0.6494, p<0.0001, n=10$ glomeruli, 5 animals). G: The innervation patterns of PNs and GABA display a positive relationship ( $r 2=0.2444, p<0.0001, n=10$ glomeruli, 5 animals). 

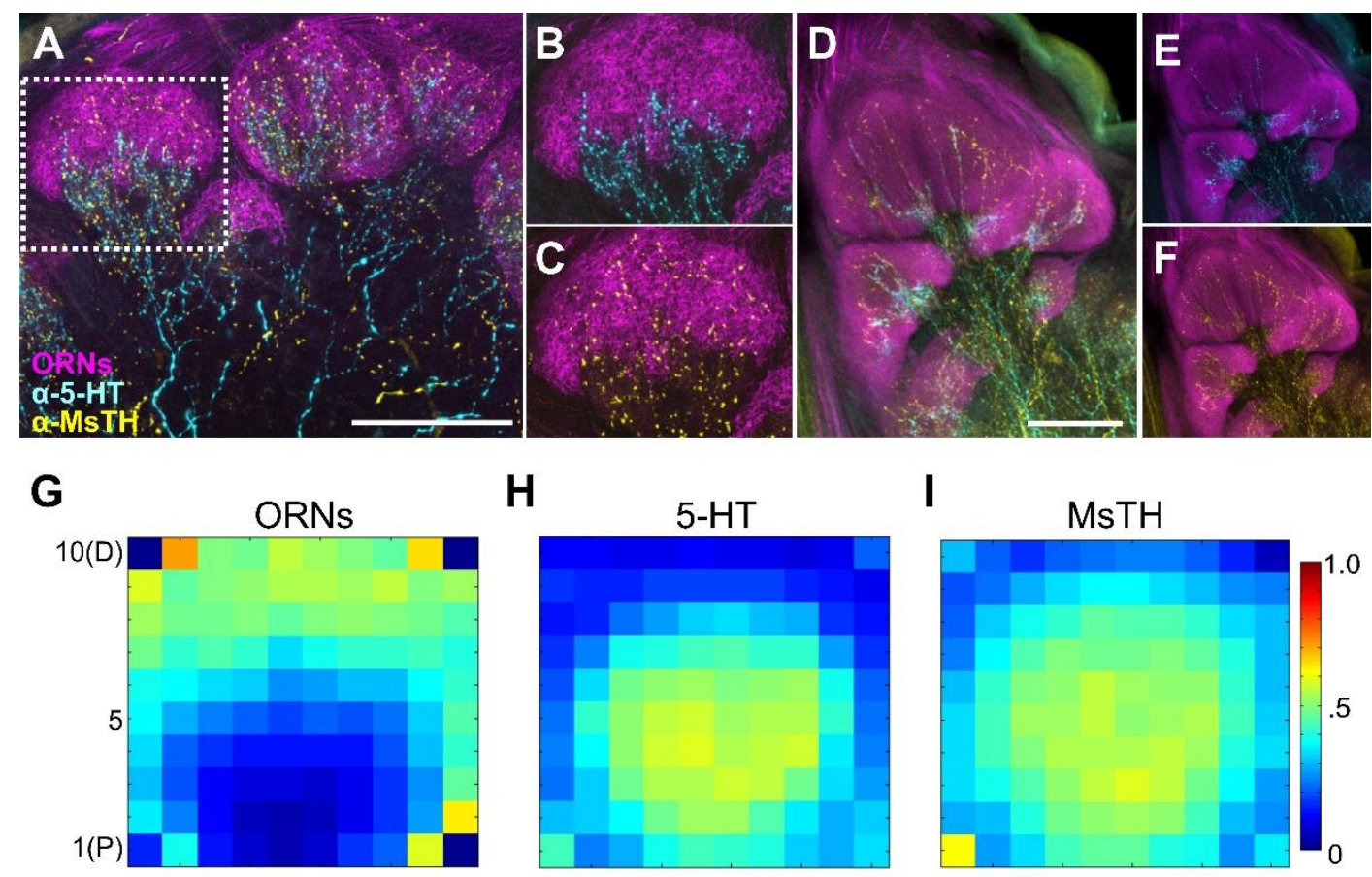

H
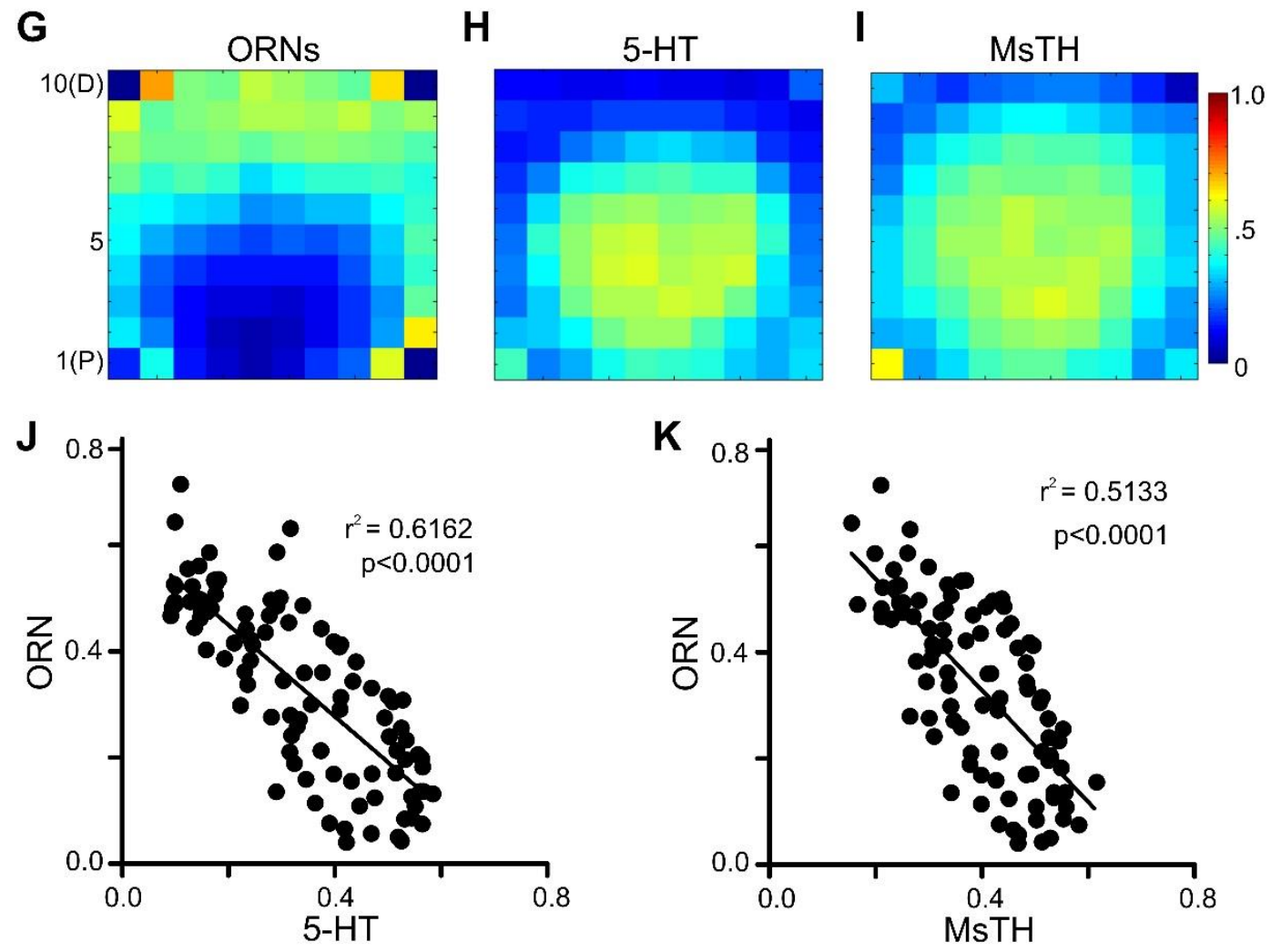

Figure 5. Processes of the CSD and DAAR neurons innervate distinct glomerular zones with relation to ORNs. A-C: MsTH-ir (yellow) overlap with ORN axons (magenta) at the distal region of the glomerulus while 5-HT-ir (cyan) is constrained to proximal glomerular regions. B, C: Inset from A showing 5HT and MsTH branching, respectively, with relation to ORN axons in the glomerulus. D-F: MGC innervation by the CSD and DAAR neurons relative to ORN axons. All scale bars $=50 \mathrm{um}$. G: $10 \times 10$ intensity plot for ORNs. H: 10x10 intensity plot for 5-HT-ir. I: 10x10 intensity plot for MsTH-ir. J-K: Linear regressions of the average intensities across the $10 \times 10$ intensity plots of ORNs, 5-HT-ir and MsTH-ir. J: 5$\mathrm{HT}$-ir and ORNs display a negative relationship $(\mathrm{r} 2=0.6162, \mathrm{p}<0.0001) . \mathrm{K}$ : ORNs and $\mathrm{MsTH}$-ir display a negative relationship $(\mathrm{r} 2=0.5133, \mathrm{p}<0.0001)$. 


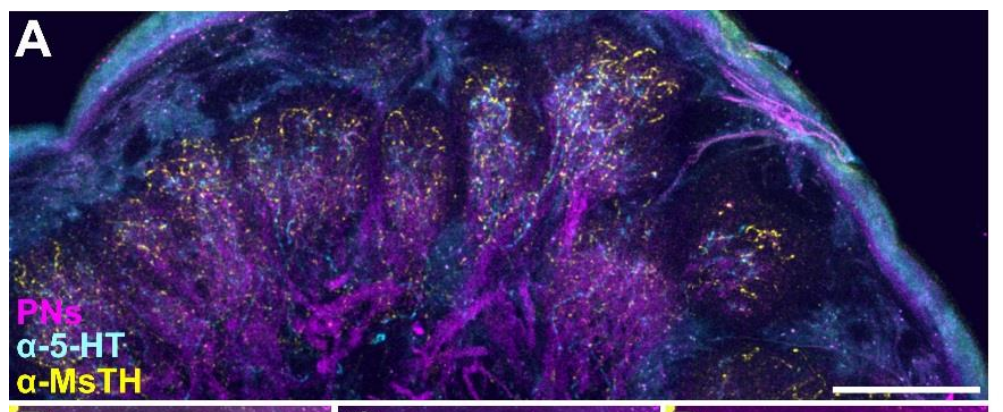

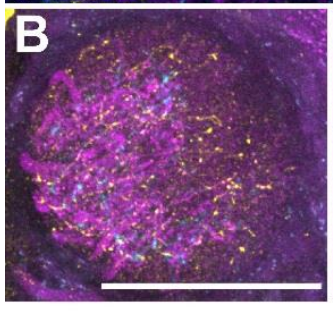

E

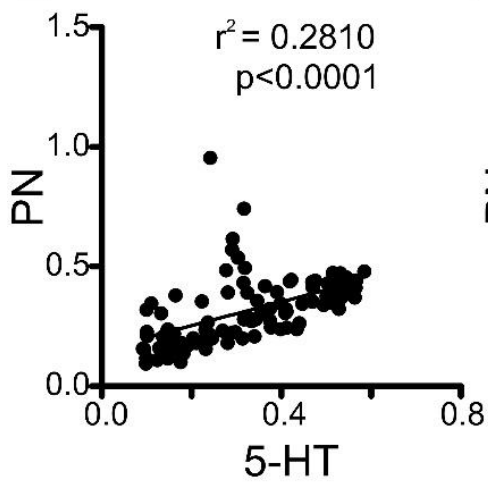

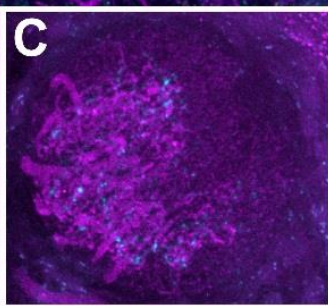

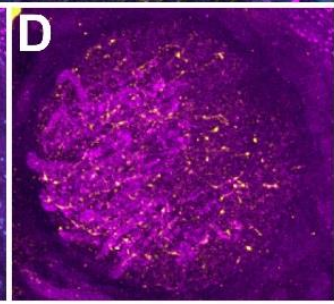

$\mathbf{F}$

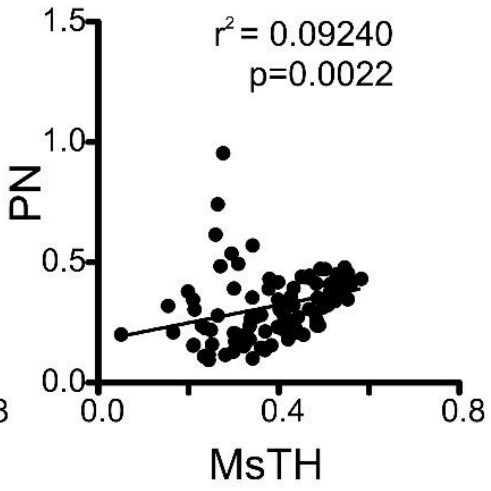

Figure 6. CSD and DAAR neuron processes overlap extensively with the processes of PNs.

A: The distribution of 5-HT-ir (cyan) and MsTH-ir (yellow) in isomorphic glomeruli relative to retrogradely filled PNs (magenta). B-D: High magnification examples of a glomerulus in which PNs, CSD and DAAR neurons have been labeled. B: PNs, 5-HT-ir and MsTH-ir depicted. C: PNs and 5-HT-ir depicted. D: PNs and MsTH-ir depicted. 5-HT-ir has nearly complete overlap with PNs, while MsTH-ir overlaps with PNs and extends distally. All scale bars $=50$ um. E-F: Linear regressions of the average intensities across the 10x10 intensity plots of PNs, 5-HT-ir and MsTH-ir. E: Linear regression $5-\mathrm{HT}$-ir and $\mathrm{PNs}$ display a positive relationship $(r 2=0.2810$, $\mathrm{p}<0.0001)$. F: PNs and MsTH-ir display a positive relationship $(r 2=0.09240, p=0.0022)$. 

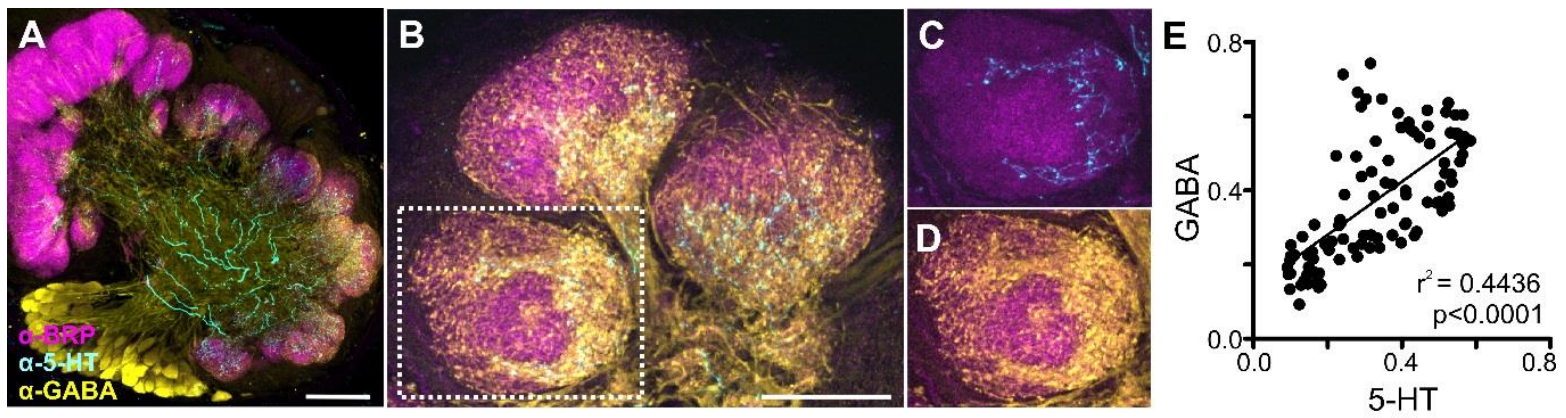

Figure 7. CSD processes overlap extensively with processes of GABA-ir LNs and PNs

A: AL immunolabeled for 5-HT (cyan), GABA (yellow) and BRP (magenta). Scale bar $=100 \mathrm{um}$. B:

Distribution of 5-HT-ir and GABA-ir in isomorphic glomeruli. Scale bar $=50 \mathrm{um}$. C-D: Inset from B showing relative distribution of 5-HT-ir and GABA-ir within isomorphic glomeruli. C: $5-\mathrm{HT}$ overlaps extensively with region occupied by the dense GABA-ir branching. D: GABA-ir extends throughout with denser processes at the proximal region and sparser innervation distally. $E$ : Linear regression of the average intensities across the $10 \times 10$ intensity plots of $5-\mathrm{HT}$-ir and GABA-ir displaying a positive relationship $(r 2=0.4436, p<0.0001)$. 

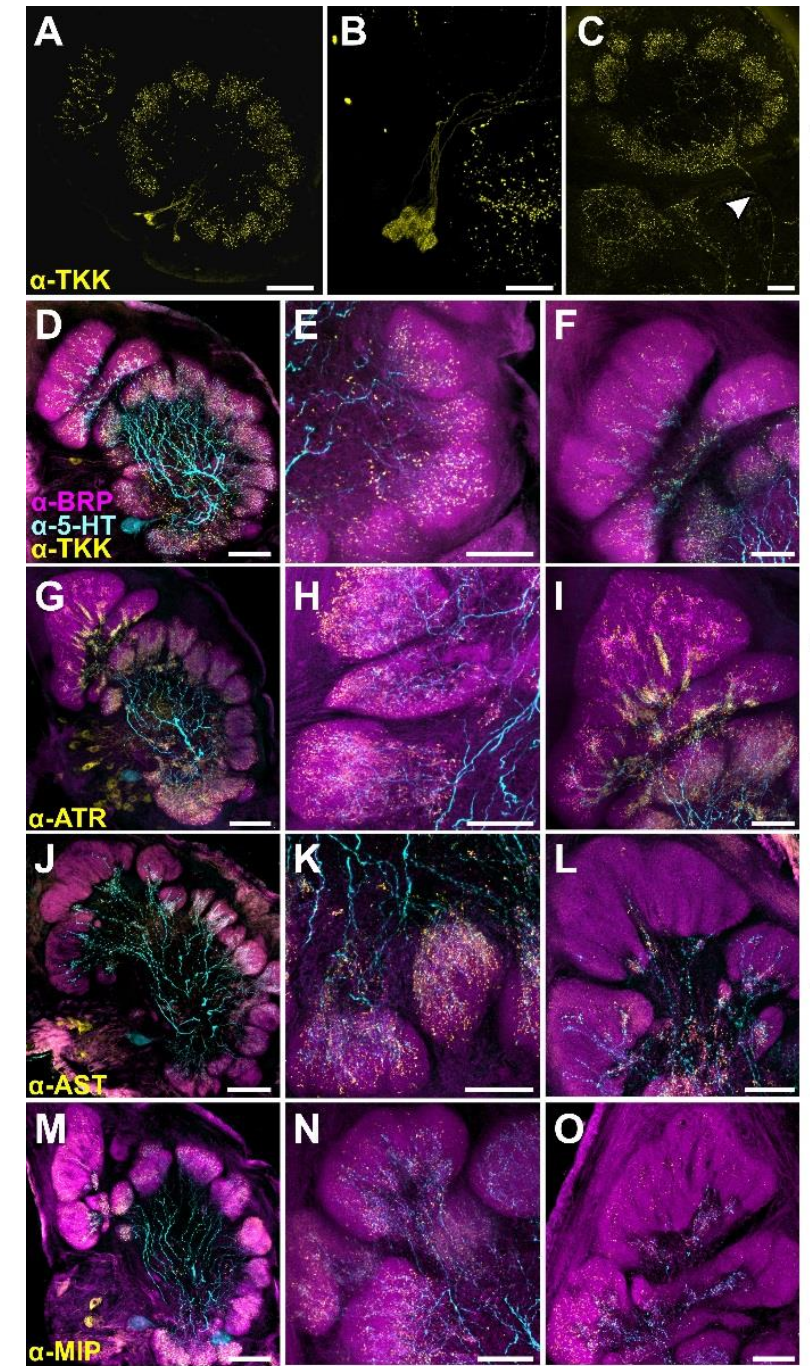

Figure 8: Distribution of processes from peptidergic neurons and the CSD neurons. A: Tachykinin (TKK) labeling in the AL. Each AL features $\sim 9$ TKK-ir cell bodies which in sum innervate all glomeruli B: High resolution scan of TKK-ir cell bodies in the lateral cell cluster. C: TKK-ir process leaving the AL (denoted by white arrow). While most TKK-ir bodies appear to be LNs according to their morphology, the process leaves the AL via the mALT, suggesting that TKK-ir cells may be PNs or centrifugal neurons. D-O: Glomeruli stained for various neuropeptides (yellow), 5-HT (cyan) and BRP (magenta). Left column features the $A L$ (scale bars $=100 \mathrm{um}$ ), the middle column features a representative isomorphic glomerulus and the right column features a representative MGC (scale bars $=50$ um). D-E: TKK-ir extends father distally than $5 \mathrm{HT}$ ir in isomorphic glomeruli. F: TKK-ir extends farther distally in the MGC than 5-HT-ir. G-I: Dense processes of ATR-ir LNs occur in isomorphic glomeruli and extend farther distally than 5HT-ir. I: Allatotropin (ATR) processes in the MGC extend farther distally than 5-HT-ir. ATR-ir processes are dense proximally but more spare as they extend distally in the MGC. J-L: Allatostatin (AST) processes extend farther distally in the glomeruli than $5 \mathrm{HT}$-ir and are uniform in density throughout. L: AST-ir sparsely innervates the MGC and remains proximal with 5-HT-ir. M-O:

Myoinhibitory peptide (MIP) processes extend much farther distally than 5-HT-ir in the isomorphic glomeruli and are uniform in density throughout. O: MIP-ir extends farther distally than $5 \mathrm{HT}$-ir within the MGC. 


\section{CHAPTER 3}

Serotonin and Dopamine Modulate Olfactory Coding via Distinct Mechanisms 


\begin{abstract}
:
Animals constantly adjust sensory processing to meet the ongoing demands of a dynamic internal and external environment. In the olfactory system, extrinsic modulatory neurons adjust odor coding within the context of ongoing activity in other brain regions, yet the consequences of integrating multiple modulatory inputs are not well understood. The antennal lobe (AL; the first olfactory neuropil) of the moth, Manduca sexta is innervated by widely projecting serotonergic and dopaminergic extrinsic neurons whose processes converge in common areas within glomeruli. Therefore, single AL neurons may be simultaneously influenced by both modulators. Using extracellular tetrode recordings of AL neurons, we asked 1) Do both modulators affect the same neurons? 2) How variable are the effects of a single modulator? 3) Do both modulators target the same features of odor-evoked responses? Our results demonstrate that 5-HT and DA alter olfactory processing by targetting both distinct and common phases of odor-evoked responses. While both 5-HT and DA converge on many of the same AL neurons, the individual effects of each modulator can diverge by targeting distinct phases of odor-evoked responses. We find that most projection neurons (PNs) integrate the influence of both 5-HT and DA, while a high proportion of putative local interneurons (LNs) were selectively modulated by 5-HT alone (although a small subset of LNs was affected by both modulators). The individual effects of 5HT and DA are variable as within both LNs and PNs, each modulator increased the odor-evoked firing rate of subsets of neurons while decreasing the odor-evoked firing rates of other neurons. Additionally, when both modulators were applied together, many of the individual effects of each modulator were attenuated. This suggests that while both 5-HT and DA can converge onto the same neuron, their effects on second-messengers may diverge causing a non-linear change to odor-evoked responses. Altogether, our results suggest that DA modulates response timing of PNs by targeting intrinsic inhibitory phases of PNs, while 5-HT alone may alter PN sensitivity at low odor concentrations by increasing PN response duration and altering the balance of lateral input via LNs. Finally, this work provides a mechanistic basis by which a sensory system integrates multiple modulators within single neurons to mediate network-wide changes in response to an ecologically relevant stimulus.
\end{abstract}




\section{Introduction:}

Every neural network is influenced by a dynamic cocktail of neuromodulators that are integrated by individual neurons to mediate network-wide, context specific changes in output. While this challenge is ubiquitous across species, brain region, and neuron type, little is known about how individual functional neuron types integrate or segregate the simultaneous influence of multiple modulators. In motor systems, the physiological consequences of multiple modulators have been studied at the level of single neurons (Gutierrez and Marder 2014; Marder et al., 2014), and populations of neurons (Blitz et al., 1999; Brezina et al., 1996; Mesce et al., 2001; Thirumalai and Marder, 2002; Beliez et al., 2014). Key findings from motor systems demonstrate that integration of multiple modulators onto single neurons can alter network processing by a variety of mechanisms (reviewed in Marder, 2012; Nadim and Bucher, 2014). For example, while it's tempting to assume that the individual effects of modulators may integrate linearly, modulators that affect neurons in a similar fashion when applied alone, have drastically different and non-linear effects when combined (Swensen and Marder, 2000; Li et al., 2018). Moreover, two modulators could converge or diverge by targeting distinct (Thirumalai and Marder, 2002) or shared targets (Swensen and Marder, 2000) within the network. Modulators could also molecularly converge (or diverge) onto the same (or opposing) second messenger cascades, resulting in additive, subtractive, or non-linear effects on responses (Brezina et al., 1996; Mesce et al., 2001). Finally, modulators can alter synaptic input to a given neuron within the network, thereby altering the balance of excitation or inhibition that neuron experiences (Katz and Frost, 1995). Combinations of these mechanisms likely occur simultaneously within single neurons to mediate network wide changes in output. However, the details of modulatory integration are lacking in sensory systems as most studies have focused on determining the effects of only one neuromodulator at a time. Ultimately, to understand realistic sensory network function, it is critical to determine the network consequences of integrating multiple neuromodulatory inputs in single neurons.

Here, we take advantage of the wealth of anatomical and physiological information on the individual effects of two modulators, serotonin (5-HT) and dopamine (DA), within the olfactory system of the moth, M. sexta. In the insect antennal lobe (AL: the first olfactory neuropil, analogous to the vertebrate olfactory bulb), odorants bind to chemosensory proteins expressed by 
input neurons called olfactory receptor neurons (ORNs). Within a glomerulus, ORNs synapse upon output neurons called projection neurons (PNs). Finally, a diverse population of local interneurons (LNs) (Chou et al., 2010; Reisenman et al., 2011; Seki et al., 2010; Lizbinski et al., 2018; Christensen et al., 1993, Lei et al., 2002) refines the input/output relationship of ORNs and PNs. In $M$. sexta, the AL is also innervated by centrifugal neurons releasing serotonin (5-HT), and dopamine (DA) which act as extrinsic modulators to alter olfactory processing in the context of waking state and aversion (Dacks et al., 2008; Dacks et al., 2012; Kent et al., 1987; Kloppenburg et al., 1999). Within each glomerulus, both 5-HT and DA overlap with the processes of PNs and LNs, suggesting that these two functional neuron types may integrate the influence of both modulators (Lizbinski et al., 2016). Previous studies have shown that both 5-HT and DA generally enhance odor-evoked responses across populations of AL units (Dacks et al., 2008; Dacks et al., 2012). Futhermore, both modulators can alter different potassium conductances, thereby changing a neuron's excitability (Mercer et al., 1996; Perk et al., 1995). Thus, while the individual physiological effects on odor-processing of both 5-HT and DA have been previously studied, it is unknown whether AL neurons integrate the influence of both modulators, the degree to which the effects of single modulators are variable, and finally if any integrated effects of these modulators are linear.

It is also unclear whether both modulators alter the same features of odor-evoked responses of AL neurons. Here we find that serotonin (5-HT) alone increases response duration of PNs and can increase or decrease odor-evoked firing rate depending on the PN, but does not affect a post-excitation inhibitory phase intrinsic to PNs ("I2"). Dopamine (DA) alone also can increase or decrease odor-evoked firing rate depending on the PN, however it significantly shortens I2, allowing PNs to recover to baseline quicker. This suggests that while both 5-HT and DA affect odor-evoked excitation, they affect different temporal features of the overall odorevoked responses of PNs. In combination, 5-HT and DA can increase or decrease odor evoked firing rate depending on the neuron, however the 5-HT mediated increase in response duration is lost, suggesting that DA masks the 5-HT mediated increase in response duration. Frequently, DA appears to mask the effects of 5-HT on specific coding features when both modulators are integrated in a single neuron. In LNs, both modulators alone can either increase or decrease postinhibitory rebound excitation, however only 5-HT affected the length of odor-evoked inhibition in LNs. This suggests that while both modulators affect rebound excitation, they likely affect this 
response feature via distinct mechanisms as both strength of inhibition and channel opening probability affect rebound. Overall, we find that both PNs and LNs integrate the influence of both 5-HT and DA, and the effects of each modulator individually are highly variable on an odor-concentration and cell-by-cell basis (either increasing or decreasing odor-evoked firing rate in subsets of AL neurons). Finally, while each modulator has similar effects on odor-evoked responses when applied alone, their integrated effects suggest each modulator can affect the same feature of olfactory coding by potentially targeting distinct cellular and synaptic mechanisms.

\section{Materials and Methods:}

Animals: M. sexta was raised at West Virginia University on a 16:8 reverse light:dark cycle in house as previously described (Bell et al., 1976; Daly et al., 2013). All moths were naïve, unmated adults between 2-5 days post-eclosion. 21 moths were used for the experiments assessing the effects of both modulators for a total of 47 neurons, and 9 moths were used for control experiments for a total of 37 neurons.

\section{Extracellular multichannel electrophysiology:}

Using extracellular multichannel electrophysiological recording, the responses of individual neurons to a stimulus set were compared across pharmacological treatments of 5-HT and DA individually, as well as 5-HT and DA together. For recording, moths were fixed with wax into tubes, a window cut into their head capsule to expose the ALs and physiological saline superfused over the brain (Figure 1A). Sixteen-channel extracellular electrode arrays (Neuronexus) were inserted into the AL allowing the recording of multiple AL neurons simultaneously. Extracellular activity was acquired with a P22-32 32-channel pre-amp from Tucker Davis Technologies (TDT) and an RZ5D Bioamplifier Processor from TDT. The thresholds to capture waveforms were set individually and independently for each channel. Separate sets of animals were used to examine sensitivity as well as control experiments for a

total of 83 individual units. Dilutions of $10^{-4}, 10^{-3}, 10^{-2}$, and $10^{-1}$ synthetic Datura wrightii blend odorant (M. sexta's host plant) were used to determine how 5-HT and DA affect sensitivity to odors (Riffel et al 2009; Riffell et al 2008). Datura wrightii is the host plant of M. sexta and contains individual chemical components that span multiple functional groups, thus activating a 
large number of glomeruli. Odor was prepared by mixing individual components of the floral head space with pure mineral oil (Riffell et al., 2009; Riffell et al., 2008). Odor was puffed onto the antennae from lowest to highest concentration to evoke a response from AL neurons. 200ms pulses of each odor was repeated 10 times with an inter-pulse interval of 10 seconds. The stimulus set was repeated while either control saline or neuromodulators were superfused $\left(10^{-4} \mathrm{M}\right.$ 5-HT and $\left.10^{-5} \mathrm{M} \mathrm{DA}\right)$. Neuromodulator concentrations are based on dose-response curves performed in previous publications (Dacks et al., 2008; Dacks et al., 2012). The order in which 5-HT or DA alone were applied was randomized between each experiment to avoid any bias based on order of application, however the application of both modulators simultaneously was always last, followed by the final control saline wash. Each neuromodulator solution was prepared fresh each and immediately before recording, applied and washed out with $M$. sexta physiological saline (Christensen and Hildebrand, 1987) for 10 minutes. Spontaneous activity was recorded for 1 minute during each treatment before odor stimuli to determine the stability of the neuron across treatments and assess whether each modulator alters spontaneous activity (Figure 1B).

Spike sorting: The spike timing of individual neurons were assigned based on waveform shape by Offline Sorter v3.3.2 (Plexon Inc.). Non-automated cluster-cutting was performed by hand using principal components analysis (PCA) and specific waveform parameters for each individual channel within a tetrode such as peak/valley ratio to cluster neurons in 3-D space. Contamination due to short ISIs was kept below $1 \%$ for all units to avoid assigning multiple waveforms from disparate units to the same unit. Cross-correlograms were used to compare units recorded within the same experiment to avoid overcutting.

Data analysis: The firing rate of each neuron in response to the stimulus set was determined by creating perievent rasters for each unit in response to all odor concentrations using NeuroExplorer v4 (Plexon Inc.). M. sexta PNs have 3 easily identifiable phases of response, brief inhibition (I1), excitation (E) and a post excitation inhibitory phase of variable length (I2) that local interneurons do not possess (Waldrop et al 1987; Christensen et al., 1998; Heinbockel et al., 1999). Therefore, we can confirm with a high-degree of certainty that a neuron is a PN based on the presence of I1, E, and I2, but the absence of these phases is not enough to definitively confirm that we recorded an LN. Therefore, neurons lacking the I1, E, and I2 phases were 
categorized as 'putative' LNs, and we can't rule out that some may be PNs with non-traditional odor-evoked responses. Using these criteria, units were assigned to two groups, PNs and putative LNs. Custom Matlab scripts were used to extract the length of I1, E and I2 for all PNs. Scripts also calculated the response integral (defined as the area under the curve during the excitation phase) for every neuron. For LNs, the script extracted either the length of inhibition, the length of excitation, or the length of rebound excitation of each neuron (depending on the type of response produced), and then determined the response integral for any excitatory phase. The firing rate of each neuron was determined by analyzing the number of odor-evoked spikes elicited by each pulse of odor during the excitation phase of each neuron type.

The effects of modulator treatments were analyzed for all individual units. For all measures, responses were normalized within modulator treatment (the saline wash immediately preceding and following each individual modulator treatment). Stability of units across treatments was determined using both the peak/valley ratio of the waveform, and the mean interspike interval (ISI) was calculated for every neuron's spontaneous activity across treatment to quantify its firing rate. Each modulator treatment was compared to the saline wash immediately preceding and following it. Responses were tested for normality using the D’Agostino-Pearson Omnibus Test, and if normal a 1-way ANOVA with Bonferroni correction run, if not-normally distributed a Friedman's test with a Dunn's multiple comparison test to test whether the neuron's spontaneous activity differed across saline and modulator treatment. ISI of spontaneous activity of all units was not significantly different across all washes (1-way ANOVA, Friedman test, Dunn's multiple comparison test, all treatments not significantly different (n.s.) than each other; Supp. Figure 1A). Control experiments were also performed to assess the stability of odor-evoked activity across time. In these experiments, AL neurons were recorded in only physiological saline bath application across 3 'treatment' washes. Response averages to all concentrations of odor were not significantly different across all saline washes (1way ANOVA, Friedman test, Dunn's multiple comparison test, all treatments not significantly different (n.s.) than each other), demonstrating that this technique allows for stable recording of AL units over time (Supp. Figure 1B). There was a significant difference between Saline 2 and Saline 3 at [1:100] (1-way ANOVA, Friedman test, Dunn's multiple comparison, p<0.05; Supp. Figure 1B), therefore for all measures, responses that compare 5-HT, DA or 5-HT+DA to control conditions were compared to the saline wash immediately preceding and following the 
modulator wash. Dose response curves for sensitivity experiments were calculated from the neuron's response integral to increasing odor concentrations. The slope of this curve was calculated to determine if modulators affected the gain of individual neurons. The duration of three phases of PN responses, I1, E (excitation phase of the response) and I2, was compared across modulator treatments to determine the modulators affect the temporal fidelity with which PNs can encode odor information. This analysis was performed using custom Matlab scripts and GraphPad Prism v6 (GraphPad Software, Inc.) for all other statistical analyses.

\section{Results:}

Previous work on ensembles of insect AL neurons (Riffell et al 2013; Dacks et al 2008; Dacks et al., 2012) did not attempt to distinguish the effects of modulation on each functionally distinct class of principal AL neurons. Here, we take a unique approach to assess the effects of modulators on distinct functional classes of AL neurons. After spike sorting, we took advantage of characteristic differences in odor-evoked activity of two principal neuron types to sort our units into two groups: PNs and putative LNs. In M. sexta projection neurons have 3 easily identifiable phases of odor-evoked response, brief inhibition (I1), excitation (E) and a post excitation inhibitory phase of variable length (I2) that LNs do not possess (Waldrop et al 1987; Christensen et al., 1998; Heinbockel et al., 1999; Figure 1C). I1 is mediated by $\mathrm{Cl}^{-}$and is abolished by GABAa antagonists. However, the mechanism underlying underlying I 2 is not fully understood. It is likely due to either intrinsic properties of the PNs themselves or GABAb mediated based on a few studies: 1) Bicuculine, a GABAa antagonist abolishes I2, however bicuculine has also been shown to affect $\mathrm{K}+$ channels that mediate after-hyperpolarization in addition to its effects on GABAa receptors (Khawaled et al., 1999). Furthermore, the reversal potential for $\mathrm{I} 2$ is more negative than that of I1 which demonstrates that I 2 is likely mediated by $\mathrm{K}+$ conductance and not $\mathrm{Cl}^{-}$(and therefore not GABAa mediated). Overall, I2 is likely due to intrinsic $\mathrm{K}^{+}$channels or GABAb mediated. (Waldrop et al., 1987; Christensen et al., 1998; Heinbockel et al., 1999). LNs do not produce I1 and I2 and fall into two categories 1) excited by odors and 2) inhibited by odors (Figure 1D). Although only PNs have been shown to robustly and consistently exhibit stereotyped I1 and I2 (Waldrop et al., 1987; Christensen et al., 1998; Heinbockel et al., 1999), the absence of these phases is not enough to definitively confirm a recorded neuron is an LN. Therefore, neurons lacking the I1, E, and I2 phases were categorized 
as 'putative' LNs, as we can't rule out that some neurons in this group may be PNs with nontraditional odor-evoked responses. Using these criteria, recorded units were assigned to two groups, PNs and putative LNs, and the effects of each modulator treatment were assessed based on this distinction.

\section{Most projection neurons integrate the influence of both 5-HT and DA}

The integrated effects of both modulators may follow three distinct possibilities, in which neurons could be affected by single, both or neither modulators. For example, there may be nonoverlapping populations of neurons selectively affected by 5-HT and DA, populations of neurons that can or cannot be affected by both modulators, and even completely overlapping populations simultaneously affected by 5-HT and DA. To determine where along this spectrum AL neurons lie, we first determined whether both modulators can affect the same functional neuron type, beginning with PNs. The largest proportion of total PNs (47\%) had their odor-evoked firing rate affected by both modulators (Figure 2A). Subsets of PNs were selectively modulated by 5-HT alone (18\%), DA (23\%) alone, and an even smaller subset were unaffected by either (12\%)

(Figure 2A). This suggests that most PNs integrate the influence of both 5-HT and DA.

\section{5-HT and DA exhibit variable effects across all projection neurons}

Past studies have shown that 5-HT and DA generally increase odor-evoked firing rate of AL neurons (Dacks et al., 2009; Dacks et al., 2012). However, they also found smaller subsets of neurons whose firing rate was decreased or unaffected by either modulator. We therefore asked, of the neurons affected by one or both modulators, how variable are the effects of each modulator on odor-evoked firing rate. The effects of 5-HT and DA were highly variable across the PNs recorded with 5-HT increasing and decreasing the firing rate of equal proportions of PNs, while DA decreased firing rate in a larger proportion of PNs (Figure 2B). The odor-evoked firing rate of some PNs was unaffected by either modulator (Figure 2B). Interestingly, the percentage of total PNs that had their odor-evoked firing altered by the simultaneous application of 5-HT and DA was not proportionally consistent with the number of PNs affected individually by either modulator (Figure 2B). This suggests that while 5-HT and DA may individually increase or decrease firing rate in the same neuron, their integrated effects may be non-linear, suggesting that each modulator likely affects opposing second-messengers or the synaptic input to that neuron has been altered by both modulators. For example, in some PNs, odor evoked 
firing rate was increased by both DA (1-way ANOVA, Friedman test, Dunn's multiple comparison, Saline 1 to DA: p<0.001; DA to Saline 2: p<0.0001; Figure 2C-D), and 5-HT (1way ANOVA, Friedman test, Dunn's multiple comparison, Saline 1 to 5-HT: $\mathrm{p}<0.0001$; 5-HT to Saline 2: $\mathrm{p}<0.0001$; Figure 2 E-F). In the same PN, the integrated effects of both 5-HT and DA still increased odor-evoked firing rate, but the effect was not a linear sum of the individual effects of the individual modulators (1-way ANOVA, Friedman test, Dunn's multiple comparison, Saline 1 to 5-HT+DA: p<0.0001; 5-HT+DA to Saline 2: n.s.; Figure 2G-H).

We then asked, were the effects of each modulator on response integral consistent across all odor concentrations? For instance, a modulator may only affect responses at certain points within the response range of a neuron, such that only responses at certain odor-concentrations are affected. We found that subsets of PNs had their response integral significantly increased by 5HT at the two lowest odor concentrations, (Kruskall-Wallis test, Dunn's multiple comparison test, [1:10,000], p<0.01, [1:1000], p<0.001, all other [odor] n.s.; Figure 3A). DA and 5-HT+DA did not significantly increase response integral in these PNs (Kruskall-Wallis test, Dunn's multiple comparison test, all [odor] n.s.; Figure 3A). This suggested that 5-HT may serve to modulate the sensitivity of those PNs by increasing the strength of response over time. In another subset of PNs, DA decreased response integral of PNs at low [odor] (Kruskall-Wallis test, Dunn's multiple comparison test, [1:1000], p<0.01, all other [odor] n.s.; Figure 3B). 5-HT+DA decreased this subset of PN response integrals at low [odor] (Kruskall-Wallis test, Dunn's multiple comparison test, [1:10,000], p<0.001, [1:1000], p<0.01, all other [odor] n.s.; Figure 3B). While there was a trend for 5-HT to decrease response integral in this subset of PNs, 5-HT did not significantly decrease response integral in these PNs (Kruskall-Wallis test, Dunn's multiple comparison test, all [odor] n.s.). While 5-HT significantly increased PN response integral in a sub-set of PNs, DA had its strongest effect by decreasing PN response integral at low odor concentrations. For PNs whose response integral was increased by either 5-HT, DA attenuates the 5-HT induced increase of response integral when both modulators are applied together. This suggests that the effect of DA on odor concentration dependent modulation may dominate when both modulators are integrated.

An efficient way for neurons to avoid saturation and maintain response range is by modulating the gain (Olsen and Wilson, 2008; Root et al., 2008; Martin et al., 2011; Wang, 
2012; Lizbinski and Dacks 2018). We therefore asked if either modulator affected the gain of PN responses by assessing the odor-evoked firing rate of control vs. modulator treatment across all odor concentrations. If the slope across odor-evoked responses to increasing odor concentrations differed between control and modulator treatment, it suggests that modulators may alter the response gain of PNs. While, there was a trend for DA to generally decrease odor-evoked firing rate, and for 5-HT to generally increase odor evoked firing rate across all PNs, there was no significant change to the slope for either DA (ANCOVA: $F=0.449194, \mathrm{DFn}=2, \mathrm{DFd}=174, \mathrm{P}=$ 0.6389; Figure 3C), 5-HT (ANCOVA: $\mathrm{F}=0.392765, \mathrm{DFn}=2, \mathrm{DFd}=186, \mathrm{P}=0.6757$; Figure 3D) or both modulators at any odor concentration (ANCOVA: $\mathrm{F}=2.34048, \mathrm{DFn}=2, \mathrm{DFd}=174, \mathrm{P}=$ 0.09931; Figure 3E). Therefore, when averaged across all PNs, there was no detectable change in gain by any modulator.

\section{5-HT and DA target distinct phases of odor-evoked responses of projection neurons}

Next we determined how variable the effects of each modulator were on specific phases of PN odor-evoked responses. PNs have a stereotyped brief inhibitory phase called I1 that is a GABAa mediated increase in $\mathrm{Cl}^{-}$conductance (Waldrop et al., 1987; Christensen et al., 1998; Heinbockel et al., 1999). This brief inhibition is due to polysynaptic, lateral inhibition by inhibitory LNs synapsing onto PNs (Waldrop et al., 1987; Christensen et al., 1998; Heinbockel et al., 1999). Changes to the I1 phase results in how long a PN exhibits an 'excitatory' odor evoked response, and therefore any modulatory effect on the I1 phase would have an effect on response timing. We determined whether this inhibitory phase is sensitive to 5-HT and DA modulation. The effects of either modulator could vary across two parameters: by odor concentration, as well as their effect on I1. We found that DA can significantly decreased the length of I1 at multiple odor concentrations (Kruskall-Wallis test, Dunn's multiple comparison test, [1:10,000], $\mathrm{p}<0.05$; [1:1000], n.s.; [1:100], p<0.05; [1:10], $\mathrm{p}<0.05$.; Figure 4A). 5-HT significantly decreased I1 length at one odor concentration (Kruskall-Wallis test, Dunn's multiple comparison test, [1:1000], p <0.05.; all other [odor] n.s.; Figure 4A). 5-HT also significantly increased I1 in a different subset of PNs at [1:1000] (Kruskall-Wallis test, Dunn's multiple comparison test, [1:1000], p<0.05.; all other [odor] n.s.; Figure 4B). The amount of inhibitory input a cell receives alters the amplitude of I1 (Heinbockel et al., 1999), suggesting that 5-HT likely alters the length of I1 by changing the amount of inhibitory drive onto PNs. The integrated effects of 
5-HT and DA increased the length of I1 at the highest and lowest odor concentrations (KruskallWallis test, Dunn's multiple comparison test, [1:10,000], $\mathrm{p}<0.05$.; [1:10], $\mathrm{p}<0.05$; all other [odor] n.s.; Figure 4B). This suggests that the combined effects of both modulators exhibit complex effects on GABAergic mediated lateral inhibition onto PNs. Overall, DA appears to modulate how quickly PNs respond to odors by decreasing the length of I1, while 5-HT had opposite effects on I1 depending on the PN and odor concentration. This suggests that this GABAa dependent form of inhibition is differentially sensitive to each modulator with DA playing a strong role in modulating response timing.

Due to the distinct effects of 5-HT and DA on the I1 phase of PNs, we asked whether both modulators differentially affect the post-excitation inhibitory phase of PNs called I2. I2 is likely an intrinsic property of PNs mediated by $\mathrm{K}^{+}$channels, causing delayed hyperpolarization that prevents PNs from exhibiting rapid succession of excitatory responses (Waldrop et al., 1987; Christensen et al., 1998; Heinbockel et al., 1999). Therefore modulating I2 affects the fidelity and timing by which PNs can encode the temporal dynamics of odor stimuli. We found that DA significantly decreases I2 at the highest odor concentration (1-way ANOVA, Bonferroni's multiple comparison test; [1:10], p<0.0001; all other [odor] n.s.; Figure 4C), while 5-HT alone (1-way ANOVA, Bonferroni's multiple comparison test; all [odor] n.s.; Figure 4D) and 5HT+DA (1-way ANOVA, Friedman test, Dunn's multiple comparison test; all [odor] n.s; Figure 4E), have no significant effect on I2. This further supports that DA modulates the odor-evoked response timing of PNs by decreasing the length of both inhibitory phases, I1 and I2.

Since we found that DA appears to modulate the timing of PN responses by decreasing the duration of I1 and I2, we asked whether either modulator affected another temporal feature of $\mathrm{PN}$ responses, response duration or the duration of the excitatory phase. We found that DA did not significantly alter response duration (1-way ANOVA, Bonferroni's multiple comparison test, n.s. across all [odor]; Figure 4D), but at the lowest odor concentration, 5-HT significantly increases the response duration of PNs (1-way ANOVA, Bonferroni's multiple comparison test, [1:10,000], p<0.05, all other [odor] n.s.; Figure 4E). 5-HT+DA together had no significant effect on response duration (1-way ANOVA, Bonferroni's multiple comparison test, n.s. across all [odor]; Figure 4H) This 5-HT mediated increase in response duration, much like the 5-HT mediated increase in response integral (Figure 3A) may serve to increase the sensitivity of AL 
neurons and increase detection thresholds at low concentrations of odor. The increase in response duration by 5-HT was lost when both modulators were applied together, suggesting that DA masks the effect of 5-HT on certain features of odor-evoked responses of PNs. Altogether, the DA mediated decrease of both I1 and I2 length (Figure 4A and 4C) suggests that DA may be modulating temporal features of PN responses. The 5-HT mediated increase in response duration (Figure 4G), response integral (Figure 3A), and general increase in firing rate across all odor concentrations (Figure 3D) suggests that 5-HT mediates changes in PN sensitivity by increasing the strength and duration of odor-evoked responses. Overall, these results demonstrate that 5-HT and DA target distinct phases of odor-evoked PN responses.

\section{Local interneurons integrate the influence of both 5-HT and DA, but the highest proportion are selectively modulated by 5-HT alone}

We then determined whether both 5-HT and DA could affect the same LN. Interestingly, the largest proportion of total putative LNs (37\%) had their odor-evoked firing rate affected by 5-HT alone (Figure 5A). Smaller subsets of LNs were selectively modulated by DA alone $(18 \%)$, both modulators (15\%), and a large subset were unaffected by either modulator (30\%) (Figure 5A). This suggests that while some LNs integrate the influence of both 5-HT and DA, more LNs are selectively modulated by 5-HT. This further suggests that 5-HT may be selectively modulating lateral input to other AL neurons. We then asked how variable the effects of 5-HT and DA on odor-evoked firing rate across the entire population of LNs. We did this across both response types of LNs; LNs exhibit either odor-evoked excitation, or odor-evoked inhibition followed by rebound excitation (Figure 1D). Across all LNs recorded, both 5-HT and DA had variable effects on firing rate, with each modulator capable of either increasing or decreasing odor-evoked firing rate during pure excitation or rebound excitation depending on the LN (Figure 5B). In some LNs, rebound excitation was increased by both DA (1-way ANOVA, Dunn's multiple comparison test, control saline to DA, $\mathrm{p}<0.0001$; DA to control saline, not significant (n.s.)); Figure 5C-D), and 5-HT (1-way ANOVA, Bonferroni's multiple comparison test, control saline to 5-HT p $<0.001 ; 5-\mathrm{HT}$ to control saline, $\mathrm{p}<0.0001$; Figure 5E-F). In the same LN, the integrated effects of both 5-HT and DA resulted in decreased rebound excitation, demonstrating that the integrated effects of both modulators can be subtractive (1-way ANOVA, Bonferroni's multiple comparison test, control saline to 5-HT+DA, p<0.0001; 5-HT+DA to 
control saline, n.s.; Figure 5G-H). Overall, both 5-HT and DA can have variable effects on LNs, either decreasing or increasing odor-evoked firing rate on a cell-by-cell basis. Furthermore, both modulators increase rebound excitation, demonstrating that similar to PNs, 5-HT and DA can target the same features of odor-evoked responses.

\section{Only 5-HT affects the length of odor-evoked inhibition in local interneurons}

Since 5-HT modulated the odor-evoked firing rate of the highest proportion of putative LNs, we asked whether 5-HT exhibits any distinct effects on odor-evoked inhibition in LNs. We found that 5-HT alone significantly decreases odor-evoked inhibition in LNs. At [1:1000] and [1:10], 5-HT significantly decreased the length of odor-evoked inhibition in a subset of PNs (Kruskill-Wallis test, Dunn's multiple comparison test, [1:1000], $\mathrm{p}<0.01$; $[1: 10], \mathrm{p}<0.0001$; all

other [odor] n.s.; Figure 6A-B). This effect could be due to either direct serotonergic modulation of LNs, or dis-inhibition due to decreased lateral LN-LN inhibition due to polysynaptic interactions. DA did not significantly alter odor-evoked inhibition (Kruskill-Wallis test, Dunn's multiple comparison test, all [odor] n.s.;Figure 6C-D), and the 5-HT mediated decrease in inhibition is lost when both modulators are applied simultaneously Kruskill-Wallis test, Dunn's multiple comparison test, all [odor] n.s.;Figure 6E-F). Overall, 5-HT alone decreases odorevoked inhibition in LNs, which is attenuated by DA when both modulators are applied together. This demonstrates that 5-HT and DA can also modulate distinct phases of odor-evoked responses.

\section{Discussion:}

Our results demonstrate that 5-HT and DA alter olfactory processing by modulating both distinct and shared features of odor-evoked responses. While both 5-HT and DA converge on many of the same AL neurons, the individual effects of each modulator diverge by targeting distinct phases of odor-evoked responses. We find that most projection neurons (PNs) integrate the influence of both 5-HT and DA, while a high proportion of putative local interneurons (LNs) were selectively modulated by 5-HT alone (although a small subset of LNs were affected by both modulators). Additionally, both modulators increased or decreased odor-evoked firing rate on a cell-by-cell basis, demonstrating that the individual effects of 5-HT and DA are variable within and across both functional cell types. Additionally, when both modulators were applied together, the effect of one modulator could override the other modulator. This suggests that while both 5- 
HT and DA can converge upon a single response feature, they likely target different $2^{\text {nd }}$ messengers causing a potential non-linear change to odor-evoked responses. Furthermore, the changes we observe may be due to direct and/or indirect modulation, to mediate the variable effects of both modulators that we observe. Altogether, our results suggest that DA modulates response timing of PNs by targeting intrinsic inhibitory phases of PNs (Figure 7A), while 5-HT alone may alter PN sensitivity at low odor concentrations by increasing PN response duration and altering the balance of lateral input via local interneurons (Figure 7B). Our results demonstrate that while 5-HT and DA simultaneously converge onto single AL neurons, they diverge by altering different odor-evoked response features (Figure 7C). Finally, past studies assessed the effects of modulators on populations of neurons by averaging the changes across the entire ensemble (Dacks et al., 2008; Dacks et al., 2012), effectively collapsing the individual changes to PNs and LNs together. While this is useful to assess population responses, this does not allow for in depth analysis of distinct response phases of each neuron. Here, we take a unique approach to assessing the effect of multiple modulators on the olfactory system by considering the effects of 5-HT and DA modulation on individual functional cell classes of the AL. Although the lack of a comprehensive atlas of 5-HT and DA receptor expression in the AL limits our ability gauge which effects are direct or indirect, our experimental design allows us to assess general principles of modulatory integration, an under-explored topic in sensory systems.

\section{5-HT and DA physiologically converge on most PNs while 5-HT selectively modulates the highest proportion of putative LNs}

Extrinsic neuromodulation is exerted by neurons that originate in independent networks and therefore provide information, such as physiological state, based on the activity of other neural networks (Lizbinski and Dacks, 2018). A number of extrinsic modulatory neurons project to the AL including two sets of widely projecting neurons releasing 5-HT (Kent et al., 1987; Dacks et al., 2006) and DA (Dacks et al., 2012). The contralaterally projecting serotoninimmunoreactive deutocerebral neuron (CSDn) (Kent et al., 1987; Dacks et al., 2006) and dopaminergic arching neurons (DAAR) (Dacks et al., 2012) project widely throughout the brain and innervate every glomerulus of the AL. However the CSDns and the DAARs project to different sub-regions within each glomerulus. The CSDns and DAARs neurons target partially overlapping glomerular regions to interact with LNs and PNs, yet the DAARs extends further 
into the region occupied by ORNs (Lizbinski et al., 2016). This suggested that both 5-HT and DA may converge onto common targets but also diverge by targeting distinct functional neuron types in the AL. Here, we find that most PNs integrate the influence of 5-HT and DA, while a higher proportion of putative LNs are selectively modulated by 5-HT. In vertebrates, DA mediates presynaptic inhibition of olfactory receptor neurons (ORNs) via activation of inhibitory D2, thereby reducing olfactory input to mitral and tufted cells (Koster et al., 1999; Hsia et al., 1999; Berkowicz and Trombley, 2000; Vaaga et al., 2017; Ennis et al., 2001). Based on DA's relative distribution with respect to ORNs in $M$. sexta glomeruli, DA may mediate presynaptic inhibition similar to vertebrates. Our results demonstrate that across all concentrations of odor, DA generally decreases odor-evoked responses of PNs (Figure 3E), suggesting the reduction in PN responses we observe may be partially mediated by reduced ORN input to PNs, implicating a potential role for DA in presynaptic inhibition. Previous studies report most AL neurons have their odor-evoked activity enhanced by DA (Dacks et al., 2012). However, this study assessed the effects of DA across the entire AL neuron population (both LNs and PNs). Therefore, this discrepancy may be due to the differences in comparing the effect of DA across the entire population vs. PNs only, and/or we recorded from different sub-populations of neurons that express different DA receptors.

We find the highest proportion of putative LNs are selectively modulated by 5-HT and many of our results demonstrate that 5-HT alters lateral interactions to alter AL sensitivity. A small subset of GABAergic LNs in M. sexta express the 5-HT1a receptor (Dacks et al., 2013), demonstrating that 5-HT can directly inhibit AL LNs. This is consistent with previous work showing that presumed LNs grown in cell culture are directly modulated by 5-HT (Mercer et al., 1996) and that 5-HT enhances a large proportion of AL neurons (Kloppenberg et al., 1999; Dacks et al., 2008). In M. sexta, serotonergic neurons do not extend far enough distally within glomeruli into the layer occupied by ORNs (Lizbinski et al., 2016). This suggests that the CSDns do not synaptically interact with ORNs and is consistent with work in the D. melanogaster AL showing that the CSDns do not synapse onto ORNs (Coates et al., 2017). However, previous studies have found that presynaptic inhibition of ORNs is enhanced by 5-HT despite 5-HT having no direct effect on ORNs (Dacks et al., 2009) and ORNS in D. melanogaster consistently express the 5-HT2B receptor (Sizemore and Dacks, 2016), suggesting that 5-HT modulates ORN activity either through lateral interactions or directly via non-synaptic sources. ORNs are 
continuously subjected to the influence of circulating neuromodulators in the hemolymph (Schendzielorz et al., 2015) and the mRNA for four of the M. sexta 5-HT receptors are expressed in the antennae, far from the axons of CSD neurons in the AL (Dacks et al., 2006; Dacks et al., 2013), although it is possible that 5-HT release from the CSDns may act at a distance to influence ORNs in an as yet unobserved manner. It should be noted that in D. melanogaster, exogenous application of 5-HT vs endogenous release have opposite effects, resulting in enhanced and decreased AL responses respectively, suggesting that synaptic vs. non-synaptic sources of 5-HT may differentially modulate AL activity (Zhang and Gaudry, 2016). Therefore, future work should determine the contribution of both the synaptic and non-synaptic sources of neuromodulation within the AL. Overall, while bath application does not allow us to assess the consequences of directly activating modulatory neurons within the AL, our experimental design does allow us to assess the general principles of modulatory integration, an under-unexplored phenomenon in sensory systems.

\section{The effects of individual modulators are variable across AL neurons}

A single modulator can exhibit variable effects on odor-evoked activity. For example, even within the same functional cell class, neurons could have their odor-evoked activity increased or decreased by a given modulator on a cell-by-cell basis. Here we find that the individual effects of 5-HT and DA on odor-evoked firing rate were highly variable within functional neuron type. In PNs, 5-HT both increased and decreased the firing rate of equal proportions of PNs, while DA decreased firing rate in a larger proportion of PNs. In LNs, both modulators increased and decreased pure excitation as well as rebound excitation. This suggests that within both classes, there are subsets of neurons that express different combinations of inhibitory and excitatory 5-HT and DA receptors, resulting in increased and/or decreased odorevoked firing rate. For example, in D. melanogaster, each AL neuronal class expresses a different combination of 5-HT receptors (Sizemore and Dacks, 2016). However, any given receptor is only expressed by a subset of neurons within that class (Sizemore and Dacks, 2016) which likely contributes to the non-uniform effects of 5-HT. A small subset of GABAergic LNs within the AL of M. sexta ( 24 out of $\sim 180$ total LNs) express the inhibitory Ms5HT1A receptor (Dacks et al., 2013). Furthermore, Ms5HT1B, Ms5HT2 and Ms5HT7 receptor mRNAs are expressed in the AL (Dacks et al., 2006b; Dacks et al., 2013) as are the mRNAs for the MsDop1, 
MsDop2 and MsINDR receptors (Dacks et al., 2012). However, cell-class specific receptor expression in $M$. sexta is still relatively unexplored.

The variable effects of neuromodulation may also be due to the expression of multiple modulatory receptors by a single neuron within a neuron class. G-protein coupled receptors can target different second-messengers or target the same second messenger, but in opposing manners. For example, the 5-HT7 and 5-HT2 receptors affect cAMP and IP3 levels respectively, while both the 5-HT7 and 5-HT1a receptors affect cAMP levels, but in opposing manners. Therefore a signal modulator can molecularly converge and/or diverge based on the receptor types they affect. We find that when both modulators were applied together, many of the individual effects of each modulator, such as enhancement of firing rate or changes to inhibition were attenuated and/or non-linear. For example, the percentage of total PNs that had their odorevoked firing altered by the simultaneous application of 5-HT and DA was not proportionally consistent with the number of PNs affected individually by either modulator. This suggests that while 5-HT and DA may individually increase or decrease firing rate in the same neuron, their integrated effects are non-linear and even subtractive. This is likely due to differential receptor expression, where subsets of AL neurons express receptors which are coupled to opposing second-messenger cascades. Receptors for 5-HT and DA have the capacity to converge upon the same biochemical cascades as many couple to adenylyl cyclase to affect cAMP levels (Saudou et al., 1992; Colas et al., 1995; Mustard et al., 2005). However, the biochemical consequences of activating 5-HT and DA receptors also have the opportunity to diverge, as the 5-HT1 and 5-HT2 receptor subtypes affect $\mathrm{IP}_{3}$ pathways while DA receptors and the 5-HT7 receptor do not (Saudou et al., 1992; Mustard et al., 2005). Since there are currently no reliable antibodies to label for most M. sexta 5-HT and DA receptors, future studies should address the cell-class specific receptor expression using in situ hybridization. The non-linear effects could also be due

to altered synaptic input to the neurons being recorded. Furthermore, biophysical properties and response tuning of individual AL neurons further complicate the effects of each modulator.

\section{5-HT and DA modulate shared and distinct odor-evoked phases of projection neurons}

Within a single neuron, multiple modulators can affect the same response features or target distinct features. Here, we demonstrate that 5-HT and DA differentially modulate olfactory coding by targeting distinct and shared response features of PNs. DA likely modulates response 
timing by decreasing the length of intrinsic inhibitory phases of PNs while 5-HT likely mediates changes in PN sensitivity by altering lateral input to PNs via LNs, thereby altering the strength and duration of odor-evoked responses. DA generally decreased the GABAa sensitive I1 phase of PNs. The amount of inhibitory input a cell receives alters the amplitude of I1 (Heinbockel et al., 1999). GABAa and mediated inhibition plays an important role in the spatiotemporal responses of AL neurons by increasing the difference between odor-representations and allowing AL to track odor-pulses in rapid succession (Wilson and Laurent 2005; Tripathy et al., 2010; Lei et al., 2009). The importance of a spatiotemporal code in differentiating between molecular similar and distinct odors has been demonstrated (Daly et al., 2004; Daly et al., 2016), and likely reflects alterations in lateral interactions between and across glomeruli to enhance contrast. The I2 phase of PNs affects the fidelity with which PNs can encode the temporal dynamics of odor stimuli by hyperpolarizing them for long periods of time, thus preventing rapid succession of excitatory PN responses to odors (Christensen et al., 1998; Heinbockel et al., 1999; Lei et al., 2009). Previous studies have shown that DA reduced the post-excitation period of spike suppression in PNs called the I2 phase (Dacks et al., 2012) that typically lasts between 10ms and 1.5 seconds (Christensen et al., 1998). Our results are consistent with this finding, as we found that DA alone decreases the length of I2. Overall, our results suggest that DA modulates the response timing of PNs by altering the inhibitory phases of PN responses.

At low odor-concentrations, PN response duration and response integral was increased by 5-HT alone. This effect is likely mediated in part by decreased indirect inhibition from LNs onto PNs, allowing for a longer, stronger response. The 5-HT mediated increase in response duration/integral and general increase in firing rate across all odor concentrations suggests that 5HT likely mediates changes in sensitivity, increasing the duration of PN responses at low odor concentrations. The effects of 5-HT on PNs were also largely concentration specific, however only a small proportion of PNs had their odor-evoked firing rate selectively modulated by 5-HT. This suggests the increase in PN response duration is mediated by changes to the synaptic input (via LNs) to that PN.

\section{5-HT and DA converge on the same feature of odor-evoked responses in local interneurons} but may modulate it via distinct mechanisms 
We found that while both 5-HT and DA can affect the same features of odor-evoked responses, they may do so via distinct mechanisms. For example, rebound excitation in LNs was modulated by both 5-HT and DA (multiple modulators converging onto a single response feature), but 5-HT alone decreased odor-evoked inhibition in LNs. Rebound excitation is common across species and neuron type (Homberg 1984; Mulloney \& Selverston 1974, Satterlie 1985; Bertrand and Cazalets, 1998; Johnson \& Getting 1987) and is mediated by ion channels that become active at a membrane potential below rest. If a neuron is hyperpolarized, these channels open and when inhibition is relieved, the neuron will depolarize past rest, potentially increasing the firing rate (Getting 1989). The strength of rebound excitation can be altered by 1) modulating $\mathrm{I}_{\mathrm{h}}$ or $\mathrm{I}_{\mathrm{CaT}}$ channels directly and/or 2) altering the amount of inhibition exerted upon the $\mathrm{LN}$, and therefore the probability that $\mathrm{I}_{\mathrm{h}}$ or $\mathrm{I}_{\mathrm{CaT}}$ channels will open. 5-HT decreased the duration of odor-evoked inhibition in LNs potentially increasing rebound excitation by altering the length of inhibitory drive a given LN has onto other LNs. DA on the other hand did not affect the duration of odor-evoked inhibition and therefore likely affected post-inhibitory rebound by directly altering the channels intrinsic to LNs. In vivo studies of cultured honeybee AL neurons demonstrate that DA modulates two $\mathrm{Ca}^{2+}$ dependent $\mathrm{K}^{+}$currents $\left(\mathrm{I}_{\mathrm{KC}}\right)$; DA reduces a slowly activating outward conductance ( $\left.\mathrm{I}_{\mathrm{KCsustained}}\right)$ and, reduces a rapidly activating outward conductance (IKCtransient), thereby altering the excitability of AL neurons (Perk et al., 2006). In the stomatogastric ganglion of crustaceans, DA modulates a voltage sensitive transient $\mathrm{K}^{+}$current, resulting in increased rebound excitation in the presence of DA (Harris-Warrick et al., 1995; Kloppenburg et al., 1999). While the effects of DA on $\mathrm{K}^{+}$currents are unknown in M. sexta, modulation of intrinsic conductances could explain the DA mediated increase in post-inhibitory rebound.

Physiologically, bath application of 5-HT reduces two $\mathrm{K}^{+}$conductances, a sustained $\mathrm{I}_{\mathrm{KV}^{-}}$ like conductance and a more transient $\mathrm{I}_{\mathrm{A}}$-like conductance, and lowers the voltage of inactivation for the $\mathrm{I}_{\mathrm{A}}$-like conductance in PNs (Kloppenburg et al., 1999) and cultured LNs (Mercer et al., 1995). As a consequence, the resistance (and therefore the excitability) of LNs and PNs is increased by 5-HT (Kloppenburg and Hildebrand, 1995) and the odor-evoked responses of some AL neurons is also increased (Kloppenburg et al., 1999; Dacks et al., 2008). We found that the large majority of LNs had their odor-evoked activity modulated by 5-HT, with a strong effect on increased odor-evoked firing at low odor-concentrations. This suggests that complex LN-LN 
interactions may be modulated by 5-HT to alter the mix of excitation/inhibition onto PNs. At low odor-concentrations, PN response duration and response integral was increased by 5-HT alone. This effect is likely mediated in part by decreased indirect inhibition from LNs onto PNs, allowing for a longer, stronger response. Other studies have demonstrated that 5-HT increases the slope of odor-evoked responses as a function of odor concentration (Dacks et al., 2008), suggesting that 5-HT modulates the gain of the AL. We did not detect changes in gain by either modulator which is likely a result of how our responses were quantified. Past studies assessed the effects of modulators on populations of neurons by averaging the modulator's effect across the entire ensemble, effectively collapsing changes to PNs and LNs together. Our approach assesses the effects of each modulator on each functional cell type, LNs and PNs, separately. Thus, while 5-HT may increase the gain of AL neurons across the entire population, we were unable to detect a significant change in slope across odor-concentrations. Although we did not detect changes in gain, our results along with previous work on 5-HT modulation in the AL (Dacks et al., 2008) suggest that 5-HT modulates the sensitivity of AL neurons. Our results along with the findings from Dacks et al., 2008 suggest that 5-HT modulates the sensitivity of AL neurons by 1) selectively altering the length of odor-evoked inhibition of LNs and 2) increasing the response duration of PNs, likely due to relieved inhibitory drive onto PNs.

\section{Physiological context of multiple modulator release}

5-HT and DA are likely released under different behavioral contexts (Kloppenburg and Hildebrand, 1995; Dacks et al., 2008; Kloppenburg and Mercer, 2008; Dacks et al., 2012). For example, the levels of 5-HT in the ALs of M. sexta fluctuate throughout the day peaking at dawn and dusk (Kloppenburg et al., 1999), when M. sexta perform the majority of their odor-guided behaviors (Lingren et al., 1977). Furthermore, injection of 5-HT into cabbage looper moths broadens the range of the day in which male moths will fly to female pheromone (Linn and Roelofs, 1986). This suggests that the release of 5-HT by serotonergic extrinsic neurons is at least, in part, regulated by the waking state. Within the AL of M. sexta, DA is associated with the context of aversive stimuli, as DA receptor antagonists injected into the AL block aversive (Dacks et al., 2012), but not appetitive conditioning (Riffell et al., 2013). In mosquitos, manipulating D1 receptors using D1 receptor antagonists, D1 receptor specific RNAi, and whole body CRISPR-Cas9 against D1, suppressed olfactory learning (Vinauger et al., 2018). 
Specifically, for RNAi experiments, D1 receptor manipulation affected mosquitoes' ability to learn aversive information but did not affect innate olfactory behavior. This evidence, along with behavioral basis of DA and aversion in M. sexta (Dacks et al., 2012), demonstrates that DA likely operates in the context of aversion. Both the $\mathrm{AL}$ and the mushroom bodies, the center for learning and memory in insects, likely mediate this aversion, as dopamine plays a central role in olfactory learning (Unoki et al., 2005; Schwaerzel et al 2003; Kim et al, 2007; Schroll et al., 2006).

The complexity of modulatory integration we observe here is likely conserved within the olfactory system of many species as the potential for convergent modulatory influence is present in the olfactory system of both invertebrate and vertebrates (Lizbinski and Dacks, 2018). Most, if not all insect glomeruli receive input from multiple extrinsic sources that release 5-HT (Kent et al., 1987; Rehder et al., 1987; Salecker and Distler, 1990; Sun et al., 1993; Wegerhoff, 1999; Hill et al., 2002; Dacks et al., 2006a; Roy et al., 2007), DA (Dacks et al, 2012), and octopamine (Dacks et al., 2005; Sinakevitch et al., 2005; Sinakevitch and Strausfeld, 2006). Furthermore, AL LNs release a heterogeneous mix of GABA and neuropeptides (Carlsson et al., 2010; Kromann et al., 2013; Siju et al., 2013; Lizbinski et al 2018), that modulate olfactory processing within the context of previous and ongoing network processing. The mechanisms by which single olfactory neurons integrate multiple modulators are highly complex, with subsets of $\mathrm{AL}$ neurons employing multiple strategies of convergence and divergence of single modulators. This is further complicated when multiple modulators converge onto a single neuron. Our work demonstrates that, while both 5-HT and DA converge on many of the same AL neurons, the individual effects of each modulator diverge by targeting distinct phases of odor-evoked responses to mediate network-wide changes in olfactory processing under different physiological contexts.

\section{Acknowledgements:}

We thank Gary Marsat for his help with writing MatLab scripts for analysis and for Kevin Daly's lab for raising the moths.

\section{Works cited:}


Beliez, L., Barrière, G., Bertrand, S. S., \& Cazalets, J. R. (2014). Multiple monoaminergic modulation of posturo-locomotor network activity in the newborn rat spinal cord. Frontiers in neural circuits, 8, 99.

Bell, R. A., \& Joachim, F. G. (1976). Techniques for rearing laboratory colonies of tobacco hornworms and pink bollworms. Annals of the Entomological Society of America, 69(2), 365-373.

Berkowicz, D. A., \& Trombley, P. Q. (2000). Dopaminergic modulation at the olfactory nerve synapse. Brain research, 855(1), 90-99.

Bertrand, S., \& Cazalets, J. R. (1998). Postinhibitory rebound during locomotor-like activity in neonatal rat motoneurons in vitro. Journal of neurophysiology, 79(1), 342-351.

Blitz, D. M., \& Nusbaum, M. P. (1999). Distinct functions for cotransmitters mediating motor pattern selection. Journal of Neuroscience, 19(16), 6774-6783.

Brezina, V., Orekhova, I. V., \& Weiss, K. R. (1996). Functional uncoupling of linked neurotransmitter effects by combinatorial convergence. Science, 273(5276), 806-810.

Carlsson, M. A., Diesner, M., Schachtner, J., \& Nässel, D. R. (2010). Multiple neuropeptides in the Drosophila antennal lobe suggest complex modulatory circuits. Journal of Comparative Neurology, 518(16), 3359-3380.

Chou, Y. H., Spletter, M. L., Yaksi, E., Leong, J. C., Wilson, R. I., \& Luo, L. (2010). Diversity and wiring variability of olfactory local interneurons in the Drosophila antennal lobe. Nature neuroscience, 13(4), 439.

Christensen, T. A., \& Hildebrand, J. G. (1987). Male-specific, sex pheromone-selective projection neurons in the antennal lobes of the mothManduca sexta. Journal of comparative Physiology A, 160(5), 553-569.

Christensen, T. A., Waldrop, B. R., Harrow, I. D., \& Hildebrand, J. G. (1993). Local interneurons and information processing in the olfactory glomeruli of the moth Manduca sexta. Journal of Comparative Physiology A, 173(4), 385-399.

Christensen, T. A., Waldrop, B. R., \& Hildebrand, J. G. (1998). Multitasking in the olfactory system: context-dependent responses to odors reveal dual GABA-regulated coding mechanisms in single olfactory projection neurons. Journal of Neuroscience, 18(15), 5999-6008.

Coates, K. E., Majot, A. T., Zhang, X., Michael, C. T., Spitzer, S. L., Gaudry, Q., \& Dacks, A. M. (2017). Identified serotonergic modulatory neurons have heterogeneous synaptic connectivity within the olfactory system of Drosophila. Journal of Neuroscience, 0192-17.

Colas, J. F., Launay, J. M., Kellermann, O., Rosay, P., \& Maroteaux, L. (1995). Drosophila 5-HT2 serotonin receptor: coexpression with fushi-tarazu during segmentation. Proceedings of the National Academy of Sciences, 92(12), 5441-5445.

Dacks, A. M., Christensen, T. A., Agricola, H. J., Wollweber, L., \& Hildebrand, J. G. (2005).

Octopamine- immunoreactive neurons in the brain and subesophageal ganglion of the hawkmoth Manduca $\quad$ sexta. Journal of Comparative Neurology, 488(3), 255-268.

Dacks, A. M., Christensen, T. A., \& Hildebrand, J. G. (2006). Phylogeny of a serotonin-immunoreactive neuron in the primary olfactory center of the insect brain. Journal of Comparative Neurology, 498(6), 727-746. 
Dacks, A. M., Christensen, T. A., \& Hildebrand, J. G. (2008). Modulation of olfactory information processing in the antennal lobe of Manduca sexta by serotonin. Journal of neurophysiology, 99(5), 2077-2085.

Dacks, A. M., Green, D. S., Root, C. M., Nighorn, A. J., \& Wang, J. W. (2009). Serotonin modulates olfactory processing in the antennal lobe of Drosophila. Journal of neurogenetics, 23(4), 366377.

Dacks, A. M., Riffell, J. A., Martin, J. P., Gage, S. L., \& Nighorn, A. J. (2012). Olfactory modulation by dopamine in the context of aversive learning. Journal of neurophysiology, 108(2), 539-550.

Dacks, A. M., Reale, V., Pi, Y., Zhang, W., Dacks, J. B., Nighorn, A. J., \& Evans, P. D. (2013). A characterization of the Manduca sexta serotonin receptors in the context of olfactory neuromodulation. PloS one, 8(7), e69422.

Daly, K. C., Wright, G. A., \& Smith, B. H. (2004). Molecular features of odorants systematically

influence slow temporal responses across clusters of coordinated antennal lobe units in the moth Manduca sexta. Journal of neurophysiology, 92(1), 236-254.

Daly, K. C., Kalwar, F., Hatfield, M., Staudacher, E., \& Bradley, S. P. (2013). Odor detection in Manduca sexta is optimized when odor stimuli are pulsed at a frequency matching the wing beat during flight. PLoS One, 8(11), e81863.

Daly, K. C., Bradley, S., Chapman, P. D., Staudacher, E. M., Tiede, R., \& Schachtner, J. (2016). Space takes time: Concentration dependent output codes from primary olfactory networks rapidly provide additional information at defined discrimination thresholds. Frontiers in cellular neuroscience, 9 , 515 .

Ennis, M., Zhou, F. M., Ciombor, K. J., Aroniadou-Anderjaska, V., Hayar, A., Borrelli, E., ... \& Shipley,

M. T. (2001). Dopamine D2 receptor-mediated presynaptic inhibition of olfactory nerve terminals. Journal of Neurophysiology, 86(6), 2986-2997.

Getting, P. A. (1989). Emerging principles governing the operation of neural networks. Annual review of neuroscience, 12(1), 185-204.

Gutierrez, G. J., \& Marder, E. (2014). Modulation of a single neuron has state-dependent actions on circuit dynamics. Eneuro, 1(1), ENEURO-0009

Harris-Warrick, R. M., Coniglio, L. M., Barazangi, N., Guckenheimer, J., \& Gueron, S. (1995).

Dopamine modulation of transient potassium current evokes phase shifts in a central pattern

generator network. Journal of Neuroscience, 15(1), 342-358.

Heinbockel, T., Christensen, T. A., \& Hildebrand, J. G. (1999). Temporal tuning of odor responses in pheromone-responsive projection neurons in the brain of the sphinx moth Manduca sexta. Journal of Comparative Neurology, 409(1), 1-12.

Hill, E. S., Iwano, M., Gatellier, L., \& Kanzaki, R. (2002). Morphology and physiology of the serotoninimmunoreactive putative antennal lobe feedback neuron in the male silkmoth Bombyx mori. Chemical senses, 27(5), 475-483.

Homberg, U. (1984). Processing of antennal information in extrinsic mushroom body neurons of the bee brain. Journal of Comparative Physiology A, 154(6), 825-836.

Hsia, A. Y., Vincent, J. D., \& Lledo, P. M. (1999). Dopamine depresses synaptic inputs into the olfactory bulb. Journal of neurophysiology, 82(2), 1082-1085. 
Katz, P. S., \& Frost, W. N. (1995). Intrinsic neuromodulation in the Tritonia swim CPG: serotonin mediates both neuromodulation and neurotransmission by the dorsal swim interneurons. Journal of Neurophysiology, 74(6), 2281-2294.

Kent, K. S., Hoskins, S. G., \& Hildebrand, J. G. (1987). A novel serotonin-immunoreactive neuron in the antennal lobe of the sphinx moth Manduca sexta persists throughout postembryonic life. Journal of neurobiology, 18(5), 451-465.

Khawaled, R., Bruening-Wright, A., Adelman, J. P., \& Maylie, J. (1999). Bicuculline block of smallconductance calcium-activated potassium channels. Pflügers Archiv, 438(3), 314-321.

Kim, Y. C., Lee, H. G., \& Han, K. A. (2007). D1 dopamine receptor dDA1 is required in the mushroom body neurons for aversive and appetitive learning in Drosophila. Journal of Neuroscience, 27(29), $7640-7647$.

Kloppenburg, P., \& Hildebrand, J. G. (1995). Neuromodulation by 5-hydroxytryptamine in the antennal lobe of the sphinx moth Manduca sexta. Journal of Experimental Biology, 198(3), 603-611.

Kloppenburg, P., Levini, R. M., \& Harris-Warrick, R. M. (1999). Dopamine modulates two potassium currents and inhibits the intrinsic firing properties of an identified motor neuron in a central pattern generator network. Journal of Neurophysiology, 81(1), 29-38.

Kloppenburg, P., Ferns, D., \& Mercer, A. R. (1999). Serotonin enhances central olfactory neuron responses to female sex pheromone in the male sphinx moth Manduca sexta. Journal of Neuroscience, 19(19), 8172-8181.

Koster, N. L., Norman, A. B., Richtand, N. M., Nickell, W. T., Puche, A. C., Pixley, S. K., \& Shipley, M.

T. (1999). Olfactory receptor neurons express D2 dopamine receptors. Journal of Comparative Neurology, 411(4), 666-673.

Kromann, S. H., Hansson, B. S., \& Ignell, R. (2013). Distribution of neuropeptides in the antennal lobes

of male Spodoptera littoralis. Cell and tissue research, 354(2), 431-440.

Lei, H., Christensen, T. A., \& Hildebrand, J. G. (2002). Local inhibition modulates odor-evoked synchronization of glomerulus-specific output neurons. Nature neuroscience, 5(6), 557.

Lei, H., Riffell, J. A., Gage, S. L., \& Hildebrand, J. G. (2009). Contrast enhancement of stimulus intermittency in a primary olfactory network and its behavioral significance. Journal of Biology, 8(2), 21.

Li, X., Bucher, D., \& Nadim, F. (2018). Distinct co-modulation rules of synapses and voltage-gated currents coordinate interactions of multiple neuromodulators. Journal of Neuroscience, 38(40), 8549-8562.

Lingren, P. D., Greene, G. L., Davis, D. R., Baumhover, A. H., \& Henneberry, T. J. (1977). Nocturnal behavior of four lepidopteran pests that attack tobacco and other crops. Annals of the Entomological Society of America, 70(2), 161-167.

Lizbinski, K. M., Metheny, J. D., Bradley, S. P., Kesari, A., \& Dacks, A. M. (2016). The anatomical basis

for modulatory convergence in the antennal lobe of Manduca sexta. Journal of Comparative Neurology, 524(9), 1859-1875.

Lizbinski, K. M., \& Dacks, A. M. (2018). Intrinsic and extrinsic neuromodulation of olfactory processing. Frontiers in cellular neuroscience, 11, 424. 
Lizbinski, K. M., Marsat, G., \& Dacks, A. M. (2018). Systematic Analysis of Transmitter Coexpression Reveals Organizing Principles of Local Interneuron Heterogeneity. eNeuro, 5(5).

Marder, E., \& Thirumalai, V. (2002). Cellular, synaptic and network effects of neuromodulation. Neural Networks, 15(4), 479-493.

Marder, E. (2012). Neuromodulation of neuronal circuits: back to the future. Neuron, 76(1), 1-11.

Marder, E., O'Leary, T., \& Shruti, S. (2014). Neuromodulation of circuits with variable parameters: single neurons and small circuits reveal principles of state-dependent and robust neuromodulation. Annual Review of Neuroscience, 37, 329-346

Martin, J. P., Beyerlein, A., Dacks, A. M., Reisenman, C. E., Riffell, J. A., Lei, H., \& Hildebrand, J. G. (2011). The neurobiology of insect olfaction: sensory processing in a comparative context. Progress in neurobiology, 95(3), 427-447.

Mesce, K. A., Crisp, K. M., \& Gilchrist, L. S. (2001). Mixtures of octopamine and serotonin have nonadditive effects on the CNS of the medicinal leech. Journal of neurophysiology, 85(5), 20392046.

Mercer, A. R., Kloppenburg, P., \& Hildebrand, J. G. (1996). Serotonin-induced changes in the excitability of cultured antennal-lobe neurons of the sphinx moth Manduca sexta. Journal of Comparative Physiology A, 178(1), 21-31.

Mustard, J. A., Beggs, K. T., \& Mercer, A. R. (2005). Molecular biology of the invertebrate dopamine receptors. Archives of Insect Biochemistry and Physiology: Published in Collaboration with the Entomological Society of America, 59(3), 103-117.

Nadim, F., \& Bucher, D. (2014). Neuromodulation of neurons and synapses. Current opinion in neurobiology, 29, 48-56.

Olsen, S. R., \& Wilson, R. I. (2008). Lateral presynaptic inhibition mediates gain control in an olfactory circuit. Nature, 452(7190), 956.

Perk, C. G., \& Mercer, A. R. (2006). Dopamine modulation of honey bee (Apis mellifera) antennal-lobe neurons. Journal of neurophysiology, 95(2), 1147-1157.

Perkel, D. H., \& Mulloney, B. (1974). Motor pattern production in reciprocally inhibitory neurons exhibiting postinhibitory rebound. Science, 185(4146), 181-183.

Rehder, V., Bicker, G., \& Hammer, M. (1987). Serotonin-immunoreactive neurons in the antennal lobes and suboesophageal ganglion of the honeybee. Cell and tissue research, 247(1), 59-66.

Reisenman, C. E., Dacks, A. M., \& Hildebrand, J. G. (2011). Local interneuron diversity in the primary olfactory center of the moth Manduca sexta. Journal of comparative Physiology A, 197(6), 653665.

Riffell, J. A., Alarcón, R., Abrell, L., Davidowitz, G., Bronstein, J. L., \& Hildebrand, J. G. (2008).

Behavioral consequences of innate preferences and olfactory learning in hawkmoth-flower interactions. Proceedings of the National Academy of Sciences, 105(9), 3404-3409.

Riffell, J. A., Lei, H., \& Hildebrand, J. G. (2009). Neural correlates of behavior in the moth Manduca sexta in response to complex odors. Proceedings of the National Academy of Sciences, pnas0910592106.

Riffell, J. A., Lei, H., Abrell, L., \& Hildebrand, J. G. (2013). Neural basis of a pollinator's buffet: olfactory specialization and learning in Manduca sexta. Science, 339(6116), 200-204. 
Root, C. M., Masuyama, K., Green, D. S., Enell, L. E., Nässel, D. R., Lee, C. H., \& Wang, J. W. (2008).

A presynaptic gain control mechanism fine-tunes olfactory behavior. Neuron, 59(2), 311-321.

Roy, B., Singh, A. P., Shetty, C., Chaudhary, V., North, A., Landgraf, M., ... \& Rodrigues, V. (2007). Metamorphosis of an identified serotonergic neuron in the Drosophila olfactory system. Neural development, 2(1), 20.

Salecker, I., \& Distler, P. (1990). Serotonin-immunoreactive neurons in the antennal lobes of the American cockroach Periplaneta americana: light-and electron-microscopic observations. Histochemistry, 94(5), 463-473.

Satterlie, R. A. (1985). Reciprocal inhibition and postinhibitory rebound produce reverberation in a locomotor pattern generator. Science, 229(4711), 402-404.

Saudou, F., Boschert, U., Amlaiky, N., Plassat, J. L., \& Hen, R. (1992). A family of Drosophila serotonin receptors with distinct intracellular signalling properties and expression patterns. The EMBO journal, 11(1), 7-17.

Schendzielorz, T., Schirmer, K., Stolte, P., \& Stengl, M. (2015). Octopamine regulates antennal sensory neurons via daytime-dependent changes in cAMP and IP3 levels in the hawkmoth Manduca sexta. PLoS One, 10(3), e0121230.

Schroll, C., Riemensperger, T., Bucher, D., Ehmer, J., Völler, T., Erbguth, K., ... \& Fiala, A. (2006).

Light- induced activation of distinct modulatory neurons triggers appetitive or aversive learning in Drosophila larvae. Current biology, 16(17), 1741-1747.

Schwaerzel, M., Monastirioti, M., Scholz, H., Friggi-Grelin, F., Birman, S., \& Heisenberg, M. (2003). Dopamine and octopamine differentiate between aversive and appetitive olfactory memories in Drosophila. Journal of Neuroscience, 23(33), 10495-10502.

Seki, Y., Rybak, J., Wicher, D., Sachse, S., \& Hansson, B. S. (2010). Physiological and morphological characterization of local interneurons in the Drosophila antennal lobe. Journal of neurophysiology, 104(2), 1007-1019.

Siju, K. P., Reifenrath, A., Scheiblich, H., Neupert, S., Predel, R., Hansson, B. S., ... \& Ignell, R. (2014). Neuropeptides in the antennal lobe of the yellow fever mosquito, Aedes aegypti. Journal of Comparative Neurology, 522(3), 592-608.

Sinakevitch, I., Niwa, M., \& Strausfeld, N. J. (2005). Octopamine-like immunoreactivity in the honey bee and cockroach: Comparable organization in the brain and subesophageal ganglion. Journal of Comparative Neurology, 488(3), 233-254.

Sinakevitch, I., \& Strausfeld, N. J. (2006). Comparison of octopamine-like immunoreactivity in the brains of the fruit fly and blow fly. Journal of Comparative Neurology, 494(3), 460-475.

Sizemore, T. R., \& Dacks, A. M. (2016). Serotonergic modulation differentially targets distinct network elements within the antennal lobe of Drosophila melanogaster. Scientific reports, 6, 37119.

Sun, X. J., Tolbert, L. P., \& Hildebrand, J. G. (1993). Ramification pattern and ultrastructural characteristics of the serotonin-immunoreactive neuron in the antennal lobe of the moth Manduca sexta: a laser scanning confocal and electron microscopic study. Journal of Comparative Neurology, 338(1), 5-16.

Swensen, A. M., \& Marder, E. (2000). Multiple peptides converge to activate the same voltagedependent current in a central pattern-generating circuit. Journal of Neuroscience, 20(18), 67526759 . 
Thirumalai, V., \& Marder, E. (2002). Colocalized neuropeptides activate a central pattern generator by acting on different circuit targets. Journal of Neuroscience, 22(5), 1874-1882.

Tripathy, S., Staudacher, E. M., Peters, O., Kalwar, F., Hatfield, M., \& Daly, K. (2010). Odors pulsed at wing beat frequencies are tracked by primary olfactory networks and enhance odor detection. Frontiers in cellular neuroscience, $4,1$.

Unoki, S., Matsumoto, Y., \& Mizunami, M. (2005). Participation of octopaminergic reward system and dopaminergic punishment system in insect olfactory learning revealed by pharmacological study. European journal of Neuroscience, 22(6), 1409-1416.

Vaaga, C. E., Yorgason, J. T., Williams, J. T., \& Westbrook, G. L. (2016). Presynaptic gain control by endogenous cotransmission of dopamine and GABA in the olfactory bulb. Journal of neurophysiology, 117(3), 1163-1170.

Vinauger, C., Lahondère, C., Wolff, G. H., Locke, L. T., Liaw, J. E., Parrish, J. Z., ... \& Riffell, J. A. (2018). Modulation of host learning in Aedes aegypti mosquitoes. Current Biology, 28(3), 333-344.

Waldrop, B., Christensen, T. A., \& Hildebrand, J. G. (1987). GABA-mediated synaptic inhibition of projection neurons in the antennal lobes of the sphinx moth, Manduca sexta. Journal of Comparative Physiology A: Neuroethology, Sensory, Neural, and Behavioral Physiology, 161(1), 23-32.

Wang, J. W. (2012). Presynaptic modulation of early olfactory processing in Drosophila. Developmental neurobiology, 72(1), 87-99.

Wegerhoff, R. (1999). GABA and serotonin immunoreactivity during postembryonic brain development in the beetle Tenebrio molitor. Microscopy research and technique, 45(3), 154-164.

Wilson, R. I., \& Laurent, G. (2005). Role of GABAergic inhibition in shaping odor-evoked spatiotemporal patterns in the Drosophila antennal lobe. Journal of Neuroscience, 25(40), 9069-9079.

Zhang, X., \& Gaudry, Q. (2016). Functional integration of a serotonergic neuron in the Drosophila antennal lobe. Elife, 5, e16836. 
Figures:

A.

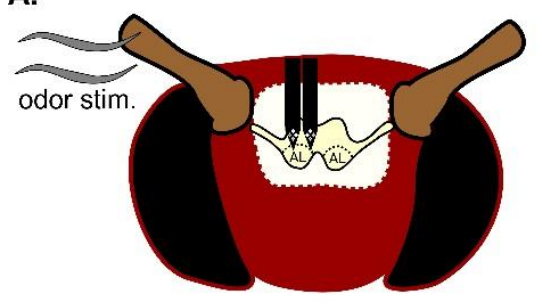

C.

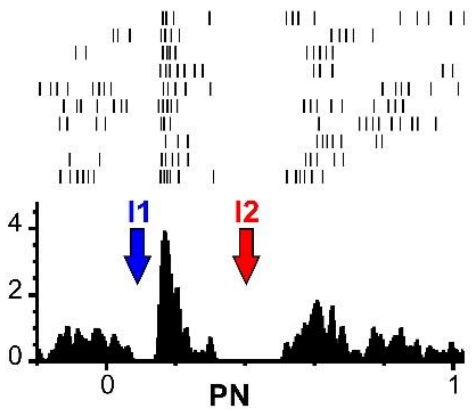

B.

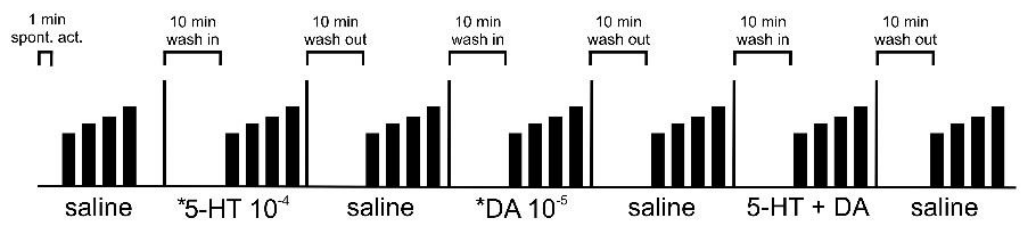

D.
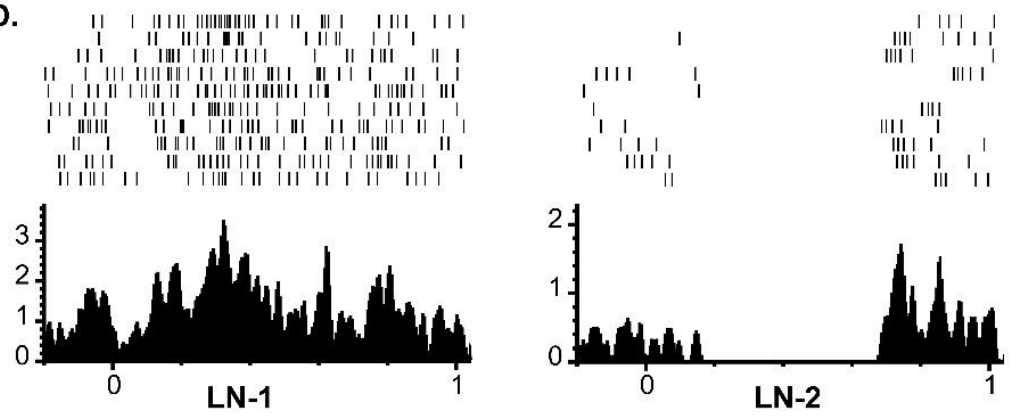

Figure 1: Experimental design and odor-evoked activity of projection neurons and local interneurons.

A: Schematic of extracellular tetrode electrophysiology. Moths were fixed with wax into copper tubes, a window cut into their head capsule to expose the antennal lobes (ALs) and M. sexta physiological saline was superfused over the brain. Probes were inserted into the AL allowing the recording of multiple AL neurons simultaneously. B: Electrophysiology experimental design. One minute of spontaneous activity was recorded in control saline before odor stimuli of increasing concentration of Datura wrightii floral blend odorant pulsed for $200 \mathrm{~ms}$ (depicted as black bars respectively increasing in size: $\left.10^{-4}, 10^{-3}, 10^{-2}, 10^{-1}\right), 10$ times with an inter pulse interval of 10 seconds. Modulators were bath applied for a 10 minute wash in period before odors were pulsed. One minute of spontaneous activity was recorded before odor presentation during each saline and modulator wash-in period. The order of 5-HT and DA was randomized across experiments. The combined 5-HT +DA wash was always presented last. Modulators were washed out for 10 minutes by saline between each application and odors were presented as described above. C: Odor-evoked activity of a projection neuron (PN). PNs exhibit stereotyped odor-evoked activity that contain 3 phases, a brief pre-excitation inhibitory phase called, $\mid 1$. This is followed by the excitation phase (E), and then an epoch of inhibition of variable length called 12 which is typically lasts between $10 \mathrm{~ms}$ to $1.5 \mathrm{~s}$. D: Odor-evoked activity of putative LNs falls within two categories: excited by odors (LN-1), or inhibited by odors (LN-2). The distinct odor-evoked activity of PNs and putative LNs allows us to group units into their respective functional classes. 
A.

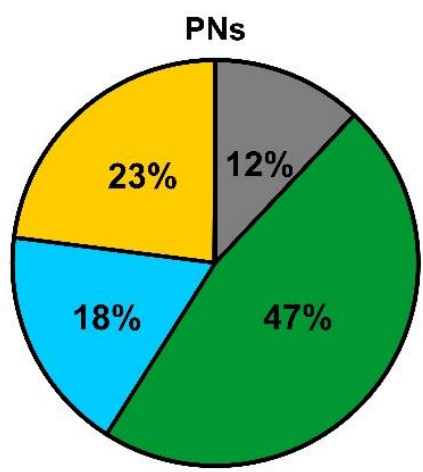

5HT DA 5HT+DA unaffected
B.

odor evoked firing rate PNs

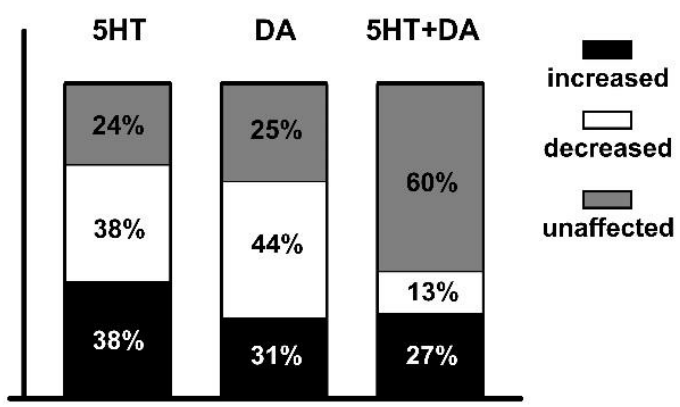

c.

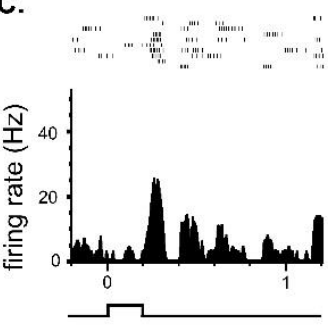

E.

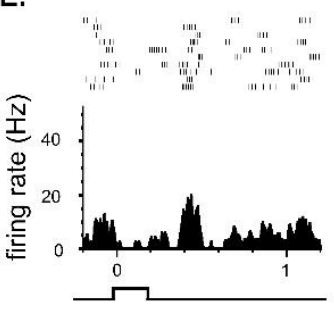

G.

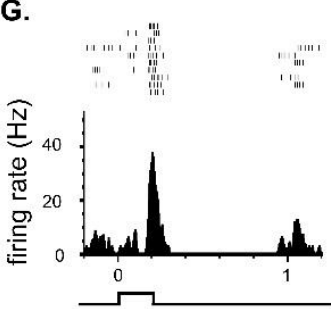

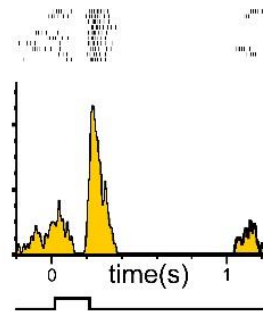
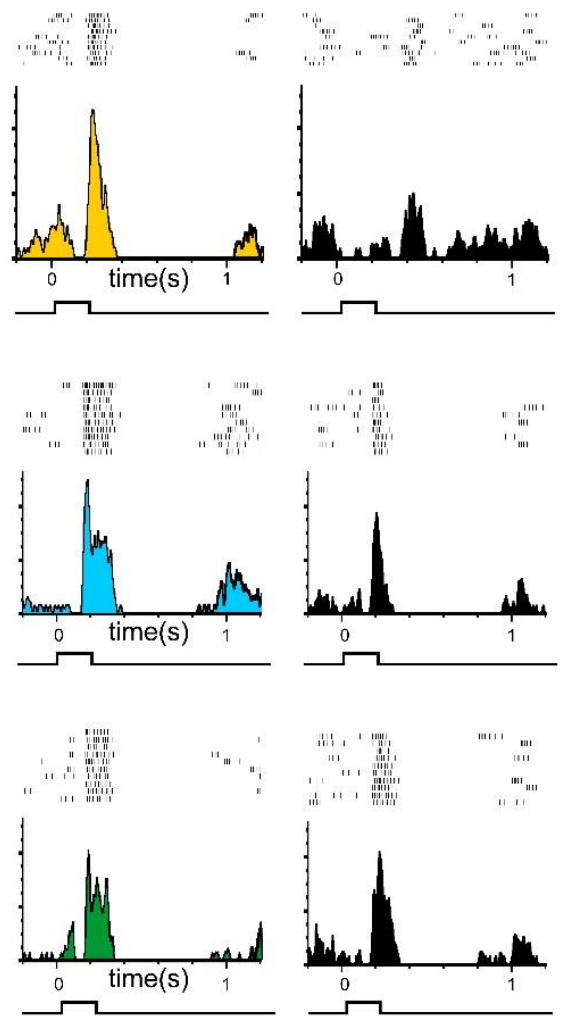

D.

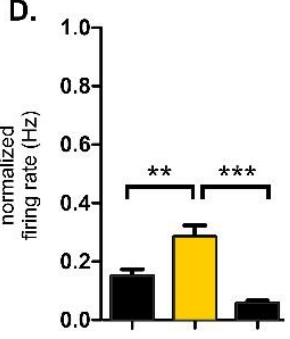

F.
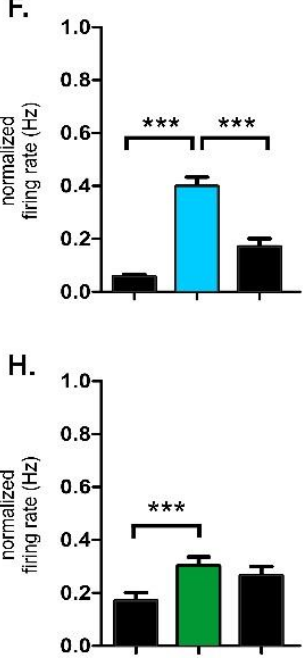

Figure 2: Serotonin and Dopamine modulate odor-evoked firing rate in projection neurons and their integrated effects are non-linear.

A: Within the total population of PNs recorded, sub-populations showed varied responses to each modulator. The largest proportion of PNs were affected by both modulator. Smaller sub-sets were selectively modulated by only $5-\mathrm{HT}$ and only DA. There was also a small sub-set that was unaffected by either modulator. B: 5-HT and DA have variable effects on population of PNs. Both 5-HT and DA increased and decreased odor-evoked firing rate of PNs. In some neurons, both modulators had opposing effects on odor-evoked firing rate on the same PN. The integrated effects of 5-HT and DA were generally non-linear. For example, even in PNs which had their odor-evoked activity increased by each modulator individually, the integrated effects of 5-HT and DA were sometimes non-linear C-D: Dopamine increases odor-evoked firing rate of an AL PN. Bath application of DA increases firing rate of PN relative to control saline before and after (1-way ANOVA, Friedman test, Dunn's multiple comparison, Saline 1 to DA: $p<0.001$; DA to Saline 2: $p<0.0001)$. E-F: Serotonin increases odor-evoked firing rate of the same PN (1-way ANOVA, Friedman test, Dunn's multiple comparison, Saline 1 to $5-\mathrm{HT}$ : $\mathrm{p}<0.0001 ; 5-\mathrm{HT}$ to Saline 2: $p<0.0001)$. G-H: Integrated effects of 5-HT and DA on the same PN are non-linear. Simultaneous bath application of 5-HT and DA increases firing rate of the same PN relative to control saline (1-way ANOVA, Friedman test, Dunn's multiple comparison, Saline 1 to $5-H T+D A: p<0.0001 ; 5-$ HT+DA to Saline 2: n.s) 
A.

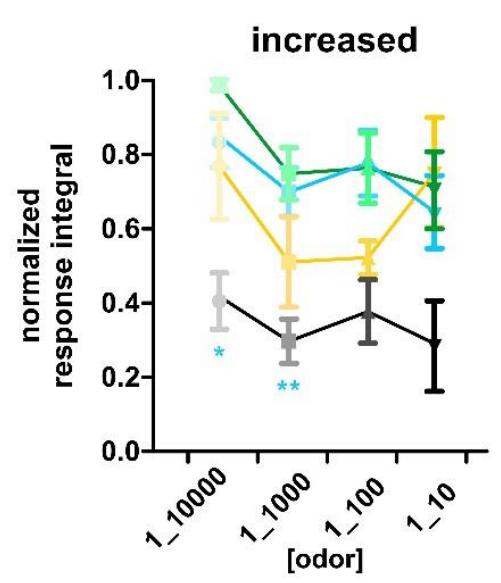

B.

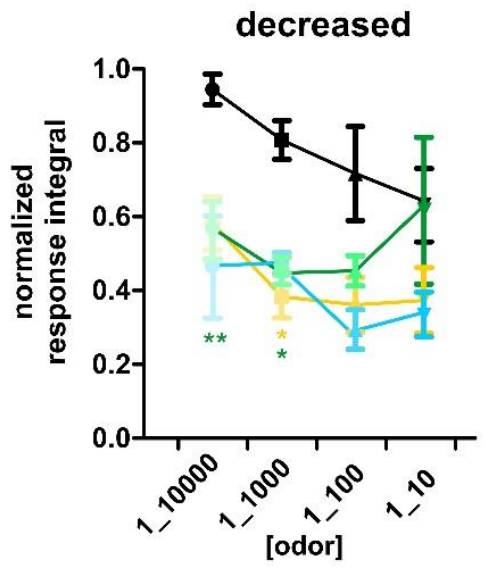

C.

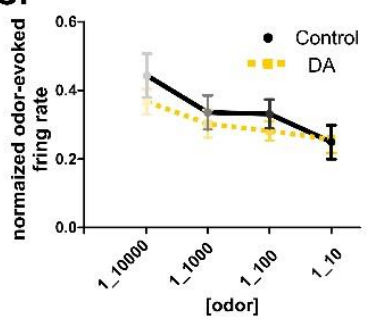

D.

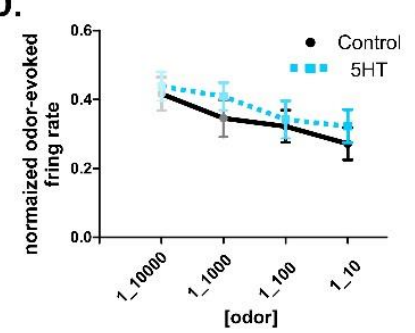

E.

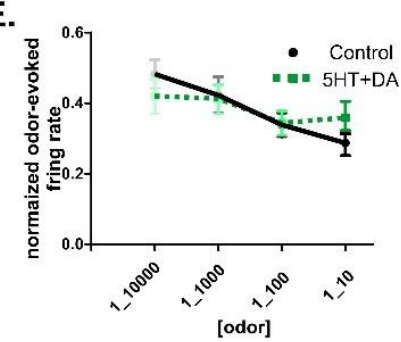

Figure 3: 5-HT and DA exhibit variable effects on PNs

A: $5-\mathrm{HT}$ alone increases response integral in a subset of PNs. Bath application of $5-\mathrm{HT}$ increased response integral at [1:10,000], and [1:1000] Datura blend odor concentration (Kruskall-Wallis test, Dunn's multiple comparison test, [1:10,000], $p<0.01$, [1:1000], $p<0.001$, all other [odor] n.s.), [odor]= odor concentration, control (black), 5-HT (blue), DA (yellow), 5-HT+DA (green). Color intensity increases with [odor]. DA and 5-HT+DA did not significantly increase response integral in these PNs (Kruskall-Wallis test, Dunn's multiple comparison test, all [odor] n.s.) B: In another subset of PNs, DA decreased response integral of PNs at low [odor] (Kruskall-Wallis test, Dunn's multiple comparison test, [1:1000], $\mathrm{p}<0.01$, all other [odor] n.s.). 5-HT+DA decreased this subset of $\mathrm{PN}$ response integrals at low [odor] (Kruskall-Wallis test, Dunn's multiple comparison test, $[1: 10,000], p<0.001,[1: 1000], p<0.01$, all other [odor] n.s.). While there was a trend for 5-HT to decrease response integral in this subset of PNs, 5-HT did not significantly decrease response integral in these PNs (Kruskall-Wallis test, Dunn's multiple comparison test, all [odor] n.s.). E: DA generally decreases firing rate across all $\mathrm{PNs}$ but does not significantly alter response gain (ANCOVA: $\mathrm{F}=$ $0.449194, \mathrm{DFn}=2, \mathrm{DFd}=174, \mathrm{P}=0.6389) \mathrm{F}: 5-\mathrm{HT}$ increases average firing rate across all PNs but does not significantly alter response gain (ANCOVA: $F=$ $0.392765, D F n=2, D F d=186, P=0.6757) G: 5-H T+D A$ have non-linear effects on firing rate of $\mathrm{PNs}$. While, there was a trend for DA to generally decrease odorevoked firing rate, and for $5-\mathrm{HT}$ to generally increase odor evoked firing rate across all PNs, there was no significant change to the slope for either DA, 5-HT or both modulators (ANCOVA: $\mathrm{F}=2.34048, \mathrm{DFn}=2, \mathrm{DFd}=174, \mathrm{P}=$ 0.09931).Therefore, in the subset of PNs recorded, there was no detectable change in gain by any modulator. 
A.

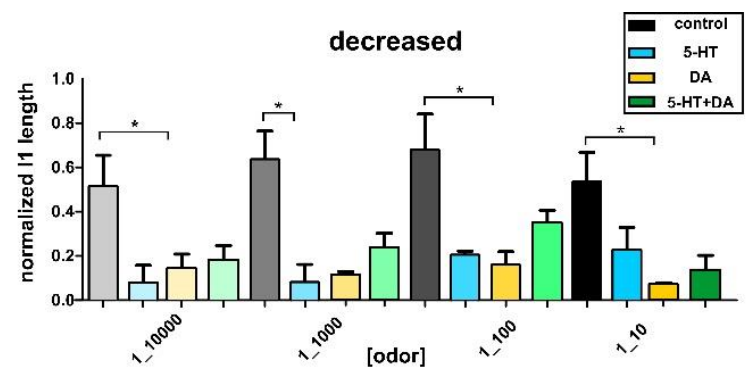

B.

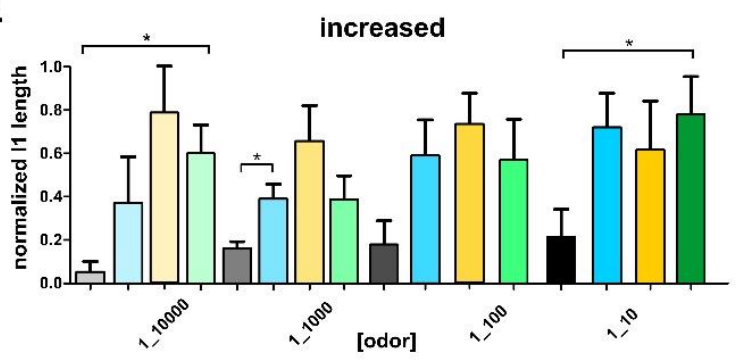

E.

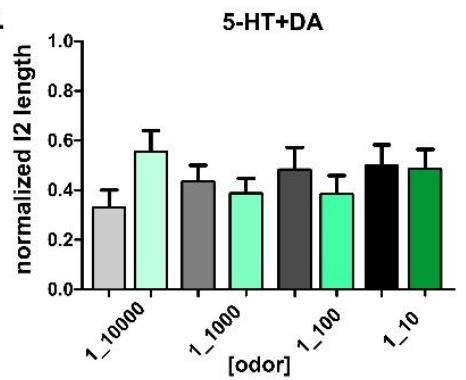

H.

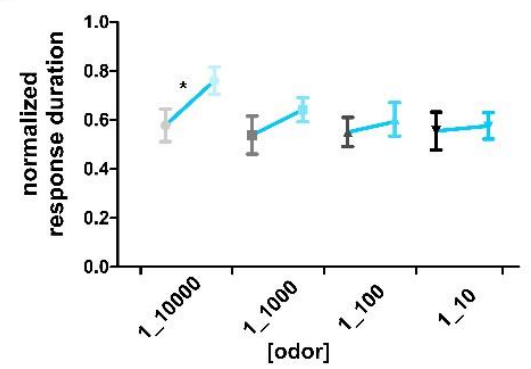

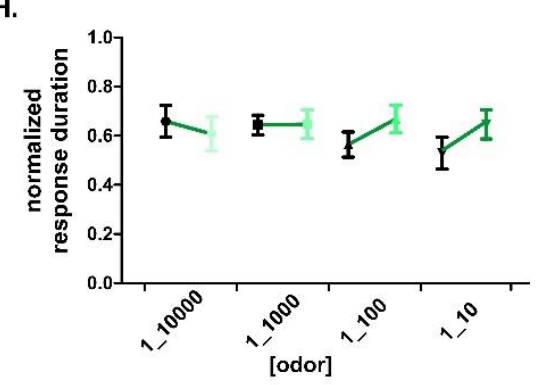

Figure 4: 5-HT and DA modulate PNs via distinct mechanisms and their integrated effects are non-linear

A-B: Both modulators increase and decrease the I1 phase intrinsic to PNs. A: DA significantly decreased the length of I1 at multiple odor concentrations (Kruskall-Wallis test, Dunn's multiple comparison test, [1:10,000], $p<0.05 ;$ [1:1000], n.s.; [1:100], $p<0.05 ;[1: 10]$, $\mathrm{p}<0.05$.) $5-\mathrm{HT}$ significantly decreased I1 length at one odor concentration (Kruskall-Wallis test, Dunn's multiple comparison test, [1:1000], p<0.05.; all other [odor] n.s.), [odor]= odor concentration, control (black), 5-HT (blue), DA (yellow), 5-HT+DA (green). B: 5-HT also significantly increased I1 in a different subset of PNs at [1:1000] (Kruskall-Wallis test, Dunn's multiple comparison test, [1:1000], $p<0.05$.; all other [odor] n.s.). The integrated effects of 5-HT and DA increased the length of I1 at the highest and lowest odor concentrations (Kruskall-Wallis test, Dunn's multiple comparison test, [1:10,000], $p<0.05$.; [1:10], $p<0.05$.; all other [odor] n.s.) C: DAdecreases 12 phase intrinsic to PNs at the highest odor concentration (1-way ANOVA, Bonferonni's multiple comparison test: [1:10], $p<0.0001$; all other [odor] n.s.) D: 5-HT has no effect on I2 (1-way ANOVA, Bonferroni's multiple comparison test; all [odor] n.s). E: Decrease of I2 by DA is not maintained when both modulators are integrated by the same PN (1-way ANOVA, Friedman test, Dunn's multiple comparison test; all [odor] n.s). F: Across all PNs, DA does not significantly alter response duration (1-way ANOVA, Bonferroni's multiple comparison test, n.s. across all [odor]) G: At low concentrations of odor, 5-HT significantly increases the response duration of PNs. At [1:10,000], 5-HT increases response duration across all PNs (1-way ANOVA, Bonferroni's multiple comparison test, $[1: 10,000], p<0.05$, all other [odor] n.s.) H: 5-HT and DA together do not alter response duration. The 5-HT mediated increase in response duration was lost when both modulators were applied together (1-way ANOVA, Bonferroni's multiple comparison test, n.s. across all [odor]). 
A.

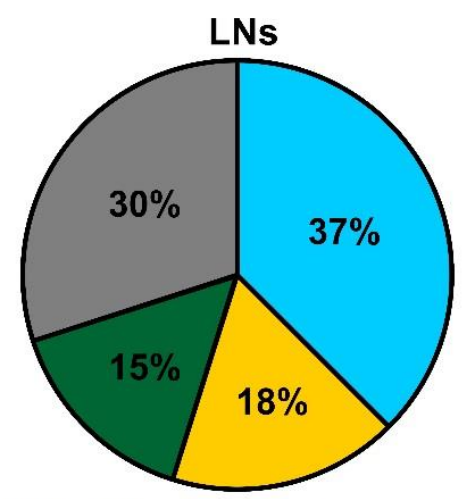

5HT DA 5HT+DA unaffected
B.

odor evoked firing rate LNs

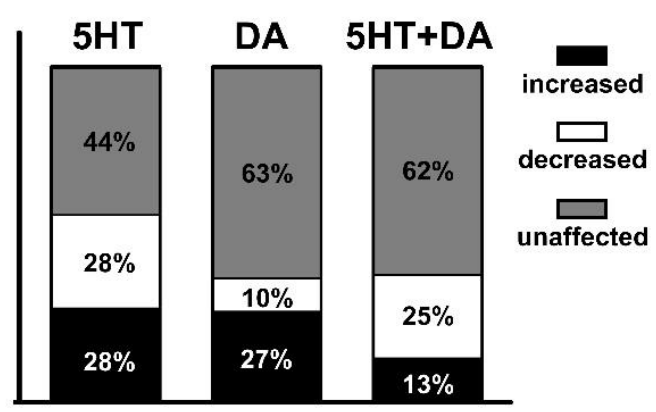

C.
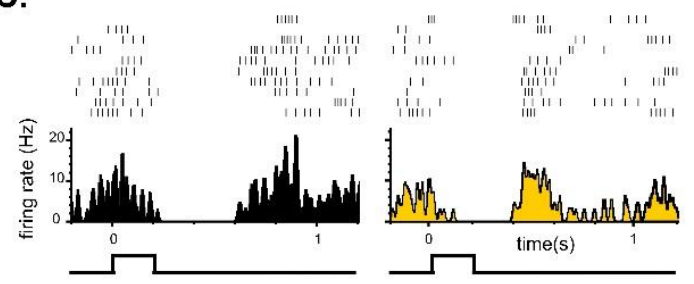

E.
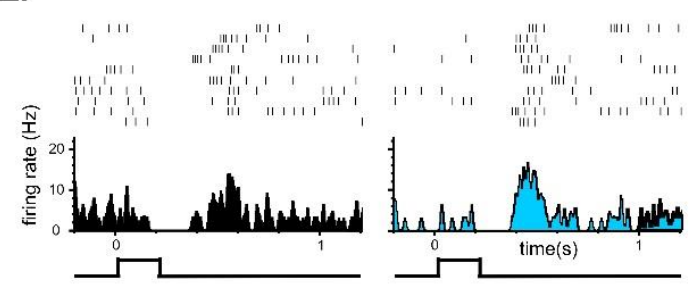

G.
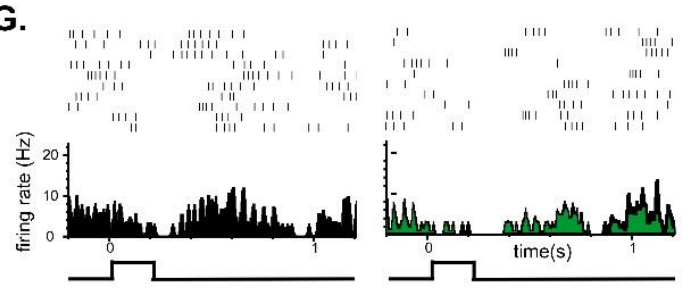
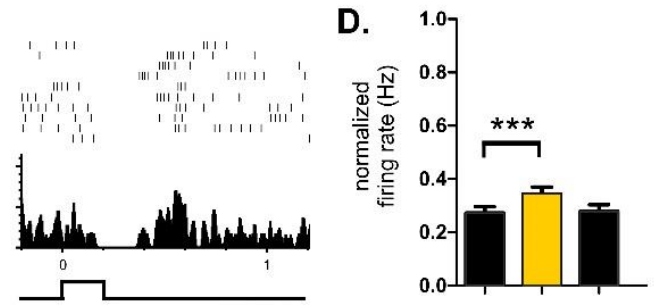

F.

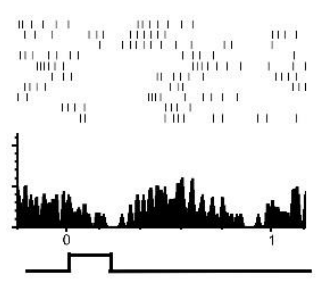

Figure 5: 5-HT and DA modulate odor-evoked firing rate of local interneurons.

A: Most LNs were selectively modulated by $5-\mathrm{HT}$ alone. The largest proportion of total LNs $(37 \%)$ had their odor-evoked firing rate affected by $5-\mathrm{HT}$ alone (Figure $5 \mathrm{H}$ ). Subsets of LNs were selectively modulated by DA alone $(18 \%)$, both modulators $(15 \%)$, and a large subset had their odor-evoked firing rate unaffected (Figure $5 \mathrm{H}$ ). This suggests that while some LNs integrate the influence of both $5-\mathrm{HT}$ and DA, more LNs are selectively modulated by $5-\mathrm{HT}$

B: Both 5-HT and DA exhibit variable effects on firing rate. Each modulator is capable of either increasing or decreasing odor-evoked firing rate during pure excitation or rebound excitation depending on the LN. C-D: DA increases rebound excitation. In an individual LN, DA increases rebound excitation (1-way ANOVA, Dunn's multiple comparison test, control saline to DA, $p<0.0001$; DA to control saline, not significant (n.s.)). E-F: 5-HT increases rebound excitation in the same LN (1-way ANOVA, Bonferroni's multiple comparison test, control saline to $5-\mathrm{HT} p<0.001 ; 5-\mathrm{HT}$ to control saline, $\mathrm{p}<0.0001)$. G-H: In the same LN, the integrated effects of both 5-HT and DA resulted in decreased rebound excitation (1-way ANOVA, Bonferroni's multiple comparison test, control saline to 5-HT+DA, $p<0.0001 ; 5-$ HT+DA to control saline, n.s.). 
A.

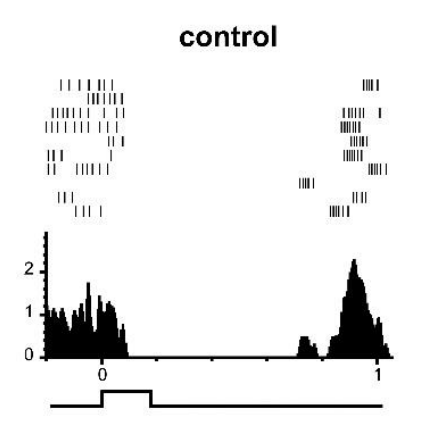

C.

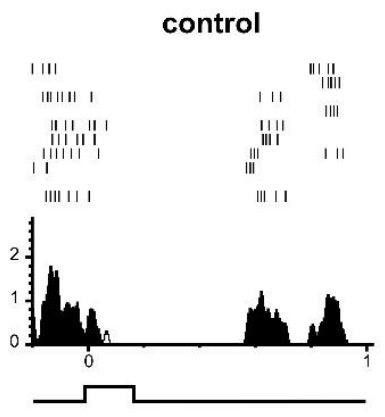

E.

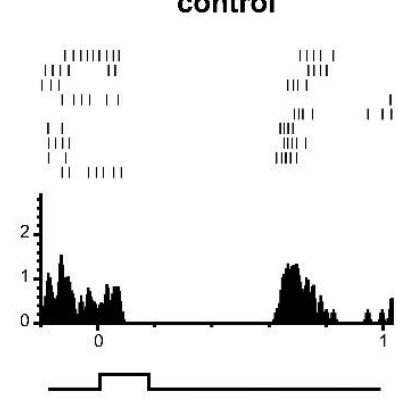

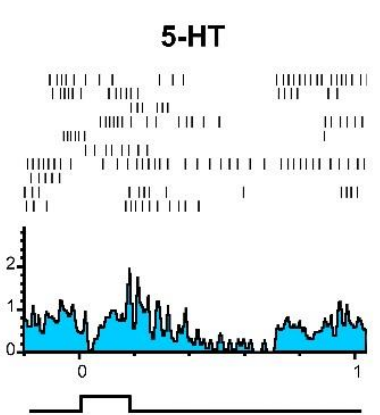

DA

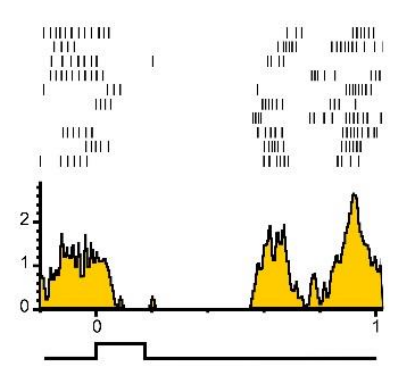

5-HT+DA
B.

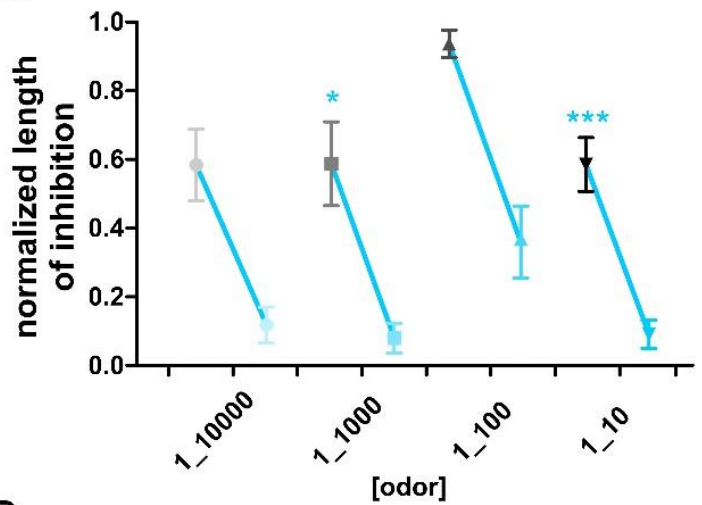

D.

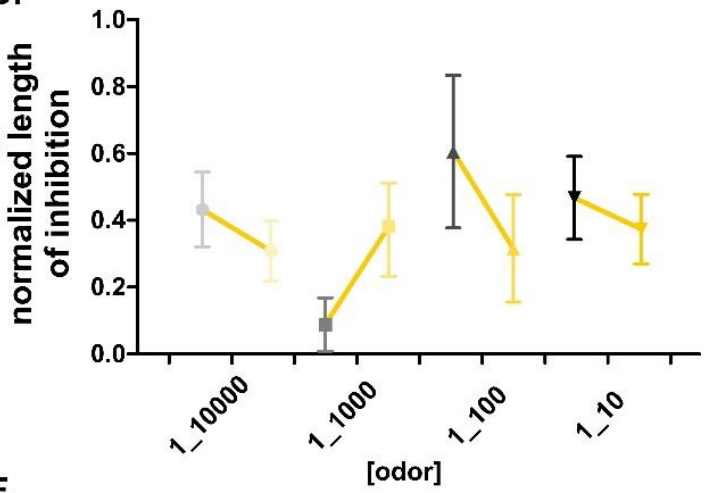

F.

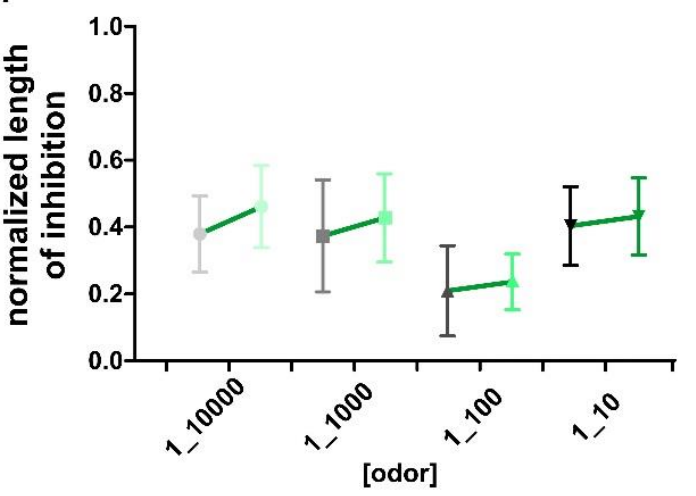

Figure 6: 5-HT decreases length of odor-evoked inhibition in local interneurons

A: $5-\mathrm{HT}$ alone decreases odor-evoked inhibition in LNs. B: At [1:1000] and [1:10], 5-HT significantly decreased the length of odor-evoked inhibition across a subset of PNs (Kruskill-Wallis test, Dunn's multiple comparison test, [1:1000], $\mathrm{p}<0.01$; [1:10], $\mathrm{p}<0.0001$; all other [odor] n.s.), [odor]= odor concentration, control (black), 5-HT (blue), DA (yellow), 5-HT+DA (green). C: In the same PN, DA does not alter odor-evoked inhibition. D: There were no significant changes to odor-evoked inhibition by DA at any odor concentration in the same subset of PNs from B (Kruskill-Wallis test, Dunn's multiple comparison test, all [odor] n.s.) E: 5-HT and DA do not significantly alter the length of odor-evoked inhibition in the same PN. F: The 5-HT mediated decrease in inhibition is lost when both modulators are applied simultaneously to the same subset of PNs (Kruskill-Wallis test, Dunn's multiple comparison test, all [odor] n.s.) 
A.

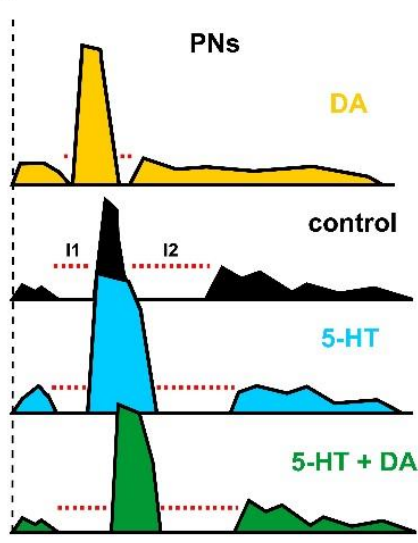

B.

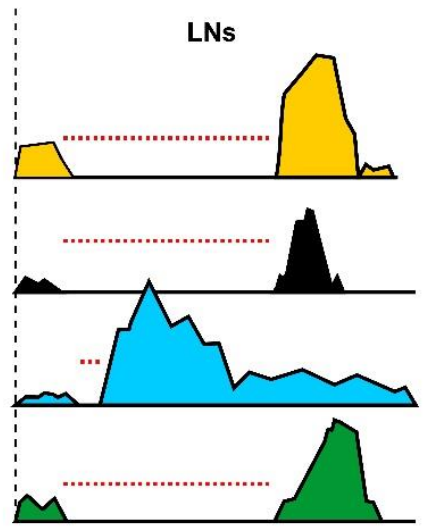

C.

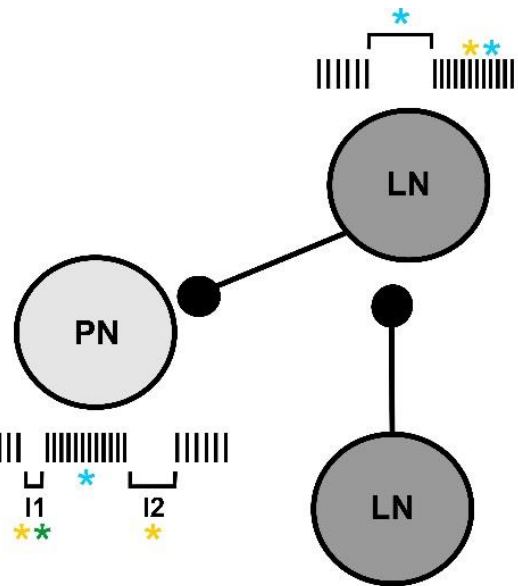

**||||||||||||||||||||||||

Figure 7: DA modulates response timing, while 5-HT alters lateral interactions of LNs to increase sensitivity and response duration of PNs. A: DA modulates response timing of AL neurons, while 5-HT modulates sensitivity. Across all PNs, DA significantly reduces the length of $\mathrm{I} 1$ and $\mathrm{I} 2$, reducing the amount of time it takes for PNs to return to baseline. 5-HT significantly increases the response duration of PNs at low odor concentrations, increasing the time PNs can respond. B: 5-HT selectively modulates LNs to alter inhibitory drive onto PNs and other LNs. A large proportion of $\mathrm{LNs}$ are modulated selectively by $5-\mathrm{HT}$, increasing odor-evoked firing rate, and decreasing odorevoked inhibition of LNs. While DA increases also increases rebound excitation in LNs, this effect is likely mediated by direct modulation of channels intrinsic to PNs. C: Summary. PNs respond to odors with stereotyped, I1-E-I2 phase, while LNs exhibit either odor-evoked excitation or inhibition. The arrows in subsequent panels represent whether a specific feature or neuron type was decreased or increased by either modulator. DA alone decreases the length of I1 and I2, allowing PNs to respond to odors more quickly and rapidly recover to baseline. 5-HT selectively increases the odor-evoked firing rate of the highest proportion of LNs, leading to complex changes in lateral interactions. While some LNs have their odor-evoked activity decreased or increased by the simultaneous presence of both modulators, DA masks the 5$\mathrm{HT}$ mediated effects on response duration, and the DA specific decrease on 12 is lost. This suggests that the integrated effects may be nonlinear. 


\section{CHAPTER 4}

Systematic analysis of transmitter co-expression reveals organizing principles of olfactory local interneuron heterogeneity 


\section{Summary:}

Historically, neurons were categorized based on morphology or location within the network. However, each neuronal class can be surprisingly heterogeneous in their synaptic, biophysical and transcriptional profiles (Cohen et al., 2015; Eddine et al., 2015; Okaty et al., 2015; Li et al., 2017). This is true across species and brain regions, leading to the identification of numerous LN subtypes within cortex (Flames and Marín, 2005; DeFelipe et al., 2013; Yavorska and Wehr, 2016), hippocampus (Maccaferri and Lacaille, 2003), and spinal cord (Gabitto et al., 2016; Sweeney et al., 2018). The parameters used to sub-categorize neurons are often partially overlapping across sub-types. For example, two spinal interneuron populations, which support different motor output (limb vs. thoracic) can be distinguished, and further subdivided, based on transcription factor expression profile (Sweeney et al., 2018). Similarly, 13 distinct groups of GABAergic cortical interneurons exhibit partially overlapping expression of multiple neuropeptides and modulators (Gonchar et al., 2007). Thus, attempting to assign a unified role to a population of neurons based on single markers is misleading. So how do we make sense of this heterogeneity?

To determine the organizing principles that govern neuronal heterogeneity it is critical to use a combinatorial approach, which takes multiple parameters, like transmitter co-expression, into consideration. Here, I take advantage of the numerically simple insect olfactory system to make sense of within cell class heterogeneity. Much of the computational capacity of the olfactory system is mediated by LNs that refine the transfer of information between ORNs and PNs. Local interneurons (LNs) are the main source of intrinsic modulation in the antennal lobe and perform many tasks including presynaptic gain control, divisive normalization, lateral excitation and inhibition, and dynamic control of response range (reviewed in Martin et al., 2011 and Wilson et al, 2013). LNs are particularly heterogeneous and the insect olfactory system is no exception (Nässel and Homberg, 2006). As a population, insect LNs are heterogeneous in their morphology, physiology (Chou et al., 2010; Seki et al., 2010; Reisenman et al., 2011), and transmitter coexpression (Homberg et al., 1990; Berg et al., 2007; Utz et al., 2008; Carlsson et al., 2010; Siju et al., 2013). Furthermore, in M. sexta, as well as other insects, AL LNs express a combination of GABA and multiple neuropeptides (Homberg et al., 1990; Schachtner et al., 2004; Utz et al., 2007; Utz et al., 2008; Reisenman et al., 2011; Fusca et al., 2015). However, it is unknown if certain 
combinations of neuropeptides are consistently co-expressed together, and whether co-expressed peptides target different populations of neurons due to differences in receptor expression. Furthermore, there are no correlations between morphology, physiology and GABA expression in M. sexta LNs (Reisenman et al., 2011) suggesting a high degree of heterogeneity within this population. Consequently, understanding the functional roles of individual LNs is complex as we lack a systematic analysis of transmitter co-expression (Nässel, 2018).

In this chapter, I identify the organizing principles of transmitter co-expression in $M$. sexta LNs. Using immunocytochemistry and computational modeling, I take a combinatorial approach to determine the organizing principles of neurotransmitter co-expression in a diverse population of local interneurons. I first systematically determine the co-localization of neuropeptides and GABA in LNs and find that single olfactory LNs co-express the small neurotransmitter, GABA and neuropeptides from at least four families. Neuropeptides were heterogeneously co-expressed across the entire population. My results argue against clear-cut definitions of neuron subpopulations based on the expression of single neuropeptides. Additionally, the heterogeneity of transmitter co-expression in AL LNs can be explained in modeling by a few specific co-expression relationships. This suggested that specific co-expression relationships may shape heterogeneity in LNs. Finally, to determine the potential consequences of peptide release from LNs, I assessed neuropeptide receptor expression across the principal neuron types of the antennal lobe (ORNs, PNs and LNs). I identified the populations of neurons that express neuropeptide and GABAb receptors and found that all receptors were expressed by input, output and local processing neurons in the AL. By determining the consistency with which LNs co-express multiple modulators as well the functional AL neuron classes that express the receptors for these modulators, I determined which modulators are likely released under the same context (likely the degree of network activity) and whether they target the same population of neurons. I found that receptors for all neuropeptides and GABA were expressed by the input, output and local processing cells within the AL. Overall, my results suggest that activation of olfactory LNs results in a dynamic cocktail of modulators that have the potential to influence every level of olfactory processing within the AL (Lizbinski et al., 2018). Broadly, this work provides 1) an atlas of heterogeneous transmitter co-expression in AL local interneurons 2) putative neuropeptide receptor/GABAb receptor distribution across the functional neuron types of the olfactory system and finally 3) an analytical tool to assess heterogeneity in any neural population. 


\section{Works Cited:}

Berg, B. G., Schachtner, J., Utz, S., and Homberg, U. (2007). Distribution of neuropeptides in the primary olfactory center of the heliothine moth Heliothis virescens. Cell Tissue Res. 327, 385-398. doi: 10.1007/s00441-006-0318-x

Carlsson, M. A., Diesner, M., Schachtner, J., and Nässel, D. R. (2010). Multiple neuropeptides in the Drosophila antennal lobe suggest complex modulatory circuits. J. Comp. Neurol. 518, 33593380. doi: $10.1002 / \mathrm{cne} .22405$

Chou, Y. H., Spletter, M. L., Yaksi, E., Leong, J. C., Wilson, R. I., and Luo, L. (2010). Diversity and wiring variability of olfactory local interneurons in the Drosophila antennal lobe. Nat. Neurosci. 13, 439-449. doi: 10.1038/nn.2489

Cohen JY, Amoroso MW, Uchida N (2015) Serotonergic neurons signal reward and punishment on multiple timescales. eLife 4:e06346 10.7554/eLife.06346

DeFelipe J, et al. . (2013) New insights into the classification and nomenclature of cortical GABAergic interneurons. Nat Rev Neurosci14:202-216. 10.1038/nrn3444

Eddine R, Valverde S, Tolu S, Dautan D, Hay A, Morel C, Cui Y, Lambolez B, Venance L, Marti F, Faure $\mathrm{P} \quad$ (2015) A concurrent excitation and inhibition of dopaminergic subpopulations in response to nicotine. Scientific Reports 5:8184. 10.1038/srep08184

Flames N, Marín O (2005) Developmental mechanisms underlying the generation of cortical interneuron diversity. Neuron 46:377-381. 10.1016/j.neuron.2005.04.020

Fusca, D., Schachtner, J., and Kloppenburg, P. (2015). Colocalization of allatotropin and tachykininrelated peptides with classical transmitters in physiologically distinct subtypes of olfactory local interneurons in the cockroach (Periplaneta americana). J. Comp. Neurol. 523, 1569-1586. doi: $10.1002 /$ cne. 23757

Gabitto MI, Pakman A, Bikoff JB, Abbott LF, Jessell TM, Paninski L (2016) Bayesian sparse regression analysis documents the diversity of spinal inhibitory interneurons. Cell 165:220-233. 10.1016/j.cell.2016.01.026

Gonchar Y, Wang Q, Burkhalter A (2007) Multiple distinct subtypes of GABAergic neurons in mouse visual cortex identified by triple immunostaining. Front Neuroanat $1: 3$ 10.3389/neuro.05.003.2007

Homberg, U., Kingan, T. G., and Hildebrand, J. G. (1990). Distribution of FMRFamide-like immunoreactivity in the brain and suboesophageal ganglion of the sphinx moth Manduca sexta and colocalization with SCPB-, BPP-, and GABA-like immunoreactivity. Cell Tissue Res. 259,

401- 419. doi: 10.1007/bf01740767

Li H, Horns F, Wu B, Xie Q, Li J, Li T, Luginbuhl DJ, Quake SR, Luo L (2017) Classifying Drosophila olfactory projection neuron subtypes by single-cell RNA sequencing. Cell 171:1206-1220.e1222.

Lizbinski, K. M., Marsat, G., \& Dacks, A. M. (2018). Systematic Analysis of Transmitter Coexpression Reveals Organizing Principles of Local Interneuron Heterogeneity. eNeuro, 5(5). 
Maccaferri G, Lacaille JC (2003) Interneuron diversity series: Hippocampal interneuron classificationsmaking things as simple as possible, not simpler. Trends Neurosci 26:564-571.

10.1016/j.tins.2003.08.002

Nässel, D. R., \& Homberg, U. (2006). Neuropeptides in interneurons of the insect brain. Cell and tissue research, 326(1), 1 .

Nässel, D. R. (2018). Substrates for neuronal cotransmission with neuropeptides and small molecule neurotransmitters in Drosophila. Frontiers in cellular neuroscience, 12, 83.

Okaty BW, Freret ME, Rood BD, Brust RD, Hennessy ML, deBairos D, Kim JC, Cook MN, Dymecki

SM (2015) Multi-scale molecular deconstruction of the serotonin neuron system. Neuron 88:774791. 10.1016/j.neuron.2015.10.007

Reisenman, C. E., Dacks, A. M., and Hildebrand, J. G. (2011). Local interneuron diversity in the primary olfactory center of the moth Manduca sexta. J. Comp. Physiol. A Neuroethol. Sens. Neural Behav. Physiol. 197, 653-665. doi: 10.1007/s00359-011-0625-x

Schachtner, J., Trosowski, B., D'Hanis, W., Stubner, S., \& Homberg, U. (2004). Development and steroid regulation of RFamide immunoreactivity in antennal-lobe neurons of the sphinx moth Manduca sexta. Journal of experimental biology, 207(14), 2389-2400.

Seki, Y., Rybak, J., Wicher, D., Sachse, S., and Hansson, B. S. (2010). Physiological and morphological characterization of local interneurons in the Drosophila antennal lobe. J. Neurophysiol. 104, 1007-1019. doi: 10.1152/jn.00249.2010

Utz, S., Huetteroth, W., Vömel, M., and Schachtner, J. (2008). Mas-allatotropin in the developing antennal lobe of the sphinx moth Manduca sexta: distribution, time course, developmental regulation, and colocalization with other neuropeptides. Dev. Neurobiol. 68, 123-142. doi: 10.1002/dneu.20579

Utz, S., Huetteroth, W., Wegener, C., Kahnt, J., Predel, R., \& Schachtner, J. (2007). Direct peptide profiling of lateral cell groups of the antennal lobes of Manduca sexta reveals specific composition and changes in neuropeptide expression during development. Developmental neurobiology, 67(6), 764-777.

Yavorska I, Wehr M (2016) Somatostatin-expressing inhibitory interneurons in cortical circuits. Front Neural Circ 10:76. 10.3389/fncir.2016.00076 
(this chapter is taken directly from my publication "Lizbinski, K. M., Marsat, G., \& Dacks, A. M. (2018). Systematic Analysis of Transmitter Coexpression Reveals Organizing Principles of Local Interneuron Heterogeneity. eNeuro, 5(5).")

Abstract: Broad neuronal classes are surprisingly heterogeneous across many parameters and subclasses often exhibit partially overlapping traits including transmitter co-expression. However, the extent to which transmitter co-expression occurs in predictable, consistent patterns is unknown. Here, we demonstrate that pairwise co-expression of GABA and multiple neuropeptide families by olfactory local interneurons (LNs) of the moth Manduca sexta is highly heterogeneous, with a single LN capable of expressing neuropeptides from at least four peptide families and few instances in which neuropeptides are consistently co-expressed. Using computational modeling, we demonstrate that observed co-expression patterns cannot be explained by independent probabilities of expression of each neuropeptide. Our analyses point to three organizing principles that once taken into consideration allow replication of overall co-expression structure: 1peptidergic neurons are highly likely to co-express GABA; 2- expression probability of Allatotropin depends upon Myoinhibitory peptide expression; 3- the all-or-none co-expression patterns of Tachykinin neurons with several other neuropeptides. For other peptide pairs, the presence of one peptide was not predictive of the presence of the other and co-expression probability could be replicated by independent probabilities. The stochastic nature of these coexpression patterns highlights the heterogeneity of transmitter content among LNs and argues against clear-cut definition of subpopulation types based on the presence of single neuropeptides. Furthermore, the receptors for all neuropeptides and GABA were expressed within each population of principal neuron type in the AL. Thus, activation of any given LN results in a dynamic cocktail of modulators that have the potential to influence every level of olfactory processing within the AL.

Significance Statement: Understanding the functional roles of individual local interneurons (LNs) is complex because traits, like transmitter co-expression, are often partially overlapping across the population. Here, we find that single olfactory LNs co-express neuropeptides from at least four individual peptide families, and that GABA and neuropeptides are partially and heterogeneously co-expressed across the entire population. The stochastic nature of many observed co-expression patterns argues against clear-cut and exclusive definition of subpopulations based on the expression of single neuropeptides. Overall, our results suggest that activation of any given LN 
causes the release of a variable combination of neuropeptides and GABA that, based on receptor expression, target the input, output and local processing stages of olfactory coding.

\section{Introduction:}

The historical concept of a cell-type, propelled by the work of Cajal and Golgi, suggests that distinct functional classes of neurons can be identified based on their morphology. Yet recent advances in transcriptomics and electrophysiology have revealed that even neurons within a single cell-type can still be surprisingly heterogeneous in their synaptic, biophysical and transcriptional profiles (Cohen et al., 2015; Eddine et al., 2015; Okaty et al., 2015; Li et al., 2017). Local interneurons (LNs) tend to be particularly heterogeneous across many parameters, leading to the identification of numerous LN subtypes within cortex (Flames and Marin, 2005; DeFelipe et al., 2013; Yavorska and Wehr, 2016), hippocampus (Maccaferri and Lacaille, 2003) and spinal cord (Gabitto et al., 2016; Sweeney et al., 2018). For example, two spinal interneuron populations, which support different motor output (limb vs. thoracic) can be distinguished, and further subdivided, based on transcription factor expression profile (Sweeney et al., 2018). Similarly, 13 distinct groups of GABAergic cortical interneurons exhibit partially overlapping expression of multiple neuropeptides and modulators (Gonchar et al., 2007). Thus, parameters used to classify LN sub-populations can be partially overlapping across functionally distinct sub-populations. Consequently, attempting to assign a unified functional role to sub-populations based on single molecular markers or transmitters is misleading. How then, do we reconcile heterogeneity within cell-types?

To determine the organizing principles that govern neuronal heterogeneity it is critical to use a combinatorial approach, which takes multiple parameters, like transmitter co-expression, into consideration. The insect antennal lobe (AL), analogous to the olfactory bulb, is an excellent system in which to approach this problem due to the wealth of information on local interneuron physiology, morphology and transmitter content combined with its relative numerical simplicity. The olfactory system detects and transforms odor input into meaningful output ultimately informing an animal's choice to mate, seek food, or avoid predators (Ache and Young, 2005). Odorants are first detected by olfactory receptor neurons (ORNs) which synapse onto projection neurons (PNs) within sub-structures called glomeruli that form an odor-topic map within the AL. The input/output relationship between ORNs and PNs is refined by a diverse population of LNs 
that transform odor information via a variety of mechanisms (Wilson, 2013). In Manduca, LNs are primarily inhibitory (Christensen et al., 1993), broadly tuned to odorants, exhibit both inhibitory and excitatory responses, and are highly morphologically and physiologically diverse (Hildebrand et al., 1992; Reisenman et al., 2011). However, there are no correlations between morphology, physiology and GABA expression in Manduca LNs (Reisenman et al., 2011) suggesting a high degree of heterogeneity within this population. Furthermore, in Manduca, as well as other insects, AL LNs express a combination of GABA and multiple neuropeptides (Homberg et al., 1990; Schachtner et al., 2004; Utz et al., 2007; Utz et al., 2008; Reisenman et al., 2011; Fusca et al., 2015). Consequently, understanding the functional roles of individual LNs is complex as we lack a systematic analysis of transmitter co-expression (Nässel, 2018).

We used the olfactory system of Manduca to determine if sub-classes of LNs have common transmitter profiles. We characterized each pair-wise co-expression pattern for GABA and five neuropeptides, and found that although almost all peptidergic LNs co-express GABA, neuropeptide co-expression is heterogeneous across LNs. Using computational modeling, we demonstrate that many co-expression patterns cannot be explained by independent probabilities of expression of each peptide, highlighting that certain pairs of peptides co-occur more (or less) often than by chance. For other pairs, the presence of one peptide was not predictive of the presence of the other, and co-expression probability could be replicated by independent probabilities. The stochastic nature of these co-expression patterns highlights the heterogeneity of transmitter content among LNs and argues against clear-cut and exclusive definition of subpopulation types based on the presence of a single neuropeptide. One possible explanation for this heterogeneity is that principal cell classes within the AL express different GABA and neuropeptide receptors. This would segregate the influence of each modulator across different cell types (Nusbaum et al., 2001; Tritsch et al., 2016; Nusbaum et al., 2017) as is the case for the clock network of Drosophila (Liang et al., 2017). However, this is not likely to be the case here, as all neuropeptide and $\mathrm{GABA}_{\mathrm{B}}$ receptors were expressed within every cell class of the AL (ORNs, PNs and LNs). Overall, our results suggest that activation of any given LN likely releases a variable combination of peptides and GABA to potentially influence every cell class within the AL. 


\section{Materials and Methods:}

Animals: Manduca sexta were raised at West Virginia University as previously described (Bell and Joachim, 1976; Daly et al., 2013). Equal numbers of unmated adult males and female moths were pooled for all data.

Immunocytochemistry: Brains were dissected in physiological saline (Christensen and Hildebrand, 1987), fixed in $4 \%$ paraformaldehyde overnight at $4{ }^{\circ} \mathrm{C}$, embedded in $5 \%$ agarose to be sectioned at $100 \mu \mathrm{m}$ using a Leica VT $1000 \mathrm{~S}$ vibratome. Sections were washed in PBS with $1 \%$ triton X-100 (PBST), blocked in PBST and 2\% IgG free BSA (Jackson Immunoresearch; Cat\#001-000-161) and then incubated in blocking solution with $5 \mathrm{mM}$ sodium azide and primary antibodies. For all rabbit-neuropeptide/mouse-GABA protocols, sectioned tissue was incubated for 48 hours at dilutions of 1:3000 and 1:500 respectively. Sections were then briefly washed with PBS, PBST, cleared with ascending glycerol washes and then mounted on slides with Vectashield (Vector Laboratories; Cat\#H-1000). All neuropeptide antibodies used in this study were raised in rabbit. For protocols in which we labeled with two antisera raised in rabbit, we used APEX Antibody Labeling Kits 488, 555, 647 (Invitrogen;Cat \#s A10468, A10470, A10475, respectively) to directly attach a fluorophore with excitation/emission spectra at different wavelengths to each primary to avoid cross-labeling from a secondary antibody (Bradley et al., 2016). Using the resin tip from the APEX kit, a small amount of the antibody (10-20 $\mu$ g) was pushed through the resin using an elution syringe and the reactive dye was prepared using DMSO and a labeling buffer (Solutions provided in APEX kit). The reactive dye was eluted through the tip onto the antibody remaining in the resin to covalently bond the fluorescent label to the $\mathrm{IgG}$ antibodies. The tip was incubated overnight $4{ }^{\circ} \mathrm{C}$ or at room temperature for 2 hours and the labeled product was eluted through the tip. Resulting labeled antibody volume of 50uL in a total volume of 2400ul was used to label 6 brains at equal dilution of 400ul per well and incubated for 72 hours in 3\% triton X-100 with PBSAT. Sections were then washed and mounted as above.

Antibody Characterization: Specificity controls (including pre-adsorption controls) for the ASTA, Mas-AT, TK, and MIP antibodies in Manduca brain tissue are described completely in (Lizbinski et al., 2016). GABA pre-adsorption controls in Manduca AL tissue for the mouse GABA antiserum are described in (Bradley et al., 2016). The antibodies used in this study likely cross-react with several isoforms within the same peptide family. Thus, our results can only resolve principles at the level of peptide family and not individual peptide isoforms. 
GABA- The GABA antibody (Sigma Aldrich, cat \# A2052) was raised in rabbit against GABA coupled to BSA with paraformaldehyde.

MIP- Antiserum raised in rabbit against MIP conjugated to thyroglobulin was produced by $\mathrm{M}$. Eckert, Jena Germany and provided by C. Wegener, Marburg Germany (Predel et al., 2001). (RRID: AB_2314803).

Mas-AT -Antiserum raised in rabbit against Manduca allatotropin (Mas-AT) was kindly provided by Dr. J. Veenstra, University of Bordeaux, Talence, France; (Veenstra and Hagedorn, 1995). (RRID: AB_2313973)

AST-A- Antiserum was raised (Reichwald et al., 1994) in rabbit against octadecapeptideallatostatin (Pratt et al., 1991), ASB2, (AYSYVSEYKALPVYNFGL-NH2) of Diploptera punctata and kindly provided by Dr. J. Veenstra, University of Bordeaux, Talence, France. It recognizes AKSYNFGLamide, a form of AST and other AST-like peptides.

TK- Antiserum raised in rabbit against locust tachykinin II with bovine thyroglobulin with glutaraldehyde was kindly provided to us Dr. J. Veenstra, University of Bordeaux, Talence, France. (RRID: AB_2341129)

FMRF- FMRFamide antiserum was raised against synthetic RF-amide coupled to bovine thyroglobulin with glutaraldehyde and provided by Dr. Eve Marder (Marder et al., 1987). Preadsorption controls of the antiserum against synthetic FMRFamide eliminated labeling in larval Manduca nervous tissue (Witten and Truman, 1996).

Confocal Microscopy: Image stacks were scanned using an Olympus Fluoview FV1000 confocal microscope with argon and green and red HeNe lasers. Scans were taken at either 800x800 or $1024 \times 1024$ pixel resolution, 1.5 um between optical sections, using both 20x/0,80 Oil UPlanApo and 40x/1.30 Oil $\infty$ 0.17/FN 26.5, 80um pinhole size, Olympus lenses. Fluoview (FV10-ASW Viewer software(Ver.4.2b)) was also used to set brightness levels and Corel Draw X4 was used to organize figures.

Cell counts and co-expression: Images of immunostained brains were exported as .tiff stacks in Fluoview software. Stacks were then imported into VAA3D software (available at www.vaa3d.org/) (Peng et al., 2010) to determine individual cell counts and co-expression cell counts. The number of local interneurons in the lateral cell cluster that express each transmitter 
were counted in VAA3D ( $\mathrm{n}=6$ brains per label combination, 3 brains per sex). We used cell body size, and location within the lateral cell cluster to distinguish between LNs and PNs (Homberg et al., 1988). The average and standard deviation of number of cells per AL that expressed a given transmitter were calculated for each combination. Wilcoxon rank sum tests were performed using Graph Pad Prism v.5.01 (Graphpad Software Incorporated) to determine if there was any significant difference between the left and the right AL for each brain. Co-expression ratios were determined by dividing the number of cells expressing both an individual neuropeptide and GABA by the total number of cells expressing just the neuropeptide and calculated in Excel. Neuropeptide co-expression ratios were determined in the same manner for every possible pairwise combination using data from peptides stained using the APEX kits. FMRF/MIP co-expression ratios were not calculated as the APEX kits labeled significantly less MIP neurons than all other runs and therefore the ratios would not have reflected accurate co-expression. Thus, FMRF/MIP co-expression was not used in subsequent models or computational analysis as neither a constraint nor a relationship to replicate. All other neuropeptide/neuropeptide co-expression experiments labeled an accurate \# of cell bodies when compared to cell counts from GABA/neuropeptide runs using indirect immunocytochemistry. Cell count totals and standard deviations from APEX kit labeling (Figure 2) were used in all model iterations, as co-expression ratios were calculated using that data.

Putative neuropeptide receptor sequence BLAST: We used receptor sequences from closely related invertebrate species to identify putative sequence homologs on Manduca scaffolds. Protein sequences from Drosophila melanogaster and other closely related species were identified by annotation (see Table 2) and queried against the Manduca genome using tblastn (National Agricultural Library, i5k initiative https://i5k.nal.usda.gov/Manduca_sexta). Top matches to each receptor sequence in Manduca were subsequently queried against the NCBI nr database to confirm their putative annotation as Manduca receptor homologs. These sequences were used for primer design for RT-qPCR analysis of putative neuropeptide receptor expression in the antennae, medial and lateral cell clusters, and brain. Sequences that were previously identified in Manduca for MasATr and RpS3 (Jiang et al., 1996; Horodyski et al., 2011) were downloaded as FASTA files from NCBI (http://www.ncbi.nlm.nih.gov/gene/?term=) and used to design RT-qPCR primers. Open reading frames were established using ORF Finder at http://www.ncbi.nlm.nih.gov/projects/gorf/. A recent study partially annotated the Manduca genome (Kanost et al., 2016). We used the 
Manduca raw sequence, and assembled genome sequence at NCBI Assembly ID GCA_000262585 from Kanost (http://www.ncbi.nlm.nih.gov/assembly/GCA_000262585.1) (Kanost et al., 2016) and identified the sequence IDs for each of the transcripts in question (Table 1). None of the putative receptor sequences are currently annotated in NCBI Assembly ID GCA_000262585.

Primer design: Open reading frame nucleotide sequences for each receptor, as established above, were used as the basis for primer design for RT-qPCR. Primers were designed using http://www.bioinformatics.nl/cgi-bin/primer3plus/primer3plus.cgi and checked for optimal conditions using OligoAnalyzer 3.1 (https://www.idtdna.com/calc/analyzer). Primers and amplicons were then run through a BLAST of the Manduca genome to determine if they matched to the specified sequence and to rule out potential priming mismatches with other parts of the genome. Table 2 lists primer sequences and annealing temperatures. All primers used for RTqPCR amplified a 90-125bp stretch of sequence.

Real Time (RT) qPCR: Antennae, medial cell clusters, lateral cell clusters and brains were collected from 2-6 day old, unmated, naive adult Manduca and RNA was extracted using a TRIzol reagent (Molec. Research center Cat\# \# TR 118). Equal numbers of pooled males and females were used for each biological tissue sample for a total of 3 biological samples for each tissue type ( $n=3$ antennae; $n=40$ medial cell clusters from 20 brains; $n=40$ medial cell clusters from 20 brains; and $\mathrm{n}=2$ brains). We used the 40 s ribosomal protein $\mathrm{s} 3(\mathrm{RpS} 3)$ as our reference gene. RpS3 expression values were consistent across biological replicates. RNA was treated with TURBO DNA-free ${ }^{\mathrm{TM}}$ Kit (ThermoFisher Scientific Cat\# AM1907) to prevent genomic DNA contamination and cDNA was synthesized using the SuperScript ${ }^{\circledR}$ IV First-Strand Synthesis System (ThermoFisher Scientific Cat\#18091050). We performed RT qPCR with the BioRad CFX Connect Real-Time System (Cat \#1855201) to determine the relative expression of putative neuropeptide receptors across our tissue samples. Individual samples were prepared by combining prepared cDNA sample, [100um] forward and reverse primers, SsoFast EvaGreen Supermix (BioRad Cat \# 1725200) and nuclease free $\mathrm{diH}_{2} \mathrm{O}$ to a volume of $10 \mathrm{ul}$. $\mathrm{RT}^{-}$samples, no template controls (NTCs) and positive controls with Manduca genomic DNA from the brain were run for every plate. RT $^{-}$ and NTC had no amplification for all receptors and sample types run at $58.3^{\circ} \mathrm{C}$ (See Table 3 ). 
Optimal annealing temperatures were determined through a gradient test on genomic DNA to ensure qPCR on cDNA was performed at optimal temperature. All primer sets, including the reference gene, $\mathrm{RpS} 3$, were run using the following protocol $\left(95^{\circ} \mathrm{C} 2\right.$ minutes, $\left(95^{\circ} \mathrm{C} 5\right.$ seconds-> $58.3^{\circ} \mathrm{C} 30$ seconds) $x 39$ cycles, $65.0^{\circ} \mathrm{C} 5$ seconds stepped up to $95^{\circ} \mathrm{C}$ ) except for Mas-ATr primers, which were annealed at a temperature of $52^{\circ} \mathrm{C}$. All samples for $\mathrm{RpS} 3$ were run again at $52^{\circ} \mathrm{C}$ to ensure accurate calculation of relative expression values for Mas-ATr. Cq values for ANTa (antennae sample a), Mb (Medial cell cluster sample b) and NTC sample for the RpS3 run at $52^{\circ} \mathrm{C}$ were high (see Table 3). However, amplification curves revealed that there were no sharp amplification peaks and thus, high $\mathrm{Cq}$ values were due to noise not contamination. High Cq values with non-descript peaks for RpS3 NTCs run at $52^{\circ} \mathrm{C}$ were considered 0 for ANTa, Mb and NTC when calculating relative expression.

qPCR relative expression analysis: Raw qPCR data can be found in Table 3. Delta $\mathrm{Ct}\left(\mathrm{Ct}_{\text {receptor- }}\right.$

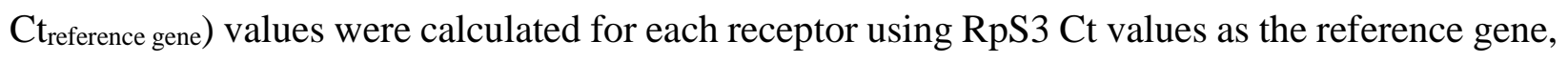
and averaged across all biological replicates for brain, lateral cell cluster, MCC and antennae tissue samples. Relative expression levels $\left(2^{-\Delta \mathrm{CT}}\right)$ were calculated for all receptors. $\mathrm{Ct}$ values less than or equal to 37 were considered non-detectable. All graphical representations for receptor qPCR were performed in GraphPad prism (v. 5.01).

Computational analysis of transmitter co-expression: We wrote a MATLAB (Naticks MA) program to determine if our observed transmitter co-expression data could be replicated by independent probabilities of expression of each transmitter. Given the known total number of LNs in the lateral cell cluster, and the total number of LNs expressing each neuropeptide from our cell counts, the model determines the probability of a given neuron co-expressing two transmitters. The program is given the average number of cells expressing a given neurotransmitter and then randomly assigns them to one of the cells in the cluster. The model can thus determine the probability of pairwise co-expression (i.e. 100\% of TK cells express MIP) in the lateral cell cluster based on chance. Specifically, using our observed data as the backbone of the model, we designed a matrix with 6 columns, 1 per transmitter type (TK, FMRF, Mas-AT, MIP, AST-A, GABA), with a row length of 360 long (the total number of LNs in the lateral cell cluster) (Homberg et al., 1988). Within each column, the model randomly distributes the number of cells that express a given 
transmitter to a row between 1 and 360 (Fig. 3A). For example, if we know that 12 cells within the lateral cell cluster are TKergic, the model randomly picks 12 numbers between 1 and 360 in the TK column, and marks that cell as TK positive. The number of cells expressing a certain neurotransmitter is chosen probabilistically based on the observed average and standard deviation of the number of neurons that express a given transmitter. With each iteration of the model, the cells that are assigned as 'transmitter positive' within each column are randomized. The model does this with all respective cell count totals for each transmitter column and then calculates the percentage each transmitter is expressed with another transmitter based on independent expression of each transmitter (across all pairwise comparisons). Standard deviation and percentages of coexpression were recorded across 10,000 iterations of the model. We then compared our observed co-expression percentages to the model's output to determine if independent probabilities of expression of each transmitter could explain observed co-expression.

The model described above has no initial assumption about the likelihood of co-expression and only the overall number of cells expressing each of the transmitter is determined initially. We used a similar model to determine if assigning dependent co-expression relationships for specific pairs of transmitters could replicate the co-expression patterns for other transmitter pairs. To do this, we built certain co-expression relationship explicitly as initial assumptions. For example, if we know that on average $100 \%$ of TKergic cells are also MIPergic, the program explicitly forces $100 \%$ of the cells that are assigned to be TK positive to also be MIP positive. This co-expression relationship is thus no longer determined based on independent expression probabilities like the first version of the script, but rather is an initial assumption - a rule. We can then determine if this rule alone shifts the co-expression of other transmitter types closer to the observed co-expression percentages. We applied these rules one by one (for a total of 92 different models), for every pairwise comparison of co-expression and statistically compared the output of the independent expression model to the output of the rule based model as well as the observed co-expression patterns we identified with immunocytochemistry. This allowed us to determine if specific coexpression relationships could replicate other co-expression relationships within LNs. The script was run on a Windows 7 desktop, with an Intel $\AA^{\circledR}$ Core ${ }^{\mathrm{TM}}$ 17-3770 CPU @ 3.4GHz processor, and a 64-bit operating system. 
Code Accessibility: Custom MatLab scripts available at [URL redacted for double-blind review], at [URL redacted for double-blind review], or upon request. The code is available as Extended Data.

Experimental Design and Statistical Analysis: The model outputs a predicted percentage of coexpression for every pairwise co-expression relationship. To statistically determine how well the model replicated observed co-expression $\%$ s, we used standard deviation indices (SDIs) to determine how close the model's predicted co-expression \% is to observed probability of coexpression. Similar to a Z-score, this measure is calculated as follows:

$\mathrm{SDI}=\left(\mathrm{Mean}_{\text {model }}-\mathrm{Mean}_{\text {observed }}\right) / \mathrm{stdev}_{\text {greatest }}$

Where Mean $_{\text {model }}=$ Mean probability of co-expression of any two given neurotransmitters from the model e.g. mean \% TK co-expressed with MIP

Mean $_{\text {observed }}=$ Mean probability of co-expression of any two given neurotransmitters from the observed co-expression relationships

stdev greatest $=$ the greatest standard deviation from either the model or observed data for a given coexpression relationship.

Weighted SDI's were calculated to reflect the match between data and model for the overall population of LN by weighting the contribution of each neurotransmitter proportionally to its prevalence.

Weighted SDI $=\sum\left(\left(\text { Mean }_{\text {model }}-\text { Mean }_{\text {observed }} / \mathrm{stdev}_{\text {greatest }}\right)^{*}\left(\mathrm{n}_{\text {co-expressed }} / \mathrm{n}_{\text {total }}\right)\right.$

For example, there are only 12 TK neurons in a total of 360 LNs, but 142 Mas-AT neurons. Therefore, predicting the number of Mas-AT neurons vs. TK neurons should carry more weight when determining the accuracy of each model. Weighted SDIs for each co-expression relationship (i.e. weighted SDI for the TK/MIP co-expression) were summed across relationships for an overall measure of the accuracy with which each model iteration replicated observed co-expression patterns.

SDI values can be interpreted by the following scale: 0: perfect consensus between model and experimental data; 1: model results are within one standard deviation of experimental data and thus replicate the data reasonably well; 2: model results are within two standard deviation of experimental data and thus do not replicate the data accurately. To determine the $\%$ improvement of each model at replicating observed co-expression (Fig 3G), all weighted SDI's were normalized with respect to the weighted SDI of the independent expression model using the formula: 
$\%$ improvement from "independent-expression" model $=\left(1\right.$-(weighted $\mathrm{SDI}_{\mathrm{x}} /$ weighted $\left.\mathrm{SDI}_{\text {ind }}\right) * 100$

All statistics were performed in GraphPad prism (v. 5.01).

\section{Results:}

The antennal lobe (AL) of Manduca is surrounded by 3 cell clusters that house the cell bodies of projection neurons and LNs. The lateral cell cluster consists of $\sim 950$ cell bodies, including 590 projection neurons and $\sim 360$ total LNs (Homberg et al., 1988), of which $\sim 170$ are GABAergic (Hoskins et al., 1986). Manduca LNs are diverse across several traits with no correlations between physiological properties, morphological properties, or GABA expression patterns in LNs (Reisenman et al., 2011). We therefore took a systematic approach to determine if transmitter co-expression could reliably sub-categorize and explain the apparent heterogeneity of LN cellular properties.

\section{Neuropeptide co-expression is highly heterogeneous}

We first determined the pairwise co-expression relationships for GABA and multiple neuropeptides Tachykinin (TK), FMRFamide (FMRF), Allatotropin (Mas-AT), Myoinhibitory peptide (MIP) and Allatostatin-A (AST-A) in LNs (Fig. 1A-E). We chose these neuropeptides because there are available antibodies of sufficient quality, we have performed the proper preadsorption controls for each of them, and finally these neuropeptides have the best functional, biochemical, and developmental characterization in Manduca as well as other insect species (Carroll et al., 1986; Blackburn et al., 2001; Skaer et al., 2002; Teal, 2002; Utz and Schachtner, 2005; Utz et al., 2007; Yapici et al., 2008; Ignell et al., 2009; Asahina et al., 2014; Ko et al., 2015). All moths were naïve and unmated adults, and equal number of males and females were used for each transmitter combination. Using a paired t-test we found no significant differences between the left and right lateral cell clusters for all peptides: Mas-AT ( $\mathrm{t}=1.718 ; \mathrm{df}=5 ; \mathrm{p}=0.1465)$, MIP $(t=0.1056 ; \mathrm{df}=5 ; \mathrm{p}=0.9200)$, FMRF $(\mathrm{t}=0.5324 ; \mathrm{df}=5 ; \mathrm{p}=0.6172)$, TK $(\mathrm{t}=1.085 ; \mathrm{df}=5 ; \mathrm{p}=0.3276)$, AST-A ( $\mathrm{t}=0.6407 ; \mathrm{df}=5 ; \mathrm{p}=0.5499)$. We also compared counts from male and female moths and using a paired t-test, we found no significant differences in cell counts between males and females for MIP $(\mathrm{t}=1.531 ; \mathrm{df}=2 ; \mathrm{p}=0.2654)$, AST-A $(\mathrm{t}=0.4187 ; \mathrm{df}=2 ; \mathrm{p}=0.7161), \mathrm{TK}(\mathrm{t}=0.0000 ; \mathrm{df}=2$; $\mathrm{p}=1.0), \mathrm{FMRF}(\mathrm{t}=0.1220 ; \mathrm{df}=2 ; \mathrm{p}=0.9141)$. There was a significant difference between males and 
females in Mas-AT expression ( $\mathrm{t}=11.97 ; \mathrm{df}=2 ; \mathrm{p}=0.0069$ ). Peptidergic LNs predominantly coexpressed GABA (Fig. 1F, Table 1), suggesting that LNs can be broadly subdivided into GABAergic/peptidergic and non-GABAergic/non-peptidergic LNs. The non-GABAergic LNs have the potential to be glutamatergic as RT-qPCR on lateral cell cluster mRNA revealed that the vesicular glutamate transporter (vGLUT) was highly expressed relative to a reference gene (40s ribosomal protein $\mathrm{s} 3 ; \mathrm{RpS} 3$, see Table 3 for Cq values). A large population of glutamatergic LNs in Manduca, in addition to the GABAergic LNs, would be consistent with the organization of the Drosophila AL (Das et al., 2011; Liu and Wilson, 2013). We then determined the co-expression ratios (i.e. what percentage of $\mathrm{X}$ expressing neurons co-express $\mathrm{Y}$ ) of every pairwise combination of TK, FMRF, Mas-AT and MIP (Fig. 2A-G). There were few consistent co-expression patterns, suggesting that most LNs co-express multiple neuropeptides to a variable degree (Fig. 2 E, G, C). The exception to this rule was TK which was co-expressed 100\% with MIP and never co-expressed with FMRF and Mas-AT (Fig. 2A, B, D, H). The 12 TKergic LNs were therefore the only LNs that expressed a consistent transmitter profile. Our results are consistent with other studies of GABA and peptide expression in Manduca (Hoskins et al., 1986; Homberg et al., 1990; Utz et al., 2008). Co-expression ratios for each pairwise co-expression relationship (i.e. $\%$ of neurons that co-express $\mathrm{X}$ and $\mathrm{Y}$ ) revealed that apart from TK, neuropeptides were co-expressed to a variable degree (Fig. 2H).

\section{Computational analysis of transmitter co-expression reveals that independent expression probability cannot explain observed transmitter co-expression in LNs}

Two possible scenarios can explain the lack of apparent systematic association between specific neuropeptides co-expressed by LNs. In one scenario, expression of a given neuropeptide is independent of the expression of another and the likelihood of specific co-expression patterns is equal to the independent probabilities of expression of each transmitter given the number of LNs that express each transmitter. Alternatively, specific pairs of neuropeptides are co-expressed more (or less) often than by chance, and a certain number of such relationships can explain the overall pattern of neuropeptide co-expression. To test these scenarios, we began by using computational modeling to test the hypothesis that co-expression could be explained independent probabilities of expression of each transmitter alone. Given the known total number of LNs in the lateral cell cluster (360;(Homberg et al., 1988) and the total number of LNs expressing each neuropeptide (Fig. 1), the model calculates the probability of a neuron co-expressing two transmitters (Fig. 3A; 
see methods). The model predicts the percentage of neurons that co-express every pairwise relationship of transmitters in our study. For example, based on the number of LNs that express Mas-AT and the number of LNs that express FMRFamide, and the total number of LNs in the AL, the model determines that $12 \%$ of Mas-AT neurons should co-express FMRF if the probability of expressing the former is independent of the probability of expressing the latter. However, based on our immunocytochemical data, we observed that $22 \%$ of Mas-AT neurons co-express FMRF (Fig $2 \mathrm{H}$ ). We then compared every predicted co-expression ratio from the model (which assumes independent probabilities of expression of each transmitter for each pairwise relationship) to the observed co-expression ratios (Fig. 2H) and determined how well the model replicates observed co-expression (Fig. 3B). If co-expression probabilities can be replicated by a model that assumes independent expression of each transmitter, then as a result, no organizing co-expression dependencies will be identified.

We found that most co-expression relationships were not replicated by a model assuming independent transmitter expression (Figure 4A; "independent expression" model). To statistically measure how well our model replicated observed co-expression patterns we then used a standard deviation index (SDI) for every predicted pairwise co-expression relationship vs. observed coexpression. An SDI score of 0 indicates that our simulation perfectly recapitulates observed coexpression patterns, whereas an SDI score above 1 indicates poor performance of the model. Each predicted co-expression ratio from the model was compared to the observed co-expression ratios and a SDI was calculated $\left(\mathrm{SDI}=\left(\mathrm{Mean}_{\text {model }}-\mathrm{Mean}_{\text {observed }}\right) / \mathrm{stdev}_{\text {greatest }}\right)$. SDI scores for every pairwise co-expression relationship were statistically weighted (see methods), such that coexpression relationships that included a larger proportion of the total LN population carried more weight. SDI scores revealed that while an independent-expression model could replicate some coexpression relationships (with a weighted SDI of 1.49), independent-expression alone does not accurately replicate the observed co-expression (Fig. 4B).

\section{A few specific co-expression constraints allow replication of overall co-expression patterns}

Since the independent expression of each transmitter did not replicate the overall probabilities of co-expression patterns, we next sought to identify which co-expression relationship must be adjusted to replicate the overall structure of co-expression. We implemented, in our model, rules according to which the probability of expression of a transmitter is dependent 
on the expression of another transmitter (Fig 5A), thereby explicitly setting the probability of coexpression to its observed value (Fig 2H). Therefore, the model contains a set number of coexpression relationships in the form of rules (for example, $42 \%$ of MIPergic LNs co-express MasAT as observed from our immunocytochemistry), while leaving the rest of the relationships to emerge through probabilistically-independent expression. We tested 94 different model iterations, each containing different combinations of 'co-expression rules' to determine which combination(s) of rules best replicated overall observed co-expression (Figure 5B). This allowed us to identify predictive co-expression relationships in an unbiased manner. As expected, the ability of the model to replicate observed co-expression patterns improved as more rules were added, as shown by weighted SDIs from all model iterations (Fig. 5B-D).

However, some combinations of rules outperformed others. We first constrained the total number of cells in the model to the total number of GABAergic LNs (164 cells instead of 360 total LNs), as presence of GABA is a reliable predictor of peptide expression observed in this study. This constraint outperformed the independent-expression model, had a weighted SDI of 0.94 and accurately replicated more co-expression patterns (Fig. 6A). This suggests that much of the diversity of neuropeptide co-expression can be constrained to the sub-population of GABAergic LNs in our study. All remaining model iterations were constrained to the total number of GABAergic LNs (see Figure 5B, filled in symbols indicate models where total number of LNs = 164 (with stdev) GABAergic neurons). Unexpectedly, one particular model that only contained one co-expression rule outperformed most models that were constrained by 2 and 3 rules (red square Fig. 5B). When the proportion of MIPergic LNs expressing Mas-AT is set to its observed value (42\%), the model replicated the highest number of co-expression relationships of all models with one rule (Fig. 6B-D), yielded the lowest weighted SDI (0.36), and outperformed the average of models with one rule (lower 95\% CI of mean: 0.6551, upper 95\% CI of mean: 0.9862), two rules (lower 95\% CI of mean: 0.5450, upper 95\% CI of mean: 0.7240), and even 3 rules (lower 95\% CI of mean: 0.4736 , upper 95\% CI of mean: 0.5897$)$. This was surprising because it suggested that replicating observed co-expression patterns did not require all co-expression relationships to be fixed, revealing specific proportional relationships that may be may be predictive of overall observed co-expression patterns. 
The only set of co-expression patterns that could not be replicated reasonably well in the model that included the GABA and the MIP/Mas-AT rules (as described above) involves TK. The 12 TK LNs (Lizbinski et al., 2016) follow a strict all-or-none neuropeptide co-expression pattern (100\% co-expression with MIP and 0\% co-express with Mas-AT or FMRFamide). Consistent with our data, TK LNs in the AL of the moth, Heliothis virescens also do not co-express FMRF or MasAT (Berg et al., 2007). This co-expression pattern cannot be replicated through independent expression models, even when several other rules are considered (Fig 6E). These co-expression patterns are so clear-cut that it set TK apart from other transmitters observed in this study.

\section{GABAв and neuropeptide receptors are expressed across all principal neuron types of the} AL

It may be unnecessary to tightly regulate co-expression of neuropeptides in specific subpopulations of LNs simply because specific classes of AL neurons express different sets of neuropeptide receptors. Thus, the heterogeneous transmitter profiles of individual LNs may not matter functionally because the impact of individual peptides within a modulatory cocktail of many peptides may be segregated due to neuron class-specific expression of each receptor. For instance, if olfactory receptor neurons (ORNs) express the MIP receptor and projection neurons (PNs) express the Mas-AT receptor, the influence of these two neuropeptides could differentially target input and output of the network, rather target the same neuron, resulting in different consequences on the network. However, this does not appear to be the case in this network, as we did not find differential expression of the receptors for the peptides examined in this study between ORNs, LNs and PNs. We first identified transcripts from the Manduca genome (Kanost et al., 2016) with high sequence identity to neuropeptide receptors identified from reference genomes in closely related species (Table 2). Then, using RT-qPCR, we determined the relative expression of five neuropeptide receptors (Mas-AT, MIP, AST-A, FMRF, TK), and the GABAB receptor in mRNA from the antennae (which house ORNs), the medial cell cluster (which houses only PNs), the lateral cell cluster (which houses LNs and PNs) and whole brains (as a positive control). Although the receptors for Mas-AT, MIP, AST-A, FMRF, GABAB, were detected in all four tissue types, the TK receptor was not detected in the lateral cell cluster (Fig. 7, for raw qPCR data see Table 3). This suggests that TKergic LNs differ from other LNs both in their co-expression patterns and their postsynaptic targets. Although we could not assess receptor expression on a neuron-by- 
neuron basis, our results suggest that a single LN releasing neuropeptides from at least four individual peptide families can have a powerful effect on the network, potentially affecting all three major cell classes in the AL.

\section{Discussion:}

Broad neuronal classes are surprisingly heterogeneous across many parameters and subclasses often exhibit partially overlapping traits including transmitter co-expression. Our goal was to determine organizing principles of LN heterogeneity. Our results suggest that neuropeptide coexpression in the AL is both heterogeneous and partially overlapping across the entire population rather than consistent within specific sub-populations of LNs (Fig. 8). Thus, peptidergic modulation cannot be considered within the context of single neuropeptides as activation of any given LN results in a dynamic cocktail of modulators that have the potential to influence every level of olfactory processing within the AL. Specifically, we find that transmitter profile is heterogeneous across LNs, with individual olfactory LNs capable of expressing the main inhibitory transmitter GABA and peptides from at least four families, and few instances in which transmitters are consistently co-expressed. Observed co-expression patterns cannot be explained by independent probabilities of expression of each transmitter (Fig. 4). Our analyses point to three organizing principles that once taken into consideration allow replication of overall co-expression structure: 1- peptidergic neurons are highly likely to co-express GABA; 2- the probability of expressing Mas-AT is dependent on MIP expression; 3- the all-or-none co-expression patterns of TKergic neurons with several other neuropeptides (MIP, FMRF and Mas-AT). For other pairs, the presence of one transmitter was not predictive of the presence of the other and thus co-expression probability could be replicated by independent probabilities. The stochastic nature of these coexpression patterns argues against clear-cut, exclusive definition of subpopulations based on the presence of single neuropeptides. Furthermore, the receptors for GABA and all neuropeptides in this study were expressed within each population of principal neuron type in the AL (Fig. 7) suggesting that peptides released from LNs potentially influence every level of olfactory processing within the AL. Overall, we demonstrate that peptide expression is partially overlapping across LNs and thus sub-populations of LNs cannot be functionally defined based on the presence of single peptides. Furthermore, neuropeptide and $\mathrm{GABA}_{\mathrm{B}}$ receptors were expressed within each population of principal neuron type in the AL because ORNs, the influence of peptides are not 
segregated based on cell class-specific receptor expression. Thus, co-release of peptides and GABA likely mediate a complex mix modulation to control the dynamic range of the AL, providing multiple mechanisms to alter olfactory processing.

Heterogeneous transmitter co-expression is a common theme within GABAergic LNs across vertebrates and invertebrates alike (Homberg et al., 1990; Maccaferri and Lacaille, 2003; Flames and Marin, 2005; Utz et al., 2008; Carlsson et al., 2010; DeFelipe et al., 2013; Siju et al., 2013; Binzer et al., 2014; Gabitto et al., 2016; Yavorska and Wehr, 2016; Diesner et al., 2018b). MALDI-TOF spectrometry revealed that at least 12 known peptides are expressed in developing Manduca ALs (Utz et al., 2007), suggesting that co-expression patterns are likely even more complex than detailed here. Furthermore, the antibodies used in this study recognize multiple isoforms of peptides within the same family (i.e. FMRF has multiple isoforms), and thus there are almost certainly more organizational principals underlying heterogeneous peptide expression than discussed here. Other insects including mosquitos (Siju et al., 2013), other species of moths (Berg et al., 2007; Diesner et al., 2018b), beetles (Binzer et al., 2014) and fruit flies (Carlsson et al., 2010; Hussain et al., 2016; Croset et al., 2018), express a large number of peptides within their olfactory systems suggesting that peptides likely play an important yet functionally underexplored role in shaping olfactory responses. One exception to the theme of heterogeneous co-expression was that the TK neurons differed in their patterns of co-expression from other peptidergic LNs. All TK LNs co-expressed MIP, and none co-expressed FMRF or Mas-AT, suggesting that TK LNs are primarily inhibitory as TK and MIP receptors are inhibitory in Drosophila (Yapici et al., 2008; Ignell et al., 2009; Ko et al., 2015). Furthermore, TK receptor transcripts were not detected in lateral cell cluster mRNA and thus not in LNs, although TK/MIPergic LNs could still influence LNs via GABA $\mathrm{B}$ and MIP receptor. In Drosophila melanogaster, TK mediates presynaptic gain control upon ORNs (Ignell et al., 2009), and TKr expression in Manduca ORNs is consistent with this finding. This suggests that TK LNs may play a distinct role from other LNs in olfactory processing which could include presynaptic gain control.

Very few non-GABAergic LNs co-express the neuropeptides we examined here, however they are still a sizeable proportion of the total number of LNs and likely as heterogeneous as GABAergic LNs. We did not definitively identify the transmitter released by these LNs, however we did detect the expression of vGlut mRNA within the lateral cell cluster (Fig. 7), making 
glutamate a candidate transmitter for the non-GABAergic LNs. Similar to GABAergic LNs, glutamatergic LNs in Drosophila are particularly diverse in their morphology (Das et al., 2011) but appear to differ from GABAergic LNs in their synaptic targets by predominantly affecting PNs (Liu and Wilson, 2013), while GABAergic LNs in Drosophila affect ORNs, LNs and PNs (Wilson and Laurent, 2005; Olsen and Wilson, 2008; Root et al., 2008; Hong and Wilson, 2015). Future studies should confirm whether the non-GABAergic population observed here are truly glutamatergic.

The probability of expression of certain transmitters appear to be dependent on one another. In particular, we showed that the probability of expressing Mas-AT is dependent on the expression of MIP (Figure 6). While the goal of our study is not to determine the developmental mechanisms that underlie co-expression, it is important to note that developmental mechanisms of peptidergic regulation most certainly shape observed heterogeneous co-expression. For instance, the molting hormone 20-hydroxyecdysone induces Mas-AT expression in LNs and other neuropeptides in the AL of Manduca (Utz and Schachtner, 2005; Utz et al., 2007), implying that co-expression patterns may reflect extrinsic developmental cues that guide the development of specific peptide expressing LNs. Furthermore, both Mas-AT and MIP expressing LNs arise slightly before and during the formation of glomeruli, suggesting that temporal expression patterns of these peptides may play a role in the development of AL structure and function (Utz et al., 2007). Interestingly, the model constraint best able to replicate observed co-expression across all LNs in our study was the proportional relationship between MIP/Mas-AT expressing neurons.

However, the developmental mechanisms that control peptide expression in LNs of Manduca are unknown. In Drosophila, the transcription factor DIMMED targets many genes involved in peptide expression (Hewes et al., 2003; Gauthier and Hewes, 2006; Hewes et al., 2006; Park et al., 2008b; Park and Taghert, 2009; Hadzic et al., 2015) and dense core vesicle production (Hamanaka et al., 2010; Park et al., 2014). DIMMED likely acts in a combinatorial manner with other cell-specific transcription factors to determine peptide expression in individual neurons (Liu et al., 2016; Stratmann and Thor, 2017). Although DIMMED doesn't target any single neuropeptide gene (Hadzic et al., 2015) other transcription factors do regulate sub-type specific neuropeptide expression (Allan et al., 2003; Berndt et al., 2015). While DIMMED positive neurons co-express multiple peptides, not all peptidergic neurons express DIMMED (Park et al., 2008a; 
Diesner et al., 2018a), and the role DIMMED of in Manduca has not been established. Regardless, a similar combinatorial transcriptional code could underlie the heterogeneity of peptide expression observed here. Furthermore, in cortex, LN subtypes arise from unique progenitors (Anderson et al., 1997; Wichterle et al., 2001; Nery et al., 2002; Kepecs and Fishell, 2014) and their diversity is shaped by additional factors (Flames and Marin, 2005) including neural activity (Patz et al., 2004; De Marco Garcia et al., 2011) transcription factor expression (Mayer et al., 2018; Sweeney et al., 2018) and growth factors (Huang et al., 1999). Similarly, GABAergic and glutamatergic LNs in Drosophila arise from distinct neuroblasts (Das et al., 2008; Das et al., 2011), and glomerular innervation patterns of LNs require ORN axons during development (Chou et al., 2010), suggesting that heterogeneity of LNs may be due in part to distinct origins and/or activity of other neurons in the network.

Our study reveals some expression co-dependencies, but also highlights the apparent stochastic nature of other co-expression patterns. There are several examples of biological systems in which features like gene expression in E. coli clones (Elowitz et al., 2002; Raj and van Oudenaarden, 2008; Huh and Paulsson, 2011), behavior (Honegger and de Bivort, 2018), or anatomical layout (Caron et al., 2013), appear to be randomly structured or stochastic. For example, random combinations of AL PNs from different glomeruli converge and synapse upon individual mushroom body Kenyon cells in Drosophila regardless of anatomy, developmental origin or odor tuning, thus abandoning the odor-topic organization of the AL (Caron et al., 2013). Due to the stochastic heterogeneity of some transmitter co-expression patterns, our results suggest the presence of single peptides should not be used to functionally define classes of neurons. Additionally, this stochasticity suggests that LNs may not functionally require fixed compliments of transmitters.

We found that a single neuropeptide has the potential to simultaneously target every principal neuron type as all neuropeptide receptors were expressed by populations of ORNs, LNs and PNs. This network wide convergence of peptidergic modulation demonstrates that individual LNs do not differentially target principal neuron type based on differences in post-synaptic receptor expression. This further supports the idea that LN activation may serve to regulate multiple processing stages within the olfactory network by simultaneously targeting AL input, output and local processing. However, individual neurons within each principal AL neuron type 
may exhibit differential receptor expression, as we were not able to assess receptor expression at the level of individual neurons. Future studies should determine if neuropeptide receptor expression is as heterogeneous as neuropeptide co-expression itself as there are likely subpopulations of neurons that exhibit differential receptor expression. This may be further complicated as neuropeptide receptor expression can be regulated by physiological state, as observed for the role of hunger (Ko et al., 2015; Min et al., 2016) or mating state in Drosophila (Hussain et al., 2016). Peptide expression itself may be similarly regulated, as observed in feeding state of Aedes aegypti (Christ et al., 2017) or mating state of Agrotis ipsilon moths (Diesner et al., 2018b). All moths in our study were naïve and unmated, however this does not rule out the potential for physiological state to affect peptide expression in the AL.

Activation of even a single LN can mediate a complex mix of inhibition and/or excitation that varies in time course and strength due to the co-release of the small-transmitter GABA and a heterogeneous mix of peptides. LNs, apart from TK LNs, co-expressed multiple peptide families and GABA that activate both inhibitory (TK, sNPF, and sex-peptide/MIP) (Yapici et al., 2008; Ignell et al., 2009; Asahina et al., 2014; Ko et al., 2015) and excitatory receptors (Mas-AT and FMRF) (Horodyski et al., 2011; Lenz et al., 2015; Ormerod et al., 2015) via a mix of ionotropic and metabotropic signaling. Furthermore, AL neurons express both the GABAa and GABA receptors, and the effects $\mathrm{GABA}_{\mathrm{B}}$ receptor activation are far shorter-lasting relative to neuropeptide receptors (Salio et al., 2006). Thus, small-transmitter and peptide co-expression expands the temporal scale with which a single neuron can alter network processing. However, it is unclear whether LNs employ bulk and/or restricted synaptic release of peptides, making the spatial scale of their influence unknown. Finally, the network may need to be more strongly activated (i.e. by higher concentrations of odors or increased length of odor-stimuli) for LNs to release neuropeptides due to the different calcium binding affinities of distinct synaptotagmins associated with small clear vesicles and dense-core vesicles (Saraswati et al., 2007; Li et al., 2009). Thus, the consequences of LN activation and peptidergic modulation may depend more upon the degree of network activity rather than the identity of any singular LN that is activated. Overall, this heterogeneous cocktail of peptides likely provides the AL with flexible options to up or down regulate olfactory processing over a variety of time frames and spatial scales within the context of ongoing network activity. 
Within the AL, combined GABAergic and peptide release from LNs could potentially play a variety of functional roles including autoinhibition, lateral excitation or inhibition, disinhibition, and even odor-specific processing. For example, lateral input from LNs scales with overall network activity as a means to control the dynamic range of the network and avoid response saturation of PNs (Olsen and Wilson, 2008; Root et al., 2008). Additionally, some glomeruli are more subject to inhibition than others simply due to differences in glomerulus-specific, nonuniform LN innervation (Wilson and Laurent, 2005; Chou et al., 2010) and ORN GABAb receptor expression (Root et al., 2008). As a result, the processing of specific odors differs in the degree of insulation from ongoing activity in the olfactory system, and specific glomeruli are therefore more (or less) insulated from presynaptic gain control mediated by both GABA (Root et al., 2008) and, potentially, neuropeptides (Ignell et al., 2009; Ko et al., 2015; Hussain et al., 2016). Spatial activation of Drosophila LNs is also odor-specific and heterogeneous, with LNs responding to either single, or multiple odors (Ng et al., 2002). The non-uniform innervation and heterogeneous odor-evoked responses of LNs suggests that the activation of LNs is a complex combinatorial process resulting in glomerular specific local processing. In Manduca, most GABAergic LNs are wide-field and heavily ramify all glomeruli, suggesting that the consequences of GABAergic LN activation cannot be fully segregated based on odor identity. However, a small sub-set of GABAergic and non-GABAergic LNs exhibit restricted glomerular arborizations, only innervating a small sub-section of the AL (Reisenman et al., 2011). Consequently, activation of morphologically restricted LNs may dis-inhibit or inhibit other LNs from neighboring glomeruli in an odor-specific manner in order to increase or decrease odor salience by altering the output of PNs (Hildebrand et al., 1992; Christensen et al., 1993). While no correlations between morphology (wide-field vs. restricted), physiology, odor-response profile, and transmitter content have been identified in Manduca LNs (Reisenman et al., 2011), it could be that wide-field vs. restricted LNs exhibit distinct and predictable combinations of peptides. These potential network consequences are likely applicable across insect species as LN heterogeneity is a recurring theme. Using physiology paired with hierarchical clustering based on morphology, multiple Drosophila LN subtypes exhibit broad correlations between morphology, physiology and genetic classes (Chou et al., 2010). However, LNs within the "patchy" cell type exhibit highly variable innervation patterns and considerable diversity exists even within other LN sub-types (Chou et al., 2010). Additionally, morphologically and functionally distinct classes of LNs exist in honeybees (Schafer and Bicker, 
1986; Flanagan and Mercer, 1989; Fonta et al., 1993; Sun et al., 1993; Bornhauser and Meyer, 1997; Seidel and Bicker, 1997; Galizia and Kimmerle, 2004; Dacks et al., 2010) and cockroaches (Malun, 1991; Distler and Boeckh, 1997; Loesel and Homberg, 1999; Husch et al., 2009a, b; Fusca et al., 2013; Fusca et al., 2015; Neupert et al., 2018). Ultimately, determining the roles of individual peptides will be challenging as complex patterns of co-expression must be integrated with knowledge of functionally distinct subtypes of LNs.

Reconciling within cell-type heterogeneity represents an ongoing challenge. Similar to LNs across taxa and brain region, Manduca LNs are highly heterogeneous across many parameters. This heterogeneity provides multiple coding strategies and mechanisms to neurons within the same population, expanding the role single neurons play in altering network function. The link between heterogeneous response properties and neural coding has been studied in a wide range of systems (Chelaru and Dragoi, 2008; Marsat and Maler, 2010; Ogawa et al., 2011; Pitkow and Meister, 2012; Ahn et al., 2014), however, the systematic analysis of heterogeneous traits like transmitter co-expression has not been as extensively explored. Here, we show that traits, like transmitter co-expression are partially overlapping across the entire LN population. Ultimately, our results demonstrate that peptidergic modulation cannot be considered within the context of single neuropeptides as activation of any given LN results in a dynamic cocktail of modulators that have the potential to influence every level of olfactory processing within the AL.

Author Contributions: All authors had full access to all the data in the study and take responsibility for the integrity of the data and the accuracy of the data analysis. Study concept and design: Kristyn M. Lizbinski (KML), Gary Marsat (GM), Andrew M. Dacks (AMD). Acquisition of data: KML Computational model: KML, GM. Analysis and interpretation of data: KML, GM, AMD. Drafting of the manuscript: KML, GM, AMD. Critical revision of the manuscript for important intellectual content: KML, GM, AMD. Obtained funding: AMD Administrative, technical, and material support: AMD. Study supervision: AMD

Acknowledgments: Acknowledgments: We thank Dr. Tim Driscoll and Dr. Tori Verhoeve for theiradvice and help with RT-qPCR experimental design and setup, Andrew Steele and Lillian Bailey for their help with cell counts, and Aditya Kesari for assistance with preliminary data as well as the other members of the Dacks lab for their support. We also thank Kate Allen, Tyler Sizemore, Philip Chapman, Dr. Kevin Daly, Dr. Sadie Bergeron, Dr. Quentin Gaudry, and Dr. 
Mani Ramaswami for helpful comments on this manuscript. The TK, AST-A, and Mas-AT

antibodies were provided by Dr. Jan Veenstra, the MIP antibody was provided by Dr. Christian Wegener and developed by Dr. Manfred Eckert, and the FMRF antibody by Dr. Eve Marder

\section{Works Cited}

Ache BW, Young JM (2005) Olfaction: diverse species, conserved principles. Neuron 48:417-430.

Ahn J, Kreeger LJ, Lubejko ST, Butts DA, MacLeod KM (2014) Heterogeneity of intrinsic biophysical properties among cochlear nucleus neurons improves the population coding of temporal information. Journal of neurophysiology 111:2320-2331.

Allan DW, St Pierre SE, Miguel-Aliaga I, Thor S (2003) Specification of neuropeptide cell identity by the integration of retrograde BMP signaling and a combinatorial transcription factor code. Cell 113:73-86.

Anderson SA, Eisenstat DD, Shi L, Rubenstein JL (1997) Interneuron migration from basal forebrain to neocortex: dependence on Dlx genes. Science 278:474-476.

Asahina K, Watanabe K, Duistermars BJ, Hoopfer E, Gonzalez CR, Eyjolfsdottir EA, Perona P, Anderson DJ (2014) Tachykinin-expressing neurons control male-specific aggressive arousal in Drosophila. Cell 156:221-235.

Bell RA, Joachim FG (1976) Techniques for Rearing Laboratory Colonies of Tobacco Hornworms and Pink Bollworms Lepidoptera-Sphingidae-Gelechiidae. Annals of the Entomological Society of America 69:365-373.

Berg BG, Schachtner J, Utz S, Homberg U (2007) Distribution of neuropeptides in the primary olfactory center of the heliothine moth Heliothis virescens. Cell Tissue Res 327:385-398.

Berndt AJ, Tang JC, Ridyard MS, Lian T, Keatings K, Allan DW (2015) Gene Regulatory Mechanisms Underlying the Spatial and Temporal Regulation of Target-Dependent Gene Expression in Drosophila Neurons. PLoS genetics 11:e1005754.

Binzer M, Heuer CM, Kollmann M, Kahnt J, Hauser F, Grimmelikhuijzen CJ, Schachtner J (2014) Neuropeptidome of Tribolium castaneum antennal lobes and mushroom bodies. The Journal of comparative neurology 522:337-357.

Blackburn MB, Jaffe H, Kochansky J, Raina AK (2001) Identification of four additional myoinhibitory peptides (MIPs) from the ventral nerve cord of Manduca sexta. Archives of insect biochemistry and physiology 48:121-128.

Bornhauser BC, Meyer EP (1997) Histamine-like immunoreactivity in the visual system and brain of an orthopteran and a hymenopteran insect. Cell Tissue Res 287:211-221.

Bradley SP, Chapman PD, Lizbinski KM, Daly KC, Dacks AM (2016) A Flight Sensory-Motor to Olfactory Processing Circuit in the Moth Manduca sexta. Frontiers in neural circuits 10:5.

Carlsson MA, Diesner M, Schachtner J, Nässel DR (2010) Multiple neuropeptides in the Drosophila antennal lobe suggest complex modulatory circuits. The Journal of comparative neurology 518:3359-3380.

Caron SJ, Ruta V, Abbott LF, Axel R (2013) Random convergence of olfactory inputs in the Drosophila mushroom body. Nature 497:113-117.

Carroll LS, Carrow GM, Calabrese RL (1986) Localization and release of FMRFamide-like immunoreactivity in the cerebral neuroendocrine system of Manduca sexta. The Journal of experimental biology 126:1-14.

Chelaru MI, Dragoi V (2008) Efficient coding in heterogeneous neuronal populations. Proceedings of the National Academy of Sciences of the United States of America 105:16344-16349. 
Chou YH, Spletter ML, Yaksi E, Leong JC, Wilson RI, Luo L (2010) Diversity and wiring variability of olfactory local interneurons in the Drosophila antennal lobe. Nature neuroscience 13:439-449.

Christ P, Reifenrath A, Kahnt J, Hauser F, Hill SR, Schachtner J, Ignell R (2017) Feeding-induced changes in allatostatin-A and short neuropeptide $\mathrm{F}$ in the antennal lobes affect odor-mediated host seeking in the yellow fever mosquito, Aedes aegypti. PloS one 12:e0188243.

Christensen TA, Hildebrand JG (1987) Male-specific, sex pheromone-selective projection neurons in the antennal lobes of the moth Manduca sexta. J Comp Physiol A 160:553-569.

Christensen TA, Waldrop BR, Harrow ID, Hildebrand JG (1993) Local interneurons and information processing in the olfactory glomeruli of the moth Manduca sexta. J Comp Physiol A 173:385399.

Cohen JY, Amoroso MW, Uchida N (2015) Serotonergic neurons signal reward and punishment on multiple timescales. eLife 4.

Croset V, Treiber CD, Waddell S (2018) Cellular diversity in the Drosophila midbrain revealed by singlecell transcriptomics. eLife 7.

Dacks AM, Reisenman CE, Paulk AC, Nighorn AJ (2010) Histamine-immunoreactive local neurons in the antennal lobes of the hymenoptera. The Journal of comparative neurology 518:2917-2933.

Daly KC, Kalwar F, Hatfield M, Staudacher E, Bradley SP (2013) Odor detection in Manduca sexta is optimized when odor stimuli are pulsed at a frequency matching the wing beat during flight. PloS one 8:e81863.

Das A, Sen S, Lichtneckert R, Okada R, Ito K, Rodrigues V, Reichert H (2008) Drosophila olfactory local interneurons and projection neurons derive from a common neuroblast lineage specified by the empty spiracles gene. Neural Dev 3:33.

Das A, Chiang A, Davla S, Priya R, Reichert H, Vijayraghavan K, Rodrigues V (2011) Identification and analysis of a glutamatergic local interneuron lineage in the adult Drosophila olfactory system. Neural Syst Circuits 1:4.

De Marco Garcia NV, Karayannis T, Fishell G (2011) Neuronal activity is required for the development of specific cortical interneuron subtypes. Nature 472:351-355.

DeFelipe J et al. (2013) New insights into the classification and nomenclature of cortical GABAergic interneurons. Nature reviews Neuroscience 14:202-216.

Diesner M, Predel R, Neupert S (2018a) Neuropeptide Mapping of Dimmed Cells of Adult Drosophila Brain. Journal of the American Society for Mass Spectrometry.

Diesner M, Gallot A, Binz H, Gaertner C, Vitecek S, Kahnt J, Schachtner J, Jacquin-Joly E, Gadenne C (2018b) Mating-Induced Differential Peptidomics of Neuropeptides and Protein Hormones in Agrotis ipsilon Moths. Journal of proteome research.

Distler PG, Boeckh J (1997) Synaptic connections between identified neuron types in the antennal lobe glomeruli of the cockroach, Periplaneta americana: II. Local multiglomerular interneurons. The Journal of comparative neurology 383:529-540.

Eddine R, Valverde S, Tolu S, Dautan D, Hay A, Morel C, Cui Y, Lambolez B, Venance L, Marti F, Faure $\mathrm{P}$ (2015) A concurrent excitation and inhibition of dopaminergic subpopulations in response to nicotine. Scientific reports 5:8184.

Elowitz MB, Levine AJ, Siggia ED, Swain PS (2002) Stochastic gene expression in a single cell. Science 297:1183-1186.

Flames N, Marin O (2005) Developmental mechanisms underlying the generation of cortical interneuron diversity. Neuron 46:377-381.

Flanagan D, Mercer A (1989) Morphology and response characteristics of neurones in the deutocerebrum of the brain in the honeybeeApis mellifera. Journal of comparative physiology A, Neuroethology, sensory, neural, and behavioral physiology 164:483-494.

Fonta C, Sun X, Masson C (1993) Morphology and spatial distribution of bee antennal lobe interneurones responsive to odours. Chemical senses 18:101-119.

Fusca D, Schachtner J, Kloppenburg P (2015) Colocalization of allatotropin and tachykinin-related peptides with classical transmitters in physiologically distinct subtypes of olfactory local 
interneurons in the cockroach (Periplaneta americana). The Journal of comparative neurology 523:1569-1586.

Fusca D, Husch A, Baumann A, Kloppenburg P (2013) Choline acetyltransferase-like immunoreactivity in a physiologically distinct subtype of olfactory nonspiking local interneurons in the cockroach (periplaneta americana). The Journal of comparative neurology 521:3556-3569.

Gabitto MI, Pakman A, Bikoff JB, Abbott LF, Jessell TM, Paninski L (2016) Bayesian Sparse Regression Analysis Documents the Diversity of Spinal Inhibitory Interneurons. Cell 165:220-233.

Galizia CG, Kimmerle B (2004) Physiological and morphological characterization of honeybee olfactory neurons combining electrophysiology, calcium imaging and confocal microscopy. Journal of comparative physiology A, Neuroethology, sensory, neural, and behavioral physiology 190:2138.

Gauthier SA, Hewes RS (2006) Transcriptional regulation of neuropeptide and peptide hormone expression by the Drosophila dimmed and cryptocephal genes. The Journal of experimental biology 209:1803-1815.

Gonchar Y, Wang Q, Burkhalter A (2007) Multiple distinct subtypes of GABAergic neurons in mouse visual cortex identified by triple immunostaining. Frontiers in neuroanatomy 1:3.

Hadzic T, Park D, Abruzzi KC, Yang L, Trigg JS, Rohs R, Rosbash M, Taghert PH (2015) Genome-wide features of neuroendocrine regulation in Drosophila by the basic helix-loop-helix transcription factor DIMMED. Nucleic acids research 43:2199-2215.

Hamanaka Y, Park D, Yin P, Annangudi SP, Edwards TN, Sweedler J, Meinertzhagen IA, Taghert PH (2010) Transcriptional orchestration of the regulated secretory pathway in neurons by the bHLH protein DIMM. Current biology : CB 20:9-18.

Hewes RS, Park D, Gauthier SA, Schaefer AM, Taghert PH (2003) The bHLH protein Dimmed controls neuroendocrine cell differentiation in Drosophila. Development 130:1771-1781.

Hewes RS, Gu T, Brewster JA, Qu C, Zhao T (2006) Regulation of secretory protein expression in mature cells by DIMM, a basic helix-loop-helix neuroendocrine differentiation factor. The Journal of neuroscience : the official journal of the Society for Neuroscience 26:7860-7869.

Hildebrand JG, Christensen TA, Harrow ID, Homberg U, Matsumoto SG, Waldrop BR (1992) The roles of local interneurons in the processing of olfactory information in the antennal lobes of the moth Manduca sexta. Acta Biol Hung 43:167-174.

Homberg U, Montague RA, Hildebrand JG (1988) Anatomy of antenno-cerebral pathways in the brain of the sphinx moth Manduca sexta. Cell Tissue Res 254:255-281.

Homberg U, Kingan TG, Hildebrand JG (1990) Distribution of FMRFamide-like immunoreactivity in the brain and suboesophageal ganglion of the sphinx moth Manduca sexta and colocalization with SCPB-, BPP-, and GABA-like immunoreactivity. Cell Tissue Res 259:401-419.

Honegger K, de Bivort B (2018) Stochasticity, individuality and behavior. Current biology : CB 28:R8R12.

Hong EJ, Wilson RI (2015) Simultaneous encoding of odors by channels with diverse sensitivity to inhibition. Neuron 85:573-589.

Horodyski FM, Verlinden H, Filkin N, Vandersmissen HP, Fleury C, Reynolds SE, Kai ZP, Broeck JV (2011) Isolation and functional characterization of an allatotropin receptor from Manduca sexta. Insect Biochem Mol Biol 41:804-814.

Hoskins SG, Homberg U, Kingan TG, Christensen TA, Hildebrand JG (1986) Immunocytochemistry of GABA in the antennal lobes of the sphinx moth Manduca sexta. Cell Tissue Res 244:243-252.

Huang ZJ, Kirkwood A, Pizzorusso T, Porciatti V, Morales B, Bear MF, Maffei L, Tonegawa S (1999) BDNF regulates the maturation of inhibition and the critical period of plasticity in mouse visual cortex. Cell 98:739-755.

Huh D, Paulsson J (2011) Non-genetic heterogeneity from stochastic partitioning at cell division. Nature genetics 43:95-100. 
Husch A, Paehler M, Fusca D, Paeger L, Kloppenburg P (2009a) Calcium current diversity in physiologically different local interneuron types of the antennal lobe. The Journal of neuroscience : the official journal of the Society for Neuroscience 29:716-726.

Husch A, Paehler M, Fusca D, Paeger L, Kloppenburg P (2009b) Distinct electrophysiological properties in subtypes of nonspiking olfactory local interneurons correlate with their cell type-specific $\mathrm{Ca} 2+$ current profiles. Journal of neurophysiology 102:2834-2845.

Hussain A, Ucpunar HK, Zhang M, Loschek LF, Grunwald Kadow IC (2016) Neuropeptides Modulate Female Chemosensory Processing upon Mating in Drosophila. PLoS biology 14:e1002455.

Ignell R, Root CM, Birse RT, Wang JW, Nässel DR, Winther AM (2009) Presynaptic peptidergic modulation of olfactory receptor neurons in Drosophila. Proceedings of the National Academy of Sciences of the United States of America 106:13070-13075.

Jiang H, Wang Y, Kanost MR (1996) Primary structure of ribosomal proteins S3 and S7 from Manduca sexta. Insect Mol Biol 5:31-38.

Kanost MR et al. (2016) Multifaceted biological insights from a draft genome sequence of the tobacco hornworm moth, Manduca sexta. Insect Biochem Mol Biol 76:118-147.

Kepecs A, Fishell G (2014) Interneuron cell types are fit to function. Nature 505:318-326.

Ko KI, Root CM, Lindsay SA, Zaninovich OA, Shepherd AK, Wasserman SA, Kim SM, Wang JW (2015) Starvation promotes concerted modulation of appetitive olfactory behavior via parallel neuromodulatory circuits. eLife 4.

Lenz O, Xiong J, Nelson MD, Raizen DM, Williams JA (2015) FMRFamide signaling promotes stressinduced sleep in Drosophila. Brain Behav Immun 47:141-148.

Li H, Horns F, Wu B, Xie Q, Li J, Li T, Luginbuhl DJ, Quake SR, Luo L (2017) Classifying Drosophila Olfactory Projection Neuron Subtypes by Single-Cell RNA Sequencing. Cell 171:1206-1220 e1222.

Li J, Xiao Y, Zhou W, Wu Z, Zhang R, Xu T (2009) Silence of Synaptotagmin VII inhibits release of dense core vesicles in PC12 cells. Science in China Series C, Life sciences 52:1156-1163.

Liang X, Holy TE, Taghert PH (2017) A Series of Suppressive Signals within the Drosophila Circadian Neural Circuit Generates Sequential Daily Outputs. Neuron 94:1173-1189 e1174.

Liu WW, Wilson RI (2013) Glutamate is an inhibitory neurotransmitter in the Drosophila olfactory system. Proceedings of the National Academy of Sciences of the United States of America 110:10294-10299.

Liu Y, Luo J, Nässel DR (2016) The Drosophila Transcription Factor Dimmed Affects Neuronal Growth and Differentiation in Multiple Ways Depending on Neuron Type and Developmental Stage. Frontiers in molecular neuroscience 9:97.

Lizbinski KM, Metheny JD, Bradley SP, Kesari A, Dacks AM (2016) The anatomical basis for modulatory convergence in the antennal lobe of Manduca sexta. The Journal of comparative neurology 524:1859-1875.

Loesel R, Homberg U (1999) Histamine-immunoreactive neurons in the brain of the cockroach Leucophaea maderae. Brain research 842:408-418.

Maccaferri G, Lacaille JC (2003) Interneuron Diversity series: Hippocampal interneuron classifications-making things as simple as possible, not simpler. Trends in neurosciences 26:564-571.

Malun D (1991) Inventory and distribution of synapses of identified uniglomerular projection neurons in the antennal lobe of Periplaneta americana. The Journal of comparative neurology 305:348-360.

Marder E, Calabrese RL, Nusbaum MP, Trimmer B (1987) Distribution and partial characterization of FMRFamide-like peptides in the stomatogastric nervous systems of the rock crab, Cancer borealis, and the spiny lobster, Panulirus interruptus. The Journal of comparative neurology 259:150-163.

Marsat G, Maler L (2010) Neural heterogeneity and efficient population codes for communication signals. Journal of neurophysiology 104:2543-2555.

Mayer C, Hafemeister C, Bandler RC, Machold R, Brito RB, Jaglin X, Allaway K, Butler A, Fishell G, Satija R (2018) Developmental diversification of cortical inhibitory interneurons. Nature. 
Min S, Chae HS, Jang YH, Choi S, Lee S, Jeong YT, Jones WD, Moon SJ, Kim YJ, Chung J (2016) Identification of a Peptidergic Pathway Critical to Satiety Responses in Drosophila. Current biology : CB 26:814-820.

Nässel DR (2018) Substrates for Neuronal Cotransmission With Neuropeptides and Small Molecule Neurotransmitters in Drosophila. Frontiers in cellular neuroscience 12:83.

Nery S, Fishell G, Corbin JG (2002) The caudal ganglionic eminence is a source of distinct cortical and subcortical cell populations. Nature neuroscience 5:1279-1287.

Neupert S, Fusca D, Kloppenburg P, Predel R (2018) Analysis of Single Neurons by Perforated Patch Clamp Recordings and MALDI-TOF Mass Spectrometry. ACS chemical neuroscience 9:20892096.

Ng M, Roorda RD, Lima SQ, Zemelman BV, Morcillo P, Miesenbock G (2002) Transmission of olfactory information between three populations of neurons in the antennal lobe of the fly. Neuron 36:463-474.

Nusbaum MP, Blitz DM, Marder E (2017) Functional consequences of neuropeptide and small-molecule co-transmission. Nature reviews Neuroscience 18:389-403.

Nusbaum MP, Blitz DM, Swensen AM, Wood D, Marder E (2001) The roles of co-transmission in neural network modulation. Trends in neurosciences 24:146-154.

Ogawa T, Riera J, Goto T, Sumiyoshi A, Nonaka H, Jerbi K, Bertrand O, Kawashima R (2011) Largescale heterogeneous representation of sound attributes in rat primary auditory cortex: from unit activity to population dynamics. The Journal of neuroscience : the official journal of the Society for Neuroscience 31:14639-14653.

Okaty BW, Freret ME, Rood BD, Brust RD, Hennessy ML, deBairos D, Kim JC, Cook MN, Dymecki SM (2015) Multi-Scale Molecular Deconstruction of the Serotonin Neuron System. Neuron 88:774-791.

Olsen SR, Wilson RI (2008) Lateral presynaptic inhibition mediates gain control in an olfactory circuit. Nature 452:956-960.

Ormerod KG, Krans JL, Mercier AJ (2015) Cell-selective modulation of the Drosophila neuromuscular system by a neuropeptide. Journal of neurophysiology 113:1631-1643.

Park D, Taghert PH (2009) Peptidergic neurosecretory cells in insects: organization and control by the bHLH protein DIMMED. General and comparative endocrinology 162:2-7.

Park D, Veenstra JA, Park JH, Taghert PH (2008a) Mapping peptidergic cells in Drosophila: where DIMM fits in. PloS one 3:e1896.

Park D, Li P, Dani A, Taghert PH (2014) Peptidergic cell-specific synaptotagmins in Drosophila: localization to dense-core granules and regulation by the bHLH protein DIMMED. The Journal of neuroscience : the official journal of the Society for Neuroscience 34:13195-13207.

Park D, Shafer OT, Shepherd SP, Suh H, Trigg JS, Taghert PH (2008b) The Drosophila basic helix-loophelix protein DIMMED directly activates PHM, a gene encoding a neuropeptide-amidating enzyme. Molecular and cellular biology 28:410-421.

Patz S, Grabert J, Gorba T, Wirth MJ, Wahle P (2004) Parvalbumin expression in visual cortical interneurons depends on neuronal activity and TrkB ligands during an Early period of postnatal development. Cerebral cortex 14:342-351.

Peng H, Ruan Z, Long F, Simpson JH, Myers EW (2010) V3D enables real-time 3D visualization and quantitative analysis of large-scale biological image data sets. Nat Biotechnol 28:348-353.

Pitkow X, Meister M (2012) Decorrelation and efficient coding by retinal ganglion cells. Nature neuroscience 15:628-635.

Pratt GE, Farnsworth DE, Fok KF, Siegel NR, McCormack AL, Shabanowitz J, Hunt DF, Feyereisen R (1991) Identity of a second type of allatostatin from cockroach brains: an octadecapeptide amide with a tyrosine-rich address sequence. Proceedings of the National Academy of Sciences of the United States of America 88:2412-2416.

Predel R, Rapus J, Eckert M (2001) Myoinhibitory neuropeptides in the American cockroach. Peptides 22:199-208. 
Raj A, van Oudenaarden A (2008) Nature, nurture, or chance: stochastic gene expression and its consequences. Cell 135:216-226.

Reichwald K, Unnithan GC, Davis NT, Agricola H, Feyereisen R (1994) Expression of the allatostatin gene in endocrine cells of the cockroach midgut. Proceedings of the National Academy of Sciences of the United States of America 91:11894-11898.

Reisenman CE, Dacks AM, Hildebrand JG (2011) Local interneuron diversity in the primary olfactory center of the moth Manduca sexta. Journal of comparative physiology A, Neuroethology, sensory, neural, and behavioral physiology 197:653-665.

Root CM, Masuyama K, Green DS, Enell LE, Nässel DR, Lee CH, Wang JW (2008) A presynaptic gain control mechanism fine-tunes olfactory behavior. Neuron 59:311-321.

Salio C, Lossi L, Ferrini F, Merighi A (2006) Neuropeptides as synaptic transmitters. Cell Tissue Res 326:583-598.

Saraswati S, Adolfsen B, Littleton JT (2007) Characterization of the role of the Synaptotagmin family as calcium sensors in facilitation and asynchronous neurotransmitter release. Proceedings of the National Academy of Sciences of the United States of America 104:14122-14127.

Schachtner J, Trosowski B, D'Hanis W, Stubner S, Homberg U (2004) Development and steroid regulation of RFamide immunoreactivity in antennal-lobe neurons of the sphinx moth Manduca sexta. The Journal of experimental biology 207:2389-2400.

Schafer S, Bicker G (1986) Distribution of GABA-like immunoreactivity in the brain of the honeybee. The Journal of comparative neurology 246:287-300.

Seidel C, Bicker G (1997) Colocalization of NADPH-diaphorase and GABA-immunoreactivity in the olfactory and visual system of the locust. Brain research 769:273-280.

Siju KP, Reifenrath A, Scheiblich H, Neupert S, Predel R, Hansson BS, Schachtner J, Ignell R (2013) Neuropeptides in the antennal lobe of the yellow fever mosquito, Aedes aegypti. The Journal of comparative neurology.

Skaer NJ, Nässel DR, Maddrell SH, Tublitz NJ (2002) Neurochemical fine tuning of a peripheral tissue: peptidergic and aminergic regulation of fluid secretion by Malpighian tubules in the tobacco hawkmoth M. sexta. The Journal of experimental biology 205:1869-1880.

Stratmann J, Thor S (2017) Neuronal cell fate specification by the molecular convergence of different spatio-temporal cues on a common initiator terminal selector gene. PLoS genetics 13:e1006729.

Sun X, Fonta C, Masson C (1993) Odour quality processing by bee antennal lobe interneurones. Chemical senses 18:355-377.

Sweeney LB, Bikoff JB, Gabitto MI, Brenner-Morton S, Baek M, Yang JH, Tabak EG, Dasen JS, Kintner CR, Jessell TM (2018) Origin and Segmental Diversity of Spinal Inhibitory Interneurons. Neuron 97:341-355 e343.

Teal PE (2002) Effects of allatotropin and allatostatin on in vitro production of juvenile hormones by the corpora allata of virgin females of the moths of Heliothis virescens and Manduca sexta. Peptides 23:663-669.

Tritsch NX, Granger AJ, Sabatini BL (2016) Mechanisms and functions of GABA co-release. Nature reviews Neuroscience 17:139-145.

Utz S, Schachtner J (2005) Development of A-type allatostatin immunoreactivity in antennal lobe neurons of the sphinx moth Manduca sexta. Cell Tissue Res 320:149-162.

Utz S, Huetteroth W, Vomel M, Schachtner J (2008) Mas-allatotropin in the developing antennal lobe of the sphinx moth Manduca sexta: distribution, time course, developmental regulation, and colocalization with other neuropeptides. Developmental neurobiology 68:123-142.

Utz S, Huetteroth W, Wegener C, Kahnt J, Predel R, Schachtner J (2007) Direct peptide profiling of lateral cell groups of the antennal lobes of Manduca sexta reveals specific composition and changes in neuropeptide expression during development. Developmental neurobiology 67:764777.

Veenstra JA, Hagedorn HH (1995) Isolation of two AKH-related peptides from cicadas. Archives of insect biochemistry and physiology 29:391-396. 
Wichterle H, Turnbull DH, Nery S, Fishell G, Alvarez-Buylla A (2001) In utero fate mapping reveals distinct migratory pathways and fates of neurons born in the mammalian basal forebrain. Development 128:3759-3771.

Wilson RI (2013) Early olfactory processing in Drosophila: mechanisms and principles. Annual review of neuroscience 36:217-241.

Wilson RI, Laurent G (2005) Role of GABAergic inhibition in shaping odor-evoked spatiotemporal patterns in the Drosophila antennal lobe. The Journal of neuroscience : the official journal of the Society for Neuroscience 25:9069-9079.

Witten JL, Truman JW (1996) Developmental plasticity of neuropeptide expression in motoneurons of the moth, Manduca sexta: steroid hormone regulation. Journal of neurobiology 29:99-114.

Yapici N, Kim YJ, Ribeiro C, Dickson BJ (2008) A receptor that mediates the post-mating switch in Drosophila reproductive behaviour. Nature 451:33-37.

Yavorska I, Wehr M (2016) Somatostatin-Expressing Inhibitory Interneurons in Cortical Circuits. Frontiers in neural circuits 10:76. 

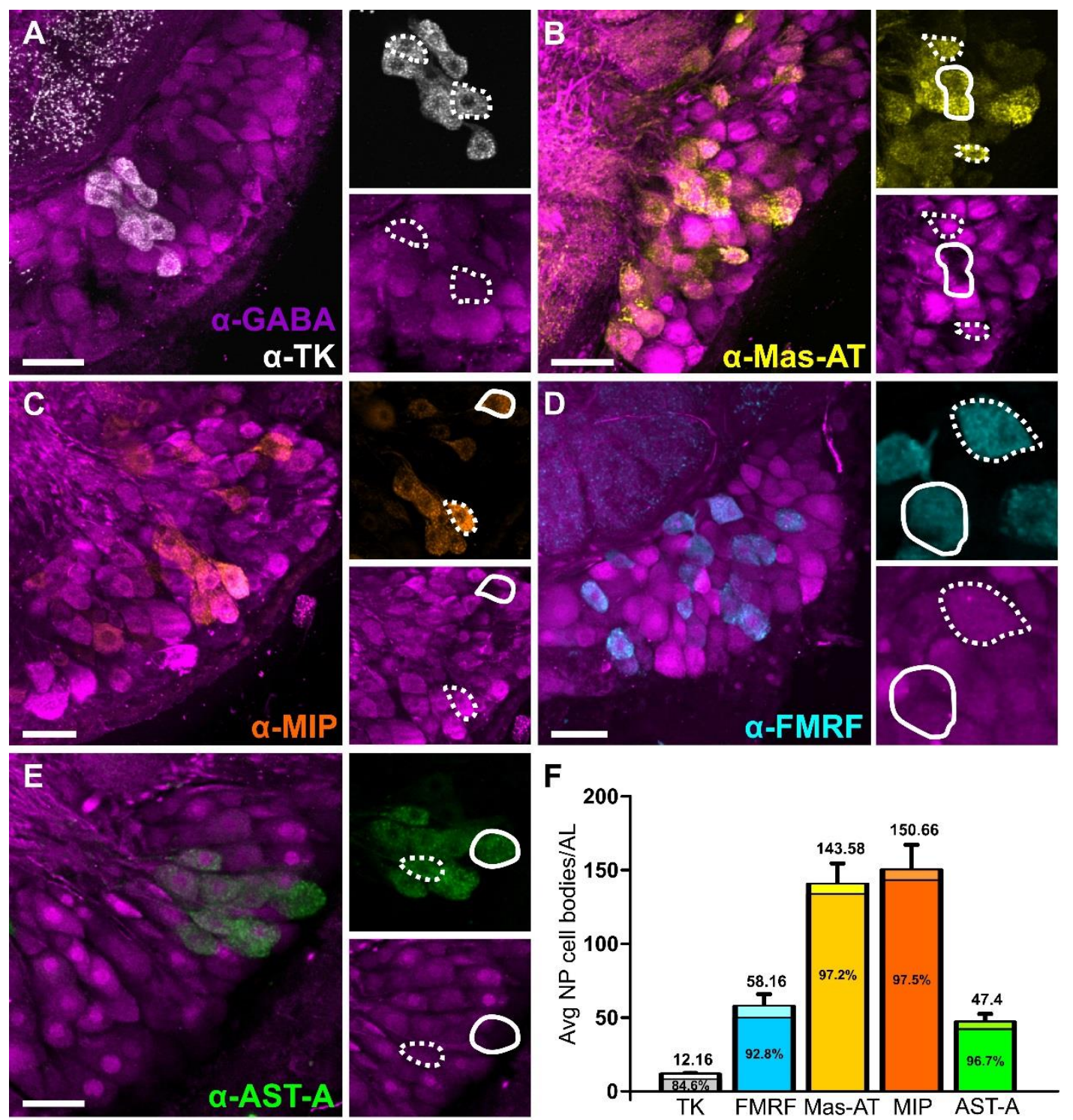

Figure 1: Peptidergic LNs predominantly co-express GABA Dashed lines = co-expressed. Solid lines = not co-expressed. A: Lateral cell cluster (LCC) labeled for GABA (magenta) and Tachykinin (TK; white). B: LCC labeled for GABA (magenta) and Allatotropin (Mas-AT; yellow). C: LCC labeled for GABA (magenta) and Myoinhibitory peptide (MIP; orange). D: LCC labeled for GABA (magenta) and FMRFamide (cyan). E: LCC labeled for GABA (magenta) and Allatostatin-A (AST-A; green). F: Bar graph of average number of cell bodies (above bars) that express each transmitter type per AL and the percentage (within bars) of each neuropeptide population per AL that co-express GABA. See Table 2 for averages and standard deviations. $\mathrm{n}=6$ animals per combination. All scale bars $=50 \mathrm{um}$. 

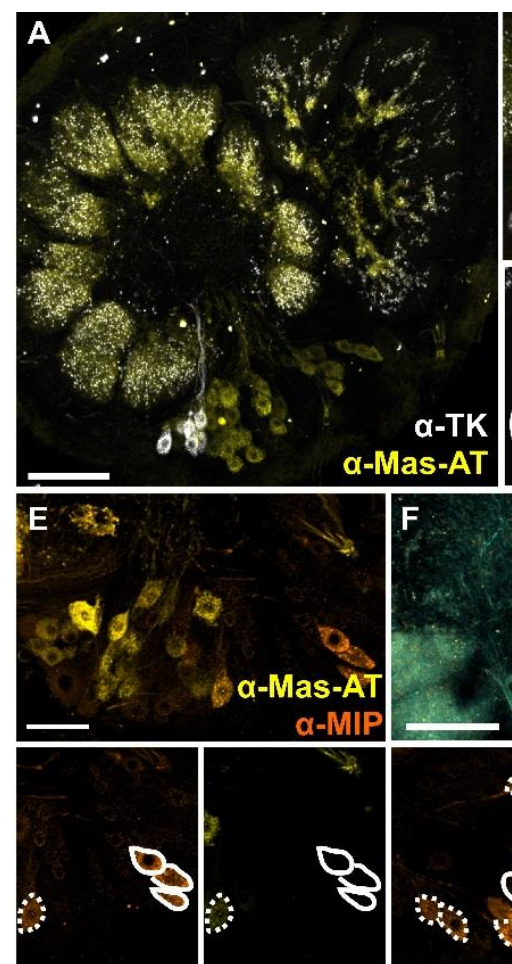
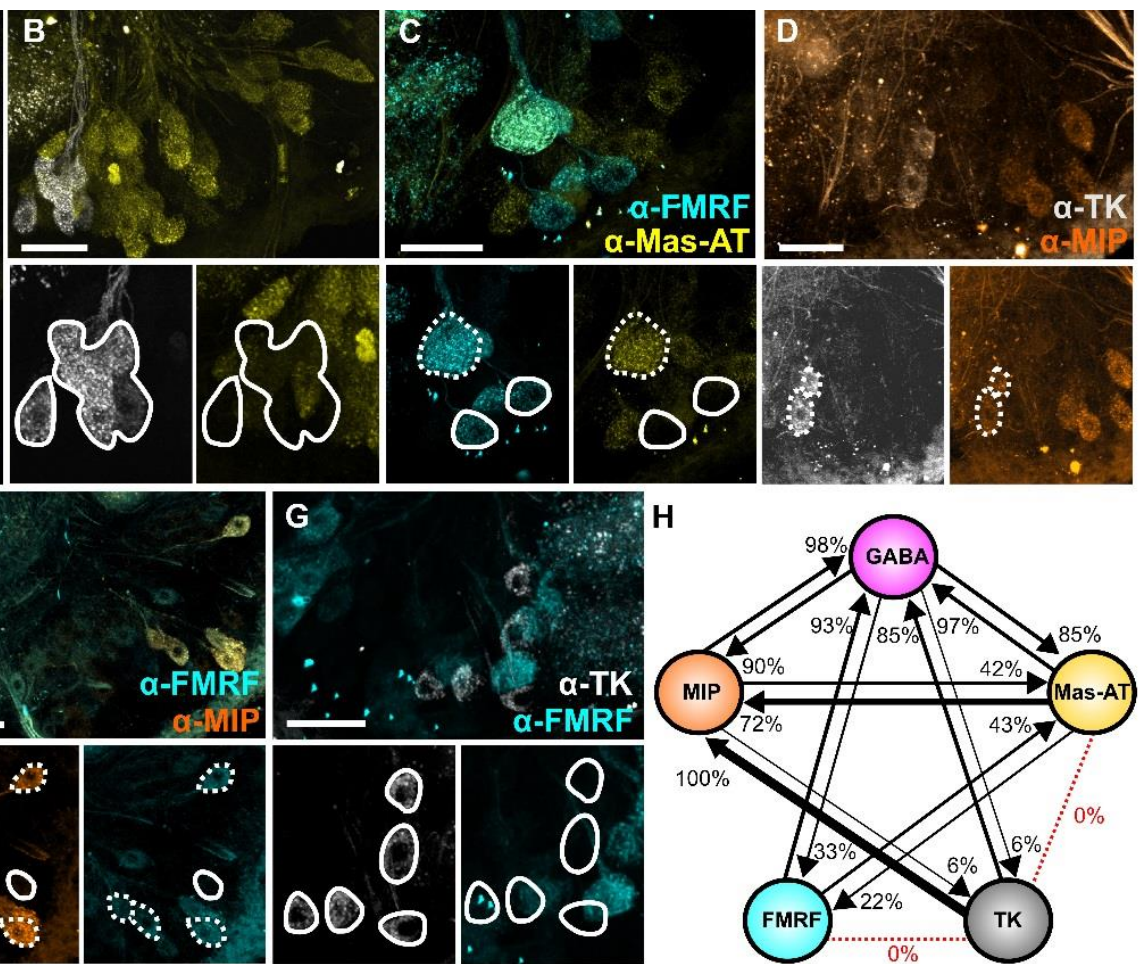

H

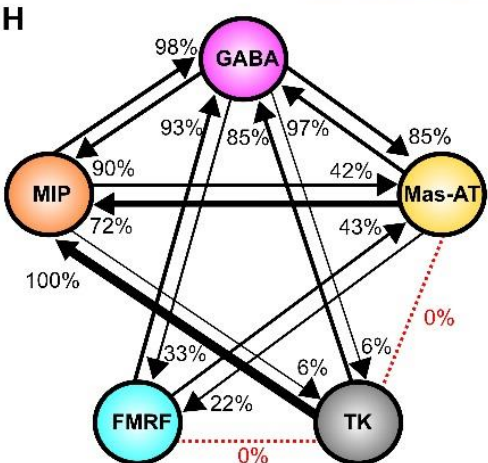

Figure 2: Neuropeptide co-expression is heterogeneous Dashed lines $=$ co-expressed. Solid lines $=$ not co-cxpressed. Co-cxpression for A,B: TK (white) and Mas-AT (yellow), C: FMRFamide (cyan) and Mas-AT, D: TK and MIP (orange), E: Mas-AT and MIP, F: FMRFamide and MIP and G: TK and FMRFamide. All scale bars =50um. H: Schematic representation of transmitter co-expression by LNs. Each circle represents the population of LNs that express a given transmitter. Arrow width and percentage located at arrowhead represent proportion of a given LN type (arrow origin) that also express a second transmitter (arrow destination). FMRFamide and MIP co-expression could not be calculated for technical reasons (see methods). No TK LNs co-expressed FMRFamide or Mas-AT. Non-GABAergic LNs are not depicted. 
A

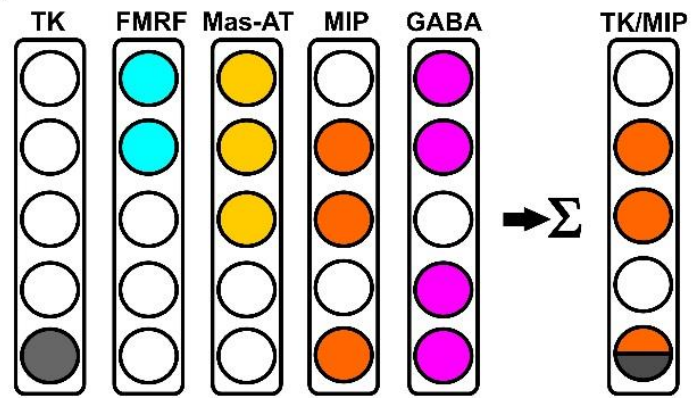

B

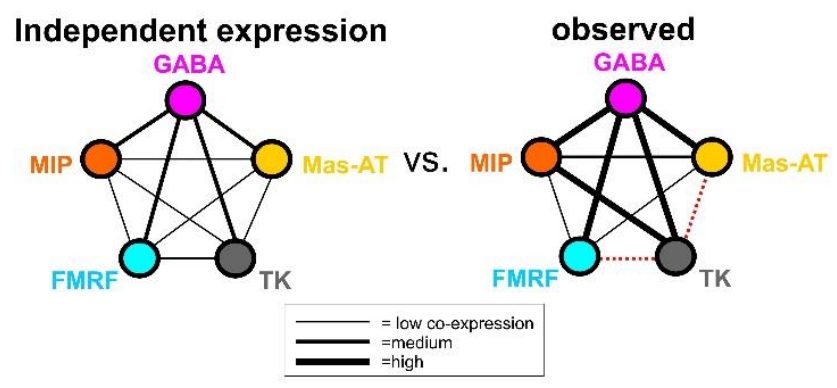

Figure 3: Schematic representations of the computational model used to calculate the probability of LN co-expression patterns A: Each column represents a transmitter, the number of rows corresponds to the total \# of neurons in the population (reduced in this illustration to 5 total cells for the sake of simplicity, $360 \mathrm{LNs}$ in reality). The number of neurons in each column that are transmitter positive correspond to the average number of neurons (standard deviations built in) that express each transmitter observed using immunocytochemistry (see Figure 1F and Table 1 for values). The model then sums across each row in a pairwise fashion to determine the co-expression percentage of a given transmitter pair. For example, for TK/MIP, the model would predict that $1 / 3$ of MIP neurons (orange) would co-express TK assuming independent probabilities of expression for each transmitter. B: Schematic representation comparing predicted co-expression percentages from the "independent expression" model to our observed co-expression patterns. Each circle represents a population of LNs that express a given transmitter. Given the number of neurons that express each individual transmitter (Table 1), the model calculates the probability that a neuron will co-express two transmitters. Line thickness represents degree of transmitter co-expression. We then compare the predicted coexpression from our "independent expression" model to our observed co-expression values to see if observed co-expression can be explained based on independent probability of expression . 
A

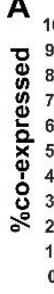

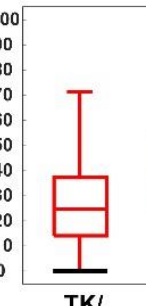
Mas-AT

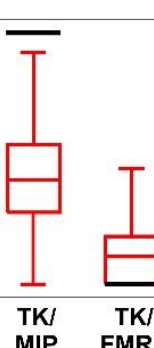

FMRF

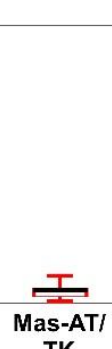

TK

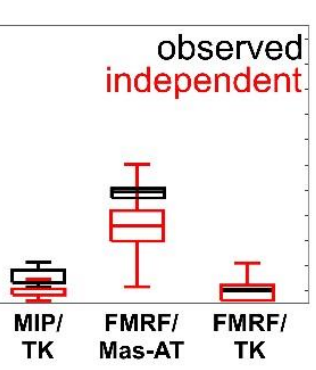

Figure 4: Computational analysis of transmitter co-expression reveals that independent expression probability cannot explain observed transmitter co-expression in LNs A: Predicted co-expression percentages for every pairwise relationship from the independent co-expression model (red) vs. observed co-expression percentages (black). A model that assumes independent probability of co-expression could not replicate observed co-expression percentages. B: Statistical comparison of the independent co-expression model's prediction vs. observed co-expression reveals that independent probability of co-expression alone cannot replicate observed LN co-expression patterns. Each colored rectangle represents an individual pair-wise relationship (e.g TK/ATR). Standard deviation indices (SDI) were calculated for every pairwise relationship to determine how closely the model could replicate observed co-expression. An SDI of 0 (blue) denotes no statistical difference between observed co-expression and predicted co-expression from the model, thus representing co-expression relationships that the model was able to replicate very well. SDI values above 1 indicate a poor match between the model and observed values.

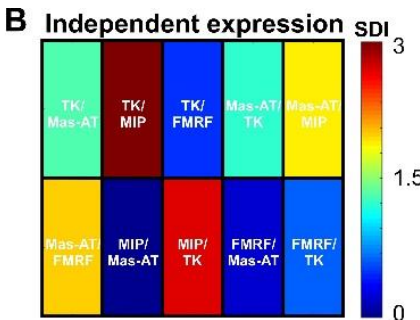

.

.

\section{.}



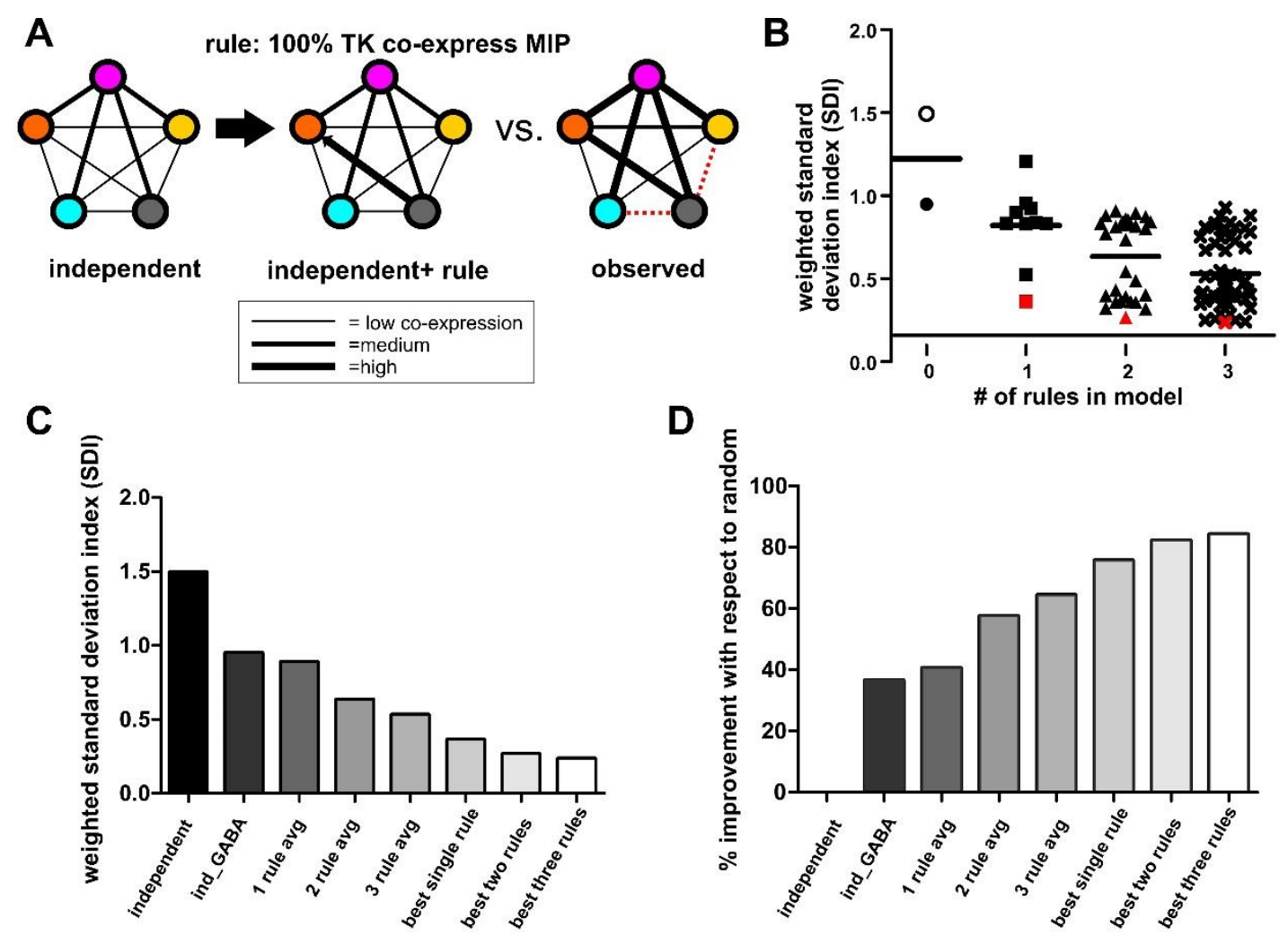

Figure 5: A few specific co-expression constraints allow replication of overall coexpression patterns A: Model constraints are applied to explicitly set the probability of a coexpression relationship to its observed value. In this example, a constraint is set in which $100 \%$ of TK LNs co-express MIP, while leaving the remaining relationships to emerge through probabilistically independent expression. This model is then compared to observed co-expression data. B: Specific rules outperform others at replicating observed co-expression patterns. Open circle represents model run with total number of LNs set to 360. Closed symbols represent models runs with total number of neurons set to the total number of GABAergic LNs (180). Red denotes standout iterations of the model that best replicated observed co-expression. The single rule that shifted the prediction closest to observed coexpression was when the proportional relationship between MIP/Mas-AT was set as a static rule in the model (red square). The two rules that shifted the prediction closest to observed coexpression were MIP/Mas-AT and TK/Mas-AT. The three rules that shifted the prediction closest to observed co-expression were TK/Mas-AT + Mas-AT/MIP + FMRF/Mas-AT. C: Weighted SDI values for various model iterations. The model improves as more rules are added. D: Percent improvement of each model's predictive power with respect to the independent expression model. Both GABA constraint and MIP/Mas-AT rule drastically improved the model's ability to replicate co-expression patterns. Note that the MIP/Mas-AT rule model even outperformed the average prediction of all models containing three rules. 
A

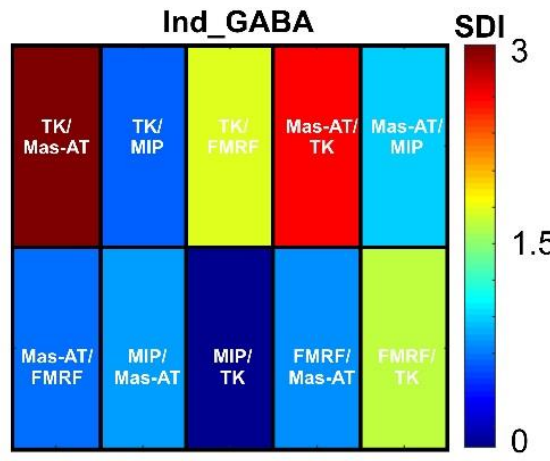

C

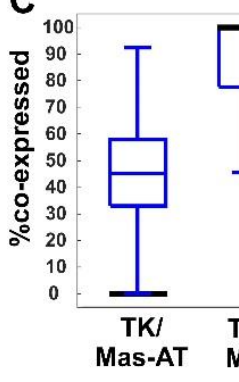

D

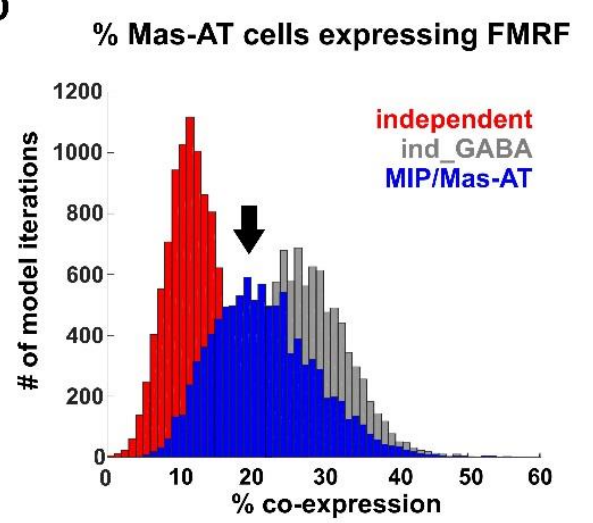

B

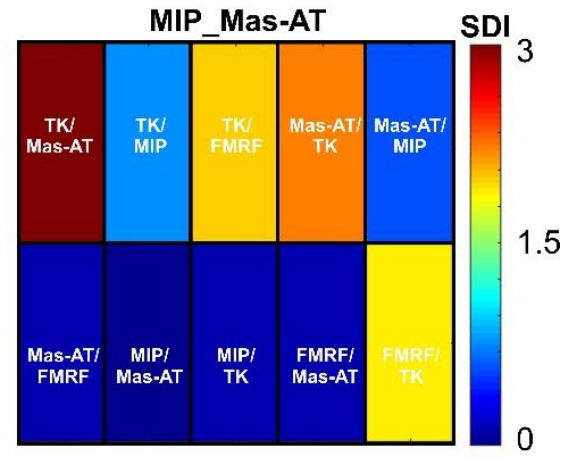

1.5

Figure 6: MIP/ATR co-expression rule best biases the model to replicate observed coexpression patterns A: Reducing the total number of neurons in the model to the total number of GABAergic LNs (180), improves model performance. SDI $=0$ (blue) denotes no statistical difference between observed co-expression and predicted co-expression. SDI $>1$ indicates a poor match between the model and observed values. B: Constraining the model based on MIP/Mas-AT co-expression causes the model to reliably replicate many observed co-expression patterns. A model following this single rule outperformed the average of all models containing three set co-expression rules. C: All predicted pairwise co-expression percentages from the model following the MIP/Mas-AT rule (blue) vs. observed coexpression percentages (black). D: Neither independent (red), nor ind_GABA (grey) models reliably replicated observed co-expression patterns (Mas-AT/FMRF used as an example). However, the MIP/Mas-AT (blue) constraint best replicates observed co-expression patterns (denoted by black arrow). E: Observed TK co-expression patterns (TK/Mas-AT used an example) were not reliably replicated by any model iteration; independent expression model prediction (red), ind_GABAmodel (grey), and MIP/Mas-AT model prediction (blue). 


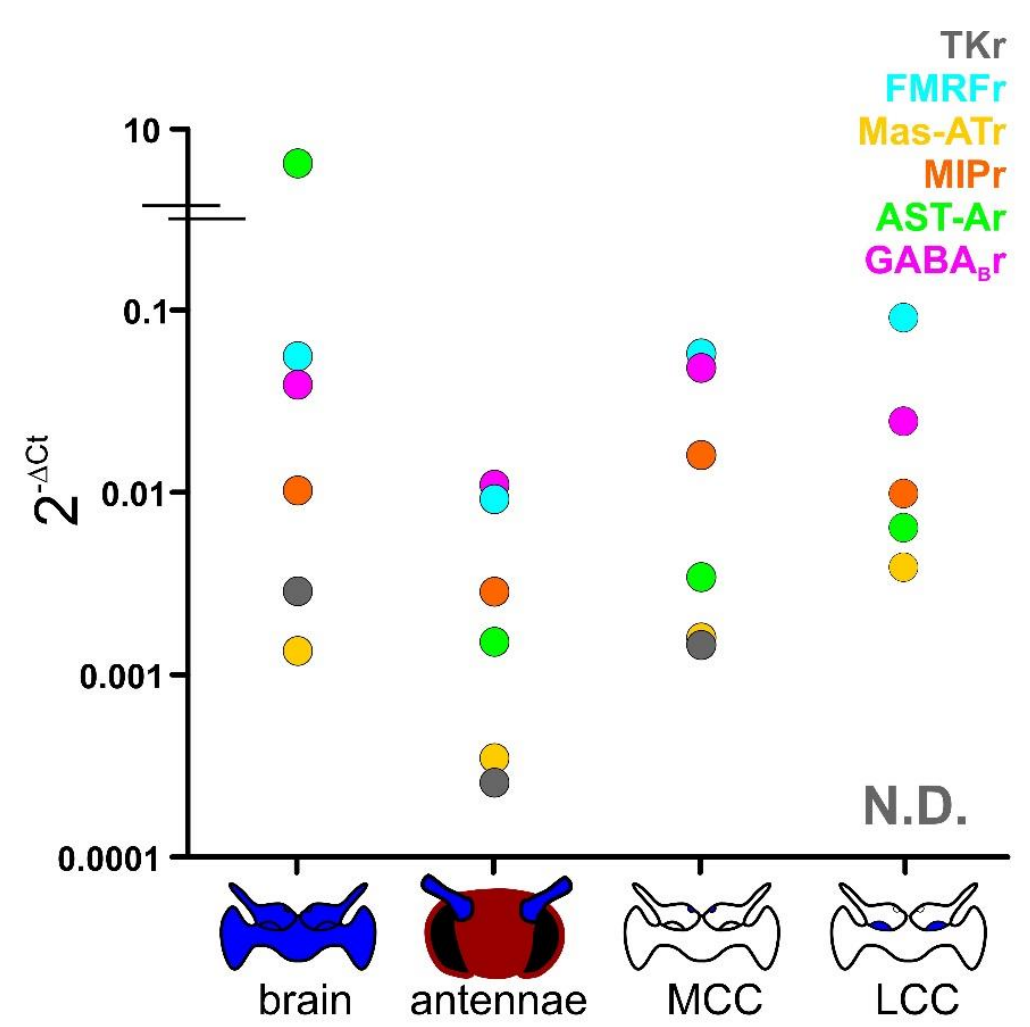

Figure 7: Neuropeptide and $G A B A_{B}$ receptor expression across principal neuron types of the AL. Relative receptor expression for Mas-ATr, MIPr, ASTr, FMRFr, GABA ${ }_{B} r$ are present in all tissue types and therefore expressed in ORNs, LNs and PNs in varying expression levels. Cartoons on the $\mathrm{x}$-axis represent the tissue type (blue) used to extract mRNA from each population of principal olfactory cell types. TKr was not detectable (N.D.) in lateral cell cluster mRNA and therefore not detectable in LNs. RpS3 was used as the reference gene. See Table 2 for primer sequences and Table 3 for raw $\mathrm{Cq}$ values for all receptors. 


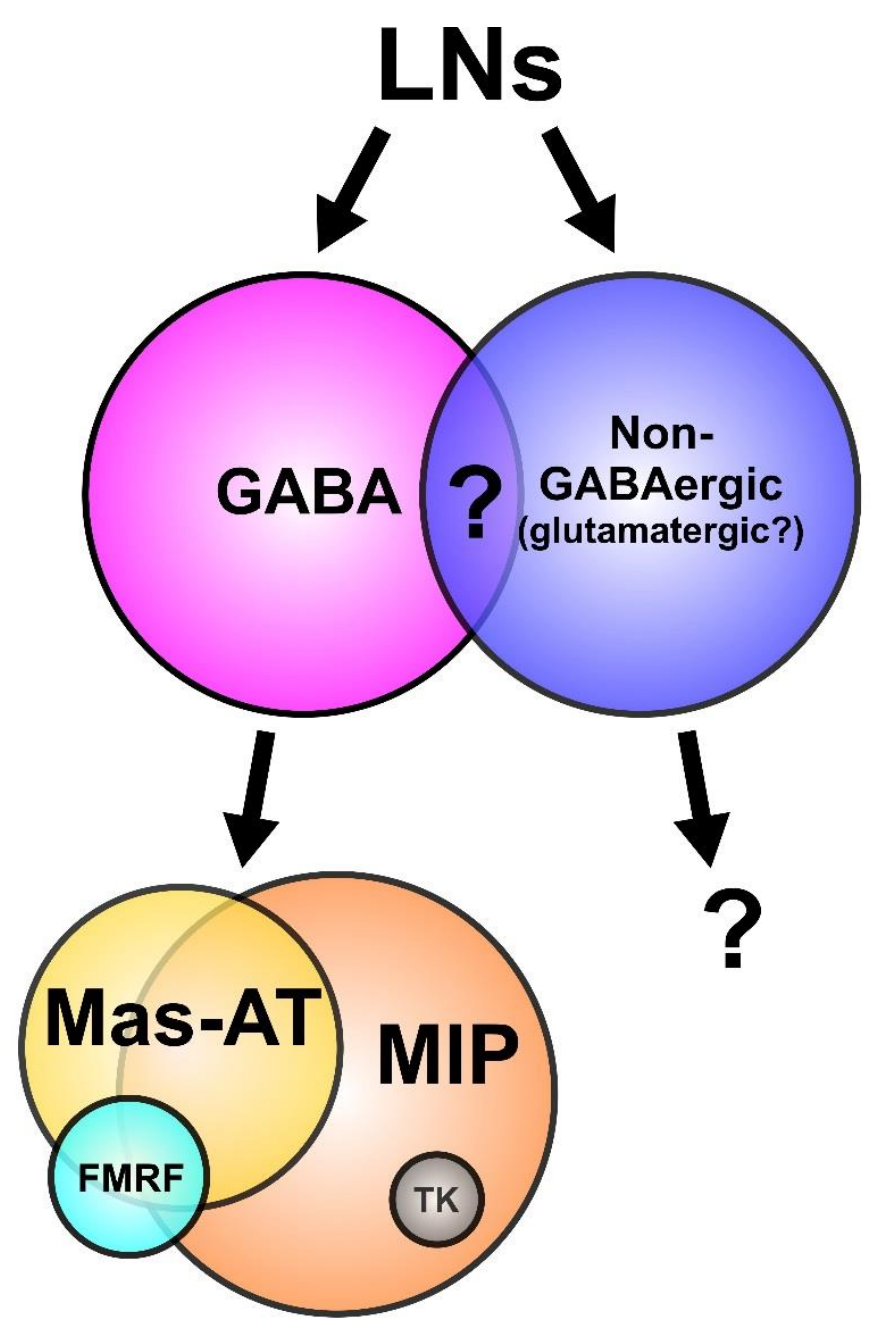

Figure 8: Heterogeneous transmitter co-expression in LNs blurs sub-divisions While LNs can be broadly subdivided based on small transmitter (GABA vs. non-GABAergic (glutamatergic?)), co-expression within the GABAergic class reveals that LNs sub-classes cannot be identified on individual transmitter expression alone. Neuropeptide co-expression in the $\mathrm{AL}$ is both heterogeneous and partially overlapping across the entire population rather than consistent within specific subpopulations of LNs. 
CHAPTER 5

Discussion and Future Directions 
(portions of this chapter are based on my publication "Lizbinski, K. M., \& Dacks, A. M. (2018). Intrinsic and extrinsic neuromodulation of olfactory processing. Frontiers in cellular neuroscience, 11, 424.)

My dissertation explores the anatomical, physiological and mechanistic basis by which a sensory system integrates the influence of multiple extrinsic and intrinsic modulators. In Chapter 2, I describe a detailed map of serotonin (5-HT) and dopamine (DA) distribution with relation to functional classes of antennal lobe (AL) neurons, providing the anatomical basis for integration of two extrinsic modulators on olfactory processing. In Chapter 3, I demonstrate that while 5-HT and DA simultaneously physiologically converge onto common AL neurons, they diverge by altering different odor-evoked response features to modulate olfactory processing via distinct mechanisms. In Chapter 4, I provide an atlas of heterogeneous transmitter co-expression in AL local interneurons, putative neuropeptide and GABAb receptor distribution in the olfactory system, and finally, develop an analytical tool to assess heterogeneity in any neural population. Overall, I demonstrate that the AL integrates the influence of two extrinsic modulators within single neurons to mediate network-wide changes in response to odors, and local interneurons intrinsic to the olfactory circuit release a heterogeneous mix of transmitters that result in a dynamic cocktail of modulators that have the potential to target every level of processing within the AL.

Due to the ubiquity of neuromodulation across all neural networks, the general principles outlined here likely apply to other systems. Specifically, there are many parallels between the insect and vertebrate olfactory systems (Hildebrand and Shepherd, 1997; Ache and Young, 2005; Lizbinski and Dacks, 2018), including the diverse sources of extrinsic and intrinsic modulation. Here I will briefly detail the similarities between insect and vertebrate olfactory systems to highlight the conserved mechanisms and roles of extrinsic and intrinsic neuromodulation across species. I will then discuss the future directions and importance of my findings within the broad field of neuroscience.

\section{Serotonergic modulation of olfactory processing}

Much like the widely projecting serotonergic neurons in insect olfactory systems, Raphe neurons release 5-HT and project throughout the brain of vertebrates. The olfactory bulb is organized into discrete layers which are innervated by different functional classes of neurons (Shipley and Ennis 1996). This stereotyped organization allows modulatory neurons to 
differentially target different cell-types based on their projections within different layers of the bulb. For example, the glomerular layer of the olfactory bulb which contains mostly juxtaglomerular local interneurons, receives strong serotonergic innervation from a large number of Median and Dorsal Raphe neurons (Pinching and Powell, 1971; McLean and Shipley, 1987; Shipley and Ennis, 1996; Gomez et al., 2005; Steinfeld et al., 2015; Suzuki et al., 2015; Muzerelle et al., 2016). Thus, consistent with my finding that 5-HT strongly modulates LNs in the insect AL, 5-HT alters local processing cells to modulate lateral interactions within the vertebrate olfactory bulb. Additionally, sub-populations of Dorsal Raphe neurons exhibit distinct physiological effects on the network and have differing, yet parallel connectivity within the network (Ren et al., 2018). Collectively, this demonstrates that modulatory neurons can have differing effects on the network based on their location and the functional cell-types they target. Raphe neurons also have a relatively heterogeneous transcriptional profile (Okaty et al., 2015) and individual Raphe neurons can respond to either reward or punishing stimuli (Nakamura et al., 2008; Ranade and Mainen, 2009; Bromberg-Martin et al., 2010; Miyazaki et al., 2011a; Miyazaki et al., 2011b; Nakamura, 2013; Liu et al., 2014; Pollak Dorocic et al., 2014; Weissbourd et al., 2014; Cohen et al., 2015; Hayashi et al., 2015; Li et al., 2016; Luo et al., 2016), as well as display experience dependent plasticity in response properties (Zhong et al., 2017). Furthermore, pharmacological studies have suggested that 5-HT facilitates odor preference learning in rat pups (McLean et al., 1993; McLean et al., 1996; Langdon et al., 1997; Price et al., 1998; Yuan et al., 2003). Thus 5-HT plays a variety of roles in olfactory learning and modulation in vertebrates, demonstrating the difficulty of prescribing a unified role to this important signaling molecule.

Similar to my findings that 5-HT exhibits variable effects on odor-evoked firing rate of AL neurons, neurons in the olfactory bulb also exhibit heterogeneous serotonergic effects within and across functional types of olfactory bulb neurons. Early studies in the rabbit olfactory bulb showed that application of 5-HT decreased spontaneous firing rate of MCs (Bloom et al., 1964). More recent studies in rats revealed that 5-HT can directly (via the 5-HT2a receptor) and indirectly (via 5-HT2a receptor expression in external tufted cells) excite mitral cells, yet also increases inhibition exerted upon mitral cells by depolarizing a subset of juxtaglomerular cells (via 5-HT2c) (Hardy et al., 2005; Petzold et al., 2009; Liu et al., 2012; Schmidt and Strowbridge, 2014; Brunert et al., 2016; Huang et al., 2017). Stimulating Raphe input specifically to the 
olfactory bulb depolarizes a subset of output neurons called tufted cells (Kapoor et al., 2016) and has a heterogeneous effect on mitral cell baseline activity (Brunert et al., 2016; Kapoor et al., 2016). However, as discussed below, dual-transmission of glutamate and 5-HT by Raphe neurons complicates these findings (Liu et al., 2014). Raphe stimulation appears to predominantly enhance mitral cell odor-evoked responses (Brunert et al., 2016), however this can be odor dependent (Kapoor et al., 2016). Consistent with a theme of heterogeneity, 5-HT was recently demonstrated to excite mitral cells in the main olfactory bulb, yet inhibit mitral cells in the accessory olfactory bulb (Huang et al., 2017). Altogether the heterogeneous effects of 5HT on functional classes of olfactory bulb neurons is likely mediated by cell-class specific receptor expression. While there are only four 5-HT receptors in the AL of M. sexta, there are at least ten 5-HT receptors expressed in the olfactory bulb (Appel et al., 1990; Hellendall et al., 1993; Shen et al., 1993; Tecott et al., 1993; Watts et al., 1994; McLean et al., 1995; Wright et al., 1995; Waeber et al., 1998; Bai et al., 2004; Lucaites et al., 2005; Petzold et al., 2009). This expands the complexity by which a single modulator can alter olfactory process across multiple cell types. Overall, 5-HT exhibits heterogeneous effects on the olfactory system of insects and vertebrates, demonstrating that future studies must take receptor expression, within-cell class diversity, and the diversity of modulatory neurons themselves, into account.

\section{Dopaminergic modulation of olfactory processing in a broad context}

Similar to serotonergic modulation, dopaminergic modulation is heterogeneous across multiple parameters within both the olfactory system and across organisms (Vinauger et al 2018, Dacks et al 2013). DA can be both intrinsic (Kirchhof et al., 1999; Hamanaka et al., 2016; Tedjakumala et al., 2017), and extrinsic (Dacks et al., 2012), depending on the organism. In $M$. sexta, the extrinsic dopamine neurons, the DAARs, innervate all glomeruli, however in other insects like the mosquito, the degree to which each glomerulus was innervated by DA varied (Vinauger et al 2018). This suggests that DA may selectively modulate certain odors more (or less) than others. Within the AL of M. sexta, DA is associated with the context of aversive stimuli, as DA receptor antagonists block aversive (Dacks et al., 2012), but not appetitive conditioning (Riffell et al., 2013). In mosquitos, manipulating D1 receptors using D1 receptor antagonists, D1 receptor specific RNAi, and whole body CRISPR-Cas9 against D1, prevents olfactory learning (Vinauger et al 2018). Specifically, for RNAi experiments, D1 receptor manipulation affected the 
mosquitoes' ability to learn aversive information but did not affect innate olfactory behavior. This evidence, along with behavioral work in M. sexta on the basis of DA and aversion on olfactory learning (Dacks et al., 2012), demonstrates that DA is likely operates in the context of aversion. Both the AL and the mushroom bodies, the center for learning and memory in insects, likely mediate this aversion, as dopamine plays a central role in olfactory learning (Unoki et al., 2005; Schwaerzel et al 2003; Kim et al, 2007; Schroll et al., 2006). Past studies have shown that in $M$. sexta DA enhances odor-evoked responses of extracellularly recorded AL neurons (Dacks et al., 2012, Vinauger et al., 2018), but did not affect response gain (Dacks et al., 2012), unlike 5-HT (Dacks et al., 2008). My results are consistent with the finding that DA does not affect response gain, however, the effects of DA were variable across cell-type, with subsets of neurons either enhanced or inhibited by DA depending on the cell. This suggests that, like 5-HT, AL neurons likely exhibit variable expression of DA receptors within each functional cell class.

Unlike the extrinsic source of DA in the AL of $M$. sexta, in the olfactory bulb, subsets of GABA/DAergic juxtaglomerular neurons (Kosaka and Kosaka, 2016) synapse onto other local processing neurons in the bulb, providing an intrinsic source of DA (Aungst et al., 2003; Kiyokage et al., 2010; Liu et al., 2013). Multiple sub-sets of DAergic neurons make up this population, thus much like AL local interneurons, DAergic neurons are highly diverse and many co-express GABA (Kosaka and Kosaka, 2016, Aungst et al., 2003; Kiyokage et al., 2010; Liu et al., 2013). This heterogeneous mix of juxtaglomerular neurons mediates presynaptic inhibition of olfactory receptor neurons (ORNs) via activation of inhibitory D2 and GABAb receptors, thereby reducing olfactory input to mitral and tufted cells. Specifically, electron microscopy and anatomical studies in rats revealed metabotropic $\mathrm{GABAb}$ and $\mathrm{D}_{2}$ receptor expression on vertebrate ORNs (Bonino et al., 1999; Koster et al., 1999) and direct physiological evidence demonstrated that dopamine modulates the olfactory nerve synapse (Hsia et al., 1999; Berkowicz and Trombley, 2000). My anatomical findings in Chapter 2, suggest that DA alone extends far enough distally within glomeruli to modulate ORN synaptic input. Thus, work in vertebrates demonstrating DA mediated presynaptic inhibition, may also hold true for M. sexta.

\section{Intrinsic modulation of olfactory processing by local interneurons}


Heterogeneous populations of LNs, in both the insect and vertebrate olfactory system, are the main source of intrinsic modulation, altering network processing within the context of previous and ongoing activity. LNs perform many tasks including presynaptic gain control, divisive normalization, lateral excitation and inhibition, regulating spike timing by promoting synchrony, and modulating dynamic control of response range (reviewed in Martin et al., 2011 and Wilson et al, 2013). In Chapter 4, I find that LNs of M. sexta are highly heterogeneous, releasing up to four individual neuropeptides and GABA. Furthermore, co-expression of peptides is variable across the entire across the entire population. In the olfactory bulb, periglomerular and external tufted cells also appear to express a wide variety of neuropeptides, including NPY, VIP and CCK (Seroogy et al., 1985; Gall et al., 1986). However, very little is known about how peptides influence in olfactory processing, particularly in vertebrates. Additionally, many vertebrate cell-types are still broadly defined based on the presence of single markers, like peptides or other neurotransmitters. This is misleading, because traits like transmitter co-expression are often partially overlapping over previously defined 'distinct' sub-sets of neurons. To avoid incorrectly sub-categorizing neurons, and understand their full role within a network, detailed analyses of co-expression (of multiple traits) are necessary. Below I discuss how the field should move forward to assess the important yet understudied world of peptides.

\section{Future directions}

\section{Anatomy}

In Chapter 2, I provide the anatomical framework for the convergence of modulatory neurons onto the functional neuron types of the AL. In summary, I find that 5-HT and DA have the potential to simultaneously affect LNs and PNs, while DA alone likely synaptically interacts with ORNs. While relative close association is not proof of synaptic relationships, my anatomical work identified potential shared targets of 5-HT and DA modulation (which I determined the physiological basis for in Chapter 3). Recent advances in connectomics have provided a previously unmatched level of precision of the anatomy of many nervous systems, including a whole EM data set of the Drosophila melanogaster larval olfactory system (Berck et al., 2016) and adult D. melanogaster whole brain (Zheng et al., 2018). However, the connectome is not enough (Bargmann, 2012) as neuromodulation drastically changes the functional connectivity of neural circuits (Getting, 1989). While these resources are not yet available for $M$. sexta, my work along with the recent advances in connectomics raise important 
future questions. Do 5-HT and DA neurons directly synapse onto LNs, PNs and ORNs or are their potential effects mediated by polysynaptic interactions? Are there glomerular differences in synaptic connectivity for each modulator? While both 5-HT and DA innervated all isomorphic glomeruli, each modulator may have greater influence in certain glomeruli, as is seen for DA in the AL of mosquitos (Vinauger et al., 2018) and for 5-HT in D. melanogaster (Coates et al., 2017). These differences in innervation could mean that 5-HT and DA differentially modulate certain odors more (or less) than others.

Finally, we find that 5-HT does not extend far enough distally within glomeruli into the layer occupied by ORNs, suggesting that the CSDns do not synaptically interact with ORNs. This is consistent with work in the D. melanogaster AL showing that the CSDns do not synapse onto ORNs (Coates et al., 2017). However, previous studies have found that presynaptic inhibition of ORNs is enhanced by 5-HT despite 5-HT having no direct effect on ORNs (Dacks et al., 2009) and ORNS in D. melanogaster consistently express the 5-HT2B receptor (Sizemore and Dacks, 2016), suggesting that 5-HT modulates ORN activity either through lateral interactions or directly via non-synaptic sources. ORNs are continuously subjected to the influence of circulating neuromodulators in the hemolymph (Schendzielorz et al., 2015) and the mRNA for four of the $M$. sexta 5-HT receptors are expressed in the antennae, far from the axons of CSD neurons in the AL (Dacks et al., 2006b; Dacks et al., 2013), although it is possible that 5HT release from the CSDns may act at a distance to influence ORNs in an as yet unobserved manner. Therefore, future work should determine the contribution of both the synaptic and nonsynaptic sources of neuromodulation within the AL.

Using extracellular tetrode recordings of AL neurons, I determined that while 5-HT and DA converge onto many of the same AL neurons, they diverge by affecting different phases of odor-evoked responses via distinct mechanisms. I also found the effects of both 5-HT and DA were variable within LNs and PNs, such that odor-evoked activity of some neurons from each functional class were inhibited by the modulators, while some were enhanced. This suggests that subsets of neurons exhibit differential 5-HT and DA receptor expression. In order to fully understand the mechanistic basis by which modulators alter olfactory processing, future work should identify the distribution of the 5-HT and DA receptors within LNs and PNs. Using paired in situ to determine receptor expression and immunocytochemistry to highlight broad cell-classes will provide a map of modulatory receptor expression across and within AL neuron types. 


\section{Physiology}

In Chapter 3, I found that 5-HT and DA likely modulate olfactory processing via distinct mechanisms. I propose that DA likely modulates the fidelity with which PNs respond to odor stimuli by decreasing intrinsic inhibitory phases of PNs, while 5-HT alters the sensitivity of AL neurons by increasing response duration of PNs and altering lateral interactions of LNs. Future work could test the effects of DA on the ability of the olfactory system to encode rapidly fluctuating odor stimuli. While I identified a potential role for 5-HT in AL sensitivity, the role of 5-HT and DA in odor-discrimination is still an open question. Do 5-HT and DA change the AL ability to discriminate between similar and dissimilar odors? Furthermore, my work took a unique approach to determine the effects of modulators on individual functional classes of AL neurons. While I was able to definitively define neurons as PNs, my experimental design does not allow me to definitively categorize LNs based on their odor-evoked activity alone. Therefore, future studies could assess modulatory integration on these two distinct neuron types using intracellular recordings and then filling the cells to identify them as either PNs or LNs. Furthermore, my analyses could be strengthened by assessing whether each modulator changes the population response of AL neurons. For example, past studies have assessed the odor-evoked responses of AL neuron ensembles to determine similarity of odor representations; the greater the distance in Euclidian space, the more dissimilar the responses which strongly correlates with behavioral discrimination (Daly et al., 2001, 2007; Dacks et al., 2008). Using principle components analysis (PCA) and Euclidian distance, future work should determine whether modulators change the representation of AL neurons in Euclidian space for both odor sensitivity and discrimination experiments. My experimental design also does not allow me to distinguish between direct and indirect modulation on AL neurons. Therefore, future work should assess this question in an organism where cell-type specific receptor expression is known, and where lateral synaptic input can be altered in order to make the distinction between direct and indirect modulatory effects. Finally, I found that the integrated effects of both modulators were nonlinear on many individual AL neurons. To strengthen the idea that 5-HT and DA have non-linear effects, the odor-evoked effects of both modulators should be assessed on the population as a whole.

A major advantage of recording from $M$. sexta AL neurons is the ability to record from many neurons at once using extracellular tetrode recordings and assess the effect of modulators 
using bath application. However, bath application of modulators is not without its downfalls. While my work identifies the basic principles of modulatory integration and distinct mechanisms of 5-HT and DA in olfactory processing, I was unable to assess the consequences of endogenous release of each modulator from the extrinsic modulatory neurons within the circuit. To mimic endogenous release of both 5-HT and DA, reuptake inhibitors for 5-HT and DA could be bath applied to AL in concert with the odor-panel used in Chapter 3. This would allow for direct comparison of endogenous release vs. bath application on the odor-evoked effects of 5-HT and DA. In D. melanogaster, exogenous application of 5-HT vs endogenous release have opposite effects, suggesting that synaptic vs. non-synaptic sources of 5-HT may differentially modulate AL activity (Zhang and Gaudry, 2016). An ambitious experiment in M. sexta, using dual intracellular to drive CSDn activity and extracellular recording of PNs and LNs could determine whether endogenous activation of the CSDns exhibits different effects on odor-evoked responses than bath application in $M$. sexta.

Finally, while 5-HT and DA are broadly associated with waking state and aversion respectively, the contexts in which each modulator are released are not understood. Work in the connectome of $D$. melanogaster may provide clues as to which neurons provide input to the CSDns and DAARs. Ultimately, merging physiological, molecular, and behavioral manipulations will be necessary to determine the full context in which each modulator is released.

\section{Peptidergic modulation and co-expression}

In Chapter 4, I provide an atlas of heterogeneous transmitter co-expression in AL local interneurons, a putative map of neuropeptide receptor/GABAb receptor distribution in the olfactory system and finally develop an analytical tool to assess heterogeneity in any neural population. Single olfactory LNs release at least four neuropeptides as well as the classical transmitter GABA, thus activation of multiple LNs results in a dynamic cocktail of modulators that, based on receptor expression, have the potential to alter every level of olfactory processing within the AL. Neurotransmission and modulation by neuropeptides is an important, understudied and challenging topic. Broadly, peptidergic modulation as a topic is in its infancy due to a few broad challenges. 1) Neuropeptides are a diverse and abundant group of signaling molecules whose expression is sensitive to physiological state of the animals. For example, populations of neurons can switch the transmitters they release even after development, and 
physiological states such as hunger or mating alters the expression profiles of peptidergic neurons across multiple species. 2) The context in which peptides are released is not well understood. In order to release neuropeptides, neurons likely have to be driven harder and longer due to the distinct molecular mechanism of the dense-core vesicles that peptides are packaged in. While different forms of synaptotagmin with varying affinities for calcium have been identified (Fukuda et al, 2004; Saraswati et al., 2007; Wen et al., 2010), the mechanism that mediates dense core peptide vesicle release is still unknown. 3) The manner in which peptide expression is regulated in subsets of neurons is not fully understood. While a few transcription factors involved in broad peptide expression have been identified, more work is needed to determine how heterogenous patterns of peptide expression arise within and across functional cell types. 4). Receptor expression for peptides is completely understudied. Very few neuropeptide receptors have been fully functionally characterized. In order to understand how peptides alter neural function, a more detailed characterization of peptide receptors is in order.

Specifically, for my work, a number of questions remain concerning the physiological mechanisms, distribution, and patterns of neuropeptide co-expression in $M$. sexta. Namely, what are the physiological consequences of peptidergic modulation? Using similar experimental design from Chapter 3, bath application of synthesized peptides could be used to determine how peptidergic modulation alters odor coding. Perhaps a more elegant experiment (likely in $D$. melanogaster) would be to drive peptidergic LNs using whole cell patch and quantify their effect on network processing. Isolating the effects of individual peptides will be challenging due to the heterogeneous patterns of peptide co-expression observed across cell-types and species. Because peptides act on timescales longer than that of even slow metabotropic processing, studies must take timescale into consideration. Current and previous work on the physiological consequences on olfactory LN activation focus primarily on GABAergic or glutamatergic transmission. Only a few have investigated peptidergic processing. Small transmitters like GABA and glutamate are not the whole story, and future studies should embrace, not ignore, the complexity that heterogeneous peptidergic modulation brings to the table.

In Chapter 4, I also identified putative neuropeptide receptors in M. sexta, and determined their broad expression across ORNs, PNs and LNs in the olfactory system. An open question is whether each functional class of AL neurons exhibits within cell-class diversity of peptide receptors. Realistically, peptide receptors are likely expressed within smaller of each 
neuron type, and may be as heterogeneous as peptide co-expression itself. To explore this, adult M. sexta could be subject to different physiological states, i.e. starvation, mating, etc, and the expression patterns across functional cell types measured again using RT-qPCR. Additionally, in situ hybridization could be used to assess within cell-class distribution in both control and perturbed conditions. Finally, the information identified in Chapter 4 must be integrated with the knowledge of distinct morphological and physiological LN types for a more complete understanding of peptide co-expression in the olfactory system.

\section{Conclusion}

Both intrinsic and extrinsic modulation allow networks to alter the functional connectivity of neurons based on the current state of the animal. Intrinsic neuromodulation is exerted by neurons that are within a neural network and participate in information processing undertaken by that network, providing the context of past or ongoing network activity. Extrinsic neuromodulation is exerted by neurons that originate in independent networks, and provide context about the broader physiological state of the animal. The integration of both intrinsic and extrinsic neuromodulation thus merges both history of network activation with global context of physiological state to adjust the broad modulatory tone of the olfactory system as a whole.

Altogether, intrinsic and extrinsic modulatory mechanisms exert a heterogeneous influence due to complex patterns of modulatory receptor expression, cell-to-cell variability, and complex connectivity in the olfactory system. Ultimately, in order to understand realistic network function, the field of sensory neuroscience must understand how single neurons integrate the influence of multiple intrinsic and extrinsic neuromodulators to mediate network-wide changes for context-specific processing.

\section{Works Cited:}

Ache, B. W., and Young, J. M. (2005). Olfaction: diverse species, conserved principles. Neuron 48, $417-$ 430. doi: 10.1016/j.neuron.2005.10.022

Appel, N. M., Mitchell, W. M., Garlick, R. K., Glennon, R. A., Teitler, M., and De Souza, E. B. (1990). Autoradiographic characterization of (+-)-1-(2,5-dimethoxy-4-[125I] iodophenyl)-2aminopropane ([125I]DOI) binding to 5-HT2 and 5-HT1c receptors in rat brain. J. Pharmacol.

Exp. Ther. 255, 843-857.

Aungst, J. L., Heyward, P. M., Puche, A. C., Karnup, S. V., Hayar, A., Szabo, G., et al. (2003). Centresurround inhibition among olfactory bulb glomeruli. Nature 426, 623-629. doi: 10.1038/nature02185 
Bai, F., Yin, T., Johnstone, E. M., Su, C., Varga, G., Little, S. P., et al. (2004). Molecular cloning and pharmacological characterization of the guinea pig 5-HT1E receptor. Eur. J. Pharmacol. 484, 127-139. doi: 10.1016/j.ejphar.2003.11.019

Bargmann, C. I. (2012). Beyond the connectome: how neuromodulators shape neural circuits. Bioessays, 34(6), 458-465.

Berck, M. E., Khandelwal, A., Claus, L., Hernandez-Nunez, L., Si, G., Tabone, C. J., ... \& Samuel, A. D. (2016). The wiring diagram of a glomerular olfactory system. Elife, 5, e14859.

Berkowicz, D. A., and Trombley, P. Q. (2000). Dopaminergic modulation at the olfactory nerve synapse. Brain Res. 855, 90-99. doi: 10.1016/s0006-8993(99)02342-2

Bloom, F. E., Costa, E., and Salmoiraghi, G. C. (1964). Analysis of individual rabbit olfactory bulb

neuron responses to the microelectrophoresis of acetylcholine, norepinephrine and serotonin synergists and antagonists. J. Pharmacol. Exp. Ther. 146, 16-23.

Bonino, M., Cantino, D., and Sassoè-Pognetto, M. (1999). Cellular and subcellular localization of $\gamma$ -

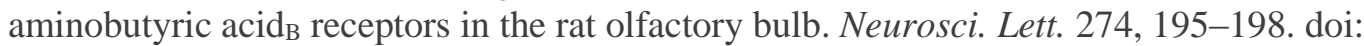
10.1016/s0304-3940(99)00697-7

Bromberg-Martin, E. S., Hikosaka, O., and Nakamura, K. (2010). Coding of task reward value in the dorsal raphe nucleus. J. Neurosci.30, 6262-6272. doi: 10.1523/JNEUROSCI.0015-10.2010

Brunert, D., Tsuno, Y., Rothermel, M., Shipley, M. T., and Wachowiak, M. (2016). Cell-type-specific modulation of sensory responses in olfactory bulb circuits by serotonergic projections from the raphe nuclei. J. Neurosci. 36, 6820-6835. doi: 10.1523/JNEUROSCI.3667-15.2016

Coates, K. E., Majot, A. T., Zhang, X., Michael, C. T., Spitzer, S. L., Gaudry, Q., \& Dacks, A. M. (2017). Identified serotonergic modulatory neurons have heterogeneous synaptic connectivity within the olfactory system of Drosophila. Journal of Neuroscience, 0192-17.

Cohen, J. Y., Amoroso, M. W., and Uchida, N. (2015). Serotonergic neurons signal reward and punishment on multiple timescales. Elife4:e06346. doi: 10.7554/eLife.06346

Dacks, A. M., Christensen, T. A., \& Hildebrand, J. G. (2006). Phylogeny of a serotonin-immunoreactive neuron in the primary olfactory center of the insect brain. Journal of Comparative Neurology, 498(6), 727-746.

Dacks, A. M., Green, D. S., Root, C. M., Nighorn, A. J., \& Wang, J. W. (2009). Serotonin modulates olfactory processing in the antennal lobe of Drosophila. Journal of neurogenetics, 23(4), 366377.

Dacks, A. M., Riffell, J. A., Martin, J. P., Gage, S. L., \& Nighorn, A. J. (2012). Olfactory modulation by dopamine in the context of aversive learning. Journal of neurophysiology, 108(2), 539-550.

Dacks, A. M., Reale, V., Pi, Y., Zhang, W., Dacks, J. B., Nighorn, A. J., \& Evans, P. D. (2013). A characterization of the Manduca sexta serotonin receptors in the context of olfactory neuromodulation. PloS one, 8(7), e69422.

Daly, K. C., Chandra, S., Durtschi, M. L., \& Smith, B. H. (2001). The generalization of an olfactorybased conditioned response reveals unique but overlapping odour representations in the moth Manduca sexta. Journal of Experimental Biology, 204(17), 3085-3095. 
Daly, K. C., Carrell, L. A., \& Mwilaria, E. (2007). Detection versus perception: physiological and behavioral analysis of olfactory sensitivity in the moth (Manduca sexta). Behavioral neuroscience, 121(4), 794.

Fukuda, M., Kanno, E., Satoh, M., Saegusa, C., \& Yamamoto, A. (2004). Synaptotagmin VII is targeted to dense-core vesicles and regulates their Ca2+-dependent exocytosis in PC12 cells. Journal of Biological Chemistry, 279(50), 52677-52684.

Gall, C., Seroogy, K. B., and Brecha, N. (1986). Distribution of VIP- and NPY-like immunoreactivities in rat main olfactory bulb. Brain Res. 374, 389-394. doi: 10.1016/0006-8993(86)90436-1

Getting, P. A. (1989). Emerging principles governing the operation of neural networks. Annual review of neuroscience, 12(1), 185-204.

Gómez, C., Briñón, J. G., Barbado, M. V., Weruaga, E., Valero, J., and Alonso, J. R. (2005).

Heterogeneous targeting of centrifugal inputs to the glomerular layer of the main olfactory bulb. $J$.

Chem. Neuroanat. 29, 238-254. doi: 10.1016/j.jchemneu.2005.01.005

Hardy, A., Palouzier-Paulignan, B., Duchamp, A., Royet, J. P., and Duchamp-Viret, P. (2005). 5Hydroxytryptamine action in the rat olfactory bulb: in vitro electrophysiological patch-clamp recordings of juxtaglomerular and mitral cells. Neuroscience 131, 717-731. doi: 10.1016/j.neuroscience.2004.10.034

Hayashi, K., Nakao, K., and Nakamura, K. (2015). Appetitive and aversive information coding in the primate dorsal raphé nucleus. J. Neurosci. 35, 6195-6208. doi: 10.1523/JNEUROSCI.286014.2015

Hellendall, R. P., Schambra, U. B., Liu, J. P., and Lauder, J. M. (1993). Prenatal expression of 5$\mathrm{HT}_{1 \mathrm{C}}$ and 5- $\quad \mathrm{HT}_{2}$ receptors in the rat central nervous system. Exp. Neurol. 120, 186-201. doi: 10.1006/exnr.1993.1054

Hildebrand, J. G., and Shepherd, G. M. (1997). Mechanisms of olfactory discrimination: converging evidence for common principles across phyla. Annu. Rev. Neurosci. 20, 595-631. doi: 10.1146/annurev.neuro.20.1.595

Hsia, A. Y., Vincent, J. D., and Lledo, P. M. (1999). Dopamine depresses synaptic inputs into the olfactory bulb. J. Neurophysiol. 82, 1082-1085.

Huang, Z., Thiebaud, N., and Fadool, D. A. (2017). Differential serotonergic modulation across the main and accessory olfactory bulbs. J. Physiol. 595, 3515-3533. doi: 10.1113/JP273945

Kapoor, V., Provost, A. C., Agarwal, P., and Murthy, V. N. (2016). Activation of raphe nuclei triggers

rapid and distinct effects on parallel olfactory bulb output channels. Nat. Neurosci. 19, 271-282. doi: $10.1038 / \mathrm{nn} .4219$

Kim, Y. C., Lee, H. G., \& Han, K. A. (2007). D1 dopamine receptor dDA1 is required in the mushroom body neurons for aversive and appetitive learning in Drosophila. Journal of Neuroscience, 27(29), 7640-7647.

Kiyokage, E., Pan, Y. Z., Shao, Z., Kobayashi, K., Szabo, G., Yanagawa, Y., et al. (2010). Molecular identity of periglomerular and short axon cells. J. Neurosci. 30, 1185-1196. doi: 10.1523/JNEUROSCI.3497-09.2010

Kosaka, T., and Kosaka, K. (2016). Neuronal organization of the main olfactory bulb revisited. Anat. Sci. Int. 91, 115-127. doi: 10.1007/s12565-015-0309-7 
Koster, N. L., Norman, A. B., Richtand, N. M., Nickell, W. T., Puche, A. C., Pixley, S. K., et al. (1999). Olfactory receptor neurons express D2 dopamine receptors. J. Comp. Neurol. 411, 666-673. doi: 10.1002/(sici)1096-9861(19990906)411:4<666::aid-cne10>3.0.co;2-s

Langdon, P. E., Harley, C. W., and McLean, J. H. (1997). Increased $\beta$ adrenoceptor activation overcomes conditioned olfactory learning deficits induced by serotonin depletion. Dev. Brain Res. 102, 291293. doi: 10.1016/s0165-3806(97)00090-4

Li, Y., Zhong, W., Wang, D., Feng, Q., Liu, Z., Zhou, J., et al. (2016). Serotonin neurons in the dorsal raphe nucleus encode reward signals. Nat. Coтmun. 7:10503. doi: 10.1038/ncomms 10503

Liu, S., Aungst, J. L., Puche, A. C., and Shipley, M. T. (2012). Serotonin modulates the population activity profile of olfactory bulb external tufted cells. J. Neurophysiol. 107, 473-483. doi: 10.1152/jn.00741.2011

Liu, Z., Zhou, J., Li, Y., Hu, F., Lu, Y., Ma, M., et al. (2014). Dorsal raphe neurons signal reward through

5- $\quad$ HT and glutamate. Neuron 81, 1360-1374. doi: 10.1016/j.neuron.2014.02.010

Lizbinski, K. M., \& Dacks, A. M. (2018). Intrinsic and extrinsic neuromodulation of olfactory processing. Frontiers in cellular neuroscience, 11, 424.

Lucaites, V. L., Krushinski, J. H., Schaus, J. M., Audia, J. E., and Nelson, D. L. (2005). [3H]LY334370, a novel radioligand for the 5- $\mathrm{HT}_{1 \mathrm{~F}}$ receptor. II. Autoradiographic localization in rat, guinea pig, monkey and human brain. Naunyn Schmiedebergs. Arch. Pharmacol. 371, 178-184. doi: 10.1007/s00210005-1036-8

Luo, M., Li, Y., and Zhong, W. (2016). Do dorsal raphe 5-HT neurons encode

"beneficialness"? Neurobiol. ～Learn. Mem. 135, 40-49. doi: 10.1016/j.nlm.2016.08.008

Martin, J. P., Beyerlein, A., Dacks, A. M., Reisenman, C. E., Riffell, J. A., Lei, H., et al. (2011). The neurobiology of insect olfaction: sensory processing in a comparative context. Prog.

Neurobiol. 95, 427-447. doi: 10.1016/j.pneurobio.2011.09.007

McLean, J. H., and Shipley, M. T. (1987). Serotonergic afferents to the rat olfactory bulb: I. Origins and laminar specificity of serotonergic inputs in the adult rat. J. Neurosci. 7, 3016-3028

McLean, J. H., Darby-King, A., Sullivan, R. M., and King, S. R. (1993). Serotonergic influence on olfactory learning in the neonate rat. Behav. Neural Biol. 60, 152-162. doi: 10.1016/01631047(93)90257-i

McLean, J. H., Darby-King, A., and Paterno, G. D. (1995). Localization of 5-HT ${ }_{2 A}$ receptor mRNA by in situ hybridization in the olfactory bulb of the postnatal rat. J. Comp. Neurol. 353, 371-378. doi: $10.1002 /$ cne. 903530305

McLean, J. H., Darby-King, A., and Hodge, E. (1996). 5-HT2 receptor involvement in conditioned olfactory learning in the neonate rat pup. Behav. Neurosci. 110, 1426-1434. doi: 10.1037/07357044.110.6.1426

Miyazaki, K., Miyazaki, K. W., and Doya, K. (2011a). Activation of dorsal raphe serotonin neurons underlies waiting for delayed rewards. J. Neurosci. 31, 469-479. doi:

10.1523/JNEUROSCI.3714- 10.2011

Miyazaki, K. W., Miyazaki, K., and Doya, K. (2011b). Activation of the central serotonergic system in response to delayed but not omitted rewards. Eur. J. Neurosci. 33, 153-160. doi: 10.1111/j.1460-

9568.2010.07480.x 
Muzerelle, A., Scotto-Lomassese, S., Bernard, J. F., Soiza-Reilly, M., and Gaspar, P. (2016). Conditional anterograde tracing reveals distinct targeting of individual serotonin cell groups (B5-B9) to the forebrain and brainstem. Brain Struct. Funct. 221, 535-561. doi: 10.1007/s00429-014-0924-4

Nakamura, K., Matsumoto, M., and Hikosaka, O. (2008). Reward-dependent modulation of neuronal activity in the primate dorsal raphe nucleus. J. Neurosci. 28, 5331-5343. doi: 10.1523/JNEUROSCI.0021-08.2008

Nakamura, K. (2013). The role of the dorsal raphe nucleus in reward-seeking behavior. Front. Integr. Neurosci. 7:60. doi: 10.3389/fnint.2013.00060

Okaty, B. W., Freret, M. E., Rood, B. D., Brust, R. D., Hennessy, M. L., deBairos, D., et al. (2015).

Multi- scale molecular deconstruction of the serotonin neuron system. Neuron 88, 774-791. doi: 10.1016/j.neuron.2015.10.007

Petzold, G. C., Hagiwara, A., and Murthy, V. N. (2009). Serotonergic modulation of odor input to the mammalian olfactory bulb. Nat. Neurosci. 12, 784-791. doi: 10.1038/nn.2335

Pinching, A. J., and Powell, T. P. (1971). The neuropil of the periglomerular region of the olfactory bulb. J. Cell Sci. 9, 379-409.

Pollak Dorocic, I., Fürth, D., Xuan, Y., Johansson, Y., Pozzi, L., Silberberg, G., et al. (2014). A wholebrain atlas of inputs to serotonergic neurons of the dorsal and median raphe nuclei. Neuron 83, 663678. doi: 10.1016/j.neuron.2014.07.002

Price, T. L., Darby-King, A., Harley, C. W., and McLean, J. H. (1998). Serotonin plays a permissive role in conditioned olfactory learning induced by norepinephrine in the neonate rat. Behav. Neurosci. 112, 1430-1437. doi: 10.1037/0735-7044.112.6.1430

Ranade, S. P., and Mainen, Z. F. (2009). Transient firing of dorsal raphe neurons encodes diverse and specific sensory, motor, and reward events. J. Neurophysiol. 102, 3026-3037. doi: 10.1152/jn.00507.2009

Ren, J., Friedmann, D., Xiong, J., Liu, C. D., Ferguson, B. R., Weerakkody, T., ... \& Weissbourd, B. (2018). Anatomically Defined and Functionally Distinct Dorsal Raphe Serotonin Sub-systems. Cell.

Riffell, J. A., Lei, H., Abrell, L., \& Hildebrand, J. G. (2013). Neural basis of a pollinator's buffet: olfactory specialization and learning in Manduca sexta. Science, 339(6116), 200-204.

Saraswati, S., Adolfsen, B., \& Littleton, J. T. (2007). Characterization of the role of the Synaptotagmin family as calcium sensors in facilitation and asynchronous neurotransmitter release. Proceedings of the National Academy of Sciences, 104(35), 14122-14127.

Schendzielorz, T., Schirmer, K., Stolte, P., \& Stengl, M. (2015). Octopamine regulates antennal sensory neurons via daytime-dependent changes in cAMP and IP3 levels in the hawkmoth Manduca sexta. PLoS One, 10(3), e0121230.

Schmidt, L. J., and Strowbridge, B. W. (2014). Modulation of olfactory bulb network activity by serotonin: $\quad$ synchronous inhibition of mitral cells mediated by spatially localized GABAergic microcircuits. Learn. Mem. 21, 406-416. doi: 10.1101/lm.035659.114

Schroll, C., Riemensperger, T., Bucher, D., Ehmer, J., Völler, T., Erbguth, K., ... \& Fiala, A. (2006). Light- induced activation of distinct modulatory neurons triggers appetitive or aversive learning in Drosophila larvae. Current biology, 16(17), 1741-1747. 
Schwaerzel, M., Monastirioti, M., Scholz, H., Friggi-Grelin, F., Birman, S., \& Heisenberg, M. (2003). Dopamine and octopamine differentiate between aversive and appetitive olfactory memories in Drosophila. Journal of Neuroscience, 23(33), 10495-10502.

Seroogy, K. B., Brecha, N., and Gall, C. (1985). Distribution of cholecystokinin-like immunoreactivity in the rat main olfactory bulb. J. Comp. Neurol. 239, 373-383. doi: 10.1002/cne.902390403

Shen, Y., Monsma, F. J. Jr., Metcalf, M. A., Jose, P. A., Hamblin, M. W., and Sibley, D. R. (1993).

Molecular cloning and expression of a 5-hydroxytryptamine7 serotonin receptor subtype. J. Biol. Chem. 268, 18200-18204.

Shipley, M. T., and Ennis, M. (1996). Functional organization of olfactory system. J. Neurobiol. 30, 123176. doi: 10.1002/(sici)1097-4695(199605)30:1<123::aid-neu11>3.3.co;2-s

Sizemore, T. R., \& Dacks, A. M. (2016). Serotonergic modulation differentially targets distinct network elements within the antennal lobe of Drosophila melanogaster. Scientific reports, 6, 37119.

Steinfeld, R., Herb, J. T., Sprengel, R., Schaefer, A. T., and Fukunaga, I. (2015). Divergent innervation of the olfactory bulb by distinct raphe nuclei. J. Comp. Neurol. 523, 805-813. doi: $10.1002 /$ cne. 23713

Suzuki, Y., Kiyokage, E., Sohn, J., Hioki, H., and Toida, K. (2015). Structural basis for serotonergic regulation of neural circuits in the mouse olfactory bulb. J. Comp. Neurol. 523, 262-280. doi: $10.1002 / \mathrm{cne} .23680$

Tecott, L. H., Maricq, A. V., and Julius, D. (1993). Nervous system distribution of the serotonin 5$\mathrm{HT}_{3}$ receptor mRNA. Proc. Natl. Acad. Sci. U S A 90, 1430-1434. doi: 10.1073/pnas.90.4.1430

Unoki, S., Matsumoto, Y., \& Mizunami, M. (2005). Participation of octopaminergic reward system and dopaminergic punishment system in insect olfactory learning revealed by pharmacological study. European journal of Neuroscience, 22(6), 1409-1416.

Vinauger, C., Lahondère, C., Wolff, G. H., Locke, L. T., Liaw, J. E., Parrish, J. Z., ... \& Riffell, J. A. (2018). Modulation of host learning in Aedes aegypti mosquitoes. Current Biology, 28(3), 333-344.

Waeber, C., Grailhe, R., Yu, X. J., Hen, R., and Moskowitz, M. A. (1998). Putative 5-ht 5 receptors: localization in the mouse CNS and lack of effect in the inhibition of dural protein extravasation. Ann. N Y Acad. Sci. 861, 85-90. doi: 10.1111/j.1749-6632.1998.tb10177.x

Watts, S. W., Gackenheimer, S. L., Gehlert, D. R., and Cohen, M. L. (1994). Autoradiographic comparison of [125I]LSD-labeled 5- $\mathrm{HT}_{2 \mathrm{~A}}$ receptor distribution in rat and guinea pig brain. Neurochem. Int. 24, 565-574. doi: 10.1016/0197-0186(94)90009-4

Weissbourd, B., Ren, J., DeLoach, K. E., Guenthner, C. J., Miyamichi, K., and Luo, L. (2014). Presynaptic partners of dorsal raphe serotonergic and GABAergic neurons. Neuron 83, 645-662. doi: 10.1016/j.neuron.2014.06.024

Wen, H., Linhoff, M. W., McGinley, M. J., Li, G. L., Corson, G. M., Mandel, G., \& Brehm, P. (2010). Distinct roles for two synaptotagmin isoforms in synchronous and asynchronous transmitter release at zebrafish neuromuscular junction. Proceedings of the National Academy of Sciences, 107(31), 13906-13911.

Wilson, R. I. (2013). Early olfactory processing in Drosophila: mechanisms and principles. Annu. Rev. Neurosci. 36, 217-241. doi: 10.1146/annurev-neuro-062111-150533 
Wright, D. E., Seroogy, K. B., Lundgren, K. H., Davis, B. M., and Jennes, L. (1995). Comparative localization of serotonin ${ }_{1 \mathrm{~A}}, 1 \mathrm{C}$, and ${ }_{2}$ receptor subtype mRNAs in rat brain. J. Comp. Neurol. 351, 357-373. doi: 10.1002/cne.903510304

Yuan, Q., Harley, C. W., and McLean, J. H. (2003). Mitral cell $\beta 1$ and 5-HT $2 \mathrm{~A}$ receptor colocalization and cAMP coregulation: a new model of norepinephrine-induced learning in the olfactory bulb. Learn. Mem. 10, 5-15. doi: 10.1101/lm.54803

Zhang, X., \& Gaudry, Q. (2016). Functional integration of a serotonergic neuron in the Drosophila antennal lobe. Elife, 5, e16836.

Zheng, Z., Lauritzen, J. S., Perlman, E., Robinson, C. G., Nichols, M., Milkie, D., ... \& Calle-Schuler, S. A. (2018). A complete electron microscopy volume of the brain of adult Drosophila melanogaster. Cell, 174(3), 730-743.

Zhong, W., Li, Y., Feng, Q., and Luo, M. (2017). Learning and stress shape the reward response patterns of serotonin neurons. J. Neurosci. 37, 8863-8875. doi: 10.1523/JNEUROSCI.1181-17.2017 